\title{
Transcription regulation dynamics
}

Wim J. de Jonge 
ISBN

Copyright

Cover art and illustrations

Layout

Printed by
978-90-393-7305-7

(c) 2020 W. J. de Jonge, all rights reserved

Geerte van Beers I geertevanbeers@gmail.com Geerte van Beers \& W. J. de Jonge

Ridderprint I www.ridderprint.nl 


\title{
Transcription regulation dynamics
}

\author{
Dynamiek van transcriptie regulatie
}

(met een samenvatting in het Nederlands)

\section{Proefschrift}

ter verkrijging van de graad van doctor aan de Universiteit Utrecht op gezag van de rector magnificus, prof.dr. H.R.B.M. Kummeling, ingevolge het besluit van het college voor promoties in het openbaar te verdedigen op

donderdag 3 september 2020 des middags te 2.30 uur

door

Willem Jozef de Jonge

geboren op 20 augustus 1990

te 's-Hertogenbosch 
Promotor:

Prof. dr. F.C.P. Holstege

Copromotor:

Dr. P.P.C.W. Kemmeren 
The distance between insanity and genius is measured only by success.

Bruce Feirstein 



\section{Table of contents}

$\begin{array}{lll}\text { Chapter } 1 \quad \text { General Introduction } & 9\end{array}$

Chapter 2 Molecular mechanisms that distinguish TFIID 41 housekeeping from regulatable SAGA promoters

75

Chapter 3 Extensive optimization of chromatin immunoprecipitation for quantitatively comparable and robust results

Chapter 4 An optimized chromatin immunoprecipitation protocol for quantification of protein-DNA interactions

Chapter 5 Genome-wide off-rates reveal how DNA binding dynamics shape transcription factor function

Chapter 6 General Discussion

$\begin{array}{lll}\text { Addendum Nederlandse Samenvatting } & 216\end{array}$

$\begin{array}{ll}\text { Author contributions per chapter } & 220\end{array}$

$\begin{array}{ll}\text { List of publications } & 221\end{array}$

$\begin{array}{ll}\text { Curriculum Vitae } & 222\end{array}$

Acknowledgements 223 



\section{Chapter 1}

\section{General Introduction}

Wim J. de Jonge, Patrick Kemmeren and Frank C.P. Holstege

Princess Máxima Center for Pediatric Oncology, Heidelberglaan 25, 3584 CS Utrecht, the Netherlands

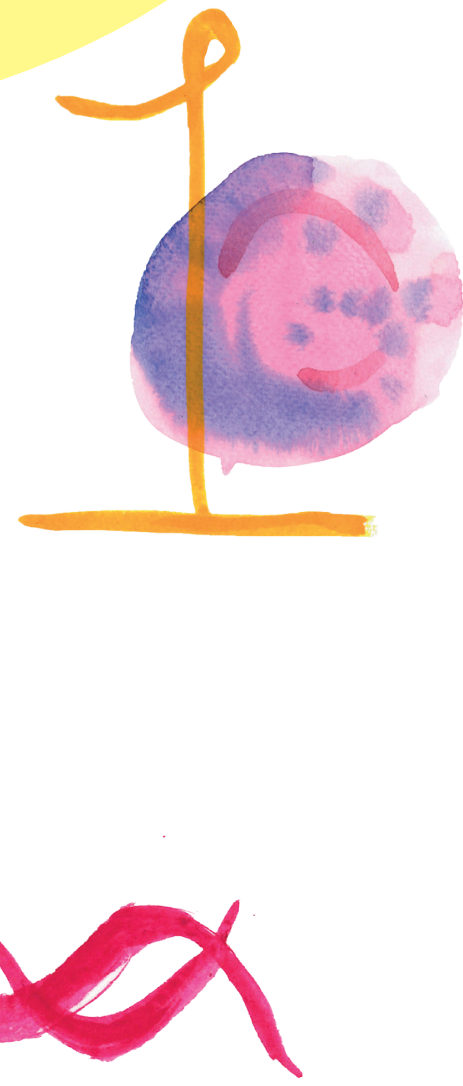




\section{DNA and transcription}

DNA is fundamental to all life and contains the building instructions for different cell types as well as information required for cells to perform their various functions. In eukaryotes, DNA is contained in the nucleus in the form of a higher-order structure called chromatin. Chromatin consists of DNA and DNA-bound proteins. The main component of chromatin is the nucleosome, which consists of DNA wrapped around an octamer of histone proteins. Nucleosomes are needed to compact the negatively charged DNA to fit in the nucleus, but they are also important for regulating DNAbased processes. One of these processes is transcription. During transcription, the information of a DNA strand is copied into a strand of RNA, for example messenger RNA (mRNA). mRNA can be translated by ribosomes into proteins, which carry out the majority of the functions in the cell. Besides mRNA, there are several other types of RNAs known. Ribosomal (r)RNAs, which are a structural part of the ribosomes, transfer $(\mathrm{t}) \mathrm{RNAs}$ that are needed to translate mRNA into proteins and many different types of non-coding (nc)RNAs that can have roles in regulating various processes in the cell (Lee \& Young, 2013). Different types of RNAs are transcribed by different RNA polymerases. RNA polymerase I transcribes the large precursor rRNA, RNA polymerase II is responsible for transcribing mRNAs and various ncRNAs and RNA polymerase III transcribes tRNAs and the small rRNA (Cramer, 2019). Plants have a fourth polymerase, RNA polymerase IV, which transcribes specific ncRNAs (Onodera et al, 2005).

The specific proteins that are present in a cell determine for a large part the cell's identity and function, and which proteins are present in a cell largely depends on which parts of the genome are transcribed. Transcription is therefore arguably one of the most important processes that defines differences between cell types from the same organism and how they interact with their environment. It is therefore important that DNA is transcribed at the right place and the right time. For example, a human hepatocyte synthesizes alcohol dehydrogenase, while there is little use for this protein in other cells such as keratinocytes or fibroblasts. Also singlecelled organisms such as yeast need to transcribe different parts of their genome at different times, depending on input from external signals such as the presence of carbon sources or elevated temperatures (Gasch et al, 2000; López-Maury et al, 2008). Therefore, transcriptional programs must constantly change and transcription is thus a dynamic process. During development in multicellular organisms, transcriptional programs change over the course of hours or days (Yi et al, 2010; Takaoka \& Hamada, 2012; White et al, 2017). Responses to changing environments need to be rapid and a cell needs to adapt to different circumstances within minutes. Thus, which genes are expressed at a particular moment in a particular cell is constantly changing. Since transcription itself is dynamic, the factors that regulate this process also have to be dynamic, probably on an even faster timescale.

The work in this thesis focuses on the dynamics of RNA polymerase II transcription regulation in the yeast Saccharomyces cerevisiae. Many proteins involved in transcription are highly conserved between eukaryotes. This means that on a fundamental level transcription is similar between yeast and more complex 
eukaryotes such as humans. Compared to humans, yeast has a roughly 250x smaller genome and about one third of the number of genes. The genome is therefore more compact and regulation happens over smaller distances. This, together with the long history of studying transcription in yeast and the availability of many high-quality datasets (reviewed in Botstein \& Fink, 2011) makes yeast an excellent model system to study molecular mechanisms of transcription regulation and its dynamics.

\section{The transcription cycle}

Transcription itself consists of many steps each of which can be regulated (Margaritis \& Holstege, 2008; Fuda et al, 2009). The first step usually consists of binding of proteins called transcription factors (TFs) to DNA by recognizing specific DNA motifs. These motifs are present in the regulatory sequence upstream of a gene, called the promoter. If the TF is an activating $\mathrm{TF}$, binding will lead to recruitment, usually indirectly, of the pre-initiation complex (PIC). The PIC consists of RNA polymerase II (Pol II) and several other proteins and protein complexes that facilitate the initiation of transcription. Upon separation of the two DNA strands, transcription can initiate, Pol II goes into the elongation phase and transcribes the DNA into RNA. During elongation, Pol II is phosphorylated at different residues in the C-terminal domain (CTD) of its largest subunit. This CTD consists of many repeats of seven amino acids, out of which five can be phosphorylated. The pattern of phosphorylation changes during elongation. Different combinations of phosphorylated residues allow for recruitment of different factors that are needed during elongation. Through changes in the pattern of phosphorylated residues, the appropriate factors can be dynamically recruited and released at the right time. Pol II will continue to elongate until small sequences are incorporated into the RNA. These sequences, together with specific modifications of the CTD, are recognized by termination factors that will cause Pol II to terminate (Porrua \& Libri, 2015). When polymerase terminates, the RNA is released, processed and in the case of mRNA transported to the cytoplasm to be translated into proteins. The whole process of transcription is dynamically regulated by various factors and mis-regulation at any of the steps can have severe consequences for the cell (Lee \& Young, 2013). Despite regulation taking place at all steps, recruitment of the PIC is often regarded as one of the most highly regulated steps in transcription. Spatiotemporal control of PIC recruitment to specific genes is largely regulated by TFs.

\section{Gene specific transcription factors dynamically regulate their targets}

Which genes are activated or repressed at a given moment is largely determined by gene-specific transcription factors (GSTFs). To achieve spatiotemporal control, GSTFs have to bind and release from DNA in a dynamic manner. GSTFs bind to specific promoters by recognizing small motifs in the DNA. Besides the motif sequence itself, characteristics of the DNA shape, such as width of the minor groove or the rotation angle between bases at the binding motif location, can also contribute to GSTF binding site recognition (Rohs et al, 2009; Zhou et al, 2013). Multiple GSTFs may bind the promoter of the same gene, allowing for integration of external signals and this is referred to as combinatorial control (Reményi et al, 2004; Hahn \& Young, 2011; Brkljacic \& Grotewold, 2017). This is an important concept because it allows a 
small set of GSTF to control a large set of genes in response to various environmental changes.

Most of the highly studied GSTFs, especially in yeast, are the ones that regulate responses to environmental signals. For example, the GSTF Gal4 regulates genes that are needed when galactose is used as a carbon source and Pho4 regulates the cells' response to the availability of phosphate (Giniger et al, 1985; Lenburg \& O'Shea, 1996). Responses to suboptimal growth conditions, often referred to as stress, such as elevated temperatures or high concentration of salt are mediated by GSTFs as well. To allow for rapid responses, GSTFs that control these responses must bind dynamically to their target promoters. For example, the GSTF Msn2 regulates responses to stress, such as oxidative or salt stress (Hao \& O'Shea, 2012). In non-stress conditions, Msn2 is phosphorylated and exported from the nucleus (Görner et al, 1998). When the cells are stressed, the kinase responsible for Msn2 phosphorylation is inhibited and Msn2 is translocated to the nucleus. The dynamics of Msn2 re-localization, i.e. the amplitude, frequency and duration, differs depending on the source of the stress (Hao \& O'Shea, 2012). Different target genes are activated with different kinetics, depending on the dynamics of the re-localization (Hansen \& O'Shea, 2013). For example, some genes are activated within minutes after Msn2 is re-localized to the nucleus, while others only become active upon prolonged Msn2 nuclear localization. This shows that genes can be differentially regulated through different dynamics of a single TF. Another example of the importance of binding dynamics in response to a changing environment comes from the Drosophila GSTF HSF (heat shock factor), which is homologous to the yeast GSTF Hsf1. As the name suggests, HSF mediates the response to heat stress. HSF is located in the nucleus under standard conditions, but only binds transiently to DNA. However, after cells receive a heat shock, HSF becomes much more stably bound, with a half-life of at least several minutes (Yao et al, 2006). This allows its targets to become highly expressed. Besides environmental signals, other proteins in the cell such as chaperones or the proteasome can also control dynamics of TF binding (Elbi et al, 2004; Stavreva et al, 2004; Bosisio et al, 2006). Together, this highlights that transcriptional responses to changing environments, regulation of gene expression and binding of regulators are inherently dynamic and important factors that are actively controlled.

Besides GSTFs that often regulate more specific responses to environmental signals, there is a class of TFs that has a broader role in regulating transcription in yeast. These factors are therefore called general regulatory factors (GRFs). There are six members of this class: Abf1, Cbf1, Mcm1, Rap1, Reb1 and Tbf1. GRFs are highly abundant TFs that can contribute to nucleosome positioning (Kent et al, 2004; Hartley \& Madhani, 2009; Ganapathi et al, 2011) and are therefore akin to pioneering TFs in more complex eukaryotes (Zaret \& Carroll, 2011; Kubik et al, 2017b). All GRFs but Cbf1 are essential for cell viability (Passmore et al, 1988; Rhode et al, 1989; Cai \& Davis, 1990; Ju et al, 1990; Brigati et al, 1993), which highlights the importance of GRFs for normal cell function. GRFs control the transcription of genes involved in various processes. For example, the majority of genes encoding for ribosomal subunits are bound and regulated by Rap1 (Knight et al, 2014), while a small subset is under the control of Abf1 (Lascaris 
et al, 1999; Fermi et al, 2016). Ribosomal proteins are abundant and highly expressed. In contrast to other promoters where Rap1 binds more dynamically, Rap1 binding to the ribosomal genes is very stable (Lickwar et al, 2012). This likely facilitates high levels of Pol II recruitment and therefore high expression levels of ribosomal protein genes. Mcm1 controls expression of genes involved in mating, but also in arginine biosynthesis, cell wall maintenance and cell cycle progression (Kuo \& Grayhack, 1994; Messenguy \& Dubois, 2003). Interestingly, Mcm1 activates or represses several mating-type specific genes based on the mating type of the cell (reviewed in Haber, 2012). Cb1f has been shown to be able to both activate and repress transcription as well (Kemmeren et al, 2014). GRFs can also regulate transcription by fine-tuning termination. Multiple GRFs were shown to limit pervasive transcription by acting as a roadblock for transcribing Pol II, causing Pol II to terminate (Colin et al, 2014; Roy et al, 2016; Candelli et al, 2018). Thus, GRFs can regulate transcription in various ways.

Besides functioning in transcriptional control, GRFs are also important for various other processes. Abf1 is involved in DNA replication and DNA repair (Marahrens \& Stillman, 1992; Reed et al, 1999) and Cbf1 is important for proper chromosome segregation (Cai \& Davis, 1990). Abf1, Rap1 and Tbf1 also bind to telomeres and Tbf1 and Rap1 are important for telomere maintenance (Lustig et al, 1990; Brigati et al, 1993; Enomoto et al, 1994; Pryde \& Louis, 1999; Fukunaga et al, 2011). Since GRFs are involved in different processes, that all have different dynamics, the binding of GRFs to sites involved in these processes likely also have different dynamics. Indeed, Rap1 was shown to bind less stably to telomeres compared to promoters (Lickwar et al, 2012).

In conclusion, there are different classes of TFs that regulate transcription. These TFs likely have different dynamics based on the context where they are bound. Yet, very little is known about what determines differential binding dynamics between sites, and how these dynamics relate to their different functions and modes of action.

\section{General transcription factors and transcription initiation}

The main role for activating transcription factors is recruiting the PIC. Besides Pol II, the PIC consists of several general transcription factors (GTFs), named because of their general requirement for Pol II transcription. The classical view of PIC formation is that of a step-wise assembly (reviewed in Sainsbury et al, 2015), although other assembly pathways are possible as well. The first step of canonical PIC formation is binding of TBP to a stretch of DNA called the TATA-box (Sainsbury et al, 2015). The TATA-box has the sequence TATAWAWR (with $W$ indicating A or T and $R$ a purine). Although most of the early work studying transcription regulation was performed on promoters that contain a canonical TATA-box, the majority of the genes have a TATA-like sequence with one or two mismatches (Basehoar et al, 2004; Rhee \& Pugh, 2012). TBP can be brought to the promoter by TFIID, but TFIID in its entirety is not strictly required for transcription of all genes in vitro or in vivo (Hahn et al, 1989; Horikoshi et al, 1989; Kuras et al, 2000; Lee et al, 2000; Huisinga \& Pugh, 2004; Petrenko et al, 2019; Donczew et al, 2020). TFIIA can stabilize the binding of TBP to 
DNA in vitro, but is dispensable for binding (Imbalzano et al, 1994). This fits with studies showing that TFIIA has a stimulatory role, but is not strictly required for transcription in vivo (Liu et al, 1999; Stargell et al, 2000). More recent experiments however, suggest that all transcription is dependent on TFIIA (Petrenko et al, 2019). Besides TFIIA, TFIIB also stabilizes TBP binding (Imbalzano et al, 1994). TFIIB is required to recruit Pol II to the promoter (Buratowski et al, 1989) and for proper start site selection (Li et al, 1994). TFIIB recruits Pol II and TFIIF, and TFIIB binding to the PIC in turn is stabilized by TFIIF (Čabart et al, 2011). Approximately 50\% of Pol II in vivo is bound by TFIIF (Rani et al, 2004) and TFIIF prevents aspecific binding of Pol II to DNA (Conaway et al, 1991). At this stage the DNA is still double stranded, and the two strands need to be separated to allow transcription to start. The DNA is opened by TFIIH (Holstege et al, 1996) and TFIIE is needed to recruit TFIIH by acting as a bridge between TFIIH and Pol II (Maxon et al, 1994). When the DNA is opened, a phosphodiester bond can be formed between DNA and the first RNA base. After several rounds of abortive initiation, a stable transcript can be formed and Pol II transitions to the elongation phase (Margaritis \& Holstege, 2008).

\section{Co-activators}

GSTFs generally do not recruit the PIC directly, but recruit co-activators that promote the assembly of the PIC. These co-activators are large mega Dalton complexes that consist of more than 10 subunits each, and are conserved between eukaryotes (Hahn \& Young, 2011). Two co-activators, TFIID and SAGA, can bring TBP to the promoter. Although most genes rely mainly on TFIID to bring TBP to the promoter, a subset of approximately $15 \%$ of the genes also rely on SAGA (Lee et al, 2000; Huisinga \& Pugh, 2004; Rhee \& Pugh, 2012; Donczew et al, 2020). Mediator is another co-activator that was shown to be generally required for transcription (Thompson \& Young, 1995; Holstege et al, 1998). Mediator can bind to activating transcription factors as well as Pol II and promotes recruitment of the PIC (reviewed in Kornberg, 2005; and Conaway \& Conaway, 2011). Phosphorylation of the CTD of Pol II by TFIIH disrupts Mediator binding and allows Pol II escape from the promoter (Jeronimo \& Robert, 2014; Wong et al, 2014).

SAGA and TFIID both bring TBP to the promoter, but the dynamics of TBP binding is different depending on whether it binds in the context of SAGA or TFIID. TBP bound sites with high turnover are enriched for dependence on SAGA and conversely sites with low turnover are enriched for dependence on TFIID (van Werven et al, 2009). A factor that may cause these differences in turnover is Mot1, which can actively dissociate TBP from the promoter and thus increase turnover (Auble et al, 1994; van Werven et al, 2008). Based on the crystal structures of TBP, Mot1 and TFIID, Mot1 cannot compete with TFIID for binding to TBP, which could explain why genes dependent on SAGA are more sensitive to destabilization of TBP by Mot1 (Ravarani et al, 2016). Genome-wide expression analysis showed that upon depletion of Mot1 mainly genes dependent on SAGA are upregulated, which is consistent with Mot1 removing TBP specifically from these promoters (Spedale $e t$ al, 2012). These results emphasize that besides GSTFs, components of the PIC and in particular TBP dynamically bind DNA as well, and that co-activator usage likely 
plays a role in this process.

\section{Nucleosome architecture at promoters and nucleosome dynamics}

To gain access to DNA, GSTFs and coactivators need to compete with nucleosomes. Besides DNA, a nucleosome consists of two dimers of histone $\mathrm{H} 3$ and $\mathrm{H} 4$ and two dimers of histone H2A and H2B (Luger et al, 1997). Occasionally, different histone variants such as histone variant $\mathrm{H} 2 \mathrm{~A}$. Z may be incorporated, which is important for regulating transcription (Santisteban et al, 2000). Nucleosomes have a stereotypical architecture at the promoter (Yuan et al, 2005; Lee et al, 2007; Jiang \& Pugh, 2009). Most genes have a nucleosome that covers the transcription start site (TSS), and this nucleosome is referred to as the " +1 nucleosome" (Fig1A). The +1 nucleosome of a gene is often well-positioned, meaning that there is little variation in the position where it is bound between different cells in a population of yeast under the same growth condition. The region just upstream of the +1 nucleosome is often relatively depleted of nucleosomes. This region is therefore referred to as the NFR (nucleosome free region) or NDR (nucleosome depleted region). NFRs are enriched for homopolymeric stretches of deoxyadenosine or deoxythymine, also called poly(dA:dT) motifs (Yuan et al, 2005; Lee et al, 2007; Mavrich et al, 2008). These poly(dA:dT) motifs are inherently stiff, which resists wrapping around histones. The presence of poly(dA:dT) motifs may therefore contribute to NFR formation (Struhl, 1985; Iyer \& Struhl, 1995; Kaplan et al, 2009; Segal \& Widom, 2009). The NFR is important for transcription regulation as TF binding sites are often located in the NFR (Yuan et al, 2005). The nucleosome that is positioned upstream of the NFR is referred to as the "-1 nucleosome" (Fig 1A). When examining the average nucleosome positioning between all genes in the genome, the +1 nucleosome is the most uniformly positioned nucleosome between genes. The downstream nucleosomes are positioned with $\sim 18 \mathrm{bp}$ spacing, or linkers, between the nucleosomes. On average, positioning becomes less uniform between genes for nucleosomes more downstream of the TSS.

This traditional view of nucleosome positioning comes mainly from experiments where chromatin is digested using micrococcal nuclease (MNase), which digests accessible DNA. DNA that is bound by nucleosomes is protected and can be detected

A

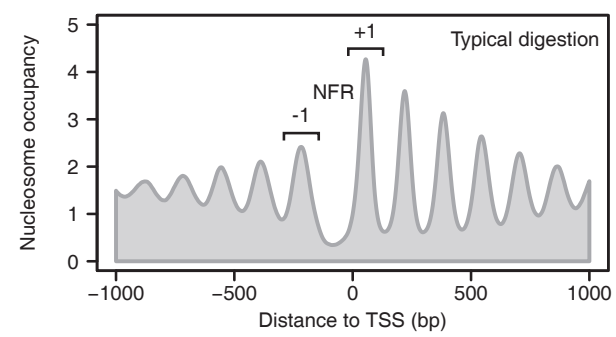

B

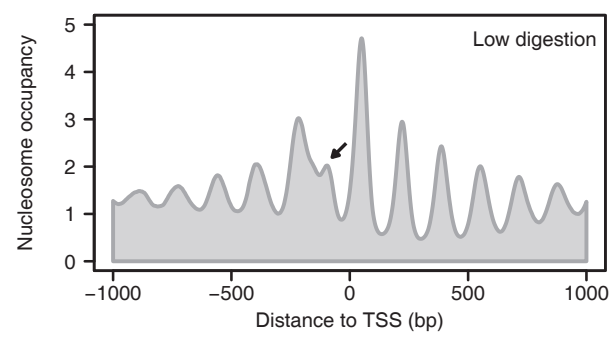

Figure 1. Average nucleosome organization at yeast genes

(A) Average nucleosome occupancy measured using MNase-seq at 5,043 yeast genes aligned to their transcription start site. The NFR and flanking -1 and +1 nucleosomes are indicated. (B) Average nucleosome occupancy as in (A) but treated using a lower concentration of MNase. The "fragile" nucleosome present in NFRs is indicated with an arrow. Note the relative difference in occupancy of the +1 and downstream nucleosomes between the two digestions. 
by sequencing after MNAse digestion (MNase-seq). Typically the chromatin would be treated such that most of the linkers between nucleosomes are digested and only DNA fragments that are bound by a single nucleosome remain (Rando, 2010). However, not all nucleosomes protect DNA to the same degree. Treating samples with lower amounts of MNase led to the discovery of so called "fragile nucleosomes" (Fig 1B, arrow), which are nucleosomes that are particularly sensitive to MNase treatment and are mainly located in NFRs (Weiner et al, 2010; Xi et al, 2011; Kubik et al, 2015). These fragile nucleosomes appear when NFRs are big enough to accommodate a nucleosome. There has been debate in the literature whether these fragile nucleosomes are really nucleosomes or other protein complexes that offer protection against MNase (Chereji et al, 2017; Kubik et al, 2017a). They likely represent nucleosomes that are partially unwrapped due to the binding of other proteins (Brahma \& Henikoff, 2019). Still, fragile nucleosomes are not the only nucleosomes that are differentially affected by different concentrations of MNase. Therefore, the average nucleosome architecture differs depending on how heavily the sample was treated (compare Fig 1A and 1B). This shows that to get a full picture of nucleosome positioning using MNase-seq, it is important to analyze both more heavily and more lightly treated samples.

Even though nucleosomes tightly bind DNA, nucleosomes are dynamic structures (reviewed in Lai \& Pugh, 2017). For example, during DNA replication a cell has to double its DNA content and to allow DNA polymerase to copy the DNA it needs to be free of nucleosomes (Ransom et al, 2010). This means that at least once during the cell cycle, which lasts about 90 minutes for yeast growing with glucose as a carbon source, all nucleosomes dissociate and rebind DNA. Nucleosomes are also dynamic outside of the cell cycle, depending on their location relative to genes. Nucleosomes with high turnover are usually found in the promoter or close to the TSS, while nucleosomes with low turnover are found in the coding region (Dion et al, 2007; Rufiange et al, 2007). Promoter nucleosomes, in particular the +1 nucleosome, are enriched for the histone variant H2A.Z (Albert et al, 2007). The +1 nucleosome forms a barrier for the progression of Pol II, but incorporation of H2A.Z makes the +1 nucleosome a weaker barrier (Weber et al, 2014). The presence of H2A.Z correlates with turnover (Dion et al, 2007) and yeast nucleosomes containing H2A.Z are less stable in vitro. This suggests that lower stability may facilitate nucleosome loss at promoters (Zhang et al, 2005). Thus, both the binding and the composition of nucleosomes are dynamic, and important for regulating transcription.

Nucleosome positioning is important for gene regulation, in particular the +1 nucleosome that covers the TSS, since the PIC has to be assembled close to this nucleosome (Rhee \& Pugh, 2012). The position of the +1 nucleosome relative to the TSS was shown to be important for regulation of the highly expressed ribosomal protein genes (Reja et al, 2015) as well as metabolically cycling genes (Nocetti \& Whitehouse, 2016). The exact position of the +1 nucleosome is highly regulated to allow access of TBP to the TATA-box (Kubik et al, 2018). In addition, the position of the +1 nucleosome is important for proper start site selection and prevents ectopic initiation (Challal et al, 2018), likely by suppressing initiation driven by cryptic 
TATA-elements (Kubik et al, 2019).

Nucleosomes themselves are dynamic, but can also contribute to the dynamics of TFs. For example, the TF Gal4 stably binds naked DNA in vitro, but upon addition of nucleosomes the time Gal4 stays bound to DNA (residence time) is reduced more than a 1000-fold (Luo et al, 2014). Similarly, the presence of nucleosomes also reduces the residence time of the GRF Rap1, albeit to a lesser degree (Mivelaz et al, 2020). This provides an explanation as to why TF binding has been observed to be more dynamic in vivo than in vitro. Strikingly, for two other GRFs, Reb1 and Cbf1, binding to nucleosomal DNA increases residence time (Donovan et al, 2019a). These studies show that nucleosome binding can have strong and distinct effects on TF binding stability, depending on the TF and the position of the nucleosome relative to the TF binding motif. This highlights the importance of studying transcription factor binding dynamics in vivo. Nucleosomes restrict access to DNA for the majority of TFs (Zhu et al, 2018), indicating that nucleosomes are important modulators of TF binding and dynamics.

\section{Chromatin remodelers regulate nucleosome positioning and dynamics}

Dynamic positioning of nucleosomes is largely determined by chromatin remodelers, which hydrolyze ATP to reposition or evict nucleosomes. Various chromatin remodelers are present in yeast and all are conserved between eukaryotes (Clapier \& Cairns, 2009). Chromatin remodelers are important modulators of gene expression and individual deletions of the majority of remodeler subunits has clear effects on transcription (Lenstra et al, 2011). Chromatin remodelers bind and reposition different nucleosomes (Yen et al, 2012) and control nucleosome positioning mainly in three different ways. First, by moving nucleosomes away from the NFR and thereby increasing NFR size. Second, by moving nucleosomes towards the NFR and thus decreasing NFR size and last by affecting spacing between nucleosomes across gene bodies (Yen et al, 2012; Krietenstein et al, 2016; Kubik et al, 2019).

Chromatin remodelers that increase NFR size are SWI/SNF and RSC (Hartley \& Madhani, 2009; Tolkunov et al, 2011; Krietenstein et al, 2016; Kubik et al, 2018, 2019). These complexes are homologs and both are large complexes that can engulf a nucleosome (Chaban et al, 2008; Dechassa et al, 2008; Han et al, 2020; Wagner et al, 2020). The RSC complex is the only chromatin remodeler that is essential for yeast viability (Cairns et al, 1996) and upon depletion of RSC from the nucleus, the majority of NFRs shrink (Hartley \& Madhani, 2009; Kubik et al, 2018, 2019). RSC binds to DNA by recognizing GC-rich motifs through its Rsc3 and Rsc30 subunits (Badis et al, 2008). The activity of RSC is stimulated by the presence of poly(dA:dT) motifs (Lorch et al, 2014; Kubik et al, 2015). The proximity, orientation and location of the GC-rich and poly(dA:dT) motifs modulates RSC binding and activity (Krietenstein et al, 2016; Kubik et al, 2018). SWI/SNF regulates nucleosome positioning and NFR size of a smaller subset of promoters (Tolkunov et al, 2011; Kubik et al, 2019) and SWI/SNF can be targeted to promoters by GSTFs (Neely et al, 1999; Yudkovsky et al, 1999). 
The effects of chromatin remodelers that increase NFR size are counteracted by remodelers that decrease NFR size: INO80, ISW1a and ISW2 (Whitehouse et al, 2007; Yen et al, 2012; Krietenstein et al, 2016; Kubik et al, 2019). In vitro data showed that for a subset of promoters INO80 is able to position the +1 nucleosome on its own, likely by reading out DNA shape features at these positions (Krietenstein et al, 2016). Deletion or depletion of the catalytic subunit Ino80 in vivo shifts nucleosomes away from the NFR, showing that INO80 repositions nucleosomes towards the NFR in vivo (Yen et al, 2012; Kubik et al, 2019). INO80 can also remove histone H2A.Z from the +1 nucleosome, thereby regulating H2A.Z binding dynamics (Papamichos-Chronakis et al, 2011). The INO80 driven exchange of H2A.Z contributes to turnover of the +1 nucleosome (Yen et al, 2013). In yeast there are two ISW1 complexes, ISW1a and ISW1b, that share the same catalytic subunit, Isw1 (Vary et al, 2003). In vivo deletion or depletion of Isw1 results mainly in more irregular spacing of nucleosomes in gene bodies (Tirosh et al, 2010; Gkikopoulos et al, 2011; Yen et al, 2012; Kubik et al, 2019). Interestingly, ISW1a binds to the +1 nucleosome (Yen et al, 2012) and in vitro data indicate that ISW1a can position the +1 nucleosome by using Abf1 as a barrier (Krietenstein et al, 2016). ISW2 can also position the +1 nucleosome together with a GRF barrier in vitro (Krietenstein et al, 2016). Deletion or depletion of Isw2 in vivo shifts nucleosomes away from the NFR, similarly as INO80, indicating that ISW2 positions nucleosomes towards the NFR in vivo (Whitehouse et al, 2007; Yen et al, 2012; Kubik et al, 2019). Thus, the +1 nucleosome does not occupy a fixed position in vivo, but its exact position is highly dynamic and is regulated by various chromatin remodelers.

Lastly, remodelers also determine spacing between nucleosomes that are downstream of the +1 nucleosome and cover the gene body. Besides the aforementioned ISW1 remodelers, CHD1 also plays an important role in nucleosome spacing. Deletion of both ISW1 and CHD1 leads to a severe spacing defect in vivo (Gkikopoulos et al, 2011). Besides the ISW1 and CHD1 complexes, ISW2 and INO80 also can contribute to nucleosome spacing, albeit to a lesser degree (Udugama et al, 2011; Ocampo et al, 2016). All these factors appear to act highly redundantly for spacing nucleosomal arrays, and depleting all four from the nucleus at the same time has a bigger effect on nucleosome spacing than just depleting Isw1 and Chd1 (Kubik et al, 2019). Positioning of the +1 nucleosome by INO80 and ISW 2 may also play a role in their control of nucleosome spacing.

Besides controlling the dynamics of nucleosome positioning, remodelers also influence TF-DNA binding dynamics. Remodelers can promote binding of TFs indirectly, for example, by removing nucleosomes that occlude TF binding sites. This was shown for the GTF Rap1, which cooperates with RSC to free Rap1 binding sites and allow for stable Rap1 binding to unoccupied DNA (Mivelaz et al, 2020). For another TF, Ace1, RSC was shown to increase binding to DNA, likely by transiently exposing Ace1 binding sites and thereby increasing the on-rate (Karpova et al, 2004; Mehta et al, 2018). Thus, chromatin remodelers play an important role in regulating the binding dynamics of both nucleosomes and TFs. 


\section{Nucleosomes are dynamically modified}

Another dynamic aspect of nucleosomes are histone modifications. Histones have unstructured N-terminal tails that protrude from the nucleosome core particle and these tails can beheavily modified post-translationally (reviewed in Kouzarides, 2007). These modifications are dynamic and are constantly being added and removed by specific histone modifiers. Some modifications strongly correlate with transcription (Pokholok et al, 2005), which suggests that they might be instructive for transcription. However, in yeast complete removal of some of these modification has surprisingly specific rather than global effects on transcription (Venkatasubrahmanyam et al, 2007; Margaritis et al, 2012; Vlaming \& van Leeuwen, 2016). Whether correlations between chromatin marks and transcription in yeast are cause, effect or due to other factors is still under debate (Howe et al, 2017; Murray et al, 2019). Some modifications may also influence nucleosome dynamics. For example, acetylation of lysine residues neutralizes their positive charge, which results in reduced electrostatic interactions with the negatively charged DNA and reduced compaction of chromatin (BrowerToland et al, 2005; Shogren-Knaak et al, 2006). Acetylation of specific lysines of H3 strongly correlates with replication-independent turnover (Rufiange et al, 2007), indicating that some modifications may also be involved in the dynamic binding of nucleosomes.

\section{Different strategies of dynamic gene regulation}

In terms of dynamic transcription regulation, there is often a distinction made between genes whose activity is dynamically regulated and genes that are more constitutively active. Constitutively active genes are often needed for basic cell survival and are expressed at similar levels between different cell types or across different conditions. They are therefore also often referred to as "housekeeping" genes (Eisenberg \& Levanon, 2013). Since their expression levels are relatively constant between different tissues and samples, housekeeping genes are often used as internal standards to calibrate gene expression measurements. There are several characteristics that differ between housekeeping and regulated genes (Cairns, 2009), which may contribute to differences in their regulatability.

Housekeeping genes are defined by a relatively "open" chromatin architecture in yeast (Tirosh \& Barkai, 2008; Field et al, 2008), which means that most housekeeping genes have a clear and wide NFR (Fig 2). This more open promoter architecture is also found in human housekeeping genes (Levitsky et al, 2001; Ganapathi et al, 2005) and may at least partially be caused by bigger poly(dA:dT) stretches. However, "open promoter" is a bit of a misnomer, since these larger NFRs contain fragile nucleosomes (Weiner et al, 2010; Xi et al, 2011; Kubik et al, 2015). Nevertheless, TFs likely have easier access to these promoters. Besides an open promoter, housekeeping genes often contain TATA-like elements rather than a TATA-box and are generally more dependent on TFIID than SAGA to bring TBP to the promoter (Lee et al, 2000; Basehoar et al, 2004; Huisinga \& Pugh, 2004; Rhee \& Pugh, 2012). In addition, housekeeping genes tend to be less sensitive to deletion of non-essential chromatin remodelers (Basehoar et al, 2004; Tirosh \& Barkai, 2008), indicating that they are regulated to a lesser extent by chromatin remodelers. 

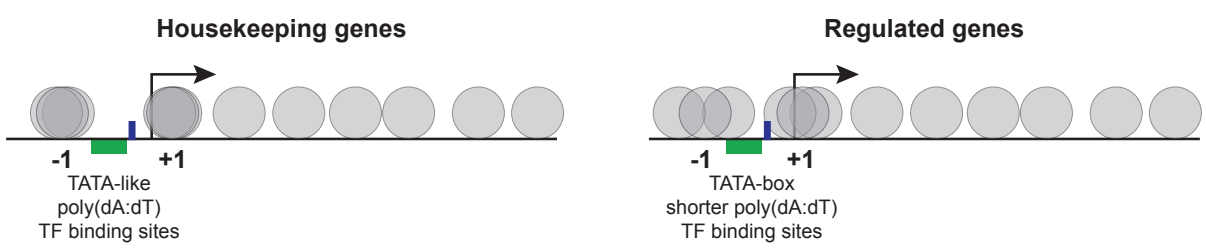

Figure 2. Typical promoter structure of regulated and housekeeping genes

Housekeeping genes generally have a well-defined NFR (green area), with poly(dA:dT) tracts and flanked by clearly positioned +1 and -1 nucleosomes. Regulated genes have a less well defined NFR, and the positioning of the +1 and -1 nucleosomes are less uniform between genes. Housekeeping genes frequently have a TATA-like element and regulated genes more often have a canonical TATA-box (dark blue). The arrow denotes the transcription start site. Adapted from (Rando \& Winston, 2012).

Regulated genes, sometimes referred to as developmentally- or stress-regulated genes, tend to be activated in response to developmental or environmental signals. These regulated genes have a chromatin organization that is different from housekeeping genes (Fig 2) and they contain more "closed" or occupied promoters (Field et al, 2008; Tirosh \& Barkai, 2008). As a group, nucleosome positioning is less uniform across regulated genes and nucleosome positioning between different cells in a population is also more delocalized (Ioshikhes et al, 2006; Albert et al, 2007; Field et al, 2008; Tirosh \& Barkai, 2008; Weiner et al, 2010). Regulated genes are therefore more dependent on chromatin remodelers for proper positioning of the +1 nucleosome (Nocetti \& Whitehouse, 2016) and efficient expression (Basehoar et al, 2004; Tirosh \& Barkai, 2008). In contrast to housekeeping genes, regulated genes are enriched for having a canonical TATA-box and are more dependent on SAGA than TFIID for bringing TBP to the promoter (Lee et al, 2000; Basehoar et al, 2004; Huisinga \& Pugh, 2004; Rhee \& Pugh, 2012).

Differences in the dependence on SAGA or TFIID, as well as the presence of a canonical TATA-box have often been used to classify genes as either regulated or housekeeping. As mentioned before, in yeast the majority of genes ( 85\%) relies more on TFIID and has a TATA-like element whereas a minority $(\sim 15 \%)$ relies more on SAGA and has a TATA-box (Lee et al, 2000; Basehoar et al, 2004; Huisinga \& Pugh, 2004; Rhee \& Pugh, 2012; Donczew et al, 2020). It is important to point out that these differences, as well as differences in promoter architecture, are not absolute and many promoters display a mix of different attributes. Importantly, this also does not mean that housekeeping genes cannot be dynamically regulated at all. The molecular mechanisms that determine differences in regulatability between housekeeping and regulated genes are largely unknown.

\section{Dynamic gene expression is regulated by dynamic TF binding}

Gene expression and protein abundance are often studied on a population level, thereby overlooking the possibility of heterogeneity or noise between single cells (Raser \& O'Shea, 2005). Although noise scales with abundance (Dar et al, 2016), the extent of gene expression noise differs between regulated and housekeeping genes. Even when normalized for abundance, protein levels of housekeeping genes have less noise compared to regulated genes (Bar-Even et al, 2006; Newman et al, 2006; 
Hornung et al, 2012). This suggests that these gene classes have different forms of regulation. Noise scaling with abundance can be explained by a two-state model of transcription, where a gene is either on or off and genes switch between these states in a stochastic manner (Raser \& O'Shea, 2004; Raj et al, 2006; Zenklusen et al, 2008). The difference in noise between regulated and housekeeping genes therefore may be caused by differences in switching between these states.

Expression for many genes does not take place continuously, but rather in bursts. Bursts have different characteristics such as how often a burst is initiated (burst frequency), how long a burst lasts (burst duration) and the number of RNA molecules that are produced in a single burst (burst size) (Lenstra et al, 2016). Genes that are noisier are also more "bursty", i.e. they have infrequent but large bursts, leading to bigger variation between cells. Measuring individual mRNA molecules in yeast showed that expression of housekeeping genes is best described by small and frequent bursts, and thus more constitutive expression. In contrast, a regulated gene is better described by bursting, with larger infrequent bursts (Zenklusen et al, 2008). This indicates that there are differences in bursting between housekeeping and regulated genes.

The presence of a TATA-box is an important regulator of noise (Bar-Even et al, 2006; Newman et al, 2006) and mutating the TATA-box to a TATA-like element significantly reduces noise (Raser \& O'Shea, 2004; Blake et al, 2006; Hornung et al, 2012). Besides the TATA-box, the local chromatin architecture, TF concentration and even genomic position can have an effect on noise and burstiness (Tirosh \& Barkai, 2008; Batenchuk et al, 2011; Hornung et al, 2012; Carey et al, 2013; Larson et al, 2013). Differences in nucleosome positioning (Field et al, 2008; Tirosh \& Barkai, 2008) and canonical TATA-box presence (Basehoar et al, 2004; Rhee \& Pugh, 2012) could explain the difference in noise between regulated and housekeeping genes. Promoters with higher noise were shown to be more responsive to environmental signals, explaining why regulated promoters have more noise (Blake et al, 2006).

The dynamics of TF-DNA interactions are important regulators of the dynamic properties of transcription. For example, the POL1 gene was suggested to be regulated mainly by TF binding frequency thereby tuning the burst frequency (Larson, 2011). Direct visualization of both TF binding and transcriptional activation of the galactose responsive gene GAL10, showed that the binding dynamics of the TF Gal4 directly determines the GAL10 bursting kinetics (Donovan et al, 2019b). In addition, this study showed that mutations in the Gal4 binding site directly affected the Gal4 residence time at a different target gene, GAL3, as well as GAL3 burst duration. In humans, acetylation of p53 has been shown to directly regulate p53 residence time and burst duration of its target genes (Loffreda et al, 2017). Thus, in a simplistic view, the TF on-rate, or frequency of binding, regulates the burst frequency. Conversely, the offrate or residence time regulates the amount of Pol II that is recruited and therefore the burst duration and burst size (Brouwer \& Lenstra, 2019). This emphasizes that measuring TF binding dynamics is crucial for understanding how transcription is dynamically regulated. 


\section{Methods to detect protein-DNA interactions}

To determine which genes are regulated by specific TFs it is important to quantify where TFs are bound in the genome. Over the years, many methods have been developed that measure protein DNA-interactions. Although in vitro methods are excellent in determining DNA binding preferences of TFs (Garner \& Revzin, 1981; Ellington \& Szostak, 1990; Tuerk \& Gold, 1990; Bulyk et al, 2001), they cannot take into account the native environment which differs, for example by frequently restricting binding of proteins to DNA. Therefore, in vivo methods are needed to determine the sites that are bound in a native setting. The first methods mainly measured binding levels to known sites or sequences. However, with the advent of microarray and sequence technologies it became possible to investigate binding to all locations across an entire genome simultaneously.

One of the oldest and most commonly used techniques to measure in vivo proteinDNA interactions directly is chromatin immunoprecipitation (ChIP). With ChIP, a protein of interest, which may or may not contain an affinity tag, is usually crosslinked to DNA (Fig 3A). Cross-linking traps protein-DNA interactions, effectively making a snapshot of the protein-DNA binding landscape. Although the first ChIP protocol used UV-crosslinking (Gilmour \& Lis, 1984), most protocols use formaldehyde to cross-link protein-DNA interactions. However, cross-linking can be omitted as well (Hebbes et al, 1988; O’Neill \& Turner, 2003; Kasinathan et al, 2014). Cells are subsequently lysed and the recovered chromatin is fragmented through sonication or enzymatic digestion. The protein of interest is next immunoprecipitated using an antibody that either recognizes the protein of interest or the affinity tag. DNA that is not bound by the protein of interest is washed away and DNA that is bound can be recovered and sequenced. Over the years, many labs have adapted the ChIP protocol, leading to many versions of the method (O'Neill \& Turner, 2003; Rhee \& Pugh, 2011; Kasinathan et al, 2014; Skene \& Henikoff, 2015; He et al, 2015; Gutin et al, 2018). These optimizations have focused on increased sensitivity and signal with little effort put into optimizing ChIP to obtain quantitatively comparable measurements between samples. Little is therefore known about which steps are needed to make the protocol more quantitative.

An alternative for ChIP to determine in vivo protein binding positions is DamID (van Steensel \& Henikoff, 2000). With DamID, the protein of interest is fused to an adenine methyltransferase (Dam). Dam methylates adenines that are part of GATC motifs in close proximity to where the protein of interest is bound (Fig 3B). After disruption of the cells and purification of DNA, methylated sequences can be enriched by digestion with DpnI, which is a methyl-sensitive restriction enzyme. By specifically ligating sequencing adapters to DpnI-digested fragments, the methylated fragments can be quantified (Vogel et al, 2007). An advantage of using DamID is that is does not require cross-linking, which is a major source of artefacts in ChIP (Park et al, 2013; Teytelman et al, 2013; Baranello et al, 2015). Several variants of DamID have been developed to measure various other processes besides protein-DNA binding (reviewed in Aughey et al, 2019). DamID has even been applied to quantify nuclear 
A ChIP

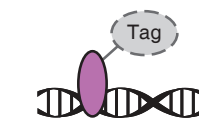

B

DamID

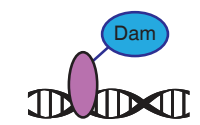

C

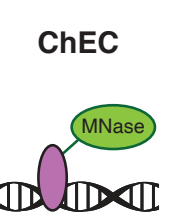

D ChIC/ CUT \& RUN

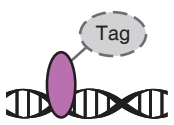

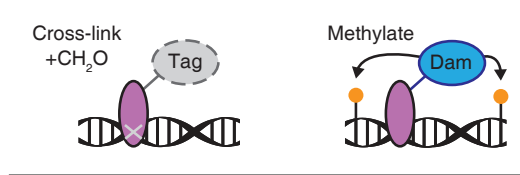

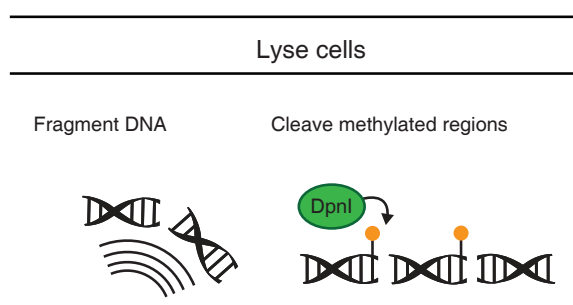

Immunoprecipitate

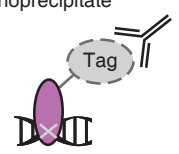

Isolate fragmented DNA and sequence

Figure 3. Schematic overview of different methods to determine protein-DNA interactions

(A) ChIP technique. The protein of interest is tagged (optional) and cross-linked to DNA. After cell lysis, DNA is fragmented through sonication or enzymatic digestion. The protein of interest and cross-linked DNA are immunoprecipitated using an antibody that recognizes the protein of interest. The bound DNA is de-cross-linked, isolated and sequenced. (B) DamID technique. The protein of interest is tagged with Dam, which methylates GATC motifs that are in close proximity (orange spheres). After cell lysis, methylated residues are cleaved using DpnI and sequenced. (C) ChEC technique. MNase is fused to the protein of interest, which is inactive in cells. After permeabilization, MNase is activated by the addition of calcium. MNase cleaves DNA in its proximity and short fragments are sequenced. (D) ChIC / CUT\&RUN technique. After permeabilization of the cells, MNase is targeted by an antibody to the protein of interest, which can contain an affinity tag. MNase is activated using calcium and small fragments that are liberated by MNase are sequenced.

lamina interactions in single cells (Kind et al, 2015). A downside of DamID is its resolution, which is lower than ChIP since it is limited by the number of and distance between GATC motifs.

Two other techniques to measure in vivo protein-DNA binding are based on targeting MNase to the protein of interest. The first technique, called chromatin endogenous cleavage (ChEC), relies on fusing MNase to the protein of interest (Schmid et al, 2004). MNase needs high levels of calcium to cleave DNA and is therefore largely inactive in living cells. Upon permeabilizing the cells and extracting nuclei, calcium is added for a short duration to activate MNase (Fig 3C). MNase will then primarily cleave DNA close to the protein of interest. By selectively sequencing short fragments, 
protein binding locations can be mapped (Zentner et al, 2015). An alternative version of ChEC, is chromatin immunocleavage (ChIC, Schmid et al, 2004). With ChIC, MNase is not directly fused, but rather indirectly targets MNase to the protein of interest using an antibody. The advantage of ChIC over ChEC is that it does not require genetic manipulation of the cells if an antibody is available. The rest of the protocol is largely the same. Cells are permeabilized, nuclei extracted and MNase, fused to an antibody that recognizes the protein of interest, can diffuse into the nuclei. MNase is subsequently activated by adding calcium. A recent genome-wide adaptation of ChIC (Fig 3D) is called CUT\&RUN (Cleavage Under Targets and Release Using Nuclease). With CUT\&RUN, the extracted nuclei are immobilized and only the small, bound and therefore cleaved fragments can diffuse out of the nuclei and will be sequenced (Skene \& Henikoff, 2017). Therefore, CUT\&RUN has very little background signal and thus requires little starting material. A version of CUT\&RUN that uses a transposase instead of MNase (CUT\&Tag) can even be used to quantify binding in single cells (Kaya-Okur et al, 2019). Strikingly, it was recently shown that there is carry-over of small amounts of bacterial DNA through the addition of MNase, which is produced in bacteria. This bacterial DNA can be used as an internal calibration of the amount of starting DNA, which allows normalization of samples with varying amounts of starting material (Meers et al, 2019).

Although such techniques, and especially ChIP, have been crucial for our understanding of transcription regulation and where proteins bind in the genome, they unfortunately only give a static snapshot of binding. Therefore, other techniques are needed to quantify protein-DNA binding dynamics.

\section{Measuring protein-DNA binding dynamics in vivo}

Microscopy-based techniques have contributed the most to our understanding of the dynamic behavior of TFs in vivo (Hager et al, 2009). Several different microscopybased techniques exist to measure TF binding dynamics. Fluorescence recovery after photobleaching (FRAP) was one of the first techniques used to study TF binding dynamics in vivo (McNally et al, 2000). With FRAP, the protein of interest is labeled with a fluorescent tag and all the fluorescent tags in a small area of the nucleus are bleached (Fig 4A). The speed of recovery of fluorescence in the bleached area is measured. By taking protein size into account, dynamics of diffusion and binding can be inferred based on how quickly the fluorescence signal recovers (Mueller et al, 2012). A related approach is fluorescence loss in photobleaching (FLIP), which constantly bleaches a single area in the nucleus while measuring loss of fluorescence in the rest of the nucleus. FLIP therefore monitors decay rather than recovery of fluorescence (McNally et al, 2000; Mueller et al, 2013). FRAP has been very powerful in measuring binding dynamics in vivo, especially because the technique is relatively straightforward and does not require complicated microscopes. Nevertheless, FRAP cannot accurately measure very transient interactions and estimates for the binding parameters are highly dependent on the model that is used to fit the data (Mueller et al, 2008). In addition, FRAP can only measure parameters for a relatively large area, which contains multiple binding locations. FRAP therefore has little power to measure dynamics for minor subpopulations of loci with different dynamics. 
A
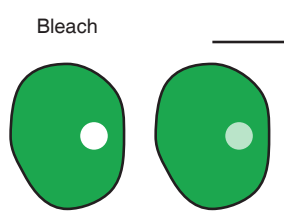

Recovery

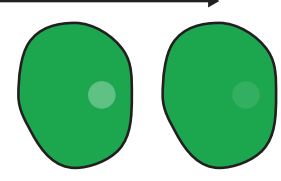

C

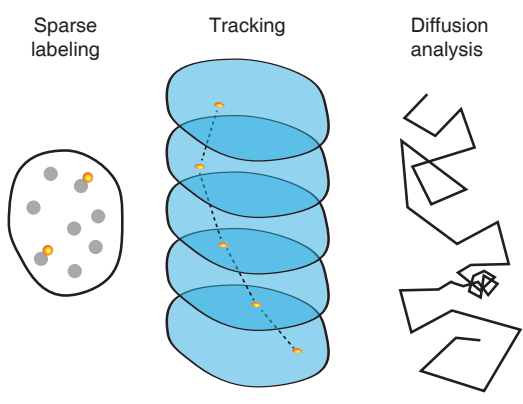

B

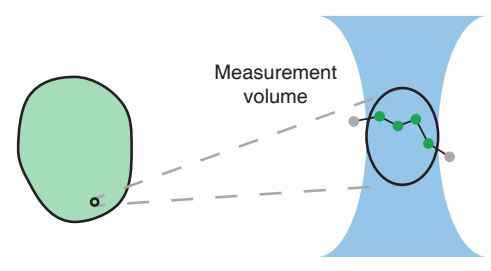

D
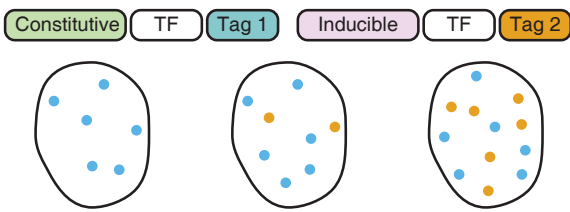

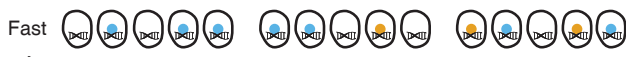

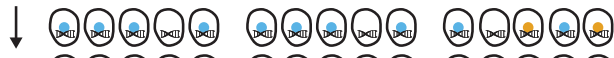

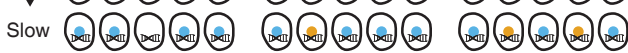

Time

Figure 4. Methods to quantify protein-DNA binding dynamics

(A) Fluorescence recovery after photobleaching (FRAP) technique. An area of the nucleus is bleached. Recovery is dependent on the speed of diffusion of unbleached fluorophores in the bleached area, as well as the dissociation rate and diffusion of bleached fluorophores out of the bleached area. The speed of recovery provides information about diffusion and residence times. (B) Fluorescence correlation spectroscopy (FCS) technique. Correlation in fluctuations of fluorescence signal over time are measured in a small area. Correlations over longer periods of time indicate slow diffusion or binding. (C) Single molecule tracking (SMT) technique. Proteins are sparsely labeled with bright fluorescent dyes to allow measurements of single molecules. Single fluorescently labeled proteins are detected and followed over time to create trajectories. From these trajectories, diffusion speed as well as the frequency and duration of binding events can be calculated. (D) Competition ChIP. Two isoforms of the protein of interest are present with different tags. Upon induction of the second tag, both isoforms will compete for the same binding sites. The increase in the binding ratio of the two tags provides information about turnover. Adapted from (Mueller et al, 2013; Zaidi et al, 2017; Liu \& Tjian, 2018).

A technique that is better suited for measuring short binding events is fluorescence correlation spectroscopy (FCS). With FCS, fluctuations in fluorescence are monitored in a small area of the nucleus over time (Fig 4B). These fluctuations are caused by fluorescently labeled molecules that diffuse in and out of the measurement area. The correlation of fluorescence between time points provides information about molecule movement. Slow moving molecules will remain longer in the measurement area and therefore stay correlated for a longer period of time. Diffusion as well as residence times can be inferred by fitting an appropriate model to the data (Michelman-Ribeiro et al, 2009). FCS is well suited for measuring fast dynamics. Variants for FCS exist that image multiple locations at the same time, allowing for a direct comparison of dynamics between different sites in the same nucleus (Hebert et al, 2005). A limitation of FCS is that it requires low concentrations of fluorescent molecules, requiring sparse labeling. In addition, non-moving molecules are quickly bleached and therefore the technique is not suited for measuring stable binding events (Stasevich et al, 2010). Finally, like FRAP, FCS is sensitive to the model used for fitting (Mazza et al, 2012). 
FRAP and FCS measure the average dynamics over an area, which masks the behavior of individual proteins. Single molecule tracking (SMT) is a technique that allows measurements of the dynamic behavior of single TFs in vivo (Fig 4C). Although single particle analysis itself is relatively old, the development of betters tools, stable dyes and more advanced microscopes has allowed for the tracking of single proteins and their binding dynamics (Liu \& Tjian, 2018). With SMT, a single fluorescently labeled protein is followed over time. By measuring its movement, both diffusion and binding can be monitored. SMT requires a low concentration of labeled protein, which can be achieved by expressing the labeled protein of interest at low levels or by using photoactivatable fluorescent proteins (Elf et al, 2007; Manley et al, 2008). Although fluorescent proteins can be used, synthetic dyes are preferred as they are more photostable (Mueller et al, 2013). Since fluorophores are bleached with repeated imaging, only a limited number of images can be taken. By adjusting the frequency of image acquisition, either fast processes such as diffusion or slow processes such as stable binding can be accurately monitored. SMT has a big advantage over other methods, since it directly measures the movement of single molecules, without making assumptions or inferring dynamics from averages. Nevertheless, it still has some limitations. It is hard to distinguish whether a molecule moved out of focus or was bleached. Therefore, it is important to correct properly for bleaching. In addition, SMT is sensitive to the imaging setup and measured residence times can differ substantially between setups (Liu \& Tjian, 2018). Nevertheless, recent advancements in SMT, combined with labeling of transcription sites, have allowed for the direct measurement of dynamics of binding and transcription at the same site simultaneously (Donovan et al, 2019b). This has enabled directly measuring how the dynamics of binding shape transcription kinetics and bursting.

The aforementioned microscopy techniques, especially SMT, excel in directly measuring TF-DNA binding dynamics, but do so either for the nucleus as a whole or for single sites. This makes studying differences in binding dynamics between binding sites of a TF with hundreds of sites technically challenging. A method that can measure binding dynamics genome-wide is competition ChIP (Fig 4D). With competition ChIP, there are two isoforms of the same protein in the cell. One is constitutively expressed under its native promoter and the other is under the control of an inducible promoter. Both isoforms have a different affinity tag, and upon induction the two isoforms will compete for binding at the same sites. By measuring the binding of both variants over time using ChIP, the turnover of the protein of interest can be monitored (Dion et al, 2007; Rufiange et al, 2007; van Werven et al, 2009; Lickwar et al, 2012). The main advantage of competition ChIP is that dynamics can be measured at all sites across the genome. However, the main limitation of competition ChIP is that the competitor is slowly induced, and takes about 20-30 minutes to be expressed. Therefore, only relatively slow turnover can be accurately quantified. In addition, competition ChIP perturbs the cells by switching the carbon source and overexpressing the protein of interest. Competition ChIP also generally only measures turnover (Dion et al, 2007; Rufiange et al, 2007; van Werven et al, 2009), whereas turnover is determined by both the on- and the off-rates. On- and 
off-rates are important to measure separately since they regulate different aspects of transcription, and because on- and off-rates are likely also regulated by different mechanisms. However, little is known about differences in on- and off-rates of the same TF between different sites in the genome, due to a lack of tools to measure them genome-wide. Therefore, additional methods are required for determining in vivo on- and off-rates of all sites across a genome.

\section{Aim and scope of the work described in this thesis}

A large body of previous research indicates the importance of dynamics in transcription regulation, yet our understanding of this aspect is quite poor. The overall aim of the work described in this thesis is to study transcription regulation dynamics. This started out with what seems an apparently simple question, namely how is the different dynamic response of housekeeping and regulatable genes achieved? Crucially for this study (chapter 2), we made use of sets of genes that depend on the exact same activator for transcription. The analyses reveal the mechanisms behind the difference in dynamics between housekeeping and regulatable genes to a great level of molecular detail. The results show how the dynamics of TF binding can have a different effect on transcription based on the molecular properties associated with different classes of promoters. Besides explaining this important difference between these two general classes of genes for the first time, the study also highlights the importance of studying TF-DNA binding dynamics for a proper molecular mechanistic understanding of cellular processes. Because of the lack of methods to properly measure TF on- and off-rates for many individual sites in parallel in vivo, a method was developed for genome-wide off-rate measurements. This method (DIVORSEQ: determining in vivo off-rates by sequencing), first required extensive optimization of one of its component parts, that is the ChIP DNA binding measurement protocol, in order to yield results quantitatively comparable between different samples. Optimization experiments for almost all individual steps of ChIP are described in chapter 3. This led to an optimized protocol, which is presented in detail in chapter 4. By applying DIVORSEQ using this optimized ChIP protocol, offrates were successfully determined genome-wide for the TF Abf1. To the best of our knowledge this is the first study to directly determine in vivo off-rates for a single TF for hundreds of sites in parallel. The results are described in chapter 5. Abf1 is a general regulatory factor in yeast, akin to chromatin pioneering TFs in larger eukaryotes, and with several previously ascribed functions, for example in organizing chromatin, regulating transcription and termination. The acquired TF-off rates cover a wide range of different values: average $\mathrm{TF}$ residence times at different loci range from 4.5 to 37 minutes. These are analyzed in the context of the different roles of Abf1. Besides showing that DIVORSEQ provides very meaningful measurements of TF binding stability, the analyses show how different binding dynamics of the same TF can contribute to different aspects of its function. The work described in this thesis demonstrates the importance of understanding TF-DNA binding dynamics in order to understand how gene expression is regulated. In addition, a powerful new tool for quantifying these dynamics is presented. The implications of this work and suggestions for future research are described in chapter 6 . 


\section{References}

Albert I, Mavrich TN, Tomsho LP, Qi J, Zanton SJ, Schuster SC, Pugh BF (2007) Translational and rotational settings of H2A.Z nucleosomes across the Saccharomyces cerevisiae genome. Nature 446: 572-576

Auble DT, Hansen KE, Mueller CG, Lane WS, Thorner J, Hahn S (1994) Mot1, a global repressor of RNA polymerase II transcription, inhibits TBP binding to DNA by an ATP-dependent mechanism. Genes Dev 8: 1920-1934

Aughey GN, Cheetham SW, Southall TD (2019) DamID as a versatile tool for understanding gene regulation. Development 146:

Badis G, Chan ET, van Bakel H, Pena-Castillo L, Tillo D, Tsui K, Carlson CD, Gossett AJ, Hasinoff MJ, Warren CL, Gebbia M, Talukder S, Yang A, Mnaimneh S, Terterov D, Coburn D, Li Yeo A, Yeo ZX, Clarke ND, Lieb JD, et al (2008) A library of yeast transcription factor motifs reveals a widespread function for Rsc3 in targeting nucleosome exclusion at promoters. Mol Cell 32: 878-887

Baranello L, Kouzine F, Sanford S, Levens D (2015) ChIP bias as a function of cross-linking time. Chromosome Res 24: 175-181

Bar-Even A, Paulsson J, Maheshri N, Carmi M, O'Shea E, Pilpel Y, Barkai N (2006) Noise in protein expression scales with natural protein abundance. Nat Genet 38: 636-643

Basehoar AD, Zanton SJ, Pugh BF (2004) Identification and distinct regulation of yeast TATA box-containing genes. Cell 116: 699-709

Batenchuk C, St-Pierre S, Tepliakova L, Adiga S, Szuto A, Kabbani N, Bell JC, Baetz K, Kærn M (2011) Chromosomal Position Effects Are Linked to Sir2-Mediated Variation in Transcriptional Burst Size. Biophys J 100: L56-L58

Blake WJ, Balázsi G, Kohanski MA, Isaacs FJ, Murphy KF, Kuang Y, Cantor CR, Walt DR, Collins JJ (2006) Phenotypic Consequences of Promoter-Mediated Transcriptional Noise. Mol Cell 24: 853-865

Bosisio D, Marazzi I, Agresti A, Shimizu N, Bianchi ME, Natoli G (2006) A hyper-dynamic equilibrium between promoter-bound and nucleoplasmic dimers controls NF- $\mathrm{B}$ dependent gene activity. EMBO J 25: 798-810

Botstein D, Fink GR (2011) Yeast: An Experimental Organism for 21st Century Biology. Genetics 189: 695-704

Brahma S, Henikoff S (2019) RSC-Associated Subnucleosomes Define MNase-Sensitive Promoters in Yeast. Mol Cell 73: 238-249.e3

Brigati C, Kurtz S, Balderes D, Vidali G, Shore D (1993) An essential yeast gene encoding a TTAGGG repeat-binding protein. Mol Cell Biol 13: 1306-1314

Brkljacic J, Grotewold E (2017) Combinatorial control of plant gene expression. Biochim Biophys Acta BBA - Gene Regul Mech 1860: 31-40

Brouwer I, Lenstra TL (2019) Visualizing transcription: key to understanding gene expression dynamics. Curr Opin Chem Biol 51: 122-129

Brower-Toland B, Wacker DA, Fulbright RM, Lis JT, Kraus WL, Wang MD (2005) Specific contributions of histone tails and their acetylation to the mechanical stability of nucleosomes. J Mol Biol 346: 135-146

Bulyk ML, Huang X, Choo Y, Church GM (2001) Exploring the DNA-binding specificities of zinc fingers with DNA microarrays. Proc Natl Acad Sci U S A 98: 7158-7163

Buratowski S, Hahn S, Guarente L, Sharp PA (1989) Five intermediate complexes in transcription initiation by RNA polymerase II. Cell 56: 549-561

Čabart P, Ujvári A, Pal M, Luse DS (2011) Transcription factor TFIIF is not required for initiation by RNA polymerase II, but it is essential to stabilize transcription factor TFIIB in early elongation complexes. Proc Natl Acad Sci U S A 108: 15786-15791

Cai M, Davis RW (1990) Yeast centromere binding protein CBF1, of the helix-loop-helix protein 
family, is required for chromosome stability and methionine prototrophy. Cell 61: 437-446

Cairns BR (2009) The logic of chromatin architecture and remodelling at promoters. Nature 461: 193-198

Cairns BR, Lorch Y, Li Y, Zhang M, Lacomis L, Erdjument-Bromage H, Tempst P, Du J, Laurent B, Kornberg RD (1996) RSC, an Essential, Abundant Chromatin-Remodeling Complex. Cell 87: 1249-1260

Candelli T, Challal D, Briand J-B, Boulay J, Porrua O, Colin J, Libri D (2018) High-resolution transcription maps reveal the widespread impact of roadblock termination in yeast. EMBO J 37: e97490

Carey LB, van Dijk D, Sloot PMA, Kaandorp JA, Segal E (2013) Promoter sequence determines the relationship between expression level and noise. PLoS Biol 11: e1001528

Chaban Y, Ezeokonkwo C, Chung W-H, Zhang F, Kornberg RD, Maier-Davis B, Lorch Y, Asturias FJ (2008) Structure of a RSC-nucleosome complex and insights into chromatin remodeling. Nat Struct Mol Biol 15: 1272-1277

Challal D, Barucco M, Kubik S, Feuerbach F, Candelli T, Geoffroy H, Benaksas C, Shore D, Libri D (2018) General Regulatory Factors Control the Fidelity of Transcription by Restricting Non-coding and Ectopic Initiation. Mol Cell 72: 955-969.e7

Chereji RV, Ocampo J, Clark DJ (2017) MNase-Sensitive Complexes in Yeast: Nucleosomes and Non-histone Barriers. Mol Cell 65: 565-577.e3

Clapier CR, Cairns BR (2009) The Biology of Chromatin Remodeling Complexes. Annu Rev Biochem 78: 273-304

Colin J, Candelli T, Porrua O, Boulay J, Zhu C, Lacroute F, Steinmetz LM, Libri D (2014) Roadblock Termination by Reb1p Restricts Cryptic and Readthrough Transcription. Mol Cell 56: 667-680

Conaway RC, Conaway JW (2011) Function and regulation of the Mediator complex. Curr Opin Genet Dev 21: 225-230

Conaway RC, Garrett KP, Hanley JP, Conaway JW (1991) Mechanism of promoter selection by RNA polymerase II: mammalian transcription factors alpha and beta gamma promote entry of polymerase into the preinitiation complex. Proc Natl Acad Sci 88: 6205-6209

Cramer P (2019) Organization and regulation of gene transcription. Nature 573: 45-54

Dar RD, Shaffer SM, Singh A, Razooky BS, Simpson ML, Raj A, Weinberger LS (2016) Transcriptional Bursting Explains the Noise-Versus-Mean Relationship in mRNA and Protein Levels. PLOS ONE 11: e0158298

Dechassa ML, Zhang B, Horowitz-Scherer R, Persinger J, Woodcock CL, Peterson CL, Bartholomew B (2008) Architecture of the SWI/SNF-Nucleosome Complex. Mol Cell Biol 28: 6010-6021

Dion MF, Kaplan T, Kim M, Buratowski S, Friedman N, Rando OJ (2007) Dynamics of replication-independent histone turnover in budding yeast. Science 315: 1405-1408

Donczew R, Warfield L, Pacheco D, Erijman A, Hahn S (2020) Two roles for the yeast transcription coactivator SAGA and a set of genes redundantly regulated by TFIID and SAGA. eLife 9: e50109

Donovan BT, Chen H, Jipa C, Bai L, Poirier MG (2019a) Dissociation rate compensation mechanism for budding yeast pioneer transcription factors. eLife 8: e43008

Donovan BT, Huynh A, Ball DA, Patel HP, Poirier MG, Larson DR, Ferguson ML, Lenstra TL (2019b) Live-cell imaging reveals the interplay between transcription factors, nucleosomes, and bursting. EMBO J 38: e100809

Eisenberg E, Levanon EY (2013) Human housekeeping genes, revisited. Trends Genet 29: 569 574

Elbi C, Walker DA, Romero G, Sullivan WP, Toft DO, Hager GL, DeFranco DB (2004) Molecular chaperones function as steroid receptor nuclear mobility factors. Proc Natl Acad Sci 101: 2876-2881 
Elf J, Li G-W, Xie XS (2007) Probing Transcription Factor Dynamics at the Single-Molecule Level in a Living Cell. Science 316: 1191-1194

Ellington AD, Szostak JW (1990) In vitro selection of RNA molecules that bind specific ligands. Nature 346: 818

Enomoto S, Longtine MS, Berman J (1994) Enhancement of Telomere-Plasmid Segregation by the X-Telomere Associated Sequence in Saccharomyces Cerevisiae Involves Sir2, Sir3, Sir4 and Abf1. Genetics 136: 757-767

Fermi B, Bosio MC, Dieci G (2016) Promoter architecture and transcriptional regulation of Abf1-dependent ribosomal protein genes in Saccharomyces cerevisiae. Nucleic Acids Res 44: 6113-6126

Field Y, Kaplan N, Fondufe-Mittendorf Y, Moore IK, Sharon E, Lubling Y, Widom J, Segal E (2008) Distinct modes of regulation by chromatin encoded through nucleosome positioning signals. PLoS Comput Biol 4: e1000216

Fuda NJ, Ardehali MB, Lis JT (2009) Defining mechanisms that regulate RNA polymerase II transcription in vivo. Nature 461: 186-192

Fukunaga K, Hirano Y, Sugimoto K (2011) Subtelomere-binding protein Tbf1 and telomerebinding protein Rap1 collaborate to inhibit localization of the Mre11 complex to DNA ends in budding yeast. Mol Biol Cell 23: 347-359

Ganapathi M, Palumbo MJ, Ansari SA, He Q, Tsui K, Nislow C, Morse RH (2011) Extensive role of the general regulatory factors, Abf1 and Rap1, in determining genome-wide chromatin structure in budding yeast. Nucleic Acids Res 39: 2032-2044

Ganapathi M, Srivastava P, Sutar SKD, Kumar K, Dasgupta D, Pal Singh G, Brahmachari V, Brahmachari SK (2005) Comparative analysis of chromatin landscape in regulatory regions of human housekeeping and tissue specific genes. BMC Bioinformatics 6: 126

Garner MM, Revzin A (1981) A gel electrophoresis method for quantifying the binding of proteins to specific DNA regions: application to components of the Escherichia coli lactose operon regulatory system. Nucleic Acids Res 9: 3047-3060

Gasch AP, Spellman PT, Kao CM, Carmel-Harel O, Eisen MB, Storz G, Botstein D, Brown PO (2000) Genomic Expression Programs in the Response of Yeast Cells to Environmental Changes. Mol Biol Cell 11: 4241-4257

Gilmour DS, Lis JT (1984) Detecting protein-DNA interactions in vivo: distribution of RNA polymerase on specific bacterial genes. Proc Natl Acad Sci U S A 81: 4275-4279

Giniger E, Varnum SM, Ptashne M (1985) Specific DNA binding of GAL4, a positive regulatory protein of yeast. Cell 40: 767-774

Gkikopoulos T, Schofield P, Singh V, Pinskaya M, Mellor J, Smolle M, Workman JL, Barton GJ, Owen-Hughes T (2011) A Role for Snf2-Related Nucleosome-Spacing Enzymes in Genome-Wide Nucleosome Organization. Science 333: 1758-1760

Görner W, Durchschlag E, Martinez-Pastor MT, Estruch F, Ammerer G, Hamilton B, Ruis H, Schüller C (1998) Nuclear localization of the C2H2 zinc finger protein Msn2p is regulated by stress and protein kinase A activity. Genes Dev 12: 586-597

Gutin J, Sadeh R, Bodenheimer N, Joseph-Strauss D, Klein-Brill A, Alajem A, Ram O, Friedman N (2018) Fine-Resolution Mapping of TF Binding and Chromatin Interactions. Cell Rep 22: 2797-2807

Haber JE (2012) Mating-Type Genes and MAT Switching in Saccharomyces cerevisiae. Genetics 191: 33-64

Hager GL, McNally JG, Misteli T (2009) Transcription dynamics. Mol Cell 35: 741-753

Hahn S, Buratowski S, Sharp PA, Guarente L (1989) Isolation of the gene encoding the yeast TATA binding protein TFIID: A gene identical to the SPT15 suppressor of Ty element insertions. Cell 58: 1173-1181

Hahn S, Young ET (2011) Transcriptional regulation in Saccharomyces cerevisiae: transcription factor regulation and function, mechanisms of initiation, and roles of activators and 
coactivators. Genetics 189: 705-736

Han Y, Reyes AA, Malik S, He Y (2020) Cryo-EM structure of SWI/SNF complex bound to a nucleosome. Nature 579: 452-455

Hansen AS, O'Shea EK (2013) Promoter decoding of transcription factor dynamics involves a trade-off between noise and control of gene expression. Mol Syst Biol 9: 704

Hao N, O'Shea EK (2012) Signal-dependent dynamics of transcription factor translocation controls gene expression. Nat Struct Mol Biol 19: 31-39

Hartley PD, Madhani HD (2009) Mechanisms that specify promoter nucleosome location and identity. Cell 137: 445-458

He Q, Johnston J, Zeitlinger J (2015) ChIP-nexus enables improved detection of in vivo transcription factor binding footprints. Nat Biotechnol 33: 395-401

Hebbes TR, Thorne AW, Crane-Robinson C (1988) A direct link between core histone acetylation and transcriptionally active chromatin. EMBO J 7: 1395-1402

Hebert B, Costantino S, Wiseman PW (2005) Spatiotemporal Image Correlation Spectroscopy (STICS) Theory, Verification, and Application to Protein Velocity Mapping in Living CHO Cells. Biophys J 88: 3601-3614

Holstege FC, van der Vliet PC, Timmers HT (1996) Opening of an RNA polymerase II promoter occurs in two distinct steps and requires the basal transcription factors IIE and IIH. ЕMBO J 15: 1666-1677

Holstege FCP, Jennings EG, Wyrick JJ, Lee TI, Hengartner CJ, Green MR, Golub TR, Lander ES, Young RA (1998) Dissecting the Regulatory Circuitry of a Eukaryotic Genome. Cell 95: $717-728$

Horikoshi M, Wang CK, Fujii H, Cromlish JA, Weil PA, Roeder RG (1989) Cloning and structure of a yeast gene encoding a general transcription initiation factor TFIID that binds to the TATA box. Nature 341: 299-303

Hornung G, Bar-Ziv R, Rosin D, Tokuriki N, Tawfik DS, Oren M, Barkai N (2012) Noise-mean relationship in mutated promoters. Genome Res 22: 2409-2417

Howe FS, Fischl H, Murray SC, Mellor J (2017) Is H3K4me3 instructive for transcription activation? BioEssays News Rev Mol Cell Dev Biol 39: 1-12

Huisinga KL, Pugh BF (2004) A genome-wide housekeeping role for TFIID and a highly regulated stress-related role for SAGA in Saccharomyces cerevisiae. Mol Cell 13: 573-585

Imbalzano AN, Zaret KS, Kingston RE (1994) Transcription factor (TF) IIB and TFIIA can independently increase the affinity of the TATA-binding protein for DNA. J Biol Chem 269: $8280-8286$

Ioshikhes IP, Albert I, Zanton SJ, Pugh BF (2006) Nucleosome positions predicted through comparative genomics. Nat Genet 38: 1210-1215

Iyer V, Struhl K (1995) Poly(dA:dT), a ubiquitous promoter element that stimulates transcription via its intrinsic DNA structure. EMBO J 14: 2570-2579

Jeronimo C, Robert F (2014) Kin28 regulates the transient association of Mediator with core promoters. Nat Struct Mol Biol 21: 449-455

Jiang C, Pugh BF (2009) Nucleosome positioning and gene regulation: advances through genomics. Nat Rev Genet 10: 161-172

Ju QD, Morrow BE, Warner JR (1990) REB1, a yeast DNA-binding protein with many targets, is essential for growth and bears some resemblance to the oncogene myb. Mol Cell Biol 10: 5226-5234

Kaplan N, Moore IK, Fondufe-Mittendorf Y, Gossett AJ, Tillo D, Field Y, LeProust EM, Hughes TR, Lieb JD, Widom J, Segal E (2009) The DNA-encoded nucleosome organization of a eukaryotic genome. Nature 458: 362-366

Karpova TS, Chen TY, Sprague BL, McNally JG (2004) Dynamic interactions of a transcription factor with DNA are accelerated by a chromatin remodeller. EMBO Rep 5: 1064-1070

Kasinathan S, Orsi GA, Zentner GE, Ahmad K, Henikoff S (2014) High-resolution mapping of 
transcription factor binding sites on native chromatin. Nat Methods 11: 203-209

Kaya-Okur HS, Wu SJ, Codomo CA, Pledger ES, Bryson TD, Henikoff JG, Ahmad K, Henikoff S (2019) CUT\&Tag for efficient epigenomic profiling of small samples and single cells. Nat Commun 10: 1-10

Kemmeren P, Sameith K, van de Pasch LAL, Benschop JJ, Lenstra TL, Margaritis T, O’Duibhir E, Apweiler E, van Wageningen S, Ko CW, van Heesch S, Kashani MM, AmpatziadisMichailidis G, Brok MO, Brabers NACH, Miles AJ, Bouwmeester D, van Hooff SR, van Bakel H, Sluiters E, et al (2014) Large-scale genetic perturbations reveal regulatory networks and an abundance of gene-specific repressors. Cell 157: 740-752

Kent NA, Eibert SM, Mellor J (2004) Cbf1p is required for chromatin remodeling at promoterproximal CACGTG motifs in yeast. J Biol Chem 279: 27116-27123

Kind J, Pagie L, de Vries SS, Nahidiazar L, Dey SS, Bienko M, Zhan Y, Lajoie B, de Graaf CA, Amendola M, Fudenberg G, Imakaev M, Mirny LA, Jalink K, Dekker J, van Oudenaarden A, van Steensel B (2015) Genome-wide Maps of Nuclear Lamina Interactions in Single Human Cells. Cell 163: 134-147

Knight B, Kubik S, Ghosh B, Bruzzone MJ, Geertz M, Martin V, Dénervaud N, Jacquet P, Ozkan B, Rougemont J, Maerkl SJ, Naef F, Shore D (2014) Two distinct promoter architectures centered on dynamic nucleosomes control ribosomal protein gene transcription. Genes Dev 28: 1695-1709

Kornberg RD (2005) Mediator and the mechanism of transcriptional activation. Trends Biochem Sci 30: 235-239

Kouzarides T (2007) Chromatin Modifications and Their Function. Cell 128: 693-705

Krietenstein N, Wal M, Watanabe S, Park B, Peterson CL, Pugh BF, Korber P (2016) Genomic Nucleosome Organization Reconstituted with Pure Proteins. Cell 167: 709-721.e12

Kubik S, Bruzzone MJ, Albert B, Shore D (2017a) A Reply to “MNase-Sensitive Complexes in Yeast: Nucleosomes and Non-histone Barriers," by Chereji et al. Mol Cell 65: 578-580

Kubik S, Bruzzone MJ, Challal D, Dreos R, Mattarocci S, Bucher P, Libri D, Shore D (2019) Opposing chromatin remodelers control transcription initiation frequency and start site selection. Nat Struct Mol Biol 26: 744-754

Kubik S, Bruzzone MJ, Jacquet P, Falcone J-L, Rougemont J, Shore D (2015) Nucleosome Stability Distinguishes Two Different Promoter Types at All Protein-Coding Genes in Yeast. Mol Cell 60: 422-434

Kubik S, Bruzzone MJ, Shore D (2017b) Establishing nucleosome architecture and stability at promoters: Roles of pioneer transcription factors and the RSC chromatin remodeler. BioEssays 39: 1600237

Kubik S, O'Duibhir E, de Jonge WJ, Mattarocci S, Albert B, Falcone J-L, Bruzzone MJ, Holstege FCP, Shore D (2018) Sequence-Directed Action of RSC Remodeler and General Regulatory Factors Modulates +1 Nucleosome Position to Facilitate Transcription. Mol Cell 71: 89-102. e5

Kuo MH, Grayhack E (1994) A library of yeast genomic MCM1 binding sites contains genes involved in cell cycle control, cell wall and membrane structure, and metabolism. Mol Cell Biol 14: 348-359

Kuras L, Kosa P, Mencia M, Struhl K (2000) TAF-Containing and TAF-Independent Forms of Transcriptionally Active TBP in Vivo. Science 288: 1244-1248

Lai WKM, Pugh BF (2017) Understanding nucleosome dynamics and their links to gene expression and DNA replication. Nat Rev Mol Cell Biol 18: 548-562

Larson DR (2011) What do expression dynamics tell us about the mechanism of transcription? Curr Opin Genet Dev 21: 591-599

Larson DR, Fritzsch C, Sun L, Meng X, Lawrence DS, Singer RH (2013) Direct observation of frequency modulated transcription in single cells using light activation. eLife 2: e00750

Lascaris RF, Mager WH, Planta RJ (1999) DNA-binding requirements of the yeast protein 
Rap1p as selected in silico from ribosomal protein gene promoter sequences. Bioinformatics 15: $267-277$

Lee TI, Causton HC, Holstege FCP, Shen W-C, Hannett N, Jennings EG, Winston F, Green MR, Young RA (2000) Redundant roles for the TFIID and SAGA complexes in global transcription. Nature 405: 701-704

Lee TI, Young RA (2013) Transcriptional Regulation and its Misregulation in Disease. Cell 152: 1237-1251

Lee W, Tillo D, Bray N, Morse RH, Davis RW, Hughes TR, Nislow C (2007) A high-resolution atlas of nucleosome occupancy in yeast. Nat Genet 39: 1235-1244

Lenburg ME, O'Shea EK (1996) Signaling phosphate starvation. Trends Biochem Sci 21: 383-387

Lenstra TL, Benschop JJ, Kim T, Schulze JM, Brabers NACH, Margaritis T, van de Pasch LAL, van Heesch SAAC, Brok MO, Groot Koerkamp MJA, Ko CW, van Leenen D, Sameith K, van Hooff SR, Lijnzaad P, Kemmeren P, Hentrich T, Kobor MS, Buratowski S, Holstege FCP (2011) The specificity and topology of chromatin interaction pathways in yeast. Mol Cell 42: 536-549

Lenstra TL, Rodriguez J, Chen H, Larson DR (2016) Transcription Dynamics in Living Cells. Annu Rev Biophys 45: 25-47

Levitsky VG, Podkolodnaya OA, Kolchanov NA, Podkolodny NL (2001) Nucleosome formation potential of eukaryotic DNA: calculation and promoters analysis. Bioinformatics 17: 998-1010

Li Y, Flanagan PM, Tschochner H, Kornberg RD (1994) RNA polymerase II initiation factor interactions and transcription start site selection. Science 263: 805-807

Lickwar CR, Mueller F, Hanlon SE, McNally JG, Lieb JD (2012) Genome-wide protein-DNA binding dynamics suggest a molecular clutch for transcription factor function. Nature 484: 251-255

Liu Q, Gabriel SE, Roinick KL, Ward RD, Arndt KM (1999) Analysis of TFIIA function In vivo: evidence for a role in TATA-binding protein recruitment and gene-specific activation. Mol Cell Biol 19: 8673-8685

Liu Z, Tjian R (2018) Visualizing transcription factor dynamics in living cells. J Cell Biol 217: $1181-1191$

Loffreda A, Jacchetti E, Antunes S, Rainone P, Daniele T, Morisaki T, Bianchi ME, Tacchetti C, Mazza D (2017) Live-cell p53 single-molecule binding is modulated by C-terminal acetylation and correlates with transcriptional activity. Nat Commun 8: 1-12

López-Maury L, Marguerat S, Bähler J (2008) Tuning gene expression to changing environments: from rapid responses to evolutionary adaptation. Nat Rev Genet 9: 583-593

Lorch Y, Maier-Davis B, Kornberg RD (2014) Role of DNA sequence in chromatin remodeling and the formation of nucleosome-free regions. Genes Dev 28: 2492-2497

Luger K, Mäder AW, Richmond RK, Sargent DF, Richmond TJ (1997) Crystal structure of the nucleosome core particle at $2.8 \AA$ resolution. Nature 389: 251-260

Luo Y, North JA, Rose SD, Poirier MG (2014) Nucleosomes accelerate transcription factor dissociation. Nucleic Acids Res 42: 3017-3027

Lustig AJ, Kurtz S, Shore D (1990) Involvement of the silencer and UAS binding protein RAP1 in regulation of telomere length. Science 250: 549-553

Manley S, Gillette JM, Patterson GH, Shroff H, Hess HF, Betzig E, Lippincott-Schwartz J (2008) High-density mapping of single-molecule trajectories with photoactivated localization microscopy. Nat Methods 5: 155-157

Marahrens Y, Stillman B (1992) A yeast chromosomal origin of DNA replication defined by multiple functional elements. Science 255: 817-823

Margaritis T, Holstege FCP (2008) Poised RNA Polymerase II Gives Pause for Thought. Cell 133: $581-584$

Margaritis T, Oreal V, Brabers N, Maestroni L, Vitaliano-Prunier A, Benschop JJ, van Hooff 
S, van Leenen D, Dargemont C, Géli V, Holstege FCP (2012) Two distinct repressive mechanisms for histone 3 lysine 4 methylation through promoting 3 '-end antisense transcription. PLoS Genet 8: e1002952

Mavrich TN, Ioshikhes IP, Venters BJ, Jiang C, Tomsho LP, Qi J, Schuster SC, Albert I, Pugh BF (2008) A barrier nucleosome model for statistical positioning of nucleosomes throughout the yeast genome. Genome Res 18: 1073-1083

Maxon ME, Goodrich JA, Tjian R (1994) Transcription factor IIE binds preferentially to RNA polymerase IIa and recruits TFIIH: a model for promoter clearance. Genes Dev 8: 515-524

Mazza D, Abernathy A, Golob N, Morisaki T, McNally JG (2012) A benchmark for chromatin binding measurements in live cells. Nucleic Acids Res 40: e119-e119

McNally JG, Müller WG, Walker D, Wolford R, Hager GL (2000) The Glucocorticoid Receptor: Rapid Exchange with Regulatory Sites in Living Cells. Science 287: 1262-1265

Meers MP, Bryson TD, Henikoff JG, Henikoff S (2019) Improved CUT\&RUN chromatin profiling tools. eLife 8: e46314

Mehta GD, Ball DA, Eriksson PR, Chereji RV, Clark DJ, McNally JG, Karpova TS (2018) SingleMolecule Analysis Reveals Linked Cycles of RSC Chromatin Remodeling and Ace1p Transcription Factor Binding in Yeast. Mol Cell 72: 875-887.e9

Messenguy F, Dubois E (2003) Role of MADS box proteins and their cofactors in combinatorial control of gene expression and cell development. Gene 316: 1-21

Michelman-Ribeiro A, Mazza D, Rosales T, Stasevich TJ, Boukari H, Rishi V, Vinson C, Knutson JR, McNally JG (2009) Direct measurement of association and dissociation rates of DNA binding in live cells by fluorescence correlation spectroscopy. Biophys J 97: 337-346

Mivelaz M, Cao A-M, Kubik S, Zencir S, Hovius R, Boichenko I, Stachowicz AM, Kurat CF, Shore D, Fierz B (2020) Chromatin Fiber Invasion and Nucleosome Displacement by the Rap1 Transcription Factor. Mol Cell 77: 488-500.e9

Mueller F, Karpova TS, Mazza D, McNally JG (2012) Monitoring dynamic binding of chromatin proteins in vivo by fluorescence recovery after photobleaching. Methods Mol Biol Clifton NJ 833: 153-176

Mueller F, Stasevich TJ, Mazza D, McNally JG (2013) Quantifying transcription factor kinetics: At work or at play? Crit Rev Biochem Mol Biol 48: 492-514

Mueller F, Wach P, McNally JG (2008) Evidence for a Common Mode of Transcription Factor Interaction with Chromatin as Revealed by Improved Quantitative Fluorescence Recovery after Photobleaching. Biophys J 94: 3323-3339

Murray SC, Lorenz P, Howe FS, Wouters M, Brown T, Xi S, Fischl H, Khushaim W, Rayappu JR, Angel A, Mellor J (2019) H3K4me3 is neither instructive for, nor informed by, transcription. bioRxiv: 709014

Neely KE, Hassan AH, Wallberg AE, Steger DJ, Cairns BR, Wright APH, Workman JL (1999) Activation Domain-Mediated Targeting of the SWI/SNF Complex to Promoters Stimulates Transcription from Nucleosome Arrays. Mol Cell 4: 649-655

Newman JRS, Ghaemmaghami S, Ihmels J, Breslow DK, Noble M, DeRisi JL, Weissman JS (2006) Single-cell proteomic analysis of S. cerevisiae reveals the architecture of biological noise. Nature 441: 840-846

Nocetti N, Whitehouse I (2016) Nucleosome repositioning underlies dynamic gene expression. Genes Dev 30: 660-672

Ocampo J, Chereji RV, Eriksson PR, Clark DJ (2016) The ISW1 and CHD1 ATP-dependent chromatin remodelers compete to set nucleosome spacing in vivo. Nucleic Acids Res 44: 4625-4635

O'Neill LP, Turner BM (2003) Immunoprecipitation of native chromatin: NChIP. Methods San Diego Calif 31: 76-82

Onodera Y, Haag JR, Ream T, Nunes PC, Pontes O, Pikaard CS (2005) Plant Nuclear RNA Polymerase IV Mediates siRNA and DNA Methylation-Dependent Heterochromatin 
Formation. Cell 120: 613-622

Papamichos-Chronakis M, Watanabe S, Rando OJ, Peterson CL (2011) Global Regulation of H2A.Z Localization by the INO80 Chromatin-Remodeling Enzyme Is Essential for Genome Integrity. Cell 144: 200-213

Park D, Lee Y, Bhupindersingh G, Iyer VR (2013) Widespread misinterpretable ChIP-seq bias in yeast. PloS One 8: e83506

Passmore S, Maine GT, Elble R, Christ C, Tye BK (1988) Saccharomyces cerevisiae protein involved in plasmid maintenance is necessary for mating of MAT alpha cells. J Mol Biol 204: 593-606

Petrenko N, Jin Y, Dong L, Wong KH, Struhl K (2019) Requirements for RNA polymerase II preinitiation complex formation in vivo. eLife 8: e43654

Pokholok DK, Harbison CT, Levine S, Cole M, Hannett NM, Lee TI, Bell GW, Walker K, Rolfe PA, Herbolsheimer E, Zeitlinger J, Lewitter F, Gifford DK, Young RA (2005) Genome-wide Map of Nucleosome Acetylation and Methylation in Yeast. Cell 122: 517-527

Porrua O, Libri D (2015) Transcription termination and the control of the transcriptome: why, where and how to stop. Nat Rev Mol Cell Biol 16: 190-202

Pryde FE, Louis EJ (1999) Limitations of silencing at native yeast telomeres. EMBO J 18: 25382550

Raj A, Peskin CS, Tranchina D, Vargas DY, Tyagi S (2006) Stochastic mRNA Synthesis in Mammalian Cells. PLOS Biol 4: e309

Rando OJ (2010) Genome-wide mapping of nucleosomes in yeast. Methods Enzymol 470: 105118

Rando OJ, Winston F (2012) Chromatin and transcription in yeast. Genetics 190: 351-387

Rani PG, Ranish JA, Hahn S (2004) RNA Polymerase II (Pol II)-TFIIF and Pol II-Mediator Complexes: the Major Stable Pol II Complexes and Their Activity in Transcription Initiation and Reinitiation. Mol Cell Biol 24: 1709-1720

Ransom M, Dennehey BK, Tyler JK (2010) Chaperoning histones during DNA replication and repair. Cell 140: 183-195

Raser JM, O'Shea EK (2004) Control of stochasticity in eukaryotic gene expression. Science 304: 1811-1814

Raser JM, O'Shea EK (2005) Noise in Gene Expression: Origins, Consequences, and Control. Science 309: 2010-2013

Ravarani CNJ, Chalancon G, Breker M, de Groot NS, Babu MM (2016) Affinity and competition for TBP are molecular determinants of gene expression noise. Nat Commun 7: 10417

Reed SH, Akiyama M, Stillman B, Friedberg EC (1999) Yeast autonomously replicating sequence binding factor is involved in nucleotide excision repair. Genes Dev 13: 3052-3058

Reja R, Vinayachandran V, Ghosh S, Pugh BF (2015) Molecular mechanisms of ribosomal protein gene coregulation. Genes Dev 29: 1942-1954

Reményi A, Schöler HR, Wilmanns M (2004) Combinatorial control of gene expression. Nat Struct Mol Biol 11: 812-815

Rhee HS, Pugh BF (2011) Comprehensive genome-wide protein-DNA interactions detected at single-nucleotide resolution. Cell 147: 1408-1419

Rhee HS, Pugh BF (2012) Genome-wide structure and organization of eukaryotic pre-initiation complexes. Nature 483: 295-301

Rhode PR, Sweder KS, Oegema KF, Campbell JL (1989) The gene encoding ARS-binding factor I is essential for the viability of yeast. Genes Dev 3: 1926-1939

Rohs R, West SM, Sosinsky A, Liu P, Mann RS, Honig B (2009) The role of DNA shape in protein-DNA recognition. Nature 461: 1248-1253

Roy K, Gabunilas J, Gillespie A, Ngo D, Chanfreau GF (2016) Common genomic elements promote transcriptional and DNA replication roadblocks. Genome Res 26: 1363-1375

Rufiange A, Jacques P-É, Bhat W, Robert F, Nourani A (2007) Genome-Wide Replication- 
Independent Histone H3 Exchange Occurs Predominantly at Promoters and Implicates H3 K56 Acetylation and Asf1. Mol Cell 27: 393-405

Sainsbury S, Bernecky C, Cramer P (2015) Structural basis of transcription initiation by RNA polymerase II. Nat Rev Mol Cell Biol 16: 129-143

Santisteban MS, Kalashnikova T, Smith MM (2000) Histone H2A.Z Regulates Transcription and Is Partially Redundant with Nucleosome Remodeling Complexes. Cell 103: 411-422

Schmid M, Durussel T, Laemmli UK (2004) ChIC and ChEC; genomic mapping of chromatin proteins. Mol Cell 16: 147-157

Segal E, Widom J (2009) From DNA sequence to transcriptional behaviour: a quantitative approach. Nat Rev Genet 10: 443-456

Shogren-Knaak M, Ishii H, Sun J-M, Pazin MJ, Davie JR, Peterson CL (2006) Histone H4-K16 Acetylation Controls Chromatin Structure and Protein Interactions. Science 311: 844-847

Skene PJ, Henikoff S (2015) A simple method for generating high-resolution maps of genomewide protein binding. eLife 4: e09225

Skene PJ, Henikoff S (2017) An efficient targeted nuclease strategy for high-resolution mapping of DNA binding sites. eLife 6: e21856

Spedale G, Meddens CA, Koster MJE, Ko CW, van Hooff SR, Holstege FCP, Timmers HTM, Pijnappel WWMP (2012) Tight cooperation between Mot1p and NC2 $\beta$ in regulating genome-wide transcription, repression of transcription following heat shock induction and genetic interaction with SAGA. Nucleic Acids Res 40: 996-1008

Stargell LA, Moqtaderi Z, Dorris DR, Ogg RC, Struhl K (2000) TFIIA has activator-dependent and core promoter functions in vivo. J Biol Chem 275: 12374-12380

Stasevich TJ, Mueller F, Michelman-Ribeiro A, Rosales T, Knutson JR, McNally JG (2010) Cross-Validating FRAP and FCS to Quantify the Impact of Photobleaching on In Vivo Binding Estimates. Biophys J 99: 3093-3101

Stavreva DA, Müller WG, Hager GL, Smith CL, McNally JG (2004) Rapid glucocorticoid receptor exchange at a promoter is coupled to transcription and regulated by chaperones and proteasomes. Mol Cell Biol 24: 2682-2697

van Steensel B, Henikoff S (2000) Identification of in vivo DNA targets of chromatin proteins using tethered dam methyltransferase. Nat Biotechnol 18: 424-428

Struhl K (1985) Naturally occurring poly(dA-dT) sequences are upstream promoter elements for constitutive transcription in yeast. Proc Natl Acad Sci U S A 82: 8419-8423

Takaoka K, Hamada H (2012) Cell fate decisions and axis determination in the early mouse embryo. Development 139: 3-14

Teytelman L, Thurtle DM, Rine J, van Oudenaarden A (2013) Highly expressed loci are vulnerable to misleading ChIP localization of multiple unrelated proteins. Proc Natl Acad Sci U S A 110: 18602-18607

Thompson CM, Young RA (1995) General requirement for RNA polymerase II holoenzymes in vivo. Proc Natl Acad Sci 92: 4587-4590

Tirosh I, Barkai N (2008) Two strategies for gene regulation by promoter nucleosomes. Genome Res 18: 1084-1091

Tirosh I, Sigal N, Barkai N (2010) Widespread remodeling of mid-coding sequence nucleosomes by Isw1. Genome Biol 11: R49

Tolkunov D, Zawadzki KA, Singer C, Elfving N, Morozov AV, Broach JR (2011) Chromatin remodelers clear nucleosomes from intrinsically unfavorable sites to establish nucleosomedepleted regions at promoters. Mol Biol Cell 22: 2106-2118

Tuerk C, Gold L (1990) Systematic evolution of ligands by exponential enrichment: RNA ligands to bacteriophage T4 DNA polymerase. Science 249: 505-510

Udugama M, Sabri A, Bartholomew B (2011) The INO80 ATP-dependent chromatin remodeling complex is a nucleosome spacing factor. Mol Cell Biol 31: 662-673

Vary JC, Gangaraju VK, Qin J, Landel CC, Kooperberg C, Bartholomew B, Tsukiyama T (2003) 
Yeast Isw1p forms two separable complexes in vivo. Mol Cell Biol 23: 80-91

Venkatasubrahmanyam S, Hwang WW, Meneghini MD, Tong AHY, Madhani HD (2007) Genome-wide, as opposed to local, antisilencing is mediated redundantly by the euchromatic factors Set1 and H2A.Z. Proc Natl Acad Sci 104: 16609-16614

Vlaming H, van Leeuwen F (2016) The upstreams and downstreams of H3K79 methylation by DOT1L. Chromosoma 125: 593-605

Vogel MJ, Peric-Hupkes D, van Steensel B (2007) Detection of in vivo protein-DNA interactions using DamID in mammalian cells. Nat Protoc 2: 1467-1478

Wagner FR, Dienemann C, Wang H, Stützer A, Tegunov D, Urlaub H, Cramer P (2020) Structure of SWI/SNF chromatin remodeller RSC bound to a nucleosome. Nature 579: 448-451

Weber CM, Ramachandran S, Henikoff S (2014) Nucleosomes are context-specific, H2A.Zmodulated barriers to RNA polymerase. Mol Cell 53: 819-830

Weiner A, Hughes A, Yassour M, Rando OJ, Friedman N (2010) High-resolution nucleosome mapping reveals transcription-dependent promoter packaging. Genome Res 20: 90-100

van Werven FJ, van Bakel H, van Teeffelen HAAM, Altelaar AFM, Koerkamp MG, Heck AJR, Holstege FCP, Timmers HTM (2008) Cooperative action of NC2 and Mot1p to regulate TATA-binding protein function across the genome. Genes Dev 22: 2359-2369

van Werven FJ, van Teeffelen HAAM, Holstege FCP, Timmers HTM (2009) Distinct promoter dynamics of the basal transcription factor TBP across the yeast genome. Nat Struct Mol Biol 16: 1043-1048

White RJ, Collins JE, Sealy IM, Wali N, Dooley CM, Digby Z, Stemple DL, Murphy DN, Billis K, Hourlier T, Füllgrabe A, Davis MP, Enright AJ, Busch-Nentwich EM (2017) A highresolution mRNA expression time course of embryonic development in zebrafish. eLife 6: e30860

Whitehouse I, Rando OJ, Delrow J, Tsukiyama T (2007) Chromatin remodelling at promoters suppresses antisense transcription. Nature 450: 1031-1035

Wong KH, Jin Y, Struhl K (2014) TFIIH phosphorylation of the Pol II CTD stimulates mediator dissociation from the preinitiation complex and promoter escape. Mol Cell 54: 601-612

Xi Y, Yao J, Chen R, Li W, He X (2011) Nucleosome fragility reveals novel functional states of chromatin and poises genes for activation. Genome Res 21: 718-724

Yao J, Munson KM, Webb WW, Lis JT (2006) Dynamics of heat shock factor association with native gene loci in living cells. Nature 442: 1050-1053

Yen K, Vinayachandran V, Batta K, Koerber RT, Pugh BF (2012) Genome-wide nucleosome specificity and directionality of chromatin remodelers. Cell 149: 1461-1473

Yen K, Vinayachandran V, Pugh BF (2013) SWR-C and INO80 Chromatin Remodelers Recognize Nucleosome-free Regions Near +1 Nucleosomes. Cell 154: 1246-1256

Yi H, Xue L, Guo M-X, Ma J, Zeng Y, Wang W, Cai J-Y, Hu H-M, Shu H-B, Shi Y-B, Li W-X (2010) Gene expression atlas for human embryogenesis. FASEB J 24: 3341-3350

Yuan G-C, Liu Y-J, Dion MF, Slack MD, Wu LF, Altschuler SJ, Rando OJ (2005) Genome-Scale Identification of Nucleosome Positions in S. cerevisiae. Science 309: 626-630

Yudkovsky N, Logie C, Hahn S, Peterson CL (1999) Recruitment of the SWI/SNF chromatin remodeling complex by transcriptional activators. Genes Dev 13: 2369-2374

Zaidi HA, Auble DT, Bekiranov S (2017) RNA synthesis is associated with multiple TBPchromatin binding events. Sci Rep $7: 1-12$

Zaret KS, Carroll JS (2011) Pioneer transcription factors: establishing competence for gene expression. Genes Dev 25: 2227-2241

Zenklusen D, Larson DR, Singer RH (2008) Single-RNA counting reveals alternative modes of gene expression in yeast. Nat Struct Mol Biol 15: 1263-1271

Zentner GE, Kasinathan S, Xin B, Rohs R, Henikoff S (2015) ChEC-seq kinetics discriminates transcription factor binding sites by DNA sequence and shape in vivo. Nat Commun 6: 


\section{Chapter I}

8733

Zhang H, Roberts DN, Cairns BR (2005) Genome-wide dynamics of Htz1, a histone H2A variant that poises repressed/basal promoters for activation through histone loss. Cell 123: 219-231

Zhou T, Yang L, Lu Y, Dror I, Dantas Machado AC, Ghane T, Di Felice R, Rohs R (2013) DNAshape: a method for the high-throughput prediction of DNA structural features on a genomic scale. Nucleic Acids Res 41: W56-62

Zhu F, Farnung L, Kaasinen E, Sahu B, Yin Y, Wei B, Dodonova SO, Nitta KR, Morgunova E, Taipale M, Cramer P, Taipale J (2018) The interaction landscape between transcription factors and the nucleosome. Nature 562: 76-81 


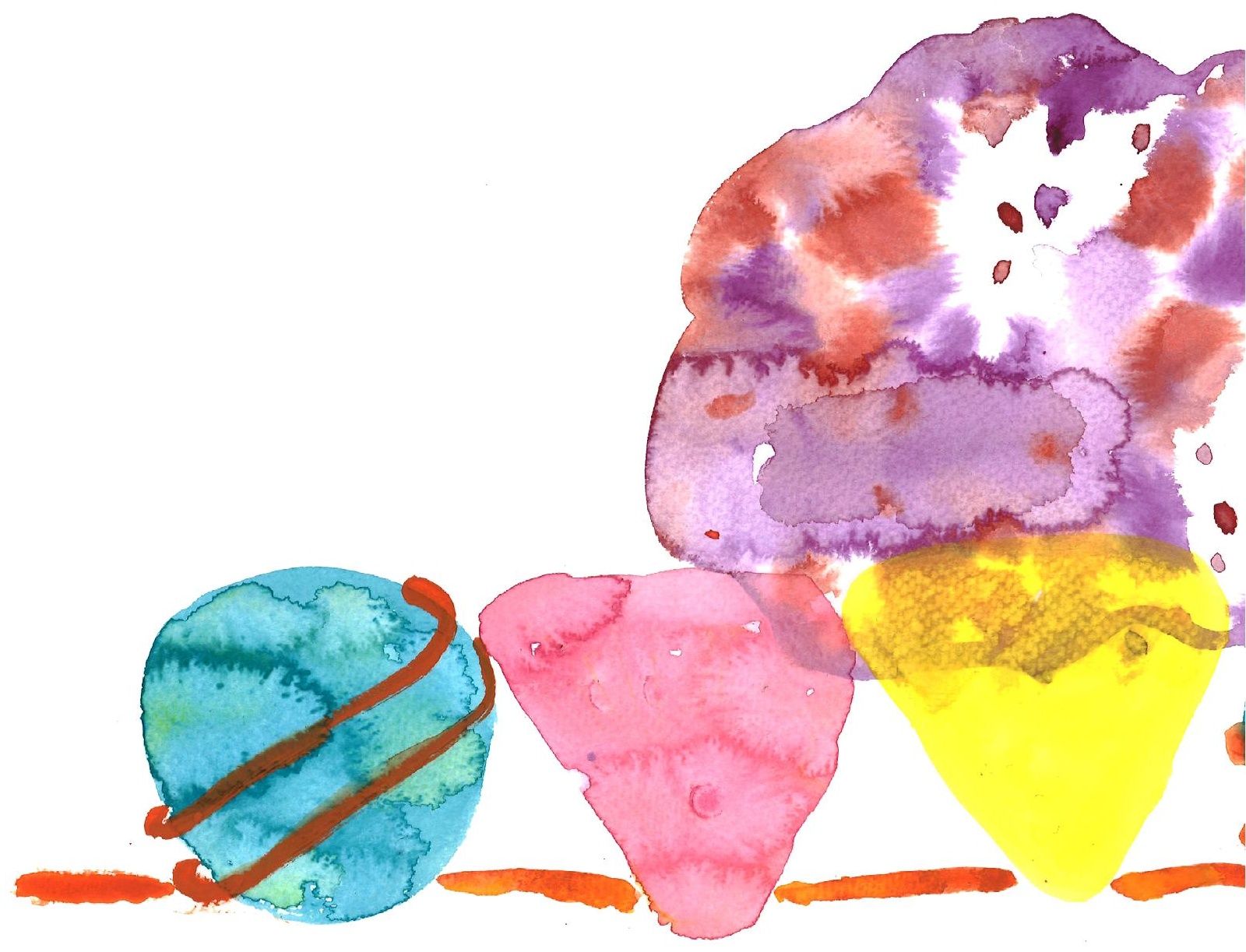




\section{Abstract}

An important distinction is frequently made between constitutively expressed housekeeping genes versus regulated genes. Although generally characterized by different DNA elements, chromatin architecture and cofactors, it is not known to what degree promoter classes strictly follow regulatability rules and which molecular mechanisms dictate such differences. We show that SAGA-dominated/ TATA-box promoters are more responsive to changes in the amount of activator, even compared to TFIID/TATA-like promoters that depend on the same activator Hsf1. Regulatability is therefore an inherent property of promoter class. Further analyses show that SAGA/TATA-box promoters are more dynamic because TBP recruitment through SAGA is susceptible to removal by Mot1. In addition, the nucleosome configuration upon activator depletion shifts on SAGA/TATA-box promoters and seems less amenable to preinitiation complex formation. The results explain the fundamental difference between housekeeping and regulatable genes, revealing an additional facet of combinatorial control: an activator can elicit a different response dependent on core promoter class. 


\section{Introduction}

First coined almost 40 years ago, the term "housekeeping gene" is widely used to distinguish genes with constitutive expression from genes whereby expression is regulated upon environmental, cell type or developmental cues. Genome-wide analyses have facilitated the identification of housekeeping genes, resulting in the characterisation of general properties such as gene structure, evolutionary conservation and promoter features (Eisenberg \& Levanon, 2013). Despite the longstanding distinction that has been made between housekeeping and regulatable genes, the degree to which such a functional division strictly applies is not clear. In addition, the molecular mechanisms behind such functional differences are not understood.

As well as being grouped based on function, genes can also be classified according to the DNA elements present in their promoters. Besides cis-regulatory elements for binding transcriptional activators, promoters also harbour core promoter elements (Juven-Gershon \& Kadonaga, 2010; Müller \& Tora, 2014). One such core promoter motif is the TATA-box. The presence or absence of such core promoter elements has been linked to function. For example mammalian genes without a welldefined TATA-box but with a CPG island promoter, are generally associated with housekeeping function (Deaton \& Bird, 2011). Functional distinction based on core promoter type is not clear-cut however. For example, many tissue-specific genes are also driven by CpG island promoters (Deaton \& Bird, 2011).

A less complex regulatory system is offered by the yeast Saccharomyces cerevisiae where a similar dichotomy is present. Here promoters with a TATA-like element rather than a consensus TATA-box are also generally associated with house-keeping function (Basehoar et al, 2004; Huisinga \& Pugh, 2004). A related dichotomy is based on the co-activator used to nucleate assembly of the RNA polymerase II preinitiation complex (PIC) at the different core promoter types (Huisinga \& Pugh, 2004; Lee et al, 2000). An essential and early step in PIC formation is TATA-binding protein (TBP) recruitment (Buratowski et al, 1989; Davison et al, 1983; Roeder, 1996). Whereas TATA-box containing promoters generally depend on the co-activator SAGA to recruit TBP, TATA-like promoters are enriched for the co-activator TFIID for TBP recruitment (Basehoar et al, 2004; Huisinga \& Pugh, 2004; Rhee \& Pugh, 2012).

In yeast there is therefore a general distinction between housekeeping genes with a TATA-like element and preferential usage of TFIID on the one hand, versus regulatable/responsive genes with a consensus TATA-box and preferential usage of SAGA on the other. A clear explanation for the regulatory difference between these two broad classes of promoters is missing however. Several concepts are possible. For example, on average, individual SAGA dominated/TATA-box promoters seem to be targeted by more regulators than TFIID dominated/TATA-like promoters (Huisinga \& Pugh, 2004; Tirosh et al, 2006; Venters et al, 2011) offering a possible straightforward explanation for their higher regulatability. There are also general differences in nucleosome configuration between the two promoter classes (Albert et 
al, 2007; Cairns, 2009; Jiang \& Pugh, 2009; Rhee \& Pugh, 2012; Tirosh \& Barkai, 2008). Nucleosome occupancy and positioning differences have previously been associated with diverse regulatory properties including responsiveness (Lam et al, 2008; RavehSadka et al, 2009; Tirosh \& Barkai, 2008). Whether chromatin architecture explains regulatability differences between SAGA- and TFIID-dominated promoters is still an open question however (Nocetti \& Whitehouse, 2016). Furthermore, neither the involvement of chromatin architecture nor a difference in the number of regulators explain the apparent involvement of the two coactivators TFIID and SAGA in regulatability differences. This is particularly poignant given that an association between TATA-box type and constitutive versus regulatable expression was first reported 30 years ago (Struhl, 1986).

Here we characterize differences in regulatability between SAGA and TFIID dominated genes and study the molecular mechanisms that dictate this property. Most of what is known about the relationship between core promoter type and responsiveness is derived from correlations between genome-scale datasets. We therefore first set up a tractable experimental system that allows for a clear measure of promoter responsiveness, is amenable to mechanistic analyses, and is capable of addressing the role of core promoter type with minimal potential interference of different regulator use. By focusing on a single transcriptional activator that serves genes from both classes of promoters, a clear difference in regulatability is demonstrated. Whereas SAGA/TATA-box promoters are highly responsive to the presence of the activator Hsf1, TFIID/TATA-like promoters are much less dynamically responsive to changes of the same activator. On its own, this clearly establishes that there is indeed an inherent difference in regulatability between the two promoter classes, not dictated by use of different numbers or types of transcriptional activators. The system is then further analysed to uncover the molecular mechanisms that dictate the difference in responsiveness. Evidence for two contributing mechanisms is presented. The first explains the differential involvement of TFIID and SAGA on constitutive and responsive promoters respectively, and involves a general negative regulator of TBP binding, Mot1 (Pereira et al, 2003). The second mechanism involves differences in the nucleosome architecture changes observed upon activator absence or presence on the two promoter classes. The consequence of both mechanisms is that promoter output on SAGA/TATA-box promoters is more dynamically dependent on the presence of an activator because of dynamic negative regulation particular to this promoter class. The results reveal an additional facet of combinatorial control and provide a molecular mechanistic explanation for the fundamental difference between house-keeping and regulatable genes.

\section{Results}

Previously reported differences in responsiveness between SAGA and TFIID dominated genes, or between TATA-box and TATA-like promoters, were based on general observations of higher variation in expression levels for SAGA dominated/ TATA-like genes under a variety of experimental and genetic perturbations (Huisinga 
\& Pugh, 2004; Tirosh et al, 2006; Venters et al, 2011). Such differences may simply reflect a difference between the type or number of transcriptional regulators used by the two classes of promoters, as has indeed been suggested (Huisinga \& Pugh, 2004; Tirosh et al, 2006; Venters et al, 2011). We therefore first determined whether there is a transcriptional activator that regulates genes from both promoter classes. Based on genome-wide binding of most yeast transcription factors (MacIsaac et al, 2006), a possible candidate is Heat Shock Factor 1 (Hsf1). Cofactor binding data indicates that approximately half of the promoters bound by Hsf1 are dominated by SAGA, and the other half by TFIID (Rhee \& Pugh, 2012). This suggests that Hsf1 and its targets would likely form a good model to investigate inherent differences in regulatability between SAGA and TFIID dominated promoters. Since this assessment is based on genome-wide binding data, it was important to first verify which targets are indeed dependent on Hsf1, and whether these direct targets represent both classes of core promoters.

\section{Rapid nuclear depletion of Hsf1}

Hsf1 is required for the induction of heat shock proteins but also binds some promoters under standard growth conditions (Hahn et al, 2004). This is likely the reason why HSF1 is essential for cell viability even in the absence of a heat shock (Wiederrecht et al, 1988), a proposal that has recently been confirmed (Solís et al, 2016). Previous studies have mostly used temperature-sensitive HSF1 mutant alleles to study Hsf1 function (Imazu \& Sakurai, 2005; Smith \& Yaffe, 1991; Zarzov et al, 1997). To circumvent the secondary effects associated with such approaches, an inducible Hsf1 nuclear depletion strain was constructed based on the "anchoraway" technique developed in the Laemmli lab (Haruki et al, 2008), but using the S288C/BY4742 genetic background. Besides the FRB (FK506 binding proteinrapamycin binding domain) tag required to induce nuclear depletion, GFP was also added to monitor cellular location. To ensure that FRB-GFP tagging of Hsf1 does not interfere with its function, growth of the tagged strain was compared to an untagged background strain (wildtype: WT). Growth was found to be identical (Fig 1A). As a more stringent test, genome-wide mRNA synthesis was compared between WT and the tagged strain (Fig 1B). Only three genes show a significant difference in mRNA synthesis $(p<0.01$ and fold change $(\mathrm{FC})>1.7)$. The two genes with apparently decreased levels are HSF1 itself and a dubious open reading frame (ORF) overlapping the altered 3' end of HSF1. The HSF1 gene is considerably longer because of the FRB-GFP tag, making cDNA synthesis and cRNA amplification of the region encompassing the microarray probe less efficient and thereby causing an apparent drop in transcription rate compared to WT. The gene with increased transcription is the dubious ORF YDL196W, known to have highly variable expression in WT strains (Kemmeren et al, 2014). The small number of expression changes (Fig 1B) and identical growth compared to WT (Fig 1A), indicates that tagging of Hsf1 in this way doesn't compromise its function under standard growth conditions.

Yeast Hsf1 is a nuclear protein (Morano et al, 2012). Anchor-away with the FRBGFP tagged strain facilitates induced nuclear depletion. HSF1 FRB-GFP tagged cells cease growth approximately five hours after starting depletion (Fig 1A). 
A

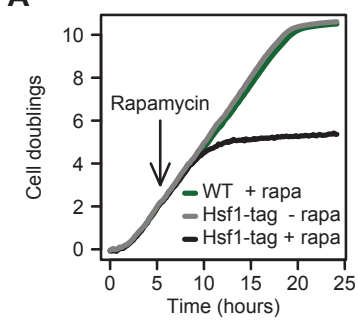

D

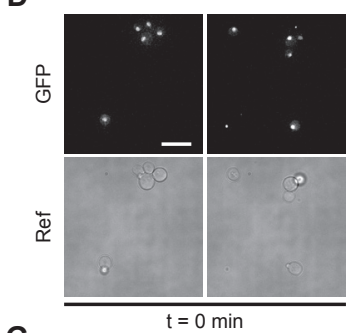

G

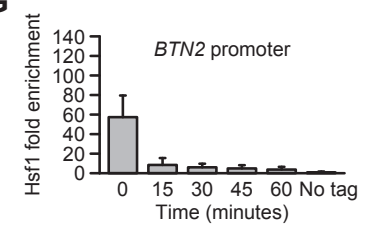

I

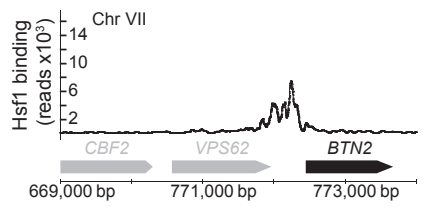

B

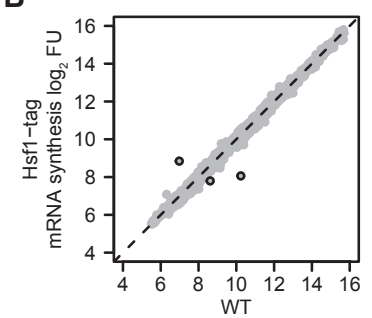

E

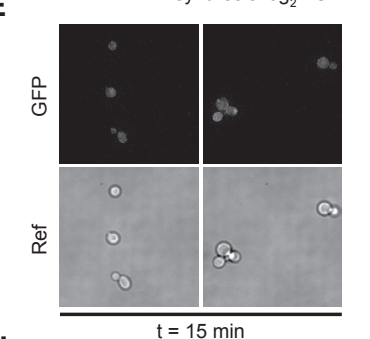

H

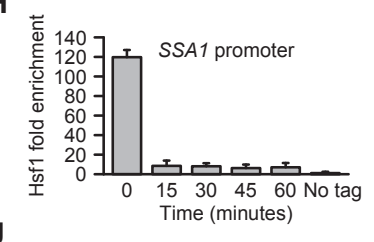

$\mathrm{J}$

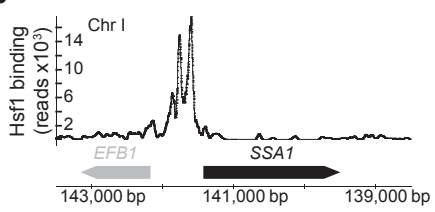

C

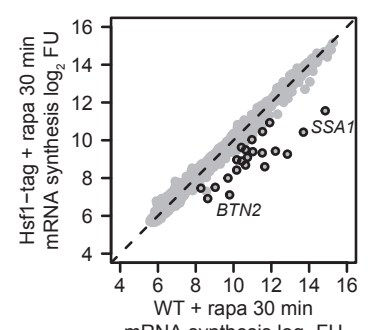

$\mathbf{F}$

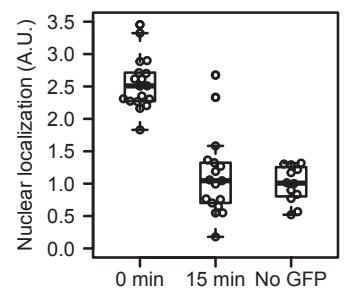

Figure 1. Dynamics of Hsf1 nuclear depletion

(A) Growth curves of WT and the HSF1 FRB-GFP tagged strain with and without rapamycin, the inducing agent for nuclear depletion (Haruki et al., 2008). WT is the parental S288C/BY4742 strain for anchor away that has been genetically desensitized to rapamycin. (B) Scatterplot of 4tU-derived mRNA synthesis in the Hsf1 tagged strain compared to WT. The $x$ - and y-axes plot the $\log _{2}$ fluorescent dye intensities of the microarray probes representing each gene (dots), averaged over four replicates. Black circles indicate genes with significantly altered synthesis ( $\mathrm{FC}>1.7$ and $p<0.01$, calculated using limma). (C) mRNA synthesis, 30 minutes after induction of Hsf1 nuclear depletion versus same treatment WT. Black circles indicate genes with significantly decreased synthesis (FC $>1.7$ and $p<0.01$, calculated using limma). (D) Fluorescence microscopy images of Hsf1-FRB-GFP at $\mathrm{t}=0$ for induced depletion. Scale bar: 10 $\mu \mathrm{m}$. (E) Fluorescence microscopy images of Hsf1-FRB-GFP 15 minutes after induction of depletion. (F) Boxplot showing the quantification of Hsf1 depletion from the nucleus. Dots represent individual cell measurements. ( $\mathbf{G}$ and $\mathbf{H}$ ) Dynamics of Hsf1 depletion from the SSA1 promoter (SAGA dominated) and BTN2 promoter (TFIID dominated), as measured by ChIP-qPCR. Error bars show the standard deviation of four independent replicates. (I and J) Hsf1 binding to the SSA1 and BTN2 promoters as measured by ChIP-seq.

This agrees with the previously established essential role of Hsf1 under standard growth conditions (Wiederrecht et al, 1988). Given that it is the lack of expression of target genes that causes HSF1 gene deletion lethality (Solís et al, 2016), growth 
cessation is an indirect measure of Hsf1 loss of function dynamics. Fluorescence microscopy was therefore applied to more precisely determine the dynamics of Hsf1 nuclear depletion. In contrast to cessation of growth (five hours: Fig 1A), nuclear depletion of Hsf1 is much faster, with nuclear GFP signal dropping to background levels within 15 minutes (Fig 1D-1F), in agreement with Hsf1 nuclear depletion in a different genetic background (Solís et al, 2016). To establish whether any residual Hsf1 remains bound to target promoters after 15 minutes, at levels undetectable by fluorescence microscopy, chromatin immunoprecipitation (ChIP) was performed on two Hsf1 bound promoters (MacIsaac et al, 2006): BTN2, which is TFIID dominated and SSA1 which is SAGA dominated (Rhee \& Pugh, 2012). The dynamics of Hsf1 loss from both promoters (Fig 1G and $\mathbf{1 H}$ ) corresponds very well with the dynamics of nuclear depletion determined by fluorescence microscopy (Fig 1E and 1F), also reaching background levels after 15 minutes of depletion (Fig $\mathbf{1 G}$ and $\mathbf{1 H}$ ). Taken together, these results demonstrate that Hsf1 can be rapidly depleted from target promoters and the nucleus within 15 minutes.

\section{Direct targets of Hsf1 during standard growth conditions}

To determine the direct transcriptional targets of Hsf1, mRNA synthesis rates were monitored upon Hsf1 depletion and compared to Hsf1 genome-wide localization derived from ChIP-seq. mRNA synthesis rates were determined using 4-thiouracil $(4 \mathrm{tU})$ which is incorporated into newly synthesized mRNA's (Sun et al, 2012). This eliminates the confounding effect of differential mRNA decay rates interfering with target gene identification and is a more direct measure of promoter output, better suited to the goals of this study. A specific set of genes show reduced synthesis rates 30 minutes after induction of Hsf1 depletion $(p<0.01$ and FC $>1.7$, Fig 1C). To determine whether all these effects are direct, Hsf1 binding was monitored by ChIPseq under the same growth condition. All genes with decreased mRNA synthesis (Fig 1C) also show strong Hsf1 promoter binding. Examples of TFIID and SAGA dominated targets are shown in Fig1I-J. Integration of the mRNA synthesis and Hsf1 binding data leads to identification of 21 direct targets (Materials and Methods). These are enriched for the Gene Ontology (GO) term protein folding $\left(p<2 \times 10^{-24}\right)$, in agreement with the established role of Hsf1 in regulating the expression of protein chaperones, also under non-heat shock conditions (Gross et al, 1990; Solís et al, 2016). The 21 targets are a subset of the promoters previously reported to be bound by Hsf1 under standard and heat shock conditions (Hahn et al, 2004) and correspond almost exactly with Hsf1 direct targets also recently defined using anchor away (Solís et al, 2016). Most importantly, half of these targets are dominated by SAGA (11/21) and the other half by TFIID (10/21) (Rhee \& Pugh, 2012). This is in itself an important finding. The fact that a single activator can equally service genes from both promoter classes already indicates that different regulatability properties associated with the different classes are indeed inherent to promoter type rather than dependent on the number or type of activator. Such a conclusion can only be drawn if the Hsf1 targets actually exhibit differences in regulatability between the two promoter classes. We therefore next set out to investigate regulatability using the Hsf1 target genes as a model. 


\section{SAGA and TFIID dominated genes are dependent on Hsf1 in different ways}

To serve as a model, it is also essential that the Hsf1 direct targets exhibit the properties that are characteristic for each promoter class (Albert et al, 2007; Cairns, 2009; Rhee \& Pugh, 2012; Tirosh \& Barkai, 2008). TFIID dominated promoters are characterized by a nucleosome depleted region (NDR) followed by a well positioned +1 nucleosome. SAGA dominated genes generally show a less uniformly organized promoter, with fuzzier nucleosome positioning and a smaller, less pronounced NDR. Based on publicly available nucleosome occupancy data (Jiang \& Pugh, 2009), the Hsf1 targets defined here reflect these established differences (Fig 2A-2D). The average location of $\mathrm{Hsf1}$ binding motifs also show a clear difference, with a location approximately $60 \mathrm{bp}$ more downstream in TFIID compared to SAGA dominated promoters (Fig 2C and 2D). A similar difference in motif location of other activators has been described before, for promoters with and without a TATA-box (Erb \& van Nimwegen, 2011). Together, the attributes displayed by the two sets of Hsf1 targets defined here, fit very well with the attributes previously defined on TFIID and SAGA dominated promoters genome-wide, further indicating their utility to study functional differences between the two classes, irrespective of activator usage.

By definition, all the 21 direct target genes of Hsf1 are dependent for mRNA synthesis on Hsf1 presence, regardless of promoter class. To investigate promoter responsiveness, we first asked whether there is a relationship between Hsf1 binding levels under standard growth conditions, and the drop in mRNA synthesis observed upon Hsf1 depletion. Strikingly, SAGA dominated genes exhibit a strong correlation between the amount of Hsf1 initially bound to the promoter and the change in transcriptional output upon Hsf1 depletion (Fig 2F, the correlation of the SAGA dominated genes is robust to outliers Table S1). This is not the case for TFIID dominated genes. Here there is no statistically significant correlation and the value depicted is dependent on a single data point (Fig 2E, Table S1). On average the Hsf1 binding levels are two-fold higher on SAGA dominated promoters. This difference is not statistically significant however ( $p=0.07$, two-sided t-test), and sequence motif analyses did not reveal strong differences either. Furthermore, although a few of the SAGA dominated promoters exhibit high initial Hsf1 binding levels, the stronger response to Hsf1 loss is also exhibited by those SAGA dominated promoters with lower initial Hsf1 binding levels (Fig 2F). These comparisons indicate that for SAGA dominated promoters there is a tight relationship between the amount of Hsf1 that is bound and the transcriptional output, whereas for TFIID dominated genes such a clear relationship is lacking. This difference fits with the idea that SAGA dominated promoters are more regulatable than TFIID dominated promoters.

\section{SAGA dominated promoters are more responsive to activator presence}

A limitation of the previous analysis is that it does not determine how each individual promoter responds to different amounts of Hsf1. To investigate the difference between TFIID and SAGA dominated Hsf1 targets in more detail, individual promoter responses were determined by measuring Hsf 1 binding and transcriptional output over a range of nuclear Hsf1 concentrations. Because depletion of Hsf1 from the nucleus is rapid (Fig 1D-1F), especially in comparison to the six minute window 

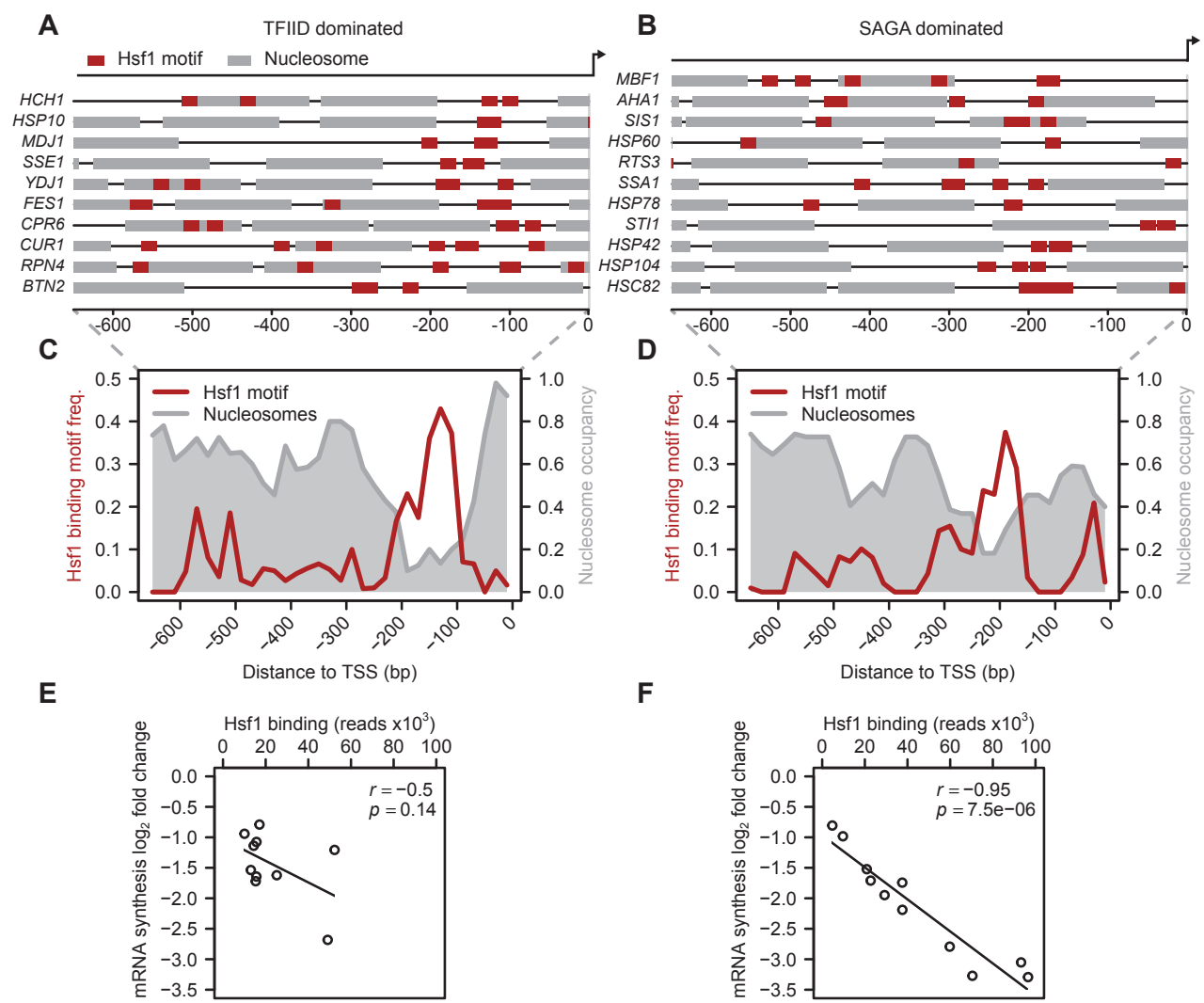

Figure 2. SAGA and TFIID dominated genes are differentially sensitive to loss of the same activator (A) Map of nucleosome positions (Jiang and Pugh, 2009) and trimeric Hsf1 binding motif locations for TFIID and (B) SAGA dominated promoters with the average shown using $10 \mathrm{bp}$ bins in (C) and (D). (E) and (F) Scatterplots for the amount of bound Hsf1 on each of the TFIID (E) and SAGA (F) dominated Hsf1 targets under standard growth conditions versus the drop in mRNA synthesis rates 30 minutes after induction of Hsf1 nuclear depletion. To quantify the correspondence between the binding and expression changes, $r$ - and $p$-values were calculated using the function "cor.test" in the statistical language R. Table EV1 contains the underlying values.

of $4 \mathrm{tU}$ label incorporation, a slow nuclear depletion experiment was performed. This enables measurement of mRNA synthesis rates at multiple Hsf1 levels. Slow depletion was achieved by adding a fifty-fold lower concentration of the inducing agent, resulting in a delayed impact on growth compared to the high concentration (Fig 3A). Monitoring the slow depletion using fluorescence microscopy shows that it takes approximately 90 minutes to deplete Hsf1 from the nucleus (Fig 3B). Promoterbound Hsf1 was monitored during the time-course (examples in Fig 3C and 3D) and the corresponding promoter output was measured by $4 \mathrm{t} \mathrm{U}$ labelling at multiple time-points (Fig 3E). Hsf1 binding and the promoter output both show a gradual decrease, corresponding to a slower depletion of Hsf1 from the nucleus. When the Hsf1 binding and mRNA synthesis data are combined, a clear contrast between the two promoter classes is revealed (Fig 4). Fig 4A depicts for each Hsf1 target, the fold 
A

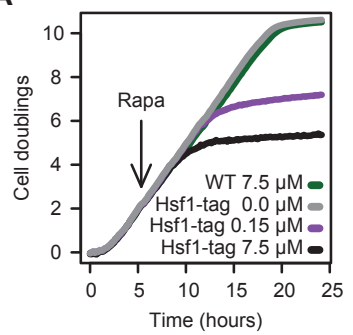

C

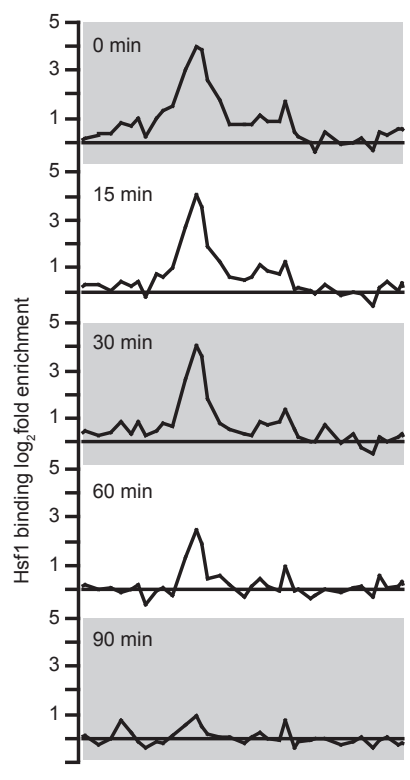

Probes

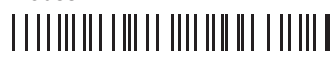

Chr VII

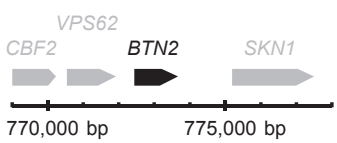

B
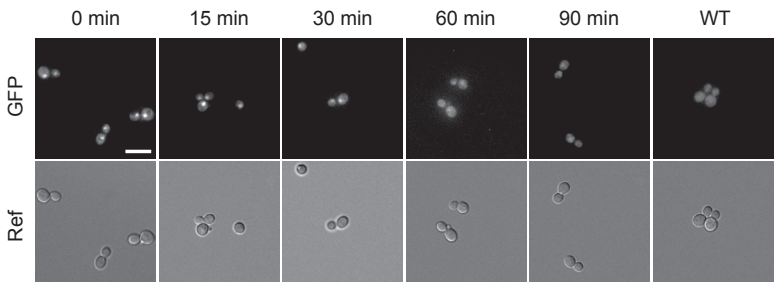

E

D
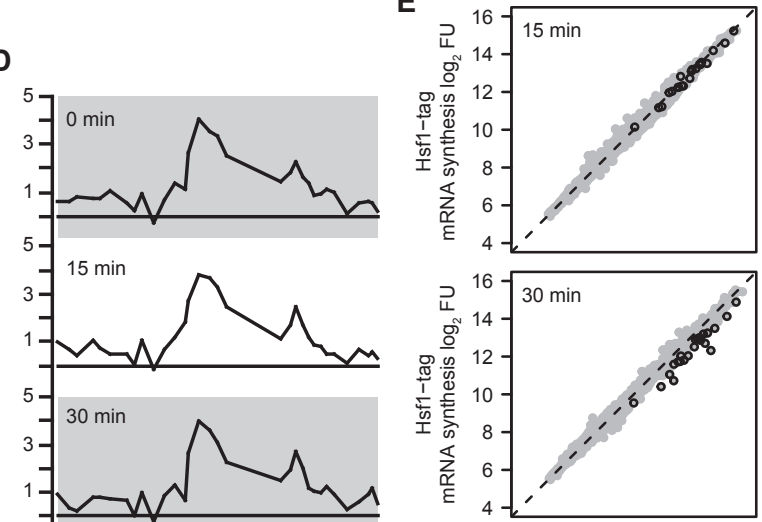

$60 \mathrm{~min}$
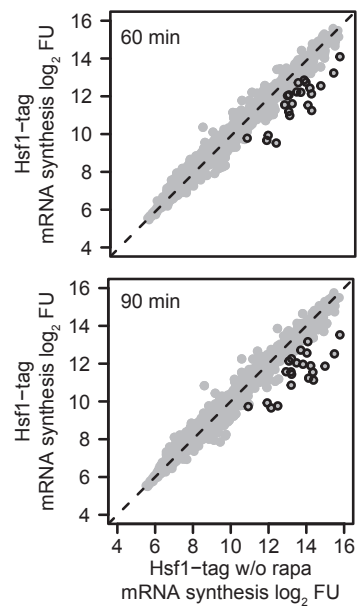

Figure 3. Slow nuclear depletion of Hsf1 using a lower concentration of rapamycin

(A) Growth curve of Hsf1 tagged strain after exposure to a 50-fold reduced rapamycin concentration, compared to untreated, and to WT and the tagged strain with the high dose. (B) Fluorescence microscopy images of Hsf1 depletion at low rapamycin concentration. Scale bar: $10 \mu \mathrm{m}$. (C) Slow depletion of Hsf1 from BTN2 and (D) SSA1 promoters as measured by high density tiling arrays, using four independent replicates. (E) 4tU mRNA synthesis changes upon slow depletion of Hsf1 from the nucleus. The $\mathrm{x}$ - and $y$-axes plot the $\log _{2}$ fluorescent dye intensities of the probes representing each gene (dots), averaged over four replicates, during the time course (y-axis) versus the tagged strain before depletion (x-axis). Circled dots indicate direct Hsf1 targets. 
A

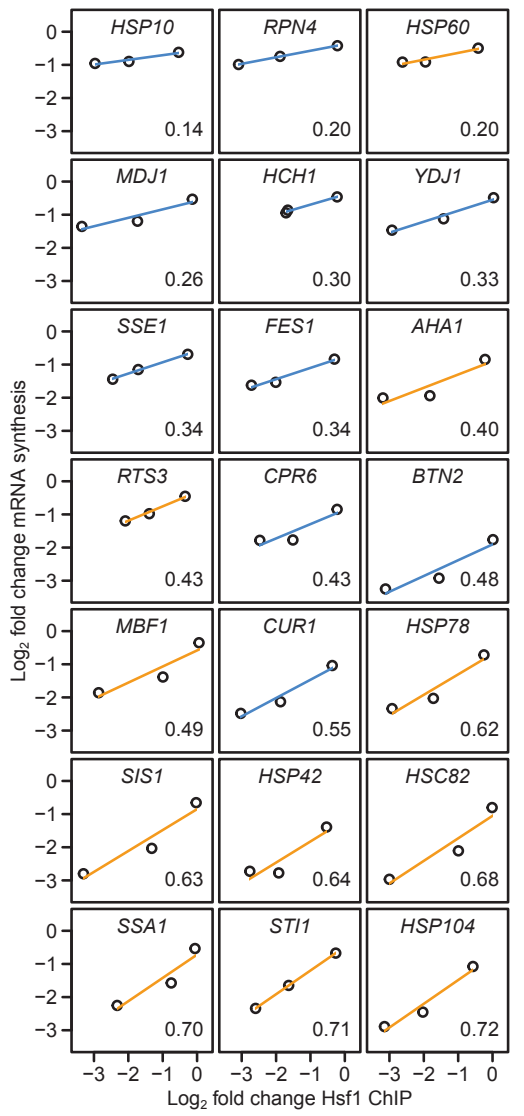

B

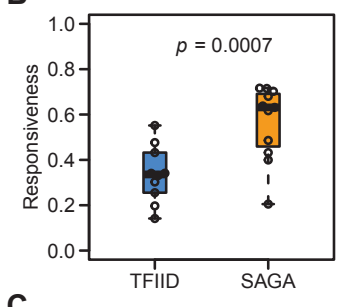

D
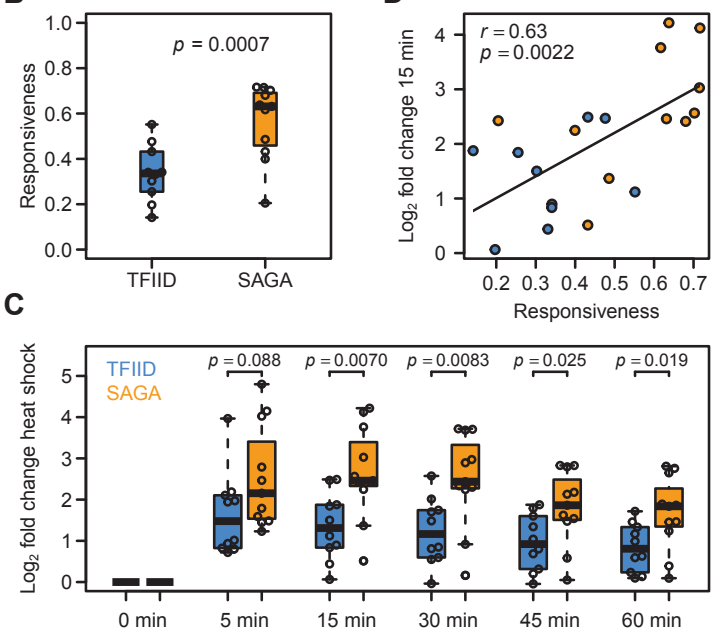

Figure 4. SAGA dominated Hsf1 targets are more responsive than TFIID dominated Hsf1 targets (A) Responsiveness (measured as the slope of the line fit to Hsf1 binding change versus mRNA synthesis change) of Hsf1 targets ordered from low to high. Blue lines are TFIID regulated genes and orange lines are SAGA regulated genes. The responsiveness of each target is shown in the bottom right corner. (B) Boxplot of responsiveness values shown in (A). The $p$-value was calculated using a linear model with the $\log _{2}$ binding ratio as a continuous covariate (C) $\log _{2}$ fold expression changes of the TFIID dominated (blue) and SAGA dominated (orange) Hsf1 targets in response to heatshock. To determine the significance of difference in upregulation between the two promoter classes, $p$-values were calculated using a twosided t-test. Each time point was grown as biological replicate and measured in technical replicate, yielding four measurements per gene. (D) Expression changes upon 15 minutes of heat shock versus the responsiveness of the direct Hsf1 targets. Colors are the same as in (A). Heat shock expression data was taken from (O'Duibhir et al, 2014). $r$ - and $p$-values were calculated using the function "cor.test" in the statistical language R. Supplemental Fig S1 encompasses additional analyses of responsiveness.

change in mRNA synthesis rates ( $\log _{2} \mathrm{FC}, \mathrm{y}$-axis) versus the fold change in Hsf1 binding ( $\log _{2}$ FC, x-axis). The slope of the line fitted through these data points is a measure for the response in promoter output to a change in the levels of bound Hsf1: promoter responsiveness. Determining responsiveness in this manner yields a value that is independent of the starting amounts of either promoter bound Hsf1 or of initial mRNA synthesis rates. Comparing the responsiveness between the two classes (Fig 4B) shows that the SAGA dominated genes have a higher responsiveness than the 
TFIID dominated promoters. The difference between TFIID and SAGA dominated promoters is also reflected upon ranking the responsiveness (Fig 4A): almost all TFIID dominated promoters (blue) have a lower responsiveness than the SAGA dominated promoters (orange). This agrees with the differences observed in the fast depletion experiment (Fig 2E and 2F), extending the observation to promoters individually. Given the use of an identical activator by the two promoter classes, these experiments demonstrate that responsiveness is inherent to promoter type.

\section{SAGA dominated Hsf1 targets are also more responsive during heatshock}

Before setting out to determine the molecular mechanism that underlies differential responsiveness, it was first determined whether the differential responsiveness observed between SAGA and TFIID Hsf1 targets in the depletion experiments (Fig 2E, 2F, 4A and 4B) is also reflected during physiological changes. Hsf1 is an activator of the heat shock response (Morano et al, 2012), activating its targets when cells are exposed to heat stress. Based on published heat shock gene expression data (O'Duibhir et al, 2014), the TFIID and SAGA dominated Hsf1 targets indeed behave differently upon heat stress, with the SAGA dominated Hsf1 targets showing a larger expression increase compared to the TFIID dominated Hsf1 targets (Fig 4C). This agrees well with the higher responsiveness of the SAGA dominated targets observed upon Hsf1 depletion under non heat-shock conditions (Fig 4A and 4B). There is also good correspondence between the responsiveness determined by nuclear depletion of Hsf1 and the heat shock response when analyzed individually (Fig 4D). This is especially striking when bearing in mind that a physiological heat shock involves many more cellular changes besides upregulation of heat shock genes (Morano et al, 2012) and that the heat shock response was monitored without 4tU (O'Duibhir et al, 2014). The concordance (Fig 4D) shows that the higher responsiveness measured for SAGA dominated Hsf1 targets by nuclear depletion is physiologically relevant and reflected by the differential behavior of these targets upon heat shock.

\section{Molecular mechanisms of activator responsiveness}

Having established that SAGA and TFIID promoters are differentially responsive to the presence of an identical activator (Fig 4), we next investigated the molecular mechanisms underlying the difference in responsiveness. First, several trivial explanations were explored. Importantly, the rate of Hsf1 promoter depletion from the two classes is not measurably different (Supplemental Fig S1A), ruling out differential promoter depletion as a possible explanation. Class differences in initial or final mRNA synthesis rates were also ruled out (Supplemental Fig S1B and S1C). There is also no significant correlation between the initial synthesis rates and the responsiveness for either class (Supplemental Fig S1D and S1E), as expected given the way in which responsiveness is calculated. Also, no significant Hsf1 binding motif differences or differential presence of poly[dA:dT] motifs were found between the two classes. Neither was there a differential enrichment for any of the general regulatory factors (GRFs) Abf1, Rap1 or Reb1 (Kasinathan et al, 2014; Rhee \& Pugh, 2011). This indicates that motif differences or binding of GRFs don't account for the difference in responsiveness observed. 
A

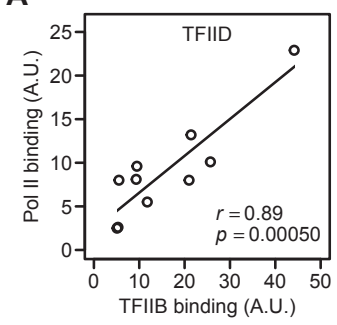

D

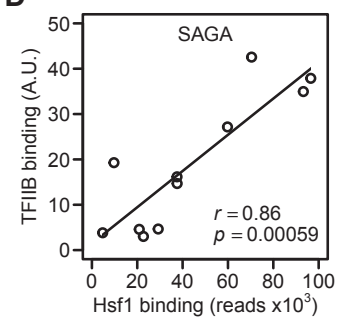

B
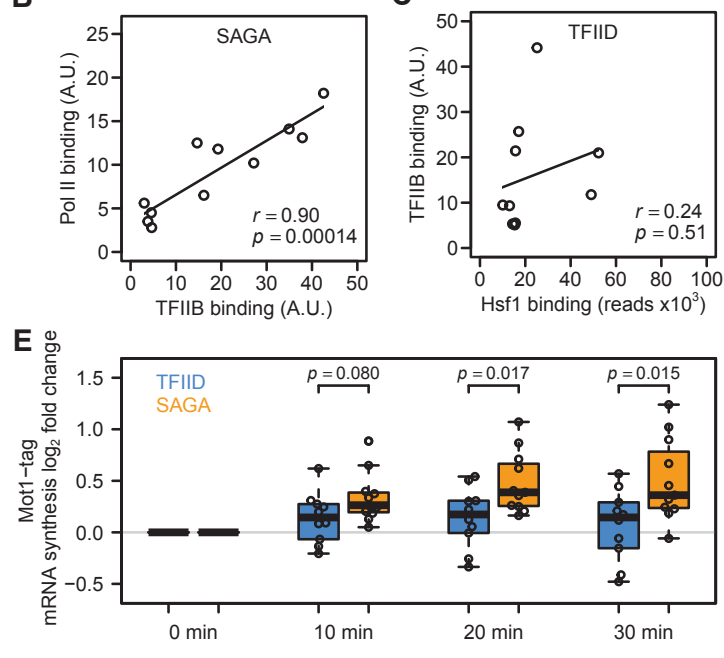

C

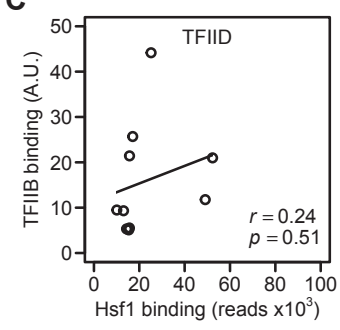

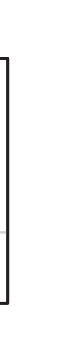

Figure 5. Hsf1 SAGA dominated promoters are sensitive to negative regulation by Mot1.

(A) Correlation between the amount of TFIIB and Pol II bound to the TFIID dominated and (B) SAGA dominated Hsf1 targets. (C) Lack of correlation between the amount of TFIIB binding and the amount of Hsf1 binding for TFIID dominated Hsf1 targets. (D) Correlation between the amount of TFIIB binding and the amount of Hsf1 binding for SAGA dominated targets. TFIIB and Pol II binding data are from Rhee and Pugh, 2012. The $p$-values of (A-D) were obtained as in Figure 2. (E) $\log _{2}$ fold change in $4 \mathrm{tU}$ mRNA synthesis rates for the TFIID (blue) and SAGA (orange) Hsf1 targets immediately following Mot1 nuclear depletion, all compared to the same strain before depletion. The $p$-values were by two-sided t-test. Each time point was grown as biological replicate and measured in technical replicate, yielding four measurements per gene.

Many activators exert a positive influence on transcription through direct recruitment of PIC components (Ptashne \& Gann, 1997; Struhl, 1995). One of the essential components of the PIC is TFIIB, which directly contacts DNA, TBP and RNA polymerase II (Grünberg \& Hahn, 2013; Sainsbury et al, 2015). For both the TFIID and SAGA dominated Hsf1 targets there is a good correlation between TFIIB binding and Pol II presence (Fig 5A and 5B). This suggests that any PIC formation steps following TFIIB binding and that lead to Pol II recruitment proceed similarly for the two promoter classes. Interestingly, this concordance between the two promoter classes is not observed when comparing TFIIB binding and Hsf1 binding. Whereas on SAGA dominated promoters the amount of TFIIB correlates well with the amount of Hsf1, for TFIID promoters this relationship is almost absent (Fig 5C and 5D). This suggests that Hsf1 is important for PIC formation or stability on SAGA promoters, but less so for TFIID dominated genes and that the distinguishing step takes place at or before TFIIB binding.

\section{Negative regulation by Mot1 is associated with higher responsiveness}

The disconnect between Hsf1 binding and TFIIB presence at TFIID dominated promoters suggests that there is a difference in PIC formation or stability at the two promoter classes. A key step in PIC formation is TBP recruitment (Buratowski et al, 1989; Davison et al, 1983; Roeder, 1996), which proceeds differently for the 
two promoter classes due to different preferential coactivator use (Basehoar et al, 2004; Huisinga \& Pugh, 2004; Rhee \& Pugh, 2012). A known regulator of TBP promoter dynamics is Mot1 (Tora \& Timmers, 2010; Viswanathan \& Auble, 2011), which removes TBP preferentially from SAGA dominated/TATA-box containing promoters (Zentner \& Henikoff, 2013). Differential TBP turnover can potentially explain a difference in responsiveness. When TBP is more rapidly removed from one of the classes of promoters, transcription would be more efficiently shut down in this class, leading to a larger response upon depletion of an activator. Indeed, TBP turnover has been shown to be high in SAGA dominated promoters and low in TFIID dominated promoters (van Werven et al, 2009). Furthermore, genes that become derepressed upon Mot1 inactivation are enriched for SAGA dominated genes (Spedale et al, 2012). Since Mot1 evicts TBP from SAGA dependent promoters and given the indications that there is a difference in PIC stability between the two promoter classes (Fig 5C and 5D), it was next tested whether negative regulation by Mot1 underlies the difference in responsiveness.

Mot1 was tagged with FRB-GFP in the S288C background and mRNA synthesis was monitored with $4 \mathrm{tU}$ immediately after induction of Mot1 nuclear depletion (Fig 5E). In this experiment too, SAGA dominated Hsf1 targets behave differently, showing more sensitivity in the form of increased mRNA synthesis rates upon loss of Mot1 function (Fig 5E). This fits very well with the idea that continuous Mot1 mediated TBP removal on SAGA dominated promoters makes such genes more dependent on activator presence and therefore more responsive. As is discussed later, the differential sensitivity of the two promoter classes to Mot1 also explains why TFIID is linked to housekeeping genes and SAGA to regulatable genes.

\section{Nucleosome repositioning also underlies higher responsiveness}

Sensitivity to Mot1 loss of function is significantly different for the two Hsf1dependent promoter classes (Fig 5E), but the difference is modest. This is in part due to an inability to monitor changes after longer periods of Mot1 nuclear depletion because of the onset of indirect effects (O'Duibhir et al, 2014). Nevertheless, mechanisms other than TBP eviction potentially also play a role, including promoter chromatin architecture. Nucleosome configurations generally differ between the two promoter classes (Albert et al, 2007; Cairns, 2009; Rhee \& Pugh, 2012; Tirosh \& Barkai, 2008), see also Fig 2). This is of interest since chromatin can have diverse roles in shaping regulatory properties including responsiveness (Lam et al, 2008; Raveh-Sadka et al, 2009; Tirosh \& Barkai, 2008).

To investigate chromatin in the presence and absence of Hsf1, nucleosome positions were determined genome-wide using micrococcal nuclease followed by sequencing (MNase-seq), both before and 30 minutes after induction of Hsf1 nuclear depletion. Because the sensitivity of nucleosomes to MNase varies (Kubik et al, 2015; Mieczkowski et al, 2016; Weiner et al, 2010; Xi et al, 2011; Henikoff et al, 2011; Kent et al, 2011), chromatin was digested with four different concentrations of MNase. Fig 6 shows nucleosomal DNA midpoints for the average TFIID dominated Hsf1 target (left), either in the presence (grey) or absence of Hsf1 (line), and for the four different 
MNase concentrations ( $0.05-3 \mathrm{U}$, top to bottom). The corresponding average SAGA dominated Hsf1 target is depicted on the right of Fig 6, with three examples of each promoter class depicted in Fig 7. Supplemental Fig S2 contains plots for all 21 individual Hsf1 dependent promoters.

Nucleosome occupancy and positioning on the TFIID dominated targets are only mildly affected by Hsf1 depletion, with only a slight increase in occupancy visible (Fig 6 left), consistent with reduced transcription rates. The high degree of activator independence on TFIID dominated promoters is clear from both the average plot (Fig 6 left), as well as from each individual promoter (Fig 7 left and Supplemental

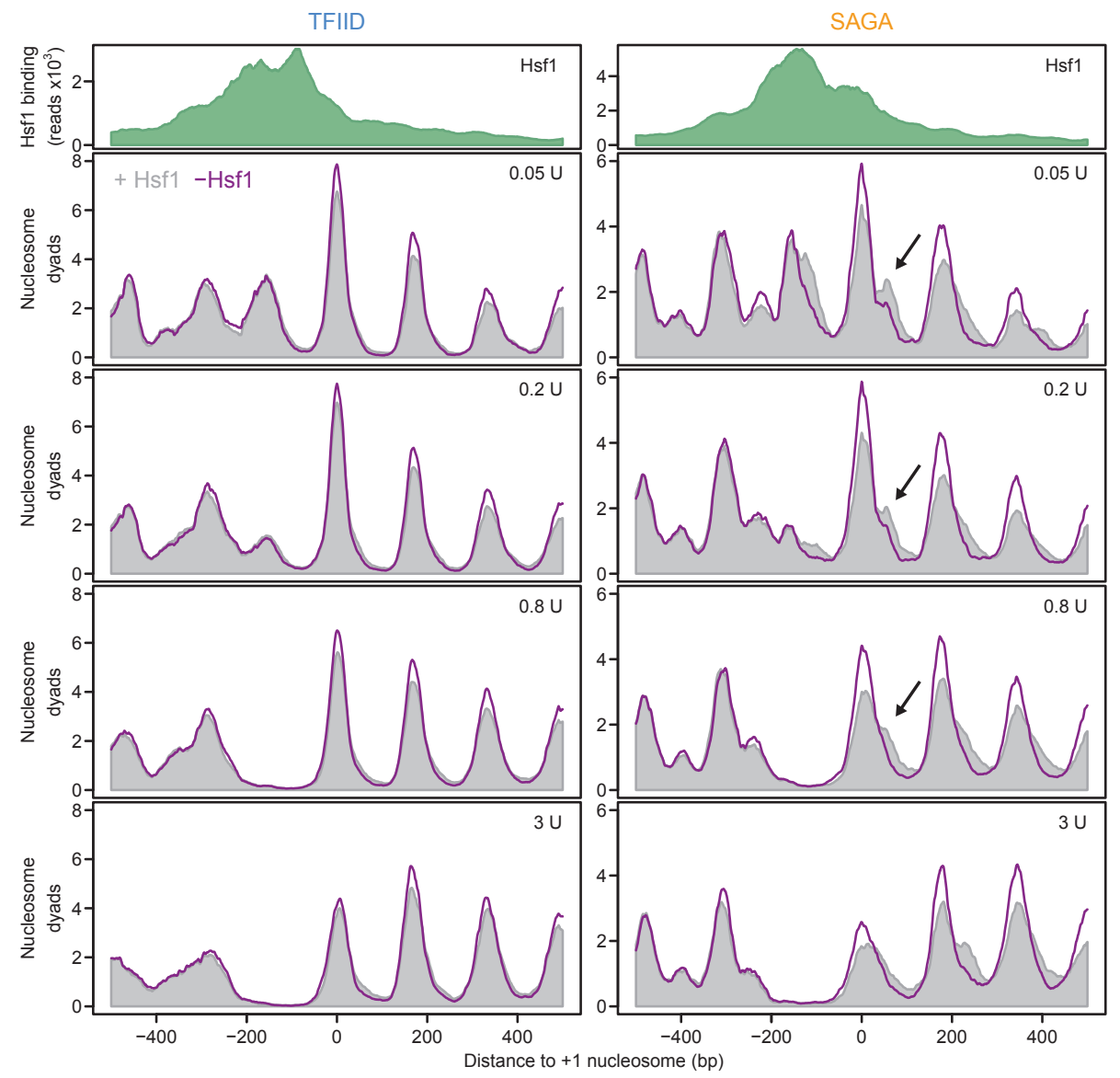

Figure 6. Hsf1 removal results in nucleosomal repositioning on SAGA dominated promoters.

Average profiles of MNase-seq nucleosomal DNA midpoint positions for the TFIID dominated (left) and SAGA dominated (right) Hsf1 targets. The upper panels display the average Hsf1 binding by ChIP-seq (green). The lower panels show nucleosomal DNA midpoints using $31 \mathrm{bp}$ smoothing in the presence (grey) and absence (purple line) of Hsf1 (30 minutes of nuclear depletion). Each profile is the average of three biological replicates each scaled to 10 million mapped reads. The values in the top right corner indicate the amount of MNase used to digest the chromatin. The data is aligned to the +1 nucleosome. The arrows indicate the downstream +1 nucleosome in the SAGA dominated promoters, that shifts upstream upon depletion of Hsf1. 

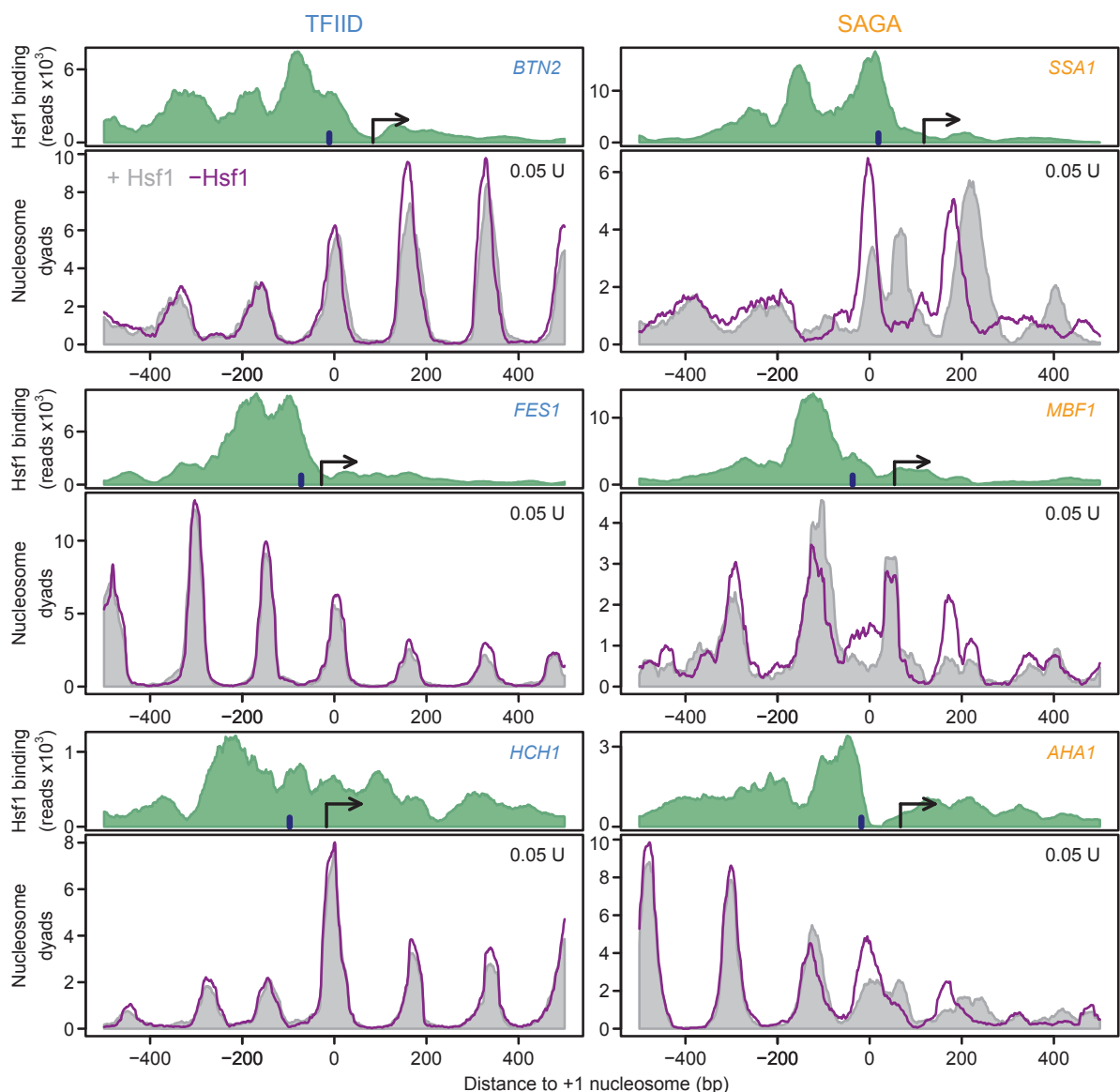

Figure 7. Individual examples of nucleosome repositioning on SAGA dominated promoters

As in Figure 6, but for three individual promoters from each class as indicated by the gene name in each panel. The three genes on the left are TFIID dominated and the three genes on the right are SAGA dominated. The arrow denotes the transcription start site (Nagalakshmi et al., 2008). The dark blue bar indicates the location of the TATA (-like) element (Rhee and Pugh, 2012). The examples shown were from the experiments digested using 0.05 units of MNase. All 21 Hsf1 target promoters are depicted in Supplemental Fig S2.

Fig S2). In contrast, nucleosomes on the SAGA dominated Hsf1 targets are much more strongly affected by Hsf1 (Fig 6 and 7 right). On the SAGA dominated targets, the +1 nucleosome forms two peaks in the presence of Hsf1, whereby the minor, most downstream peak (Fig 6 right, arrow), shifts back upstream upon Hsf1 removal. Due to averaging (Fig 6), some extremely large repositioning differences on individual SAGA dominated promoters are lost (Fig 7 right). On the SSA1 promoter for example, two different cell populations of +1 nucleosome DNA fragment midpoints are observed in the presence of Hsf1, with the most downstream population completely disappearing upon Hsf1 removal (Fig 7 right). This indicates that the +1 nucleosome is in the downstream position when the gene is active, and in the upstream position when the gene is inactive. We infer that the two nucleosome positions represent 
different cell populations because the spacing $(60 \mathrm{bp})$ is too small for simultaneous binding. Upon removal of Hsf1, nucleosomes shift from the downstream (active) position to the upstream (inactive) position. Most of the SAGA dominated Hsf1 targets show similar effects, although to a lesser extent (Fig 7 and Supplemental Fig S2). This is in stark contrast to the TFIID dominated promoters, only one of which shows slight nucleosome repositioning: CUR1, the most responsive TFIID dominated promoter (Supplemental Fig S2).

The results show that Hsf1 removal has different consequences at the two promoter classes. For the TFIID dominated targets, Hsf1 removal has little immediate effect on nucleosome positioning. On SAGA dominated genes, Hsf1 removal results in repositioning of the +1 nucleosome upstream into what is apparently a transcriptionally less favourable position. These results agree with the idea that transcriptional plasticity is linked to nucleosome configuration (Lam et al, 2008; Raveh-Sadka et al, 2009; Tirosh \& Barkai, 2008), as well as with the idea that an appropriately positioned +1 nucleosome is important for transcription (Rhee \& Pugh, 2012). Notably, Fig 6 shows that removal of an activator results in nucleosomal repositioning on SAGA dominated targets, but not on TFIID dominated targets, even though genes from both classes depend on the same activator for promoter output. Besides differential TBP turnover, differences in nucleosome configuration therefore likely also contribute to the different responsiveness found between TFIID housekeeping and SAGA regulatable genes.

\section{Discussion}

The central question addressed here is regulatability of transcription and what dictates this property. The two different core promoter types, TATA-box/SAGA dominated and TATA-like/TFIID dominated, have in the past been associated with increased regulatability and housekeeping transcription respectively (Basehoar et al, 2004; Huisinga \& Pugh, 2004; Tirosh \& Barkai, 2008; Tirosh et al, 2006). This association was based on an enrichment for TATA-box/SAGA dominated promoters in genes with higher expression variation. An important finding of this study is that the property of responsiveness is directly inherent to core promoter type. Genes dependent on a single transcriptional activator show different degrees of responsiveness and this difference in regulatory dynamics is due to core promoter class. This is important to emphasize since previous explanations put forward for the higher variation in gene expression observed for TATA-box/SAGA dominated genes include increased competition between activators and nucleosomes, combined with promoter targeting by a greater number of regulatory factors. Although it cannot be formally excluded that the differences are specific to Hsf1 targets, the analyses presented here indicate that responsiveness is directly associated with core promoter type rather than the number or type of regulators. Evidence for two molecular mechanisms are presented. Both involve dynamic negative regulation that make SAGA/TATA-box promoters more dynamically dependent on an activator. 
Increased turnover of TBP would serve to make a promoter more responsive to activator presence because on such a promoter transcription would otherwise be much more quickly shut down. Both this and the converse SAGA/TATA-box promoter upregulation upon Mot1 inactivation are observed here. The detailed analyses of a single transcription factor regulon fits well with previous genome-wide and biochemical studies showing that Mot1 increases TBP turnover preferentially at SAGA dominated/TATA-box promoters (Spedale et al, 2012; Tora \& Timmers, 2010; Viswanathan \& Auble, 2011; van Werven et al, 2009; Zentner \& Henikoff, 2013). This mechanism also explains the long-standing observation that TATA-box type is associated with regulatability properties (Struhl, 1986). Firstly, a consensus TATA-box puts more strain on DNA which may contribute to Mot1-mediated TBP release (Tora \& Timmers, 2010). Flanking DNA sequences likely also play a role (Viswanathan et al, 2016). In addition, structural analyses indicates that TBP is protected from Mot1 interaction in the context of TFIID, but not in the context of SAGA (Anandapadamanaban et al, 2013; Bagby et al, 2000; Ravarani et al, 2016; Wollmann et al, 2011). This therefore also contributes to our understanding of why TFIID is associated with housekeeping genes, as TBP in the TFIID complex, bound to a consensus TATA-box, is less susceptible to removal.

TBP turnover is unlikely to be the only mechanism contributing to promoter responsiveness. The differential effects on nucleosome repositioning between TFIID/TATA-like and SAGA/TATA-box promoters upon activator loss also implicates differences in nucleosome configuration. The most straightforward interpretation is that the activator Hsf1 is important for keeping SAGA/TATA-box promoters in a nucleosomal configuration that is amenable to transcription. Upon Hsf1 loss, nucleosome positioning reverts to a configuration that is less favourable for transcription. In this sense the mechanism is similar to increased TBP turnover, i.e. increased responsiveness due to more dynamic state transformations of SAGA/ TATA-box genes. A correctly positioned +1 nucleosome is thought to be important for transcription (Rhee \& Pugh, 2012) and previous studies have indeed shown differences in nucleosome organisation between SAGA/TATA-box and TFIID/ TATA-like promoters that includes the +1 nucleosome (Albert et al, 2007; Cairns, 2009; Jiang \& Pugh, 2009; Rhee \& Pugh, 2012; Tirosh \& Barkai, 2008) . Models implicating nucleosomes in regulatability (Lam et al, 2008; Raveh-Sadka et al, 2009; Tirosh \& Barkai, 2008) often apply direct competition as mechanism. The position of Hsf1 binding on the SAGA dominated Hsf1 targets is only compatible with a direct +1 nucleosome competition model in a few cases (Supplemental Fig S2) and recruitment of nucleosome remodelling activity by Hsf1 may therefore be involved (Erkina et al, 2010; Shivaswamy \& Iyer, 2008).

The findings presented here fit with recent studies showing the importance of an appropriate interplay between transcriptional regulators and chromatin architecture during gene expression (Kubik et al, 2015; Reja et al, 2015; Nocetti \& Whitehouse, 2016). As in other studies, here too SAGA dominated promoters show more changes in nucleosome positioning compared to TFIID dominated promoters. It has been reported that generally, TATA-less genes have regions of lower bendability in their 
promoters (Tirosh et al, 2007). This disfavours nucleosome binding and stimulates a more inherently open nucleosome configuration. Lack of such regions may cause SAGA dominated genes to be more dependent on nucleosome repositioning through activator-dependent recruitment of remodelers.

Responsiveness is correlated to gene expression noise and may be mechanistically linked (Lehner, 2010). This agrees with the higher responsiveness of SAGA/TATAbox promoters since the presence of a TATA-box increases gene expression noise (Murphy et al, 2010; Raser \& O'Shea, 2004). Related to this, the TATA-box has a major role in determining transcriptional burst size which may in fact reflect responsiveness (Hornung et al, 2012). The latter study also highlights that individual promoter features are not always unambiguously associated with promoter properties, an aspect also illustrated here. For example HSP60 is distinct from other SAGA/TATAbox promoters since it is less responsive than most of the TFIID/TATA-less promoters analyzed (Fig 4A), indicating that there is still much to be learnt regarding gene regulation rules. In line with this, it is important to point out that TFIID does not function exclusively at TATA-like promoters (Huisinga \& Pugh, 2004; Basehoar et al, 2004; Rhee \& Pugh, 2012). Within the 21 Hsf1 targets defined here there are two TFIID dominated genes with a TATA-box and there are two SAGA dominated genes that lack a TATA-box. This exemplifies that the difference between SAGA and TFIID dominated genes is not black-and-white and also explains why some TFIID dominated genes show increased expression upon depletion of Mot1 (Fig 5E). Combinatorial control is a well-established concept in regulation of gene expression that refers to multiple transcription factors acting in synergistic combinations to specify unique gene expression patterns. This study adds an additional facet to combinatorial control mechanisms: the action of an identical activator can have a different outcome depending on promoter context.

\section{Materials and Methods}

\section{Strain creation}

The anchor away system (Haruki et al, 2008) was recreated in the Saccharomyces cerevisiae S288C/BY4742 strain. To achieve this FPR1 was deleted, a tor1-1 mutation was introduced to desensitize the strain to rapamycin and RPL13A was tagged with $2 x F K B P 12$ as in the original anchor away system (Haruki et al, 2008). This strain was subsequently used here as the parental strain to create each specific anchor away strain. This strain is referred to as wildtype (WT) throughout the manuscript. In order to create the specific anchor away strains, Hsf1 was tagged with an FRB-yEGFP tag and Mot1 with an FRB-yEGFP-3V5 tag. Proper tagging was checked using PCR, western blotting and microscopy.

\section{Growth curves}

Strains were streaked from $-80^{\circ} \mathrm{C}$ stocks onto appropriate selection plates and grown for 3 days. Liquid cultures were inoculated with independent colonies and grown overnight $(\mathrm{ON})$ in Synthetic Complete (SC) medium: 2 gr/L Drop out mix 
Complete and $6.71 \mathrm{gr} / \mathrm{L}$ Yeast Nitrogen Base without AA, Carbohydrate \& w/AS (YNB) from US Biologicals (Swampscott, USA) with 2\% D-glucose. The following day ON cultures were used to inoculate $1.5 \mathrm{ml}$ of SC media and cells were grown in an Infinite F200 platereader (Tecan) alongside a WT parental strain at $30^{\circ} \mathrm{C}$. Starting OD595 of cultures was $0.15+/-0.05$ and the OD595 was measured every 10 minutes. Cells were diluted 1:3 after two doublings $(\mathrm{OD}=0.60)$ by removing $1 \mathrm{ml}$ of medium and adding $1 \mathrm{ml}$ of fresh SC medium containing $2 \%$ glucose and various concentrations of rapamycin. This dilution procedure was repeated several times. These measurements were used to calculate doubling times and to determine the rapamycin concentration to use for slow depletion experiments.

\section{Anchor away depletion}

Hsf1 and Mot1 were depleted from the nucleus by adding rapamycin, dissolved in DMSO, to a final concentration of $7.5 \mu \mathrm{M}$. To achieve a slow depletion, a 50x lower concentration of rapamycin was used (final concentration $0.15 \mu \mathrm{M}$ ). For the " $t=0$ " samples no rapamycin, but the same volume of DMSO was added.

\section{Microscopy}

Nuclear Hsf1 depletion rates were determined in exponentially growing cells by fluorescence microscopy. A non-tagged parental strain was used as a negative control. All the images were set to have identical min and max brightness values. Circles were manually drawn around cells to measure pixel intensities and the skewness of these intensities was calculated using the ImageJ tool. Nuclear GFP intensity was quantified for every cell at each time point as the skewness of the GFP signal (pixel intensities) +1.5 in each cell.

\section{RNA labelling and extraction}

4-thiouracil $(4 \mathrm{tU})$ was added to cell cultures at a final concentration of $5 \mathrm{mM}, 6$ minutes prior to mRNA extraction. Cells were then harvested by centrifugation (3952 x g for 3 minutes) and pellets were immediately frozen in liquid nitrogen after removal of supernatant. Frozen cells $\left(-80^{\circ} \mathrm{C}\right)$ were resuspended in $500 \mu \mathrm{l}$ Acid Phenol Chloroform (Sigma, 5:1, pH 4.7). Immediately an equal volume of TESbuffer (TES: $10 \mathrm{mM}$ Tris pH 7.5, $10 \mathrm{mM}$ EDTA, 0.5\% SDS) was added. Samples were vortexed hard for 20 seconds and incubated in a water bath for 10 minutes at $65^{\circ} \mathrm{C}$ and vortexed again. Samples were then put in a thermomixer $\left(65^{\circ} \mathrm{C}, 1400\right.$ rpm) for 50 minutes. Samples were spun for 20 minutes in an Eppendorf bench top centrifuge at $14000 \mathrm{rpm}$ at $4^{\circ} \mathrm{C}$. Phenol extraction was repeated once, followed by a Chloroform:Isoamyl-alcohol (25:1) extraction. RNA was precipitated with Sodium Acetate (NaAc 3M, pH 5.2) and ethanol $\left(96 \%,-20^{\circ} \mathrm{C}\right)$. The pellet was washed with ethanol and dissolved in sterile water (MQ) to $1 \mu \mathrm{g} / \mu \mathrm{l}$ final concentration. RNA samples were heated for $10 \mathrm{~min}$ at $60^{\circ} \mathrm{C}$, then immediately put on ice for $2 \mathrm{~min}$. 100 $\mu \mathrm{g}$ RNA was then biotinylated with $200 \mu \mathrm{l}$ of $1 \mathrm{mg} / \mathrm{ml}$ biotin-HDPD and unbound biotin was removed using chloroform extraction. Labeled RNA was separated from total RNA on a $\mu \mathrm{MACs}$ column containing streptavidin conjugated magnetic beads. The bound RNA was washed $6 x$ with $65^{\circ} \mathrm{C}$ washing buffer $(100 \mathrm{mM}$ Tris $\mathrm{pH} 7.5,10$ $\mathrm{mM}$ EDTA, $1 \mathrm{M} \mathrm{NaCl}, 0.1 \%$ Tween20) and eluted using $200 \mu \mathrm{l}$ of $100 \mathrm{mM}$ DTT. The 
RNA was then purified with an RNeasy MinElute Cleanup Kit from Qiagen.

\section{Microarray profiling}

Dual-channel 70-mer oligonucleotide arrays were employed with a $4 \mathrm{tU}$ common reference WT RNA. For most microarray experiments, each measurement point is the average of two biological replicates, each profiled as a technical replicate in dye-swap, yielding four replicates that were averaged and statistically analyzed by limma (Smyth et al, 2005) versus either wild type or the same strain at $\mathrm{t}=0$. For the fast nuclear depletion of Hsf1 one biological replicate was used. Apart from $4 \mathrm{tU}$ labelling and mRNA extraction all procedures were identical and are described in detail in (Kemmeren et al, 2014). Calculations were done using the statistical language $\mathrm{R}$ version 3.0.1 on a Linux machine running CentOS 5.5. Expression changes are the $\log _{2}$-ratios relative to the median of the four wild types at the same time point, or of the same strain at $\mathrm{t}=0$.

\section{Chromatin Immunoprecipitation (ChIP)}

ChIP was carried out as previously described (van Bakel et al, 2008) with some modifications. In short, $250 \mathrm{~mL}$ of mid-log growing yeast cells $\left(\mathrm{OD}_{595}=1.0\right)$ were crosslinked with $2 \%$ formaldehyde for $30 \mathrm{~min}$ at $30^{\circ} \mathrm{C}$, the reaction was quenched with glycine (final concentration $=125 \mathrm{mM}$ ), and cells were collected by centrifugation. Rapamycin addition was staggered during the timecourse experiment to allow harvesting of all samples at the same time and OD. Subsequently, cells were spheroplasted according to the protocol of the Rando lab (Rando, 2010) and then directly sonicated (Bioruptor, Diagenode: ten cycles, $30 \mathrm{sec}$ on/off, medium setting. $200 \mu \mathrm{L}$ chromatin extract was incubated with $10 \mu \mathrm{L}$ of anti-GFP antiserum (3h, RT) which had been coupled to Protein G agarose beads (Roche 11243233 01) overnight at $4^{\circ} \mathrm{C}$. After incubation with the antibody, the beads were washed twice in FA lysis buffer (50 mM HEPES KOH pH 7.5, 150 mM NaCl, 1 mM EDTA, 1\% Triton X-100, $0.1 \%$ Na-deoxycholate, $0.1 \%$ SDS), twice with FA lysis buffer containing $0.5 \mathrm{M} \mathrm{NaCl}$, and twice with $10 \mathrm{mM}$ Tris at $\mathrm{pH} 8.0,0.25 \mathrm{mM} \mathrm{LiCl}, 1 \mathrm{mM}$ EDTA, 0.5\% Nonidet P-40 and $0.5 \%$ Na-deoxycholate. Cross-links of the ChIP samples were reversed overnight at $65^{\circ} \mathrm{C}$ in $150 \mu \mathrm{L} 10 \mathrm{mM}$ Tris- $\mathrm{HCl}(\mathrm{pH} 8.0), 1 \mathrm{mM}$ EDTA, 1\% SDS. Samples were treated with RNAse and proteinase K, and DNA was recovered for further analysis using phenol extraction.

\section{ChIP-seq and peak finding}

Prior to library preparation the samples were sub-sheared to obtain fragments in the optimal size range (Mokry et al, 2010). Both ChIP and input samples were sequenced single end $(50 \mathrm{bp})$ using a SOLiD Wildfire platform. The reads were aligned to the sacCer3 genome assembly (February 2011) using bwa (Li \& Durbin, 2009) with the settings ' $-\mathrm{c}-125-\mathrm{k} 2-\mathrm{n} 10$ ', yielding 32.5 million mapped reads (48\%) for the ChIP sample, and 48.1 million mapped reads (60\%) for the input sample. Hsf1-binding peaks were detected using the program CisGenome (Ji et al, 2008). The maximal $\log _{2}$ (fold change) between IP and WT DNA fragment counts ( $\max \log _{2}$ (fold change)), peak width and the normalized number of reads in the peak were used for further analysis. 


\section{ChIP-qPCR}

DNA samples were recovered after ChIP as described above. The fold change of two Hsf1 targets (BTN2 and SSA1) over two control regions (HMR and POL1) was measured by qPCR, which were performed in 384 well plates using $6 \mu$ IQ SYBR Green super mix (BioRad), $2 \mu$ l of each primer set and $2 \mu \mathrm{l}$ of DNA. The total volume per reaction was $10 \mu \mathrm{l}$. To create a standard curve $10 \mu \mathrm{l}$ of each of the input samples were combined and used undiluted and diluted at concentrations of 1:10, 1:100 and 1:1000. The qPCRs were run on a 7900HT fast real time PCR machine (Applied Biosystems). IP and mock samples were first normalized to their corresponding input samples. The fold change of BTN2 and SSA1 were then calculated by dividing the signal from these genes by the average signal of the two negative control genes (HMR and POL1). All samples were measured as biological triplicates and technical quadruplicates. All qPCR primers are shown in Table S2.

\section{ChIP-chip timecourse}

For the slow depletion time course cells were grown for almost two doublings (from $\mathrm{OD}=0.15$ to $\mathrm{OD}=0.5$ ). Subsequently rapamycin was added to a final concentration of $0.15 \mu \mathrm{M}$. Additions were staggered so that all the timepoints (WT, t0, t15, t30, t60, t90) were ready at the same OD and time. For the t0, no rapamycin but a similar volume of DMSO was added for 30 minutes. ChIP-chip hybridizations and normalizations were done as described (van Bakel et al, 2008; van Werven et al, 2009). Four independently grown cultures were used for each time point, two of which were done in dye-swap. To control for non-specific antibody binding, the WT track was subtracted from all other tracks. Peaks were found by searching for genomic locations where two adjacent probes had at least a fold change of 2 , with a $p$-value lower than 0.05 . For each peak the probe with the highest fold change was taken and subsequently followed during the time course. If two adjacent probes switched between being the biggest fold changer within the peak during the time course, the average of the fold change and $p$-value of these two probes were taken for subsequent analysis. The depletion rate was calculated by plotting the $\log _{2}$ fold change in Hsf1 binding against time and fitting a line through these points. The slope of this line was used as the depletion rate from the promoters.

\section{Motifs}

To find de novo motifs in the $1000 \mathrm{bp}$ upstream promoter regions of the Hsf1 targets genes, the MEME tool was used (Bailey et al, 2009). The default settings were used except for minimum motif size, which was set to 5 , minimum width to 5 and the distributions of motifs was set to "any number of repetitions". Sites were identified based on 57 high confidence Hsf1 bound targets. These targets were bound in both the ChIP-chip and ChIP-seq experiments.

\section{Mapping of binding sites}

The RSAT tool, (Turatsinze et al, 2008), was used to map significant ( $p$-value $<0.001$ ) presence of a found motif to the promoter regions of the Hsf1 targets. As a motif-input fasta sequences files were given that were found by MEME and used by MEME to create the motif. The standard settings were used except for the background, which 
was set to Saccharomyces cerevisiae upstream regions without ORFs. For the scanning options sequence origin was set to 'start', return to 'site + pval' and $p$-value was set to 0.001 . The identified motifs were mapped to $650 \mathrm{bp}$ promoters of the function Hsf1 targets. In parallel, published nucleosome positions (Jiang \& Pugh, 2009) were also mapped. The average presence of the motif and published nucleosomes was calculated for the SAGA and TFIID dominated genes and shown in 10bp bins.

\section{Direct Hsf1 targets}

The direct Hsf1 targets are a subset of the genes bound both in the ChIP-seq and the ChIP-chip experiments (57 targets), that also robustly change in expression (FC $>1.7, p<0.01$ ) in both the fast and the slow Hsf1 depletion experiments (22 targets). HSP82 is omitted from all analysis (except the motif discovery) due to transcript cross-hybridization with its ortholog HSC82. As HSC82 is far more highly expressed, HSP82 is considered to have a negligible effect on the measured mRNA levels of HSC82 (Borkovich et al, 1989). Therefore HSC82 is included in the analysis, resulting in 21 direct targets.

\section{Responsiveness}

To calculate the responsiveness, the ChIP-chip and $4 \mathrm{tU}$ slow depletion time course data of the direct Hsf1 targets was used. The $\log _{2}$ (fold change) values of the ChIPchip time course were plotted against the $\log _{2}$ (fold change) values of the $4 \mathrm{tU}$ labeling time course, in each case compared to the corresponding $t=0$ sample. A line was fitted through the last three time points $t 30, t 60$ and $t 90$. The slope of this line was used as a measure for responsiveness. The significance of the slope difference in the mRNA expression versus binding plots was calculated using the linear model

$$
\mathrm{E}=\mathrm{G}+\mathrm{C}+\mathrm{B}+\mathrm{CB}+\varepsilon
$$

with $\mathrm{E}$ the $\log _{2}$ (fold change) in mRNA expression; $\mathrm{G}$ the effect of the gene; $\mathrm{C}$ the effect of the gene class, a dichotomous variable that is either SAGA or TFIID; B the $\log _{2}$ (binding ratio), which is a continuous covariate; $C B$ the interaction between terms $C$ and $B$; and lastly the residual error term $\varepsilon$. The overall fit is 0.88 (adjusted R-squared; $p=1.15 \mathrm{e}-15)$. The only term of interest in the model is the interaction term CB. Its effect is equal to the average difference in slope between the two classes of genes; its $p$-value is 0.00066 . Residuals are normally distributed ( $p=0.28$, Shapiro test) and show no dependency on the independent or fitted values. A simple t-test of the difference, per gene class, in the slopes of their regression lines yields a $p$-value of 0.002 . However, this approach is incorrect as it ignores the variance structure of underlying data.

\section{TFIIB and Pol II occupancy}

For the comparison of TFIIB with Polymerase II and Hsf1 binding, genome wide ChIP-exo data of PIC components was used (Rhee \& Pugh, 2012). TFIIB and Pol II occupancy as provided in "Rhee_SuppData1" were taken and scaled down for visualization purposes (all occupancy values were divided by 100 and 10 for TFIIB and Pol II respectively). 


\section{Heatshock}

$\log _{2}$ expression values of previously published expression data of heat shocked BY4742 cells were used (O'Duibhir et al, 2014). The difference in expression changes between SAGA and TFIID dominated Hsf1 targets were calculated using a twotailed t-test.

\section{Correlations}

All correlations and corresponding $p$-values were calculated using the function "cor.test" of the statistical language $R$, which uses the fact that $\sqrt{ }\left((n-2)^{*} \mathrm{r} 2 /(1-\mathrm{r} 2)\right)$ is $\mathrm{t}$-distributed with (n-2) degrees of freedom.

\section{Micrococcal nuclease chromatin digestion}

Isolation of mono-nucleosomal DNA was done essentially as described (Kubik et al, 2015) with some modifications. In short, $70 \mathrm{ml}$ of yeast cells were grown in $\mathrm{SC}$ at $30^{\circ} \mathrm{C}$ to an OD595 of 1.0 in three independent biological replicates. The cells were crosslinked at RT for 5 minutes using formaldehyde at a final concentration of $1 \%$ and quenched for 5 minutes with glycine (final concentration $=125 \mathrm{mM}$ ). Subsequently the cells were spun down and washed once with sorbitol $(1 \mathrm{M})$ and spheroblasted for 8 minutes using $1 \mathrm{ml}$ of spheroblasting buffer (1 M Sorbitol, $1 \mathrm{mM}$ $\beta$-mercaptoethanol, $10 \mathrm{mg} / \mathrm{ml}$ Zymolyase 100T (USB)). The spheroblasts were spun down and washed twice before resuspending them in $1 \mathrm{ml}$ of MNase digestion buffer (1 M Sorbitol, $50 \mathrm{mM} \mathrm{NaCl}, 10 \mathrm{mM}$ Tris $\mathrm{pH}=7.5,5 \mathrm{mM} \mathrm{MgCl} 2,1 \mathrm{mM} \mathrm{CaCl} 2,0.075 \%$ Nonidet P-40, $1 \mathrm{mM} \beta$-mercaptoethanol, $0.5 \mathrm{mM}$ Spermidine). The samples were divided over $4 \mathrm{x}$ four-fold different MNase amounts (Sigma-Aldrich): 0.046875U, $0.1875 \mathrm{U}, 0.75 \mathrm{U}$ and $3.0 \mathrm{U}$ and incubated at $37^{\circ} \mathrm{C}$ for 45 minutes. The digestion was stopped by putting the samples on ice and immediately adding $15 \mu \mathrm{l}$ of 0.5M EDTA and subsequently $15 \mu \mathrm{l}$ of $10 \%$ SDS. The proteins were degraded by adding $15 \mu \mathrm{l}$ of $(10 \mathrm{mg} / \mathrm{ml})$ proteinase $\mathrm{K}$ and incubating the samples for 1 hour at $37^{\circ} \mathrm{C}$. The crosslinks were reversed by putting the samples at $65^{\circ} \mathrm{C}$ overnight. The next day nucleosomal DNA was isolated using phenol extraction and RNA was digested using RNase A/ T1 (Thermo Scientific; final concentration $0.2 \mathrm{mg} / \mathrm{ml}$ and $250 \mathrm{U} / \mathrm{ml}$ ). The extent of digestion was evaluated on a bioanalyzer 2100 high sensitivity chip.

\section{Sequencing and mapping of nucleosomal DNA}

Sequencing libraries were created from the purified nucleosomal DNA using the NextflexTM rapid DNA-Seq kit (Bioo Scientific) using a modified protocol. The libraries were sequenced paired end $(2 \times 75 \mathrm{bp})$ on a NextSeq500 platform. The paired end reads were mapped to the sacCer3 (February 2011) genome assembly using bowtie2 (Langmead \& Salzberg, 2012) (with the settings ' --no-discordant --no-contain --maxins 1980 --trim5 5 --trim3 15 --end-to-end --sensitive'. Only read pairs with an insert size between 95-225 bp were used for subsequent analyses. To compare the occupancy of all samples, they were scaled to 10 million mapped paired-end reads per sample using genomecov from the bedtools2 suite version 2.250 (Quinlan \& Hall, 2010). The middle of each mate pair was used as the position of the nucleosome dyad. The three replicates for each digestion and time point showed only minimal difference in genome-wide occupancy, hence for the rest of 
the analysis these replicates were merged. The nucleosome dyads were smoothed using a $31 \mathrm{bp}$ running average. The $31 \mathrm{bp}$ window was chosen because it provides clearly separated peaks, but keeps positions with a double peak (in other words: a position with two populations of nucleosomes) that are merged into a single peak to a minimum. The Hsf1 targets were manually aligned to the +1 nucleosome, which was defined as the first nucleosome downstream of the nucleosome depleted region (as is visible in the $0.75 \mathrm{U}$ and 3.0U digestion samples). The summit of the most upstream +1 peak in the Hsf1 depleted, 0.1875U MNase digested samples was used as the position of the +1 nucleosome dyad. The script that was used for the centering and smoothing is available on https://github.com/plijnzaad/phtools/blob/master/ ngs/center+smooth.pl. The TSS location in Fig 6 were taken from (Nagalakshmi et al, 2008), however the location of the TSS in the promoter of STI1 was likely wrongly annotated. The TATA locations were taken from (Rhee \& Pugh, 2012) except for AHA1. Here the TATA is annotated as a TATA-like element although there is a TATA-box without mismatches closer to the canonical position, hence this TATAbox is shown

\section{Data availability}

All sequencing and microarray data is available on GEO through the accession number GSE81481.

\section{Author contributions}

Conceptualization, E.O.D., W.d.J. and F.H.; Investigation, E.O.D., W.d.J., D.v.L. and M.G.K. Formal Analysis, E.O.D., W.d.J., and P.L.; Writing, E.O.D., W.d.J. and F.H.; Supervision, P.K. and F.H.; Funding Acquisition, P.K. and F.H.

\section{Acknowledgements}

We would like to thank Slawomir Kubik and David Shore for help with the MNaseseq protocol; Michael Tolstorukov for advice regarding the MNase titration; Michal Levo and Eran Segal for modeling; the Utrecht sequencing facility for sequencing; the Holstege and Kemmeren group members for assistance and discussions. This work was supported by the Netherlands Organisation for Scientific Research (NWO) grants 016108607 (FH), 91106009 (FH), 86411010 (PK) and by the European Research Council (ERC) grant 671174 DynaMech. 


\section{References}

Albert I, Mavrich TN, Tomsho LP, Qi J, Zanton SJ, Schuster SC, Pugh BF (2007) Translational and rotational settings of H2A.Z nucleosomes across the Saccharomyces cerevisiae genome. Nature 446: 572-576

Anandapadamanaban M, Andresen C, Helander S, Ohyama Y, Siponen MI, Lundström P, Kokubo T, Ikura M, Moche M, Sunnerhagen M (2013) High-resolution structure of TBP with TAF1 reveals anchoring patterns in transcriptional regulation. Nat Struct Mol Biol 20: 1008-1014

Bagby S, Mal TK, Liu D, Raddatz E, Nakatani Y, Ikura M (2000) TFIIA-TAF regulatory interplay: NMR evidence for overlapping binding sites on TBP. FEBS Lett 468: 149-154

Bailey TL, Boden M, Buske FA, Frith M, Grant CE, Clementi L, Ren J, Li WW, Noble WS (2009) MEME SUITE: tools for motif discovery and searching. Nucleic Acids Res 37: W202-208

van Bakel H, van Werven FJ, Radonjic M, Brok MO, van Leenen D, Holstege FCP, Timmers HTM (2008) Improved genome-wide localization by ChIP-chip using double-round T7 RNA polymerase-based amplification. Nucleic Acids Res 36: e21

Basehoar AD, Zanton SJ, Pugh BF (2004) Identification and distinct regulation of yeast TATA box-containing genes. Cell 116: 699-709

Borkovich KA, Farrelly FW, Finkelstein DB, Taulien J, Lindquist S (1989) hsp82 is an essential protein that is required in higher concentrations for growth of cells at higher temperatures. Mol Cell Biol 9: 3919-3930

Buratowski S, Hahn S, Guarente L, Sharp PA (1989) Five intermediate complexes in transcription initiation by RNA polymerase II. Cell 56: 549-561

Cairns BR (2009) The logic of chromatin architecture and remodelling at promoters. Nature 461: 193-198

Davison BL, Egly JM, Mulvihill ER, Chambon P (1983) Formation of stable preinitiation complexes between eukaryotic class B transcription factors and promoter sequences. Nature 301: 680-686

Deaton AM, Bird A (2011) CpG islands and the regulation of transcription. Genes Dev 25: 1010-1022

Eisenberg E, Levanon EY (2013) Human housekeeping genes, revisited. Trends Genet 29: 569_ 574

Erb I, van Nimwegen E (2011) Transcription factor binding site positioning in yeast: proximal promoter motifs characterize TATA-less promoters. PloS One 6: e24279

Erkina TY, Zou Y, Freeling S, Vorobyev VI, Erkine AM (2010) Functional interplay between chromatin remodeling complexes RSC, SWI/SNF and ISWI in regulation of yeast heat shock genes. Nucleic Acids Res 38: 1441-1449

Gross DS, English KE, Collins KW, Lee SW (1990) Genomic footprinting of the yeast HSP82 promoter reveals marked distortion of the DNA helix and constitutive occupancy of heat shock and TATA elements. J Mol Biol 216: 611-631

Grünberg S, Hahn S (2013) Structural insights into transcription initiation by RNA polymerase II. Trends Biochem Sci 38: 603-611

Hahn J-S, Hu Z, Thiele DJ, Iyer VR (2004) Genome-wide analysis of the biology of stress responses through heat shock transcription factor. Mol Cell Biol 24: 5249-5256

Haruki H, Nishikawa J, Laemmli UK (2008) The anchor-away technique: rapid, conditional establishment of yeast mutant phenotypes. Mol Cell 31: 925-932

Henikoff JG, Belsky JA, Krassovsky K, MacAlpine DM, Henikoff S (2011) Epigenome characterization at single base-pair resolution. Proc Natl Acad Sci 108: 18318-18323

Hornung G, Bar-Ziv R, Rosin D, Tokuriki N, Tawfik DS, Oren M, Barkai N (2012) Noise-mean relationship in mutated promoters. Genome Res 22: 2409-2417

Huisinga KL, Pugh BF (2004) A genome-wide housekeeping role for TFIID and a highly 
regulated stress-related role for SAGA in Saccharomyces cerevisiae. Mol Cell 13: 573-585

Imazu H, Sakurai H (2005) Saccharomyces cerevisiae Heat Shock Transcription Factor Regulates Cell Wall Remodeling in Response to Heat Shock. Eukaryot Cell 4: 1050-1056

Ji H, Jiang H, Ma W, Johnson DS, Myers RM, Wong WH (2008) An integrated software system for analyzing ChIP-chip and ChIP-seq data. Nat Biotechnol 26: 1293-1300

Jiang C, Pugh BF (2009) A compiled and systematic reference map of nucleosome positions across the Saccharomyces cerevisiae genome. Genome Biol 10: R109

Juven-Gershon T, Kadonaga JT (2010) Regulation of gene expression via the core promoter and the basal transcriptional machinery. Dev Biol 339: 225-229

Kasinathan S, Orsi GA, Zentner GE, Ahmad K, Henikoff S (2014) High-resolution mapping of transcription factor binding sites on native chromatin. Nat Methods 11: 203-209

Kemmeren P, Sameith K, van de Pasch LAL, Benschop JJ, Lenstra TL, Margaritis T, O’Duibhir E, Apweiler E, van Wageningen S, Ko CW, van Heesch S, Kashani MM, AmpatziadisMichailidis G, Brok MO, Brabers NACH, Miles AJ, Bouwmeester D, van Hooff SR, van Bakel H, Sluiters E, et al (2014) Large-Scale Genetic Perturbations Reveal Regulatory Networks and an Abundance of Gene-Specific Repressors. Cell 157: 740-752

Kent NA, Adams S, Moorhouse A, Paszkiewicz K (2011) Chromatin particle spectrum analysis: a method for comparative chromatin structure analysis using paired-end mode next-generation DNA sequencing. Nucleic Acids Res 39: e26-e26

Kubik S, Bruzzone MJ, Jacquet P, Falcone J-L, Rougemont J, Shore D (2015) Nucleosome Stability Distinguishes Two Different Promoter Types at All Protein-Coding Genes in Yeast. Mol Cell 60: 422-434

Lam FH, Steger DJ, O'Shea EK (2008) Chromatin decouples promoter threshold from dynamic range. Nature 453: 246-250

Langmead B, Salzberg SL (2012) Fast gapped-read alignment with Bowtie 2. Nat Methods 9: 357-359

Lee TI, Causton HC, Holstege FC, Shen WC, Hannett N, Jennings EG, Winston F, Green MR, Young RA (2000) Redundant roles for the TFIID and SAGA complexes in global transcription. Nature 405: 701-704

Lehner B (2010) Conflict between noise and plasticity in yeast. PLoS Genet 6: e1001185

Li H, Durbin R (2009) Fast and accurate short read alignment with Burrows-Wheeler transform. Bioinforma Oxf Engl 25: 1754-1760

MacIsaac KD, Wang T, Gordon DB, Gifford DK, Stormo GD, Fraenkel E (2006) An improved map of conserved regulatory sites for Saccharomyces cerevisiae. BMC Bioinformatics 7: 113

Mieczkowski J, Cook A, Bowman SK, Mueller B, Alver BH, Kundu S, Deaton AM, Urban JA, Larschan E, Park PJ, Kingston RE, Tolstorukov MY (2016) MNase titration reveals differences between nucleosome occupancy and chromatin accessibility. Nat Commun 7: 11485

Mokry M, Hatzis P, de Bruijn E, Koster J, Versteeg R, Schuijers J, van de Wetering M, Guryev V, Clevers H, Cuppen E (2010) Efficient double fragmentation ChIP-seq provides nucleotide resolution protein-DNA binding profiles. PloS One 5: e15092

Morano KA, Grant CM, Moye-Rowley WS (2012) The response to heat shock and oxidative stress in Saccharomyces cerevisiae. Genetics 190: 1157-1195

Müller F, Tora L (2014) Chromatin and DNA sequences in defining promoters for transcription initiation. Biochim Biophys Acta BBA - Gene Regul Mech 1839: 118-128

Murphy KF, Adams RM, Wang X, Balázsi G, Collins JJ (2010) Tuning and controlling gene expression noise in synthetic gene networks. Nucleic Acids Res 38: 2712-2726

Nagalakshmi U, Wang Z, Waern K, Shou C, Raha D, Gerstein M, Snyder M (2008) The transcriptional landscape of the yeast genome defined by RNA sequencing. Science 320: 1344-1349

Nocetti N, Whitehouse I (2016) Nucleosome repositioning underlies dynamic gene expression. 
Genes Dev 30: 660-672

O'Duibhir E, Lijnzaad P, Benschop JJ, Lenstra TL, van Leenen D, Groot Koerkamp MJ, Margaritis T, Brok MO, Kemmeren P, Holstege FC (2014) Cell cycle population effects in perturbation studies. Mol Syst Biol 10: 732

Pereira LA, Klejman MP, Timmers HTM (2003) Roles for BTAF1 and Mot1p in dynamics of TATA-binding protein and regulation of RNA polymerase II transcription. Gene 315: 1-13

Ptashne M, Gann A (1997) Transcriptional activation by recruitment. Nature 386: 569-577

Quinlan AR, Hall IM (2010) BEDTools: a flexible suite of utilities for comparing genomic features. Bioinforma Oxf Engl 26: 841-842

Rando OJ (2010) Genome-wide mapping of nucleosomes in yeast. Methods Enzymol 470: 105118

Raser JM, O'Shea EK (2004) Control of stochasticity in eukaryotic gene expression. Science 304: 1811-1814

Ravarani CNJ, Chalancon G, Breker M, de Groot NS, Babu MM (2016) Affinity and competition for TBP are molecular determinants of gene expression noise. Nat Commun 7: 10417

Raveh-Sadka T, Levo M, Segal E (2009) Incorporating nucleosomes into thermodynamic models of transcription regulation. Genome Res 19: 1480-1496

Reja R, Vinayachandran V, Ghosh S, Pugh BF (2015) Molecular mechanisms of ribosomal protein gene coregulation. Genes Dev 29: 1942-1954

Rhee HS, Pugh BF (2011) Comprehensive genome-wide protein-DNA interactions detected at single-nucleotide resolution. Cell 147: 1408-1419

Rhee HS, Pugh BF (2012) Genome-wide structure and organization of eukaryotic pre-initiation complexes. Nature 483: 295-301

Roeder RG (1996) The role of general initiation factors in transcription by RNA polymerase II. Trends Biochem Sci 21: 327-335

Sainsbury S, Bernecky C, Cramer P (2015) Structural basis of transcription initiation by RNA polymerase II. Nat Rev Mol Cell Biol 16: 129-143

Shivaswamy S, Iyer VR (2008) Stress-Dependent Dynamics of Global Chromatin Remodeling in Yeast: Dual Role for SWI/SNF in the Heat Shock Stress Response. Mol Cell Biol 28: 22212234

Smith BJ, Yaffe MP (1991) A mutation in the yeast heat-shock factor gene causes temperaturesensitive defects in both mitochondrial protein import and the cell cycle. Mol Cell Biol 11: 2647-2655

Smyth GK, Michaud J, Scott HS (2005) Use of within-array replicate spots for assessing differential expression in microarray experiments. Bioinforma Oxf Engl 21: 2067-2075

Solís EJ, Pandey JP, Zheng X, Jin DX, Gupta PB, Airoldi EM, Pincus D, Denic V (2016) Defining the Essential Function of Yeast Hsf1 Reveals a Compact Transcriptional Program for Maintaining Eukaryotic Proteostasis. Mol Cell 63: 60-71

Spedale G, Meddens CA, Koster MJE, Ko CW, van Hooff SR, Holstege FCP, Timmers HTM, Pijnappel WWMP (2012) Tight cooperation between Mot1p and NC2 $\beta$ in regulating genome-wide transcription, repression of transcription following heat shock induction and genetic interaction with SAGA. Nucleic Acids Res 40: 996-1008

Struhl K (1986) Constitutive and inducible Saccharomyces cerevisiae promoters: evidence for two distinct molecular mechanisms. Mol Cell Biol 6: 3847-3853

Struhl K (1995) Yeast transcriptional regulatory mechanisms. Annu Rev Genet 29: 651-674

Sun M, Schwalb B, Schulz D, Pirkl N, Etzold S, Larivière L, Maier KC, Seizl M, Tresch A, Cramer P (2012) Comparative dynamic transcriptome analysis (cDTA) reveals mutual feedback between mRNA synthesis and degradation. Genome Res 22: 1350-1359

Tirosh I, Barkai N (2008) Two strategies for gene regulation by promoter nucleosomes. Genome Res 18: 1084-1091

Tirosh I, Berman J, Barkai N (2007) The pattern and evolution of yeast promoter bendability. 
Trends Genet TIG 23: 318-321

Tirosh I, Weinberger A, Carmi M, Barkai N (2006) A genetic signature of interspecies variations in gene expression. Nat Genet 38: 830-834

Tora L, Timmers HTM (2010) The TATA box regulates TATA-binding protein (TBP) dynamics in vivo. Trends Biochem Sci 35: 309-314

Turatsinze J-V, Thomas-Chollier M, Defrance M, van Helden J (2008) Using RSAT to scan genome sequences for transcription factor binding sites and cis-regulatory modules. Nat Protoc 3: 1578-1588

Venters BJ, Wachi S, Mavrich TN, Andersen BE, Jena P, Sinnamon AJ, Jain P, Rolleri NS, Jiang C, Hemeryck-Walsh C, Pugh BF (2011) A comprehensive genomic binding map of gene and chromatin regulatory proteins in Saccharomyces. Mol Cell 41: 480-492

Viswanathan R, Auble DT (2011) One small step for Mot1; one giant leap for other Swi2/Snf2 enzymes? Biochim Biophys Acta 1809: 488-496

Viswanathan R, True JD, Auble DT (2016) Molecular Mechanism of Mot1, a TATA-binding Protein (TBP)-DNA Dissociating Enzyme. J Biol Chem 291: 15714-15726

Weiner A, Hughes A, Yassour M, Rando OJ, Friedman N (2010) High-resolution nucleosome mapping reveals transcription-dependent promoter packaging. Genome Res 20: 90-100

van Werven FJ, van Teeffelen HAAM, Holstege FCP, Timmers HTM (2009) Distinct promoter dynamics of the basal transcription factor TBP across the yeast genome. Nat Struct Mol Biol 16: 1043-1048

Wiederrecht G, Seto D, Parker CS (1988) Isolation of the gene encoding the S. cerevisiae heat shock transcription factor. Cell 54: 841-853

Wollmann P, Cui S, Viswanathan R, Berninghausen O, Wells MN, Moldt M, Witte G, Butryn A, Wendler P, Beckmann R, Auble DT, Hopfner K-P (2011) Structure and mechanism of the Swi2/Snf2 remodeller Mot1 in complex with its substrate TBP. Nature 475: 403-407

Xi Y, Yao J, Chen R, Li W, He X (2011) Nucleosome fragility reveals novel functional states of chromatin and poises genes for activation. Genome Res 21: 718-724

Zarzov P, Boucherie H, Mann C (1997) A yeast heat shock transcription factor (Hsf1) mutant is defective in both Hsc82/Hsp82 synthesis and spindle pole body duplication. J Cell Sci 110 ( Pt 16): 1879-1891

Zentner GE, Henikoff S (2013) Mot1 redistributes TBP from TATA-containing to TATA-less promoters. Mol Cell Biol 33: 4996-5004 


\section{Supplemental Figures and Tables}

A

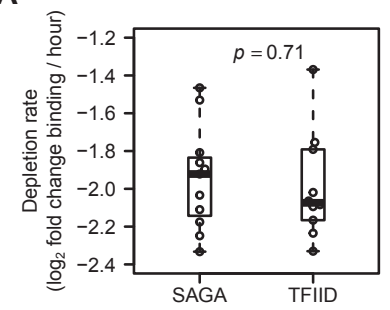

D

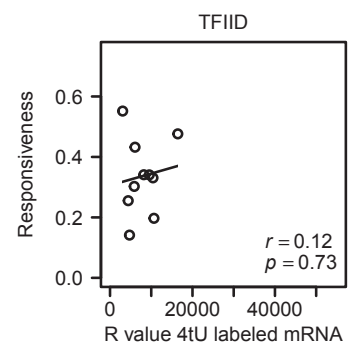

B

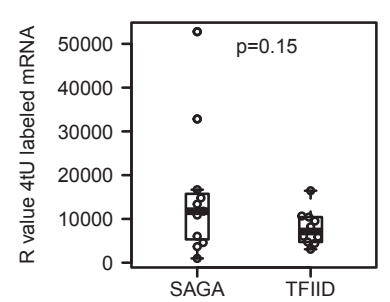

$E$

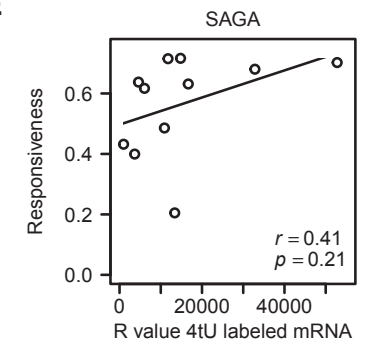

C

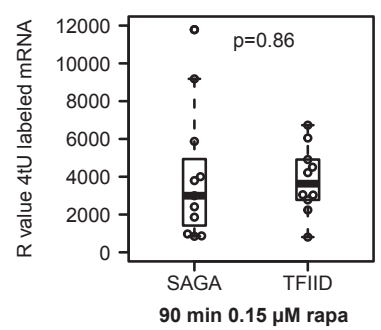

Supplemental Figure S1. Depletion rate and mRNA synthesis levels do not explain differences in responsiveness

(A) Boxplot showing the comparison of the depletion rate of Hsf1 from the promoter of the SAGA and TFIID dominated genes. Depletion rate was calculated by plotting Hsf1 binding versus time and taking the slope of the line fitted through these points. (B) Boxplot of initial mRNA synthesis before addition of rapamycin, as measured by the raw microarray intensity values ( $R$ values), compared between the SAGA and TFIID dominated genes. (C) Boxplot with the mRNA synthesis rates (R values) after 90 minutes of slow depletion. (D and E) Correlation of the mRNA synthesis before depletion (R values) with the responsiveness of (D) the TFIID dominated and (E) the SAGA dominated targets. $p$-values of (A-C) were calculated using a two-tailed t-test, $p$-values of (D-E) were obtained using the function "cor.test" in the statistical language $\mathrm{R}$. 

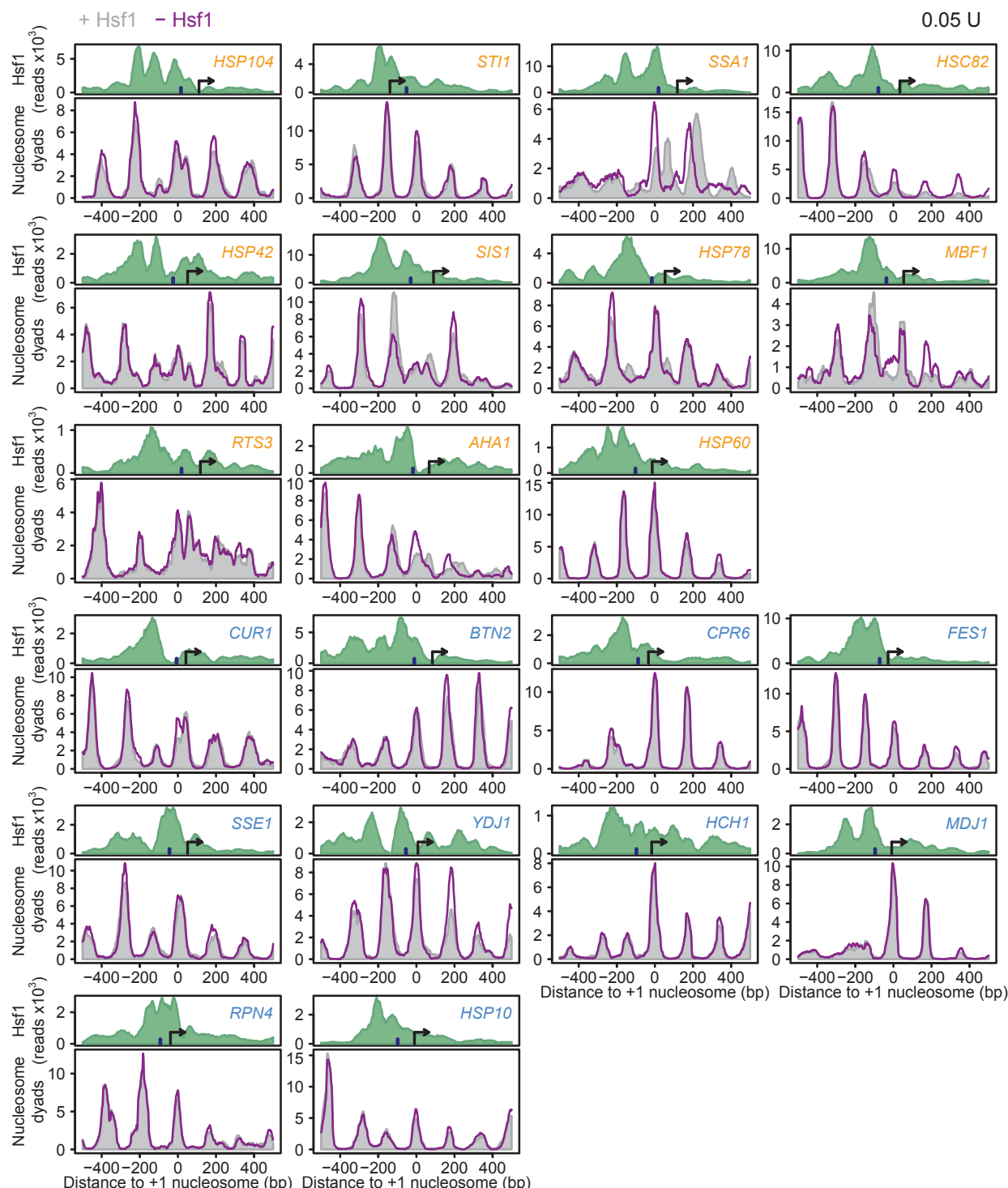

\section{Supplemental Figure S2. Hsf1 removal results in nucleosomal repositioning on SAGA dominated promoters}

MNase-seq nucleosomal DNA midpoint mapping with and without Hsf1 for all 11 SAGA dominated targets (orange) and all 10 TFIID dominated targets (blue). The arrow denotes the transcriptional start site (Nagalakshmi et al., 2008). The dark blue bar indicates the location of the TATA(-like) element (Rhee and Pugh, 2012). For STI1 either the TATA-box or the start site is likely incorrectly called. All the tracks are aligned to the +1 nucleosome. These tracks were used to create the average plot shown in Fig 6 . 
Supplemental Table S1 Robustness of correlation between Hsf1 binding and expression change to outliers.

The correlation $(r)$ and significance of correlation $(p)$ between Hsf1 binding (in reads per peak) and the $\log _{2}$ fold change in expression upon Hsf1 depletion were calculated for the SAGA dominated (upper) and TFIID dominated (lower) genes. Subsequently each of the targets was left out and the correlation was recalculated.

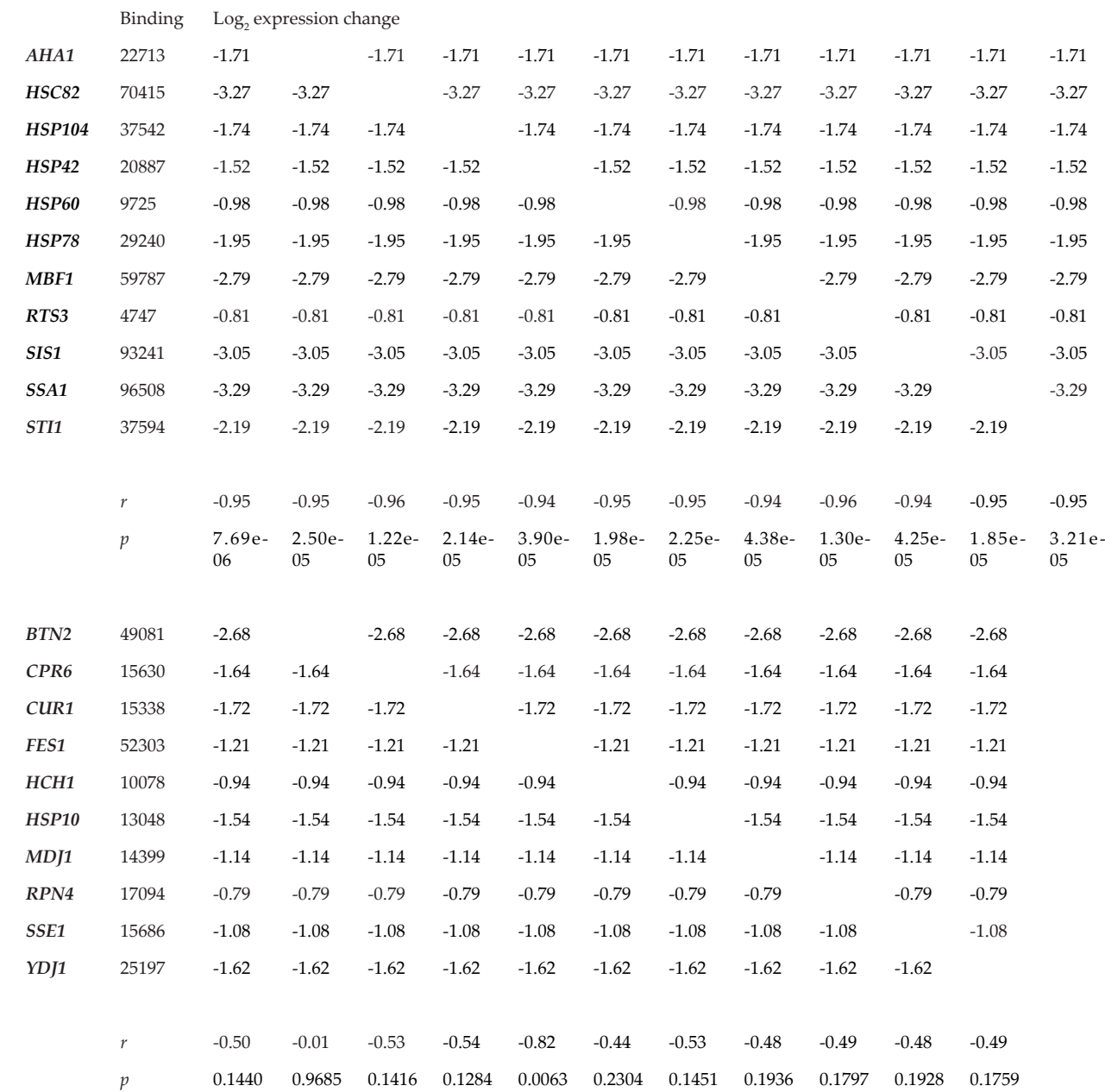


Supplemental Table S2 Primers used for qPCR

\begin{tabular}{|l|l|}
\hline Primer & Sequence 5' - $^{\prime}{ }^{\prime}$ \\
\hline BTN2-F & TTCTTTCGCACCGTCTGTGGTTGT \\
\hline BTN2-R & TTAATTCTTCCATCCTGGGCGGCT \\
\hline HMRF-RT & ACGATCCCCGTCCAAGTTATG \\
\hline HMRR-RT & CTTCAAAAGGAGTCTTAATTTCCCTG \\
\hline Pol1F3 & TTCAGAGGTGGATCAGGGTGT \\
\hline Pol1R2 & GCTTATGGGGATCAGTTTCAGTTTT \\
\hline SSA1-F & AAGAAATAAAGCACGTGTGCGCGG \\
\hline SSA1-R & ACAGCTTCTAAACGTTCCGTGTGC \\
\hline
\end{tabular}





\section{Chapter 3}

\section{Extensive optimization of chromatin immunoprecipitation for quantitatively comparable and robust results}

Wim J. de Jonge, Mariël Brok, Patrick Kemmeren and Frank C.P. Holstege

Princess Máxima Center for Pediatric Oncology, Heidelberglaan 25, 3584 CS

Utrecht, the Netherlands

bioRxiv (2019)

doi: 10.1101/835926 


\section{Abstract}

Chromatin immunoprecipitation (ChIP) is a commonly used technique to investigate which parts of a genome are bound by a particular protein. The result of ChIP is often interpreted in a binary manner: bound or not bound. Due to this focus, ChIP protocols frequently lack the ability to quantitatively compare samples with each other, for example in a time series or under different growth conditions. Here, using the yeast $S$. cerevisiae transcription factors Cbf1, Abf1, Reb1, Mcm1 and Sum1, we optimized the five major steps of a commonly used ChIP protocol: cross-linking, quenching, cell lysis, fragmentation and immunoprecipitation. Quenching with glycine is inefficient and can lead to large degrees of variability, an issue that is resolved by using tris(hydroxymethyl)aminomethane (Tris). Another source of variability is degradation of the protein of interest during the procedure. Enzymatic cell lysis with zymolyase can lead to extensive protein degradation, which is greatly reduced by mechanical lysis through bead beating. Degradation also occurs during sonication of chromatin, affecting large proteins in particular. An optimal mix of protease inhibitors and cross-linking with a higher percentage of formaldehyde reduces the extent of this degradation. Finally we also show that the immunoprecipitation step itself can be greatly improved with magnetic beads and optimized incubation/washing steps. The study results in a highly optimized protocol, which is shorter, easier to perform and has a stronger, more reproducible signal with less background. This protocol is presented in detail. In addition, the results highlight the greatest sources of variability in many other protocols, showing which steps are important to focus on for reproducible and quantitatively comparable ChIP experiments. 


\section{Introduction}

DNA is the carrier of genetic information and how it is decoded, replicated and packaged is largely determined by interactions between DNA and proteins. Studying protein-DNA interactions has therefore been a long-standing topic of interest in the field of molecular biology. There are several ways to assess which sites in the genome are bound by a specific protein in vivo (reviewed in Dey et al, 2012) and one of the oldest and most commonly used techniques is chromatin immunoprecipitation (ChIP). With this technique proteins and DNA are cross-linked and the protein of interest is separated from the chromatin extract using antibodies. The DNA that is bound to the protein of interest is subsequently identified and/or quantified (Fig 1).

\section{The chromatin immunoprecipitation procedure}

ChIP was developed in 1984 to investigate in vivo binding of RNA polymerase to two genes in bacteria (Gilmour \& Lis, 1984). After its first application, the protocol was adapted for other species (reviewed in Kuo \& Allis, 1999) and modified extensively (O'Neill \& Turner, 2003; Rhee \& Pugh, 2011; Kasinathan et al, 2014; Skene \& Henikoff, 2015; He et al, 2015; Gutin et al, 2018). Despite the many varieties of ChIP protocols, the key steps described in detail here, remain largely the same.

After obtaining the cells or tissue of interest (Fig 1, step I), the next step is often fixation of the protein-DNA interactions by cross-linking of the sample (Fig 1, step II), although in some protocols this step is omitted (Hebbes et al, 1988; O'Neill \& Turner, 2003; Kasinathan et al, 2014). There are several ways to cross-link the samples, for example by irradiating with UV or by addition of cross-linking agents such as formaldehyde (Jackson, 1978; Gilmour \& Lis, 1984; Solomon et al, 1988). Although in the first ChIP protocol UV cross-linking was used, the cross-linker of choice is usually formaldehyde. Formaldehyde is preferred due to ease of use and the fact that cross-links can easily be reversed by heating the sample. Additionally, formaldehyde cross-linking can be stopped by addition of a quenching agent (Fig 1, step III), although early adaptations of the protocol did not include a quenching step (Solomon et al, 1988; Dedon et al, 1991).

To gain access to the chromatin, the cross-linked cells have to be lysed (Fig 1, step IV). For most animal cells this is relatively straightforward, but it becomes challenging when working with organisms that have a cell wall such as bacteria, fungi or plants. The cell wall can be disrupted either by using enzymes such as lyticase or zymolyase, or mechanically by for example grinding the cells in liquid nitrogen or by vigorous shaking with glass beads.

With the cells lysed, the chromatin must be fragmented (Fig 1, step V). This step is needed to solubilize the chromatin and to make it accessible for the antibodies. Chromatin can be fragmented either mechanically or enzymatically. Mechanical fragmentation is often achieved using sonication. During sonication, high intensity sound waves exert a force that induces DNA breaks. Alternatively, enzymatic fragmentation with DNAses such as micrococcal nuclease (MNase) can be used to 


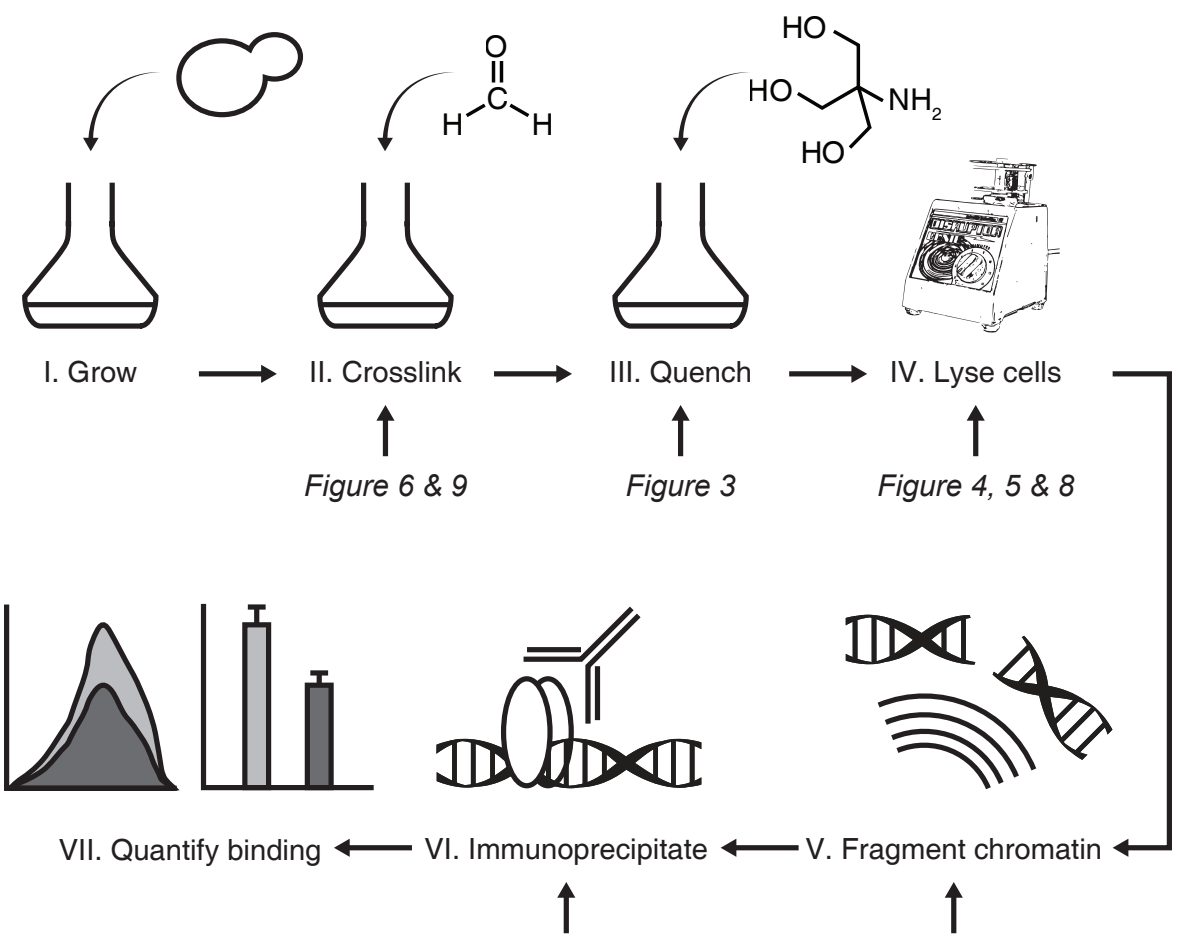

Figure $2 \& 7$

Figure 8

Figure 1. Overview of the ChIP protocol and steps optimized here

Schematic overview of the ChIP protocol. I. First, cells are grown and II. cross-linked using formaldehyde. III. Subsequently the formaldehyde is quenched with a quenching agent and IV. the cells are lysed either mechanically or enzymatically. V. After the cells are lysed, the chromatin is fragmented using sonication and VI. the protein of interest is immunoprecipitated. VII. The DNA that was bound by the protein of interest is isolated and quantified using for example quantitative PCR or sequencing. The arrows show which optimizations are described in which figure.

digest the DNA, by cleaving the DNA that is not protected by proteins.

Having access to the solubilized chromatin, the protein of interest can be immunoprecipitated (Fig 1, step VI). During the immunoprecipitation (IP) step the protein of interest is isolated from the rest of the chromatin using an antibody that specifically binds the protein of interest. The antibody can subsequently be conjugated to beads that can be harvested using either centrifugation or, in the case of magnetic beads, strong magnets. It is important that high-quality antibodies, specific to the protein of interest, are available. Otherwise, it is also possible to tag the protein of interest with a universal epitope-tag such as an HA-, FLAG- or V5-tag (Field et al, 1988; Hopp et al, 1988; Southern et al, 1991). High-quality antibodies are available for these tags, which makes the IP more efficient. Using the same tag for different proteins makes the IP more comparable between these proteins, because the same antibody can be used. However, this requires genetic modification of the cells before treatment to introduce the tag, which might not always be possible. 
Moreover, addition of the tag may alter the activity of the protein, potentially interfering with the result of ChIP.

The last step of any ChIP protocol is to quantify the binding of the protein of interest to DNA (Fig 1, step VII). This is performed by isolating the DNA that was bound to the protein of interest and quantifying this using (q)PCR or sequencing. When using qPCR to quantify the binding, the recovery of DNA bound by the protein of interest is often expressed as the percent of input. This is the signal of the immunoprecipitated sample divided by the signal found in the same sample that was not immunoprecipitated (input). The percentage of input recovered is determined by the efficiency of cross-linking, the efficiency of the IP step and the percentage of cells where the protein of interest was bound to the particular site. Theoretically, if a protein is bound to a certain locus in all cells and the efficiency of cross-linking and IP is $100 \%$, the percent of input recovered is also $100 \%$. High levels of recovery (e.g. $20-50 \%$ ) are only achieved using highly abundant and stable bound proteins such as histones. In general signals are typically much lower (e.g. 0.1-5\%).

\section{Optimization of the chromatin immunoprecipitation procedure}

Over the years many labs have optimized the ChIP protocol, leading to many versions of the method (O'Neill et al, 2006; Acevedo et al, 2007; Dahl \& Collas, 2007; Goren et al, 2010; Adli et al, 2010; Brind'Amour et al, 2015). Most of these efforts focused on optimizing the protocol for a low number of mammalian cells using histone post-translational modifications or RNA polymerase II, which are easier to detect than transiently binding proteins. Often protocols allow the determination of where a protein of interest binds in the genome, but do not enable a quantitative comparison of binding to the same site in different samples. This limitation is not an issue if there is no interest in having accurate comparisons of binding levels, for example, when the main interest is to find where a specific protein is bound in the genome rather than at what level. On the other hand, if the aim is to determine how binding levels change under different conditions, accuracy of the measurements becomes crucial, as well as the ability to quantitatively compare between conditions. For these comparisons a quantitative and reproducible ChIP protocol is needed, but only a few efforts have focused on optimizing the protocol for this purpose.

Here, we describe how to make the ChIP protocol more quantitative and reproducible. This was achieved by optimizing the ChIP protocol using five different gene-specific transcription factors of the yeast Saccharomyces cerevisiae. Centromere binding factor 1 (Cbf1) is required for chromosome segregation (Cai \& Davis, 1990) and can act both as a transcriptional activator and repressor (Kemmeren et al, 2014). ARS binding factor 1 (Abf1) is involved in transcription regulation (Buchman \& Kornberg, 1990; Miyake et al, 2004), DNA repair (Reed et al, 1999) and replication (Rhode et al, 1992). RNA polymerase I enhancer binding protein (Reb1) is involved in transcription regulation of both RNA polymerase I and II transcripts (Chasman et al, 1990). Minichromosome maintenance $1(\mathrm{Mcm} 1)$ is important for recombination and regulation of genes involved in arginine metabolism, cell cycle progression, cell wall maintenance and mating (Kuo \& Grayhack, 1994; Messenguy \& Dubois, 2003). Depending on the mating type Mcm1 can function either as an activator or 
as a repressor (Haber, 2012). Suppressor of mar1-1 (Sum1) is a repressor of middlesporulation genes (Xie et al, 1999), but is also involved in activation of a subset of autonomous replicating sequences (ARS) (Irlbacher et al, 2005). Cbf1, Abf1, Reb1 and $\mathrm{Mcm} 1$ are known as general regulatory factors (GRFs). GRFs are abundant transcription factors (TF) (Ghaemmaghami et al, 2003) that can organize nucleosomes (Kent et al, 2004; Hartley \& Madhani, 2009; Ganapathi et al, 2011) and all but Cbf1 are essential for viability (Passmore et al, 1988; Rhode et al, 1989; Cai \& Davis, 1990; Ju et al, 1990). The yeast GRFs are therefore akin to chromatin pioneer TFs (Zaret \& Mango, 2016).

All experiments described here were carried out using strains that harbor tagged TFs. The TFs were tagged with a V5-tag, which is part of a larger cassette containing GFP and an anchor away tag as well (Haruki et al, 2008). These V5 epitopes are recognized by the antibody used in all experiments described in this work.

Little effort has been done to make quantitative ChIP protocols. Therefore, here we optimized several key steps that are required to make the protocol more quantitative and reproducible. We highlight key points in the protocol that are a source of variation and point out artefacts that may arise during different steps of the procedure. Together, the findings presented here highlight common pitfalls during the ChIP procedure and result in a strongly revised protocol that is suitable for making quantitative and reproducible measurements of protein-DNA binding. This optimized protocol can be found at the end of the materials and methods section.

\section{Results}

\section{Use of magnetic beads improve ChIP enrichment}

One of the key steps in any ChIP protocol is the IP step (Fig 1, step VI). During this step the protein of interest is bound by an antibody, which is then conjugated to beads prior to the precipitation step. The choice of antibody is crucial, as the specificity of the antibody determines the enrichment of genomic regions bound by the protein of interest over non-specific regions (background).

After the antibody binds to its epitope, the antibody is conjugated to beads. Typically these are either agarose or magnetic beads that can be separated using centrifugation or strong magnets, respectively. Agarose beads are often used, but magnetic beads offer the advantage of reduced washing steps and times. As a starting point for protocol optimization, we first tested whether there was a difference in ChIP signal (\% of input) of a Cbf1 anchor away strain (Cbf1-aa) between these two types of beads. Fig 2A shows the difference in ChIP signal when using agarose or magnetic beads. Although the agarose beads have higher signal, the background signal is also increased, and therefore there is no enrichment of the Cbf1 targets (YOS1 and QCR10) over the background (ACT1 and TUB1, p value $>0.70$ ). In contrast, when using magnetic beads there is a clear enrichment of YOS1 over the background ( $p$ value $=0.0007$, indicating that the ChIP enrichment can be improved by switching 
to magnetic beads.

With the switch to magnetic beads, the best condition for eluting DNA from these beads was next investigated. The manufacturer of the magnetic beads suggests using a low $\mathrm{pH}$ glycine buffer to elute the protein of interest from the beads. Alternatively, a TE/SDS buffer at high temperature is often used to reverse the cross-links and elute the DNA from the beads. To determine which of the two is most efficient, these elution methods were compared. Eluting with glycine gives a much lower ChIP signal compared to eluting with a TE/SDS buffer (Fig 2B). This is highlighted by the observation that when glycine eluted beads are subjected to a second elution with TE/SDS, a large portion of the DNA can still be recovered. This shows that a low $\mathrm{pH}$ glycine buffer is not sufficient to elute the DNA from the beads and that it is best to use elution with a TE/SDS buffer.

When using magnetic beads, the recommended incubation time with the chromatin and antibody is 20 minutes. We hypothesized that longer incubation times might increase yield, but since the incubation is performed at room temperature this also increases the risk of proteolytic degradation of the sample. To determine whether a longer incubation time increases the ChIP signal, we tested incubation of the beads with the antibody and chromatin for 20 and 60 minutes. In addition, we also tested whether pre-incubating the antibody with the beads would increase the ChIP signal. The ChIP signal of YOS1 decreases by roughly half when the incubation time is increased from 20 to 60 minutes and pre-incubating the beads with the antibody before binding to the chromatin also decreases the ChIP signal (Fig 2C). This demonstrates that 20 minute incubation of the antibody bound to the chromatin with the beads yields the best signal.

One of the most time-consuming steps of the ChIP procedure is the incubation of antibody with chromatin. Often this step is carried out overnight, to ensure that maximal binding of the protein of interest. If this step can be reduced to only a few hours, the protocol could be shortened by a day. For the anti-V5 antibody, reducing the incubation time increases the ChIP signal (Fig 2D), perhaps by reducing the amount of degradation that takes place during the incubation. When taking the signal and the variation into account, incubation of the chromatin with the V5 antibody for 2 hours is optimal, since this shows the strongest signal (\% of input) with the lowest variation. This is an important improvement because compared to overnight incubation this not only increases the signal but also shortens the protocol by a day.

Besides improving the ChIP signal, another important goal is reducing the variation in both the specific and the background signal between replicate samples. The background signal can be caused by non-target proteins from the cells that stick to the beads aspecifically. This aspecific binding can be prevented by saturating the beads with proteins prior to antibody binding, which potentially reduces the variation in the background levels. Indeed, pre-incubating the beads with bovine serum albumin (BSA) shows a reduction in variation of the background (Fig 2E), indicating that addition of BSA can increase the reproducibility of the protocol by 
A

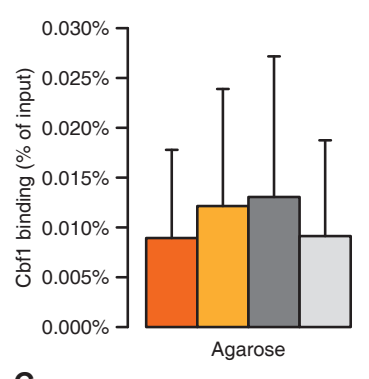

C
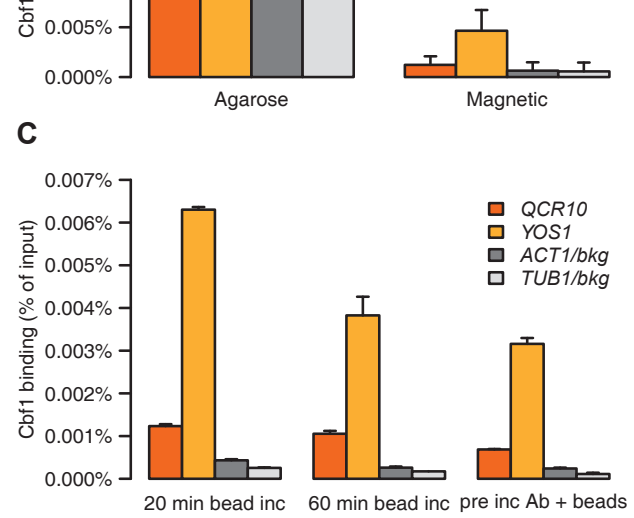

B

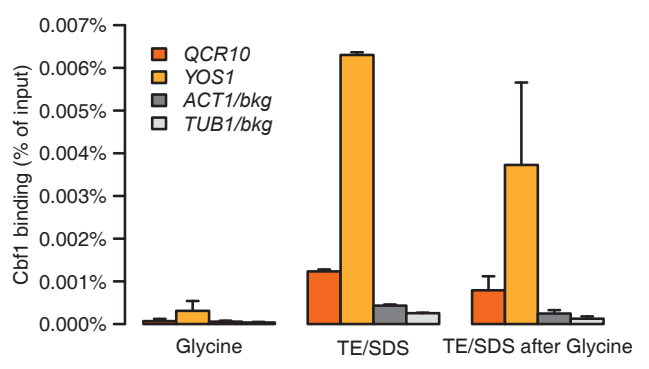

D

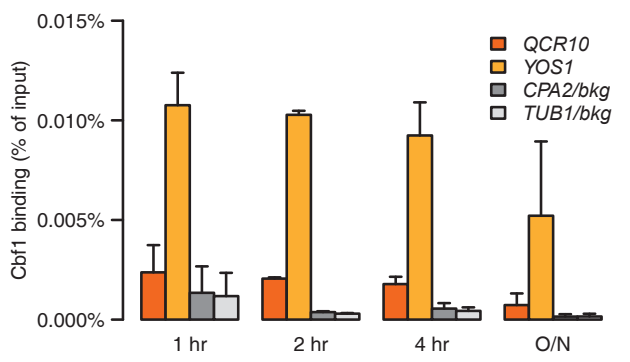

E
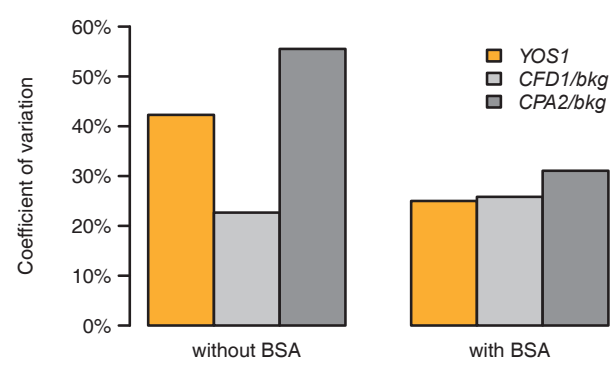

Figure 2. Improved speed and signal using magnetic beads

(A) Percent of input DNA recovered when using agarose versus magnetic beads during the IP. (B) Percent of input DNA recovered when eluting DNA from the beads using glycine, TE/SDS or first glycine and then TE/SDS. (C) Percent of input DNA recovered after incubating the chromatin and antibody with the magnetic beads for 20 minutes, 60 minutes or after first incubating the beads with the antibody and then incubating these with the chromatin. (D) Percent of input DNA recovered after incubating the chromatin with the antibody for 1 hour, 2 hours, 4 hours or overnight $(\mathrm{O} / \mathrm{N})$. (E) Variation in the percent of input as measured by the coefficient of variation after pre-incubating the beads without or with BSA. For all panels a Cbf1-aa strain was used. ACT1, TUB1 and CFD1 were used as background controls (gray) and QCR10 and YOS1 are targets of Cbf1 (McIsaac et al, 2012, orange). The number of replicates used varies per experiment. (A: Agarose and magnetic) have 4 and 8 replicates, respectively. (B and C) were performed using 2 replicates and (D and $E$ ) show the average of 3 replicates. Error bars represent the standard deviation for all experiments with 3 or more replicates (A, D and E) or the distance from the mean for experiments with two replicates (B and C). The same samples are shown in (B: TE/SDS) and (C: $20 \mathrm{~min}$ bead inc).

reducing the variation of the background signal. 
Together these results show that the IP can be improved in several ways. The use of magnetic beads in combination with TE/SDS elution gives a better enrichment compared to agarose beads. The optimal incubation time of the anti-V5 antibody with chromatin is 2 hours and addition of BSA to the beads prior to antibody binding reduces variation. Besides the improved practicality and increased reproducibility, these optimizations also led to a greatly increased ChIP signal over background (compare Fig 2A and 2D).

\section{Glycine is a poor quencher}

An important first step of most ChIP procedures is cross-linking of proteins to DNA (Fig 1, step II). Cross-linking is stopped by quenching the cross-linking agent (Fig 1, step III). Proper quenching is an important step, especially in time course experiments where subsequent time points need to be quantitatively comparable. Most often the samples are cross-linked with formaldehyde and quenched by addition of glycine (Kuo \& Allis, 1999; Acevedo et al, 2007; Rhee \& Pugh, 2011; Poorey et al, 2013; Lara-Astiaso et al, 2014; He et al, 2015; Skene \& Henikoff, 2015; Gutin et al, 2018). Glycine can quench formaldehyde because the amino group of glycine can react with formaldehyde, which prevents it from forming cross-links with other macromolecules. Remarkably, the concentration of glycine used is often sub-stoichiometric to formaldehyde (Kuo \& Allis, 1999; Acevedo et al, 2007; Rhee \& Pugh, 2011; Poorey et al, 2013; He et al, 2015; Skene \& Henikoff, 2015; Gutin et al, 2018) and it has been shown that glycine is not necessarily an efficient quencher at these concentrations (Sutherland et al, 2008; Zaidi et al, 2017).

To investigate the effectiveness of quenching, a time course was performed. Yeast cultures were cross-linked with standard amounts of $1 \%$ formaldehyde $(\sim 333 \mathrm{mM})$ for 1 minute and subsequently quenched with $125 \mathrm{mM}$ glycine for 1, 5 or 10 minutes. If glycine quenches formaldehyde efficiently there should be no difference in ChIP signal between the samples. However, there is a clear increase in ChIP signal with longer glycine incubation times (Fig 3A), which means that the cross-linking is not stopped by addition of glycine at a concentration of $125 \mathrm{mM}$. Potentially formaldehyde can be quenched more efficiently using a higher glycine concentration. When 250 $\mathrm{mM}$ glycine is added for 5 minutes the ChIP signal seems to decrease only slightly compared to quenching with $125 \mathrm{mM}$ for 5 minutes (Fig 3B), and the ChIP signal still increases compared to 1 minute of quenching (compare Fig $\mathbf{3 A}$ and $\mathbf{3 B}$ ). This confirms that formaldehyde can still form cross-links and that glycine, therefore, does not fully quench the formaldehyde when added at these levels.

\section{Tris is a much more efficient quencher}

Since glycine is not quenching properly when added at these levels, the quenching procedure has to be improved, since this will otherwise increase variation (see Discussion). Others have suggested two ways to quench more efficiently, either using very highly concentrated glycine solutions (Zaidi et al, 2017) or using excess Tris concentrations (Sutherland et al, 2008). Although highly concentrated glycine solutions $(2.93 \mathrm{M})$ do quench better than sub-stoichiometric glycine solutions (Zaidi et al, 2017), this requires concentrating the culture before cross-linking and 
A
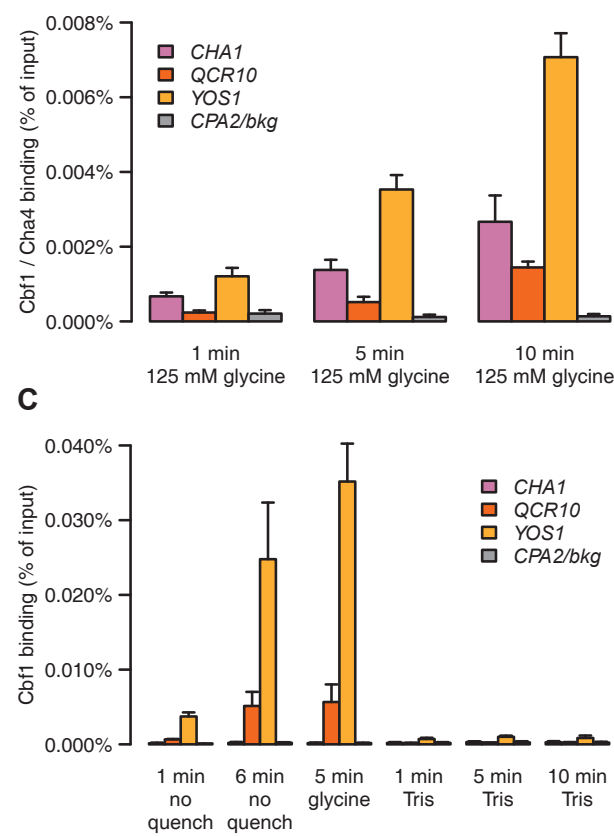

B
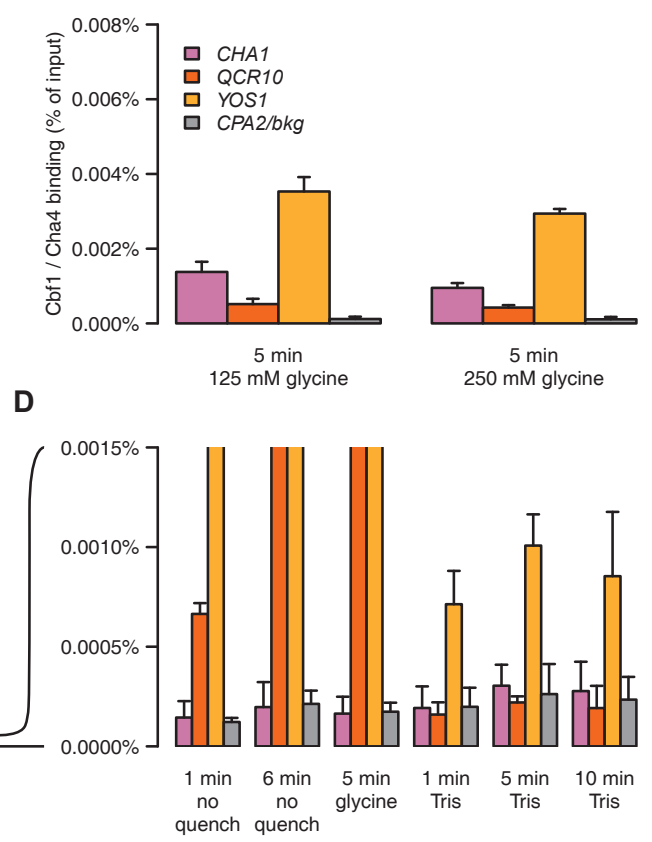

Figure 3. Tris is a superior quencher compared to glycine

(A) Percent of input DNA recovered after quenching for 1, 5 or 10 minutes with $125 \mathrm{mM}$ glycine. (B) Percent of input DNA recovered when quenching with $125 \mathrm{mM}$ or $250 \mathrm{mM}$ glycine for 5 minutes. (C) Percent of input DNA recovered after cross-linking for 1 or 6 minutes without quenching, or 1 minute of cross-linking followed by quenching with glycine for 5 minutes or quenching with Tris for 1,5 or 10 minutes. (D) Zoom in of (C) to $0.0015 \%$ recovery of input DNA. (A and B) were performed with a Cbf1aa Cha4-V5 strain and (C and D) with a Cbf1-aa strain. CPA2 was used as a background control (gray), QCR10 and YOS1 are Cbf1 targets (McIsaac et al, 2012, orange) and CHA1 is a target of Cha4 (MacIsaac et al, 2006, pink). Error bars represent the standard deviation of 3 replicates. The same samples are shown for $5 \mathrm{~min} 125 \mathrm{mM}$ in (A and $\mathrm{B})$.

addition of $440 \mathrm{ml}$ glycine per $10 \mathrm{ml}$ concentrated culture. This makes the procedure impractical and potentially stresses the cells during the steps needed to concentrate the cell culture. Quenching with Tris on the other hand, has been reported to be more efficient than with glycine (Sutherland et al, 2008) and thus can be achieved with lower concentrations. Tris can quench formaldehyde more effectively because a single Tris molecule is able to bind two formaldehyde molecules and it binds formaldehyde more stably than glycine (Hoffman et al, 2015). However, concerns have been raised that quenching with Tris will even de-cross-link because formaldehyde binding by Tris is so efficient (Hoffman et al, 2015; Zaidi et al, 2017). In contrast, others have demonstrated that prolonged incubation of cross-linked samples in Tris solution without heating does not de-cross-link (Sutherland et al, 2008; Kawashima et al, 2014).

To investigate if Tris quenches formaldehyde completely, and whether it de-crosslinks the protein-DNA interactions, we performed the following experiment. Cbf1- 
aa cultures were cross-linked for 1 minute, without quenching, or with quenching either for 5 minutes using $125 \mathrm{mM}$ glycine, or 1, 5 or 10 minutes using $750 \mathrm{mM}$ Tris. Cultures that were cross-linked for 6 minutes without quenching were also taken along as a control for the samples that were quenched for 5 minutes. By comparing the samples that were cross-linked for 6 minutes without quenching with the samples that were quenched with glycine or Tris for 5 minutes, it is possible to determine to what extent glycine or Tris are able to quench formaldehyde. Surprisingly, there is no significant difference in the ChIP signal of YOS1 ( $\mathrm{p}$ value $=0.13$ ) and QCR10 ( $\mathrm{p}$ value $=0.78$ ) between samples that were cross-linked for 6 minutes and not quenched and samples that were cross-linked for 1 minute and quenched for 5 minutes with $125 \mathrm{mM}$ glycine (Fig 3C). This indicates that glycine is not quenching at all this concentration. In contrast, when using Tris the cross-linking is efficiently stopped, as is evident by the lower ChIP signal.

The ChIP signal of the Tris quenched samples are even lower than the samples that were cross-linked for 1 minute without quenching (Fig 3C). Although this could indicate that Tris de-cross-links the proteins from DNA, this is nevertheless unlikely. Firstly, for the non-quenched samples cross-linking is not stopped after 1 minute incubation with formaldehyde. After incubation, the samples are centrifuged for several minutes, and subsequently resuspended in Tris-buffered saline (TBS). The Tris present in this buffer will fully stop the cross-linking reaction. This means that the non-quenched samples were effectively cross-linked for several minutes. Crosslinking in the Tris quenched samples, on the other hand, was effectively stopped after 1 minute. The longer effective cross-linking time of the non-quenched sample likely leads to the observed higher ChIP signal. Secondly, prolonged incubation of the samples with Tris does not lower the ChIP signal (Fig 3D). If addition of Tris would de-cross-link proteins from DNA, the ChIP signal would have decreased with longer incubation times.

Together, these results show that glycine is a very poor quencher, that quenching with Tris is far more efficient and that short quenching times with Tris do not decross-link the samples. Thus, using Tris as a quenching agent improves the accuracy and reproducibility of the ChIP protocol, which is important for quantitative comparisons.

\section{Cbf1 is degraded during zymolyase treatment}

Previous experiments focused on the immunoprecipitation and quenching steps. Another aspect that may be optimized is cell lysis (Fig 1, step IV). In contrast to mammalian cells, yeast cells have a strong cell wall that needs to be disrupted in order to gain access to the chromatin. In the experiments described so far, cells were lysed enzymatically by addition of zymolyase. Zymolyase digests the cell wall, creating spheroplasts that can subsequently be lysed by sonicating them in a lysis buffer. We reasoned that by extending the zymolyase treatment, a bigger proportion of cells would have their cell walls digested, potentially leading to a more efficient recovery of the protein of interest. 
A

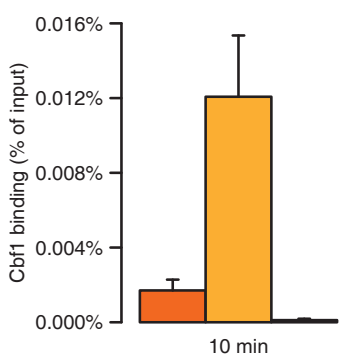

B

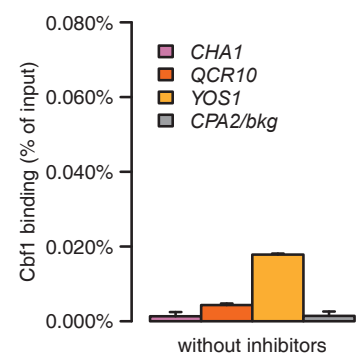

C
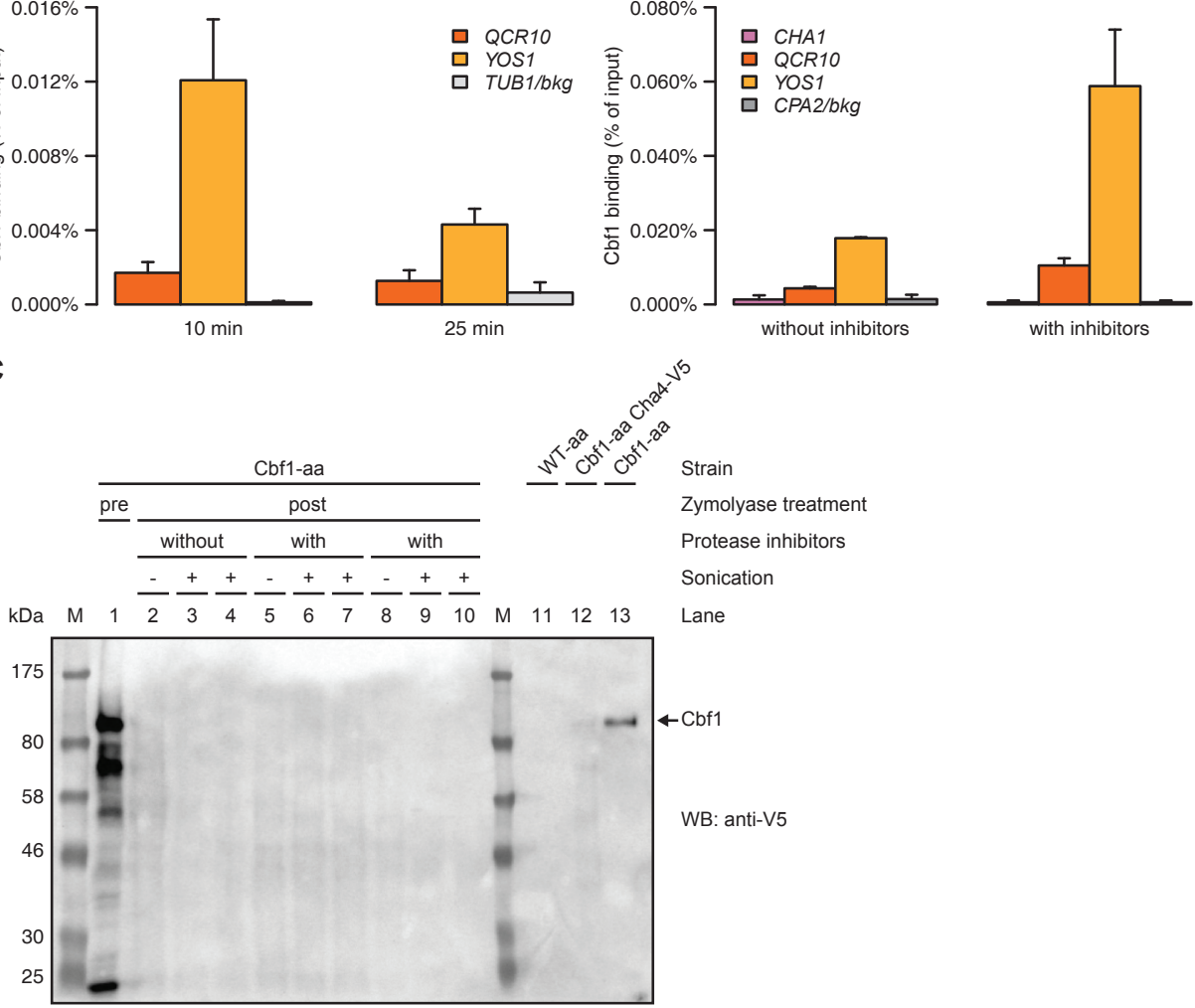

Figure 4. Zymolyase treatment causes degradation of proteins of interest

(A) Percent of input DNA recovered after treating the cells with zymolyase for 10 or 25 minutes. (B) Percent of input DNA recovered after treating the cells with zymolyase in the presence or absence of protease inhibitors. (C) Western blot showing the presence of Cbf1. Tagged Cbf1 has a mass of approximately 83 $\mathrm{kDa}$. The location of tagged Cbf1 on the blot is indicated on the right. Different steps of the ChIP protocol were loaded on the gel: before lysis (pre-zymolyase treatment), after lysis (post-zymolyase, pre-sonication) and after the sonication (post-sonication). Cells were treated with zymolyase in the absence or presence of protease inhibitors. On the right side crude lysates were loaded as negative (WT-aa, the untagged parental strain) and positive (Cbf1-aa) controls. The blot was incubated with an antibody specific for V5. For (A and B) a Cbf1-aa strain was used without Cha4-V5 and for (C) both a Cbf1-aa and WT-aa, as indicated above the blot. In (A and B) CPA2 and TUB1 were used as background controls (gray), QCR10 and YOS1 are Cbf1 targets (McIsaac et al, 2012, orange) and CHA1 is a target of Cha4 (MacIsaac et al, 2006, pink). The number of replicates used varies per experiment. (A) was performed with 3 replicates. (B: without inhibitors) had 2 replicates and (B: with inhibitors) shows the average of 4 replicates. Error bars represent the standard deviation for all experiments with 3 or more replicates (A and B: with inhibitors) or the distance from the mean for experiments with two replicates (B: without inhibitors).

To test this, ChIP signals were compared for Cbf1-aa cells that were treated with zymolyase for either 10 or 25 minutes (Fig 4A). In contrast to our expectations, zymolyase treatment for 25 minutes decreased the ChIP signal compared to 10 minutes treatment. This suggests that there is a component present during zymolyase treatment that decreases the ChIP efficiency. One possibility is proteases, which are known to be present in zymolyase preparations. To test whether proteases 
were degrading the protein of interest during the zymolyase treatment, and thereby reducing the ChIP signal, we lysed cells using zymolyase with and without addition of protease inhibitors. Cells that were treated in the presence of protease inhibitors had a two- to threefold increase in ChIP signal (Fig 4B). This suggests that indeed $\mathrm{Cbf} 1$ is degraded by proteases during zymolyase treatment and that this decreases the ChIP signal.

To directly assess whether Cbf1 is degraded during zymolyase treatment, the amount of intact protein was measured at different steps during the protocol using Western blotting. The amount of intact Cbf1 was determined before zymolyase treatment, after zymolyase treatment with and without protease inhibitors as well as after sonication. Samples before and after sonication were taken along because the sonication process can potentially induce protein degradation (Pchelintsev et al, 2016). Before zymolyase treatment there is a strong Cbf1 band present (Fig 4C, lane 1), but, to our surprise, after zymolyase treatment there is no longer any detectable protein, even in the presence of protease inhibitors (Fig 4C, lanes 2-10). This indicates that most of the Cbf1 is degraded during zymolyase treatment, likely by the proteases present in the zymolyase preparation. Addition of protease inhibitors is likely not sufficient to prevent proteolytic degradation of the proteins of interest. This suggests that lysing the cells with a different mechanism that does not degrade the proteins being studied could strongly improve the ChIP signal.

\section{Mechanical cell disruption improves ChIP signal}

An alternative mechanism to lyse cells is mechanical disruption. A common way to disrupt cells mechanically is by adding small glass beads to the cells in a tube containing lysis buffer and agitating the tubes at high speed, which is called bead beating. Since bead beating does not involve incubating the cells with proteases, we reasoned that this might improve the ChIP signal. Indeed, when comparing the zymolyase protocol with the beat beating protocol using a Cbf1-aa strain that also had a V5 tagged Cha4, there is a more than 10-fold increase in the ChIP signal of both TFs tested (Fig 5A). This shows that the ChIP signal can be improved by replacing zymolyase with mechanical disruption such as bead beating. However, the bead beat protocol is also more cumbersome and time-consuming.

Therefore, our next aim was to determine if the number of steps in the protocol could be reduced, speeding up the procedure and possibly also increasing the reproducibility. A step that potentially can be omitted during cell lysis is the last step, when chromatin is separated from the rest of the cell lysate. During this step the lysate is centrifuged at high speed for 20 minutes, which concentrates the crosslinked chromatin. The supernatant, called the whole cell lysate (WCL), is discarded while the pellet, called the chromatin extract (CE), is resuspended and used for the IP. However, resuspending this pellet is often quite difficult, which could be a potential source of variability. If the IP is similarly efficient without separating the CE from the rest of the WCL, this could allow for a faster and potentially more robust protocol. To assess if the protocol is similarly efficient without the last centrifugation step, the ChIP signal of the CE and the WCL from the full bead beat protocol was compared 
A

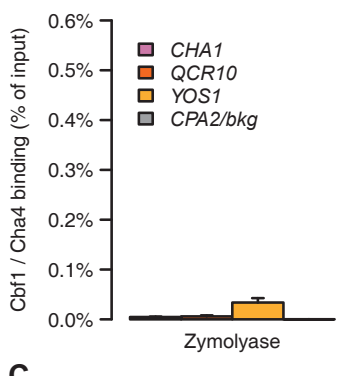

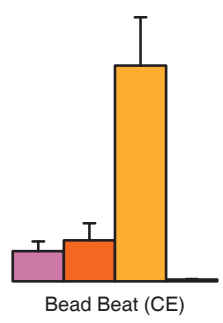

B

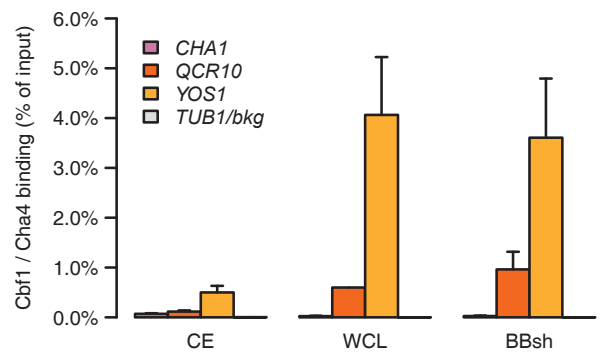

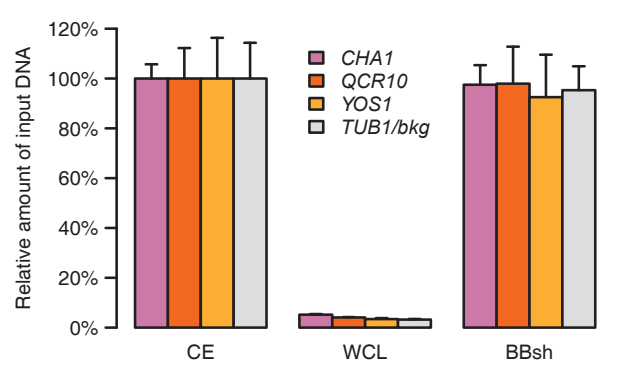

D

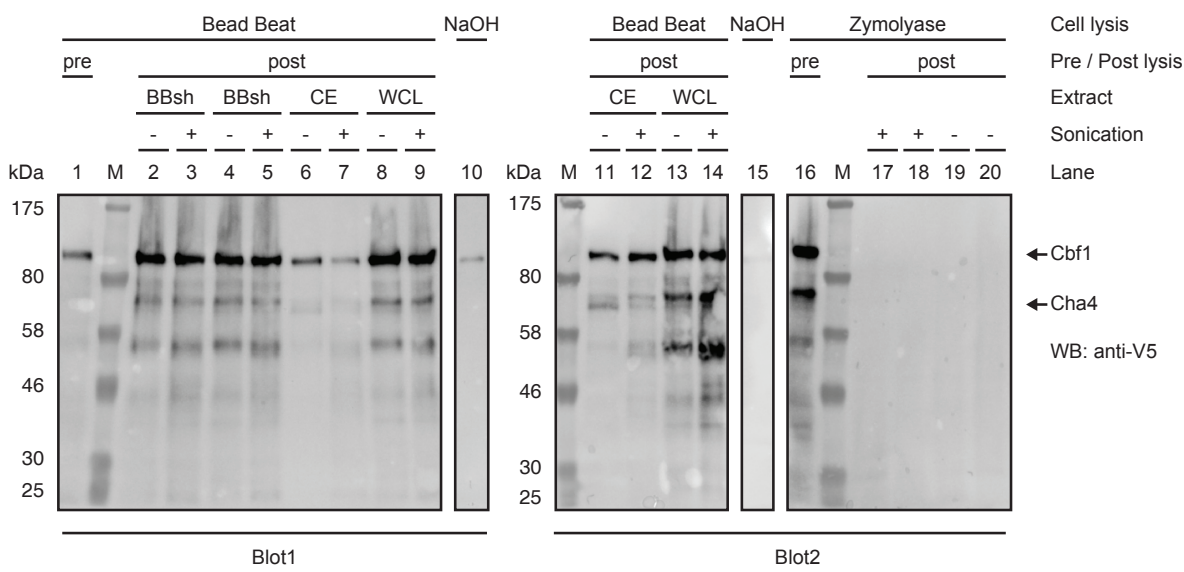

Figure 5. Cell lysis by bead beating greatly improves ChIP signal

(A) Percent of input DNA recovered after lysing the cells using either zymolyase or bead beating. (B) Percent of input DNA recovered after lysing the cells using the bead beat protocol with separation of chromatin extract (CE) and whole cell lysate (WCL), or without (BBsh). (C) Relative recovery of the input DNAs. The amount of input DNA recovered in the CE sample was set to $100 \%$ and the amount of input DNA recovered in the WCL and BBsh was scaled accordingly. (D) Western blot showing the presence of Cbf1 and Cha4. Tagged Cbf1 has a mass of approximately $83 \mathrm{kDa}$ and Cha4-V5 has a mass of approximately $85 \mathrm{kDa}$. The location of these proteins on the blot is indicated on the right. Cha4 is less abundant than Cbf1, which makes it hard to detect on the blots. On both blots a crude lysate (NaOH) of a Cbf1-aa strain, without Cha4-V5 was used as a positive control. The different bead beat extracts and the zymolyase treated lysate before and after sonication are shown in duplicate. The blots were incubated with an antibody specific for V5. For (A, B and C) a Cbf1-aa Cha4-V5 strain was used, for (D) all but the $\mathrm{NaOH}$ samples were from a Cbf1-aa Cha4-V5 strain and for the $\mathrm{NaOH}$ samples a Cbf1-aa strain was used. In (A, B and C) CPA2 and TUB1 were used as background controls (gray), QCR10 and YOS1 are Cbf1 targets (McIsaac et al, 2012, orange) and CHA1 is a target of Cha4 (MacIsaac et al, 2006, pink). The number 
to the signal obtained with a shorter protocol without the last centrifugation step (BBsh). This comparison was made using a Cbf1-aa strain that also had a V5 tagged Cha4. Interestingly, the WCL and BBsh samples showed an even stronger ChIP enrichment of the Cbf1 targets YOS1 and QCR10 compared to the CE (Fig 5B). The Cha4 target $C H A 1$, on the other hand, does not show increased signal using the shorter protocol or the WCL. This indicates that, at least for Cbf1, a higher ChIP signal can be obtained by using the short protocol or the WCL. It is important to note, however, that while the WCL has a high percent of input recovered, it has much lower absolute levels of DNA (Fig 5C), because most of the DNA is contained in the CE. Since more than $95 \%$ of the DNA is lost, the WCL is not suited for doing IPs.

The higher ChIP signal obtained with the bead beat protocols compared to the zymolyase protocol could be explained by an increased stability of the proteins. To verify if this was the case, the protein stability was compared between the different bead beat protocols and versus the zymolyase protocol. There is an improvement in protein stability with the bead beat protocols compared to the zymolyase protocol, since the protein levels are now readily detectable by Western blot (Fig 5D). Nevertheless, there is still some degradation taking place during the bead beat protocols (Fig 5D, lanes 2-9 and 11-14), but only to a limited extent compared to the zymolyase treatment (Fig 5D, lanes 17-20). This increase in stability explains the higher ChIP signals observed with the bead beat protocols and shows that the protocol can be improved by lysing the cells mechanically. Using a shorter version of the protocol allows for a faster protocol that has even stronger ChIP signal for Cbf1, but not for Cha4.

\section{Cbf1 may re-bind DNA during the IP step}

The strong increase in Cbf1 ChIP signal using the short bead beat protocol suggests that this protocol is the best for Cbf1 ChIP. However, in contrast to Cbf1, the ChIP signal of Cha4 does not increase with the short protocol (Fig 5B). This suggests that perhaps the increase in Cbf1 ChIP signal is artefactual, and may not reflect actual in vivo binding. To test this, Cbf1-aa cells were grown with and without cross-linking, and subjected to the short bead beat protocol. Indeed, even without cross-linking Cbf1 binding can easily be detected (Fig 6A). In contrast, two other TFs, Abf1 (Fig 6C) and Reb1 (Fig 6D), do not show any binding without cross-linking. The binding of Cbf1 without cross-linking could be explained either by strong DNA binding, which does not need cross-linking to be retained, or by binding of unbound Cbf1 to these sites during the IP procedure. To test the latter, Cbf1 was depleted from the nucleus for 60 minutes using the anchor away technique (Haruki et al, 2008). The depleted cells were cross-linked for 5 minutes and the ChIP signal was compared to $\mathrm{Cbf1}$ binding without depletion. Cbf1 is depleted to background levels from the

of replicates used varies per experiment. (B and C: WCL) were performed using 2 replicates. (A) has 3 replicates and (B and $\mathrm{C}: \mathrm{CE}$ and $\mathrm{BBsh}$ ) show the average of 4 replicates. Error bars represent the standard deviation for all experiments with 3 or more replicates (A, B and C: CE and BBsh) or the distance from the mean for experiments with two replicates (B and C: WCL). 
A

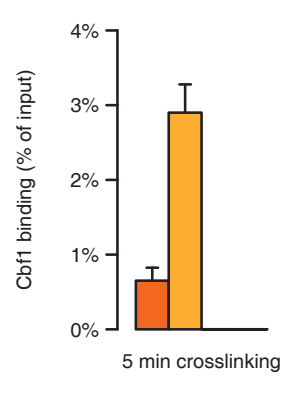

C

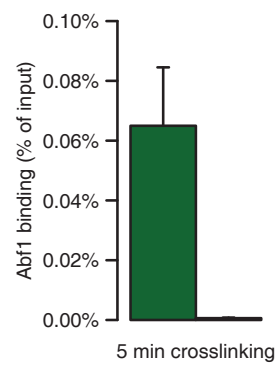

E
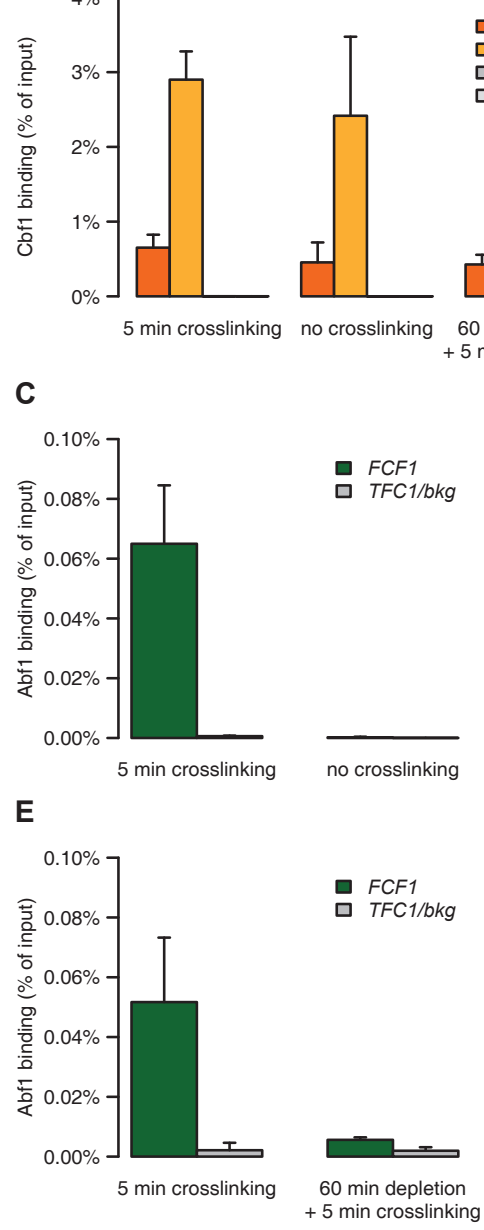

B

$\begin{array}{ll}\square & \text { QCR10 } \\ \square & \text { YOS1 }\end{array}$

$\square T F C 1 / \mathrm{bkg}$

$T U B 1 / \mathrm{bkg}$

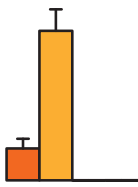

60 min depletion

+5 min crosslinking

D

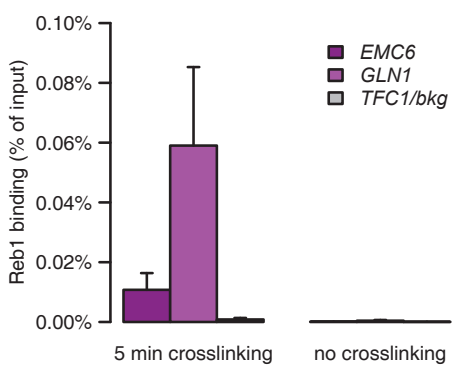

$\mathbf{F}$

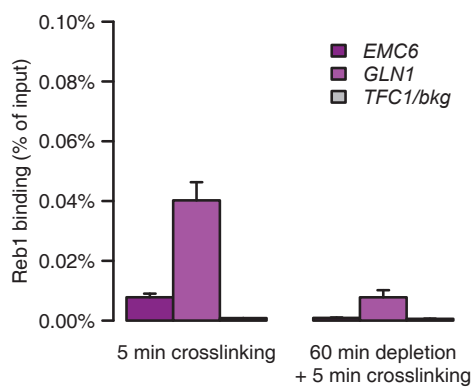

Figure 6. Cbf1 can re-bind DNA during immunoprecipitation

(A) Percent of input DNA recovered after cross-linking the cells for 5 minutes, in the absence of crosslinking or when Cbf1 is depleted from the nucleus for 60 minutes prior to cross-linking for 5 minutes. (B) Fluorescence microscopy images of Cbf1-FRB-GFP-3V5 before, 15 minutes and 60 minutes after induction of nuclear depletion. Scale bar: $10 \mu \mathrm{m}$. (C and D) Percent of input DNA recovered with 5 minutes or without cross-linking for (C) Abf1 and (D) Reb1. (E and F) Percent of input DNA recovered before or after 60 minutes of depletion of (E) Abf1 and (F) Reb1 from the nucleus prior to cross-linking for 5 minutes. For (A and B) a Cbf1-aa strain, for (C and E) an Abf1-aa strain and for (D and F) a Reb1-aa strain was used. In all experiments the cells were lysed using the short bead beat protocol. TFC1 and TUB1 were used as background controls (gray), QCR10 and YOS1 are Cbf1 targets (McIsaac et al, 2012, orange), FCF1 is an Abf1 target (Kasinathan et al, 2014, green) and EMC6 and GLN1 are Reb1 targets (Kasinathan et al, 2014, purple). (A: 5 min cross-linking and 60 min depletion, C, D, E and F) were performed using 3 replicates and (A: no cross-linking) had 6 replicates. Error bars represent the standard deviation.

nucleus after approximately 15 minutes (Fig 6B). Thus, depletion of Cbf1 from the nucleus for 60 minutes should abolish Cbf1 binding to DNA. Therefore, if there is still ChIP signal detected after nuclear depletion, this would indicate that Cbf1 
rebinds DNA during the IP step. Indeed, with Cbf1 depleted from the nucleus, the binding levels are only reduced by one third compared to no depletion (Fig 6A), while Abf1 (Fig 6E) and Reb1 (Fig 6F) show at least a fourfold reduction in binding after nuclear depletion.

These observations suggest that free and depleted, cytosolic Cbf1 can re-bind DNA during the IP step because in the short bead beat protocol the cytosolic and nuclear fraction are mixed during the IP step. Unfortunately, this makes the short bead beat protocol in this form unsuitable for determining in vivo Cbf1 binding levels, since binding may arise in vitro during the IP procedure. However, it can still be used for other proteins, such as Abf1, Cha4 and Reb1, which do not bind DNA during the IP step on the promoters tested.

\section{Re-optimization of the immunoprecipitation step}

So far, several steps of the ChIP protocol have been optimized and changed (Fig 1-6). Because many such changes were made after the initial optimizations of the immunoprecipitation step, we re-evaluated the way the immunoprecipitation is performed (Fig 1, step VI). Since the short bead beat protocol is not well suited for determining binding levels of $\mathrm{Cbf} 1$, the optimizations were carried out using a Sum1-aa strain. Sum1 has a lower abundance than Cbf1 (Ghaemmaghami et al, 2003), and may therefore have a lower ChIP signal. Thus, using Sum1 would allow for optimization of the IP procedure also for less abundant proteins.

As a starting point, the elution was re-investigated. As shown in Fig 2B, elution of the immunoprecipitated DNA with TE/SDS is more efficient than eluting with low $\mathrm{pH}$ glycine. Still, this does not necessarily show that all DNA is efficiently eluted. To confirm that all DNA is eluted, an IP was performed with overnight elution using TE/SDS and after the first elution a second elution was performed on the same beads, again with TE/SDS. If overnight elution is not sufficient to elute all DNA, a significant amount of DNA should be recovered in the secondary elution. Fig 7A shows that when a second elution is performed, only about $2 \%$ of the initial elution is recovered. This demonstrates that overnight elution is sufficient, as the vast majority of DNA is eluted in this step.

After confirming the efficiency of the elution, we checked whether washing of the beads could be further optimized. The manufacturer of the magnetic beads recommends two washes with PBS followed by two washes with PBS-Tween, which has been used as the standard wash in the experiments described so far. To explore which washing conditions are best for the IP of Sum1, this standard wash was compared to a less stringent wash and two kinds of more stringent washes: high wash1 and high wash2 (see Materials and Methods). Doing a less stringent wash leads to a higher ChIP signal, but also to an increase in background signal (Fig 7B). On the other hand, both stringent washes had similar Sum1 ChIP signals compared to the standard wash, but a reduction in the background signal of about $40 \%$. Therefore, at least for Sum1, washing more stringently can improve the signal to noise ratio. 
Lastly, incubation of the chromatin and antibody with the beads was optimized. This incubation is normally carried out at room temperature (RT) for 20 minutes. Proteases are more active at RT, thus reducing the temperature during this step could lead to less degradation and higher ChIP signal. On the other hand, lowering the temperature could also reduce the efficiency of antibody binding to the beads. To test the effect of temperature on this step, the ChIP signal was compared between samples that were incubated at RT for 20 minutes and at $4^{\circ} \mathrm{C}$ for 20 or 60 minutes. When the chromatin and antibody are incubated with the beads at $4^{\circ} \mathrm{C}$ for 20 minutes there is a small decrease in ChIP signal compared to 20 minute incubation at RT (Fig 7C). Incubating at $4^{\circ} \mathrm{C}$ for 60 minutes increases the ChIP signal, but also increases the background (Fig 7D). This indicates that there is no added benefit of doing the incubation at $4^{\circ} \mathrm{C}$.

A
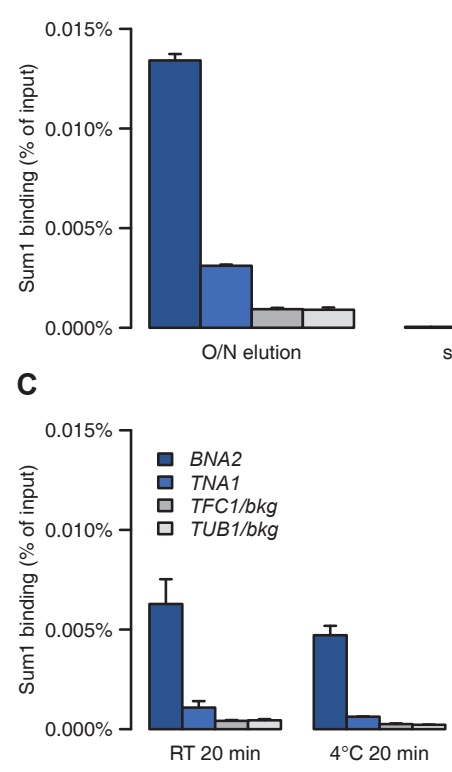

B

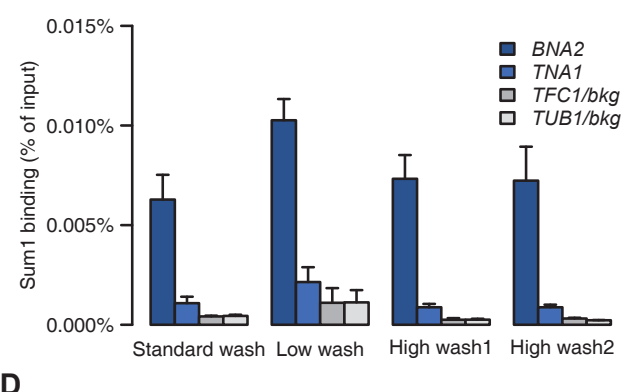

D

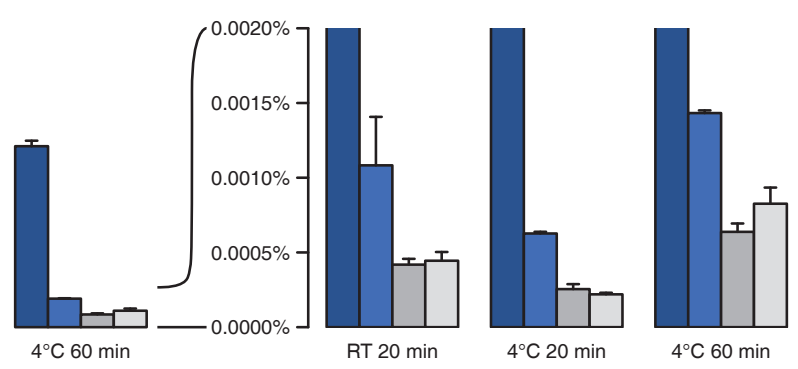

Figure 7. Re-optimization of the immunoprecipitation for the bead beat protocol

(A) Percent of input DNA recovered after eluting the DNA from the beads overnight or after a second elution was performed on the same beads. (B) Percent of input DNA recovered after using different washes during the IP, see Materials and Methods for details. (C) Percent of input DNA recovered after incubating the chromatin and the antibody with the beads at room temperature (RT) for 20 minutes or at $4^{\circ} \mathrm{C}$ for 20 or 60 minutes. (D) Zoom in of (C) to $0.002 \%$ recovery of input DNA. For all panels a Sum1aa strain was used. TFC1 and TUB1 were used as background controls (gray) and BNA2 and TNA1 are Sum1 targets (MacIsaac et al, 2006, blue). The number of replicates used varies per experiment. (A, B: high wash 1 and $\mathrm{C}$ and $\mathrm{D}: 4^{\circ} \mathrm{C} 20 \mathrm{~min}$ and $4^{\circ} \mathrm{C} 60 \mathrm{~min}$ ) were performed using 2 replicates. (B: standard wash and $C$ and D: RT $20 \mathrm{~min}$ ) both have 3 replicates and (B: low wash and high wash1) show the average of 4 replicates. Error bars represent the standard deviation for all experiments with 3 or more replicates (B: standard wash, low wash and high wash2 and C and D: RT $20 \mathrm{~min}$ ) or the distance from the mean for experiments with two replicates (A, B: high wash1 and C and D: $4^{\circ} \mathrm{C} 20 \mathrm{~min}$ and $4^{\circ} \mathrm{C} 60 \mathrm{~min}$ ). 
In summary, no changes were made to the short bead beat protocol, because overnight elution with TE/SDS and incubation of chromatin and antibody with the beads for $20 \mathrm{~min}$ at RT are sufficient. The signal to noise ratio of the IP could be further improved by washing more stringently.

\section{Protein degradation during sonication}

We next examined how well the short bead beat protocol was performing using other TFs. When the stability of Abf1 was analyzed using the short bead beat protocol, a substantial amount of degradation was observed during cell lysis (Fig 8A, lanes 1-6). This degradation was even more pronounced after the sonication step (Fig 8A, lanes 2 and 5), with only $1 \%$ of the signal in the lane coming from intact Abf1. This means that the vast majority of Abf1 that was present during the IP was degraded, which can have detrimental effects on the ChIP efficiency. During the bead beat protocol, proteases are inhibited by addition of a tablet containing a cocktail of protease inhibitors. The substantial amount of Abf1 degradation can potentially be explained by insufficient inactivation of proteases by these protease inhibitors. To assess if proteases could be inhibited more efficiently, addition of four separate protease inhibitors during multiple steps in the protocol was tested. These protease inhibitors were aprotinin, pepstatin, leupeptin and PMSF (collectively abbreviated as APLP), which together inhibit a broad spectrum of proteases. With the addition of APLP, there is almost no additional degradation during cell lysis, and the degradation during sonication is also reduced (Fig 8A, lanes 7-12). As expected, besides reduced degradation of Abf1, there was also an increase in ChIP signal of Abf1 targets FCF1 and NHX1 when APLP is added (Fig 8B). This indicates that addition of APLP at multiple steps in the protocol rather than a protease inhibitor tablet at the beginning, offers better protection against proteases, and thus a stronger ChIP signal. Therefore, a revised version of the short bead beat protocol (version 2) is performed with the addition of APLP at multiple steps rather than a protease inhibitor tablet at the start.

After establishing the superior protection of Abf1 from proteases by APLP, we asked whether the stability of other proteins was also affected during the ChIP protocol. Therefore, the amount of degradation was determined for other TFs as well (Cbf1, Mcm1, Reb1 and Sum1), using the short bead beat (version 2) protocol. All four TFs were degraded during sonication to some extent, but especially the larger proteins, Reb1 (135 kDa) and Sum1 (162 kDa) were more severely affected compared to the smaller proteins Cbf1 (83 kDa) and Mcm1 (76 kDa, Fig 8C, compare lanes 7-12 and 20-25 with lanes 1-6 and 14-19). This indicates that large proteins are more sensitive to degradation during sonication, as expected and shown previously (Pchelintsev et al, 2016). The degradation could be caused by residual protease activity, the mechanical force during the sonication or a combination thereof.

\section{Increased formaldehyde concentration increases ChIP signal}

The last part of the protocol that was re-optimized was the cross-linking step (Fig 1, step II). It has been shown that for some TFs cross-linking efficiency can be improved by using a higher concentration of formaldehyde (Zaidi et al, 2017). An improved cross-linking efficiency means that with a similar time of cross-linking, more cross- 
A

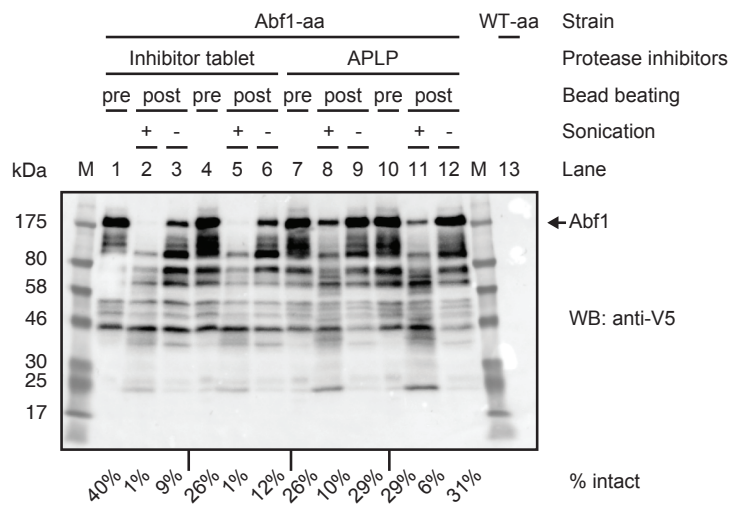

B

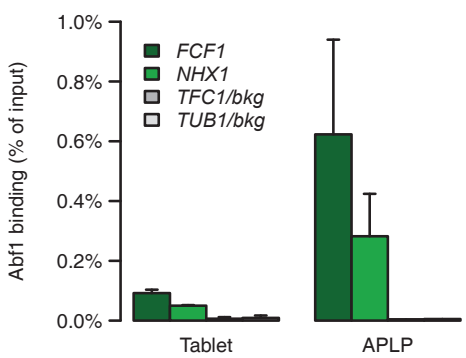

C

WT-

Cbf1-aa Mcm1-aa Reb1-aa Sum1-aa aa pre post pre post pre post pre post - $\pm \frac{- \pm}{- \pm \pm} \div \pm$

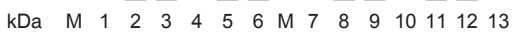
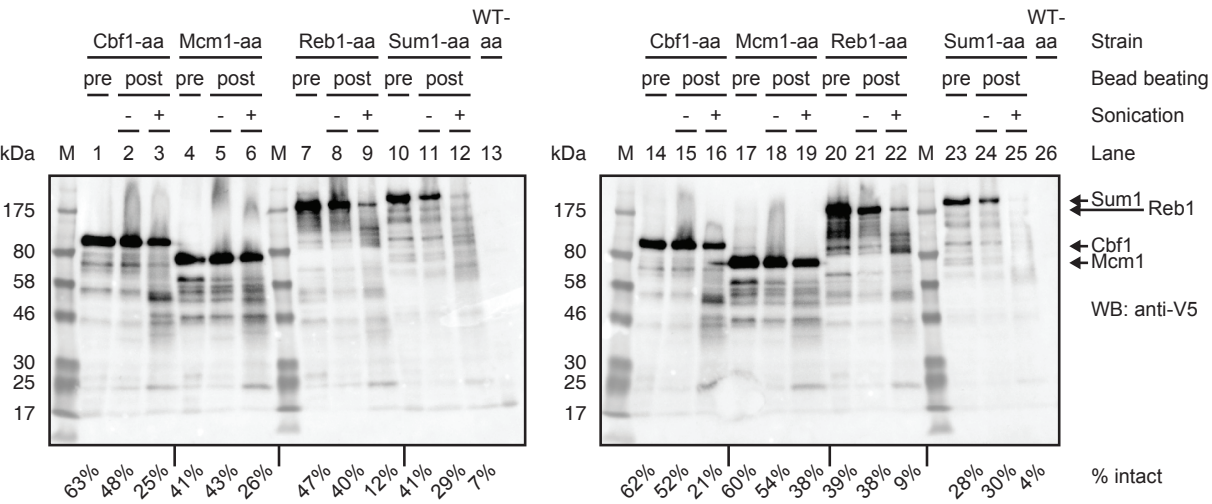

Figure 8. Addition of separate protease inhibitors partly prevents degradation during sonication (A) Western blot showing the presence of Abf1, which was obtained with either addition of a protease inhibitor tablet or addition of separate protease inhibitors (aprotinin, pepstatin, leupeptin and PMSF). Samples before bead beating, after sonication and before sonication are shown. (B) Percent of input DNA recovered of the samples shown in (A). Two replicates were used and the error bars show the distance from the mean. TFC1 and TUB1 were used as background controls (gray), FCF1 and NHX1 are Abf1 targets (Kasinathan et al, 2014, green) (C) Western blots showing presence of Cbf1, Mcm1, Reb1 and Sum1. Samples before bead beating, before sonication and after sonication are shown. All the blots shown were incubated with an antibody specific for V5. Tagged Abf1, Cbf1, Mcm1, Reb1 and Sum1 have a mass of approximately $125, \mathrm{kDa}, 83 \mathrm{kDa}, 76 \mathrm{kDa}, 135 \mathrm{kDa}$ and $162 \mathrm{kDa}$ respectively. The locations of these proteins on the blot are indicated on the right side of the corresponding blots. For all blots a crude lysate from a WT-aa strain was used as a negative control. Below all blots is indicated what percentage of the protein was still intact. This was calculated by dividing the signal from the intact protein (upper band) by the total signal in the lane (upper band + all lower bands).

links are formed between the protein of interest and DNA, which should result in an increased ChIP signal. To test this, Abf1-aa and Reb1-aa strains were cross-linked with $1 \%, 2 \%$ or $3 \%$ formaldehyde and processed with the short bead beat (version 2) protocol. Comparing the integrity of Abf1 treated with increasing amounts of formaldehyde indicates that a higher concentration of formaldehyde leads to less degradation during sonication (Fig 9A, compare lanes 1-3 with lanes 4-12). Whereas 
A

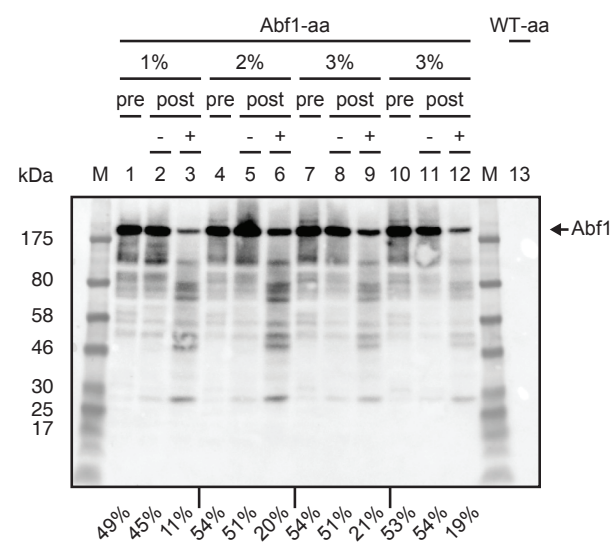

C

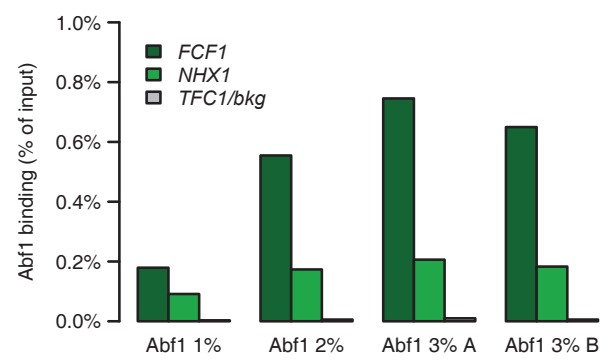

B $\begin{array}{llll}\frac{\text { Reb1-aa }}{1 \%} & \frac{2 \%}{\text { pre post }} \frac{3 \%}{\text { pre post pre post pre post }} \frac{3 \%}{\text { WT-aa }} & \begin{array}{l}\text { Strain } \\ \text { Formaldehyde }\end{array} \\ \text { Bead beating }\end{array}$

Sonication

$14 \overline{15} \overline{16}$ M $17 \overline{18} \overline{19} 20 \overline{21} \overline{22} 23 \overline{24} \overline{25}$ M $26 \quad$ Lane

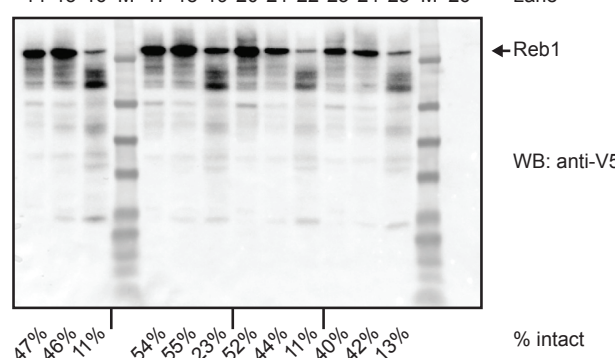

D

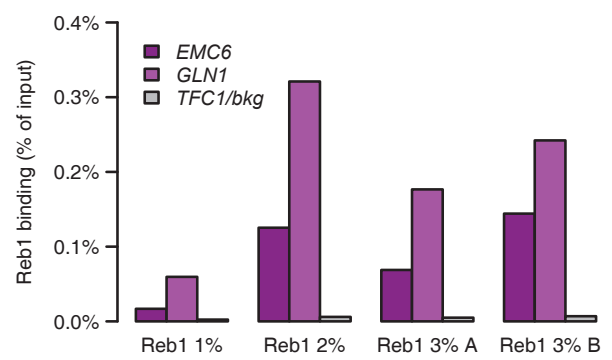

Figure 9. A higher percentage of formaldehyde leads to less degradation and higher ChIP signal (A and B) Western blots showing the presence of (A) Abf1 and (B) Reb1 with increasing concentrations of formaldehyde. Samples before bead beating, before sonication and after sonication are shown. For both blots a crude lysate from a WT-aa strain was used as a negative control. Tagged Abf1 and Reb1 have a mass of approximately $125 \mathrm{kDa}$ and $135 \mathrm{kDa}$, respectively. The location of these proteins on the blot is indicated on the right side of the corresponding blots. Both blots were incubated with an antibody specific for V5. Below the blots is indicated what percentage of the protein was still intact. This was calculated by dividing the signal from the intact protein (upper band) by the total signal in the lane (upper band + all lower bands). (C and D) Percent of input DNA recovered for Abf1 (C) and Reb1 (D), the samples are the same as shown in (A and B). Single replicates were used. TFC1 was used as a background control (gray), FCF1 and NHX1 are Abf1 targets (Kasinathan et al, 2014, green) and EMC6 and GLN1 are Reb1 targets (Kasinathan et al, 2014, purple). The 3\% formaldehyde samples differed only in the final concentration of Tris used for quenching. The 3\% A samples (lanes 7-9 and 20-22) were quenched with a final concentration $2.1 \mathrm{M}$ Tris and the $3 \% \mathrm{~B}$ samples (lanes 10-12 and 23-25) were quenched with a final concentration of $1.5 \mathrm{M}$ Tris.

for Abf1 a similar increase in stability is seen between $2 \%$ and $3 \%$ formaldehyde, for Reb1 this increase in stability was only observed with $2 \%$ formaldehyde (Fig 9B, compare lanes 17-19 with lanes 14-16 and 20-25). As expected, with an increased formaldehyde concentration, the ChIP signal of Abf1 also increased (Fig 9C). The ChIP signal of Reb1 increased with $2 \%$ and 3\% formaldehyde compared to the $1 \%$ formaldehyde ChIP signal, but was highest with $2 \%$ formaldehyde (Fig 9D). This suggests that for both proteins the ChIP signal can be improved by using higher concentrations of formaldehyde, although it has to be pointed out that these experiments were carried out using only single replicates. Note that due to a handling 
error, a similar amount of IP material was lost for the $1 \%$ and $2 \%$ Abf1 samples, which means that these signals would otherwise have been higher. In conclusion, a higher percentage of formaldehyde can increase the ChIP signal for both Abf1 and Reb1, not only by increasing cross-linking efficiency but likely also by preventing a part of the proteolytic degradation during sonication.

\section{An optimized ChIP protocol}

This study results in several improvements to a standard ChIP protocol (see chapter 4). The use of magnetic beads rather than agarose beads improves the speed and practicality of the IP. Using magnetic beads also leads to reduced background signal and a higher ChIP enrichment. Quenching with glycine is very inefficient at levels commonly used in ChIP protocols. Concentrated Tris is a much more efficient quencher. In addition, the method for cell lysis was optimized, since zymolyase treatment can degrade the protein of interest, likely by the presence of proteases. This step can be improved by mechanically disrupting the cells using bead beating, which causes less degradation and a strongly increased ChIP signal compared to zymolyase treated samples. The bead beating protocol can be further improved by removing the last centrifugation step, leading to an easier and quicker protocol. However, care has to be taken that the protein of interest does not rebind DNA during the subsequent incubation steps. There is a substantial amount of degradation taking place during cell lysis and sonication as well. Addition of a more optimal mix of protease inhibitors at several steps during the protocol, rather than a tablet containing a mix of inhibitors added only once, can alleviate part of this degradation. Nonetheless, even with these inhibitors there is still a substantial amount of degradation observed during sonication, especially for very large proteins. Cross-linking with a higher concentration of formaldehyde may reduce the extent of this degradation for some proteins.

Based on the results shown here we recommend the following protocol: 1) crosslinking the cells with $2 \%$ formaldehyde and 2) quenching them with an appropriate concentration of Tris (1.5M). 3) Not lysing the cells using zymolyase but lysing them mechanically, using for example a bead beater. 4) Using a shorter version of the bead beat protocol, but taking care that the protein of interest does not bind DNA in vitro during the protocol. 5) Adding the protease inhibitors aprotinin, pepstatin, leupeptin and PMSF during cell lysis and subsequent steps. 6) Using magnetic beads for the IP steps, as this allows for a faster and more reproducible IP, with stronger ChIP signal and less background. The complete optimized protocol is presented in detail in chapter 4 .

\section{Discussion}

\section{Extent of cross-linking affects downstream steps}

We optimized multiple steps of the protocol, and one of the steps that varies strongly between ChIP protocols is the time of cross-linking. In general, longer cross-linking times lead to stronger ChIP signals. Increased crosslinking can result in stronger 
signal in two ways. Firstly, this increase in signal can be explained by proteins that bind DNA during cross-linking (Poorey et al, 2013). These additional protein-DNA interactions get fixed by the formaldehyde, which will be picked up as increased signal. Alternatively, cross-linking of the protein of interest to DNA may not be very efficient and with longer incubation times there is an increased chance of the protein being cross-linked (Zaidi et al, 2017). Either one or both may be happening, depending on how efficiently the protein studied can be cross-linked to DNA.

Considering that longer cross-linking times lead to stronger ChIP signals, it might be tempting to increase the cross-linking time as long as possible. However, in addition to crosslinking protein-DNA interactions, formaldehyde can also crosslink proteinprotein interactions (Sutherland et al, 2008). Prolonged crosslinking can cause extensive crosslinking of proteins to other proteins, and large protein aggregates could form in the cells. Therefore, the extent of cross-linking can have a significant effect on downstream processing steps. For example, during cell lysis there is a centrifugation step that clears cell debris from the chromatin. With increased cross-linking times a large part of the chromatin may co-precipitate with the cell debris, depending on the centrifugation speed. This could lead to reduced yields if the majority of the chromatin is subsequently discarded with the cell debris. The efficiency of sonication also changes with cross-linking time. Increased cross-linking leads to stiffer and more rigid DNA, which may be more resistant to shearing by sonication. More sonication cycles may be needed to sufficiently fragment the DNA and this could have detrimental effects on the stability of the protein of interest.

Apart from technical issues, long cross-linking times also increase well-known ChIP artefacts. Highly expressed genes tend to show as peaks in ChIP data (Park et al, 2013; Teytelman et al, 2013) and this is more prevalent with increased crosslinking times (Baranello et al, 2015). This artefactual signal might stem from the open chromatin that is present at these loci, or from crosslinking of the protein of interest to other DNA-bound factors. Additionally, with longer cross-linking times we also noticed an increase in background signal and of the non-tagged controls. Thus, longer cross-linking times may lead to increased signal, but not necessarily to an increased enrichment over background or biologically meaningful signal.

An alternative to longer cross-linking times is increasing the concentration of formaldehyde. A higher concentration of formaldehyde will increase the efficiency of cross-linking, which could increase the ChIP signal if this efficiency is ratelimiting. However, it may also increase ChIP artefacts, depending on their cause. In our optimized protocol we use 5 minutes cross-linking with $2 \%$ formaldehyde as a good balance between a relatively short cross-linking time, but still long enough to get clear signal. Note, however, that optimal cross-linking conditions may differ depending on the proteins studied.

\section{Proper quenching is crucial for quantitative protocols}

The results presented here show that glycine is an inefficient quencher (Fig 3) (Sutherland et al, 2008; Zaidi et al, 2017). Interestingly, most published ChIP protocols 
that cross-link with formaldehyde, quench with glycine at sub-stoichiometric concentrations (Kuo \& Allis, 1999; Acevedo et al, 2007; Rhee \& Pugh, 2011; Poorey et al, 2013; He et al, 2015; Skene \& Henikoff, 2015; Gutin et al, 2018). How is it possible that such an inefficient quenching agent became the quencher of choice for virtually all ChIP protocols? A likely explanation lies in its inefficiency as a quencher, which is linked to the stronger ChIP signals that can be observed with longer cross-linking times. Inefficient quenching effectively means that the cross-linking time is increased and thus that there is increased ChIP signal. Considering this, it is easy to imagine that glycine was preferred as a quencher, since it would result in a higher signal. An alternative explanation could be that during the development of the early ChIP protocols inactivation of formaldehyde was only desirable to discard the waste in a safe way. If the only concern is to inactivate formaldehyde for waste routing purposes, it is apparently less critical to efficiently and rapidly quench formaldehyde, and thus to efficiently stop the cross-linking reaction.

Considering that glycine does not quench and that the first ChIP protocols did not include a quenching step (Solomon et al, 1988; Dedon et al, 1991), the question arises as to whether it is really necessary to quench at all. In fact it may be better not to quench and to be aware that the cross-linking reaction is still proceeding, rather than quenching inefficiently and assuming that the reaction has stopped. If it is assumed that the reaction is efficiently stopped, quenching with glycine gives a false sense of security and this can have detrimental effects on the reproducibility. For example, this can happen when some samples are left on ice after quenching while others are being processed, because it is assumed that the cross-linking has stopped. In fact, cross-linking is still proceeding, thus the effective cross-linking time will be longer than intended. Even worse, the extent of cross-linking in this case will vary between samples or replicates. This can be a major source of variation and make the protocol irreproducible.

The experiments here show that in contrast to glycine, Tris is an efficient quencher (Fig 3), as has indeed been proposed previously (Sutherland et al, 2008). Some have raised concerns that quenching with Tris may revert the protein-DNA cross-links (Hoffman et al, 2015; Zaidi et al, 2017). The results here show no evidence of de-crosslinking when using short Tris incubation times, as has been shown by others for long incubation times without heating (Sutherland et al, 2008; Kawashima et al, 2014). An alternative to quenching with Tris is using highly concentrated (2.93 M) glycine solutions (Zaidi et al, 2017). While it is a more efficient way to quench compared to sub stoichiometric glycine solutions, it also involves concentrating the samples using centrifugation. This concentrating of the samples potentially stresses the cells. This, in combination with the impracticality of quenching with $2.93 \mathrm{M}$ glycine, which involves addition of $440 \mathrm{ml} 3 \mathrm{M}$ glycine to $10 \mathrm{ml}$ of cells, makes quenching with Tris preferable.

In conclusion, quenching with sub stoichiometric levels of glycine is very inefficient and a source of variation in ChIP protocols. Quenching with Tris is much more efficient, quicker, does not require an extra centrifugation step and does not de- 
cross-link the sample. Together this makes Tris a better quencher than glycine. The quenching step that we now incorporated in the optimized protocol facilitates higher reproducibility of the procedure.

\section{Strategies that reduce protein degradation increase ChIP signal}

Many steps of the ChIP protocol can have an effect on the stability of the protein studied, including the extent of cross-linking, cell lysis, shearing of the DNA and the IP step. The steps that have the biggest influence on the stability of the proteins studied are cell lysis and sonication. In particular, cell lysis with zymolyase caused a significant decrease in detectable levels of the protein of interest in the samples (Fig 4). This is likely caused by proteases that are present in zymolyase preparations. A significant part of this degradation can be prevented by lysing cells mechanically using bead beating with appropriate protease inhibitors (Fig 5). Although it was not tested here, using additional protease inhibitors and more extensive washes may also prevent part of the degradation caused by zymolyase treatment.

Even when cells are used that do not have a cell wall, cell lysis is an important step. Differences in lysis efficiency between different mammalian cell types are a major source of variation in ChIP protocols (Arrigoni et al, 2016). A lysis strategy that is efficient across different cell types can greatly reduce variation between protocols (Arrigoni et al, 2016), which highlights the importance of the reproducibility of this step.

The second step that causes significant degradation is sonication. Using more efficient protease inhibitors reduces the amount of degradation during this step, but cannot prevent all degradation (Fig 8). This indicates that the mechanical force of the sonication also degrades the protein of interest. Large proteins are more sensitive to this degradation compared to smaller proteins (Pchelintsev et al, 2016). Thus, sonication may be further optimized to reduce the amount of degradation, but care has to be taken that the fragmentation of the DNA is still suited for downstream applications in terms of reproducibility and fragment size.

Even after the optimizations described here, there is still a substantial amount of degradation of most proteins that were studied. Future efforts to further reduce the extent of this degradation likely will increase the ChIP signal even more. For example, as an alternative to sonication, the DNA can also be fragmented enzymatically using DNases such as MNase (Skene \& Henikoff, 2015; Arrigoni et al, 2018; Gutin et al, 2018). This approach could prevent the degradation that was observed during sonication and may subsequently increase the ChIP signal. Especially for large proteins this approach may be beneficial.

\section{Limitations and outlook}

Although the optimizations carried out here greatly increase the specific ChIP signal and reduce variation, there are still some aspects that have to be taken into account before extrapolation to other experiments. This protocol was optimized using yeast. Most likely the optimizations are applicable for ChIP protocols using other 
organisms as well, but this has not been tested. In addition, all optimizations were performed using V5-tagged strains and an antibody that specifically recognizes this tag. Some conditions that were found to be optimal here might be specific for this antibody and should be verified when performing ChIP using other antibodies.

The optimizations described here were not all carried out with identical numbers of replicates and with the same proteins. In addition, even though this protocol has been extensively optimized, there is still some variation between replicates. This is clearly evident in Fig 3 and 7, where there is a difference in ChIP signal between ChIPs performed using the same conditions on different days. This highlights the need for the additional optimization steps carried out afterwards. Still, the protocol may yet be further improved. For example, optimizations that additionally reduce the number of steps or pool the samples early in the protocol could be beneficial to further reduce the variation between replicates. An example of such a strategy is barcoding of the DNA while it is bound to the beads (Lara-Astiaso et al, 2014; Schmidl et al, 2015; Wallerman et al, 2015; Gutin et al, 2018) or fragmentation and barcoding of DNA while still in the nucleus (Arrigoni et al, 2018). Alternatively, many orthogonal method exist that quantify protein-DNA binding, which may be used if no clear ChIP signal can be obtained (Galas \& Schmitz, 1978; Garner \& Revzin, 1981; Siebenlist \& Gilbert, 1980; Siebenlist et al, 1980; Smith, 1985; Nick \& Gilbert, 1985; Ellington \& Szostak, 1990; Tuerk \& Gold, 1990; Wang \& Reed, 1993; Yin et al, 1995; van Steensel \& Henikoff, 2000; Bulyk et al, 2001; Schmid et al, 2004; Cremazy et al, 2005; Lushnikov et al, 2006; Maerkl \& Quake, 2007; Zentner et al, 2015; Skene \& Henikoff, 2017). Using the short bead beat protocol, one protein (Cbf1) was able to re-bind DNA during the IP step of the protocol (Fig 6). Depending on the affinity of the protein for DNA, more stringent washes may remove such binding.

Although many labs have undertaken efforts to optimize the ChIP protocol, it remains a challenging technique. Many different protocols exist and most will not be ideal for the majority of proteins or conditions studied. Since many proteins of interest will have a different set of optimal ChIP parameters, ideally every ChIP protocol should be optimized for each protein separately. Nevertheless, this work highlights the steps that are crucial for accurate ChIP quantifications. The final protocol, described in full detail in the materials and methods section, is superior to previous standard protocols and will form an excellent starting point for many studies employing ChIP.

\section{Materials and Methods}

Here, the general methods used are described. In the supplemental methods the differences from the general methods are highlighted for each figure separately as well as 3 supplemental tables that describe the strains, primers and reagents that were used in this study. The full, optimized ChIP protocol is described in chapter 4 . 


\section{Strains}

All the Saccharomyces cerevisiae strains that were used are anchor away strains (Haruki et al, 2008) that were re-made in the BY4742 background, as is described in (de Jonge et al, 2017). The anchor away tag consists of the FRB domain from the mammalian target of rapamycin (mTOR), a yeast enhanced green fluorescent protein (yEGFP) and three times a V5 tag (3V5). In addition to the Cbf1-aa strain, an additional strain was made where Cha4 was tagged with a 3 V5 tag as an internal control. The Cbf1-aa strain with a $3 \mathrm{~V} 5$ tagged Cha4 was used in Fig 3A, 3B and 5. The genotypes of all strains are shown in Table 1.

\section{Growth conditions}

The growth conditions were similar for all the experiments performed. Strains were streaked directly from $-80^{\circ} \mathrm{C}$ stocks on appropriate selective plates (YPD + Nourseothricin for the WT-aa strain and YPD + Hygromycin + Nourseothricin for anchor away strains) and grown for 3 days at $30^{\circ} \mathrm{C}$. Liquid pre-cultures were inoculated in the morning, diluted at the end of the day and grown overnight at $30^{\circ} \mathrm{C}$ with shaking $(230 \mathrm{rpm})$ in synthetic complete (SC) medium: $2 \mathrm{~g} / 1$ dropout mix complete and $6.71 \mathrm{~g} / 1$ yeast nitrogen base without amino acids, carbohydrate \& w/ AS (YNB) from US Biologicals (Swampscott, USA) with 2\% D-glucose. The next morning, cultures were diluted in $100 \mathrm{ml}$ to an OD of 0.2 (WPA Biowave CO8000 Cell Density Meter) and grown at $30^{\circ} \mathrm{C}, 230 \mathrm{rpm}$ for 2 doublings until an OD of 0.8 was reached, which corresponds to about $2^{*} 10^{7}$ cells per $\mathrm{ml}$.

\section{Cross-linking and quenching}

When the cultures reached an OD of 0.8 , formaldehyde was added to a final concentration of $1 \%$, by adding 2.7 or $2.8 \mathrm{ml}$ of $37 \%$ formaldehyde (Sigma-Aldrich \#252549) to a $100 \mathrm{ml}$ culture (Fig 2E, 3, 6, 8 and 9). When $250 \mathrm{ml}$ cultures were used (Fig 2A-D, 4, 5 and 7), $6.8 \mathrm{ml} 37 \%$ formaldehyde was added. A final concentration of $1 \%$ was used for all experiments except those shown in Fig 9, where some cultures were cross-linked with a final concentration of $2 \%(5.7 \mathrm{ml}$ of $37 \%$ formaldehyde) or $3 \%$ ( $8.8 \mathrm{ml}$ of $37 \%$ formaldehyde). To accurately control the time of cross-linking, the addition of formaldehyde and quenching agent was performed outside the shaker, on a heated stir plate (IKA C-MAG HS 7) set to $30^{\circ} \mathrm{C}$ and $\sim 250 \mathrm{rpm}$, with a magnetic stir bar. An exception was made for the $250 \mathrm{ml}$ cultures that were grown as a big batch. For these cultures, the formaldehyde and quencher were added and mixed in the incubator (Fig 2A-D, 4, 5 and 7).

Quenching was performed using either glycine or Tris (tris(hydroxymethyl) aminomethane). Glycine was added to a final concentration of $125 \mathrm{mM}$ by adding $5.1 \mathrm{ml}$ of $2.5 \mathrm{M}$ glycine to a $100 \mathrm{ml}$ culture, except when otherwise indicated (Fig 3B, $250 \mathrm{mM}$ : addition of $10.3 \mathrm{ml} 2.5 \mathrm{M}$ glycine). When $250 \mathrm{ml}$ cultures were used, $12.8 \mathrm{ml}$ of $2.5 \mathrm{M}$ glycine was added. Glycine quenching was used for all experiments shown in Fig 2, 3 and 4. When the samples were quenched using Tris, this was done with a final concentration of $500 \mathrm{mM}$ (Fig 5 and 7), $750 \mathrm{mM}$ (Fig 3C and 3D, 6, 8 and 9: 1\% samples), 1.5M (Fig 9: 2\% and 3\% B samples) or 2.0M (Fig 9, 3\% A samples) Tris pH 8.0. Cells that were grown in $250 \mathrm{ml}$ cultures were quenched using $500 \mathrm{mM}$ Tris by 
adding $32.1 \mathrm{ml} 4.5 \mathrm{M}$ Tris $\mathrm{pH} 8.0$ (Fig 5 and 7) or using $750 \mathrm{mM}$ Tris by adding 51.4 $\mathrm{ml} 4.5 \mathrm{M}$ Tris $\mathrm{pH} 8.0$ (Fig 8A and $\mathbf{8 B}$ ). When $100 \mathrm{ml}$ cultures were quenched with $750 \mathrm{mM}$ Tris, $20.6 \mathrm{ml} 4.5 \mathrm{M}$ Tris $\mathrm{pH} 8.0$ was added (Fig 6, 8C and 9). The cultures that were quenched with $1.5 \mathrm{M}$ Tris after cross-linking with $2 \%$ or $3 \%$ formaldehyde were quenched by adding $52.9 \mathrm{ml}$ or $54.4 \mathrm{ml}$ of $4.5 \mathrm{M}$ Tris $\mathrm{pH} 8.0$, respectively (Fig 9). For the $3 \%$ formaldehyde cross-linked samples that were quenched with $2.0 \mathrm{M}$ Tris, $87.1 \mathrm{ml}$ 4.5M Tris pH 8.0 was added (Fig 9). Quenching with glycine was done for 5 minutes and with Tris for 1 minute, unless otherwise stated (Fig 3).

\section{Harvesting cross-linked cells}

$100 \mathrm{ml}$ cultures were harvested after quenching by splitting the culture over either 2 (glycine quenched), 3 (750 mM and 1.5M Tris quenched) or 4 (2.0M Tris quenched) $50 \mathrm{ml}$ tubes. The cells were pelleted by centrifugation at $3220 \mathrm{~g}(4000 \mathrm{rpm})$ in an Eppendorf $5810 \mathrm{R}$ centrifuge at $4^{\circ} \mathrm{C}$ for 3 minutes. The supernatant was discarded, the pellet from the first tube was resuspended in $10 \mathrm{ml}$ TBS $(150 \mathrm{mM} \mathrm{NaCl}, 10 \mathrm{mM}$ Tris pH 7.5) and subsequently combined with the other pellets that came from the same culture. The cells were pelleted again at $3220 \mathrm{~g}$ for 3 minutes at $4^{\circ} \mathrm{C}$, resuspended in $1 \mathrm{ml} \mathrm{MQ}$ and transferred to a $2 \mathrm{ml}$ safe-lock tube. The cells were centrifuged at $3381 \mathrm{~g}(6000 \mathrm{rpm})$ in an Eppendorf 5424 centrifuge at room temperature (RT) for 20 seconds. The supernatant was discarded and the samples were subsequently snap frozen and stored at $-80^{\circ} \mathrm{C}$.

$250 \mathrm{ml}$ cultures were harvested slightly differently. After quenching, the cells were centrifuged in $500 \mathrm{ml}$ centrifuge bottles at $2831 \mathrm{~g}$ (4000 rpm) in a Beckman Coulter Avanti J-E 369003 centrifuge for 3 minutes at $4^{\circ} \mathrm{C}$. The supernatant was discarded and the pellets were pooled per 2 pellets by resuspending the first pellet in $40 \mathrm{ml}$ ice-cold TBS (150 mM NaCl, $10 \mathrm{mM}$ Tris $\mathrm{pH}$ 7.5), combining this with another pellet and transferring them to a $50 \mathrm{ml}$ tube. By combining 2 pellets, the tube contains the equivalent of a $500 \mathrm{ml}$ culture. The cells were next pelleted by centrifugation at $3220 \mathrm{~g}(4000 \mathrm{rpm})$ in an Eppendorf $5810 \mathrm{R}$ centrifuge at $4^{\circ} \mathrm{C}$ for 3 minutes. The supernatant was discarded, the cells were resuspended in $4.5 \mathrm{ml}$ ice-cold MQ and divided in $1 \mathrm{ml}$ aliquots over $5 \times 2 \mathrm{ml}$ safe-lock tubes. After centrifuging at $3381 \mathrm{~g}$ in an Eppendorf 5424 centrifuge for 20 seconds at RT, the supernatant was discarded and the cells snap frozen in liquid nitrogen. Each pellet now contains the equivalent of a $100 \mathrm{ml}$ culture.

\section{Nuclear depletion}

Nuclear depletion was achieved by using the anchor away system (Haruki et al, 2008). The nuclear depletion was induced by addition of $2 \mathrm{mM}$ DMSO dissolved rapamycin to the cultures to a final concentration of $7.5 \mu \mathrm{M}$. The rapamycin was added in such a way that the samples were ready for harvesting at $\mathrm{OD}=0.8$. For 60 and 15 minutes depletion of Cbf1 (Fig 6A and 6B), $367.4 \mu \mathrm{l}$ of $2 \mathrm{mM}$ rapamycin was added to a $100 \mathrm{ml}$ culture at $\mathrm{OD}=0.52$ and 0.72 respectively. In addition, $367.4 \mu \mathrm{l}$ of $\mathrm{DMSO}$ was added to the non-depleted control at $\mathrm{OD}=0.52$. For nuclear depletion of Abf1 and Reb1 (Fig 6E and 6F) $758 \mu \mathrm{l}$ and $750 \mu \mathrm{l} 2 \mathrm{mM}$ rapamycin was added to 215 $\mathrm{ml}$ and $200 \mathrm{ml}$ cultures at $\mathrm{OD}=0.56$, respectively. For both Abf1 and Reb1 the same 
amount of DMSO (758 $\mu \mathrm{l}$ and $750 \mu \mathrm{l}$ respectively) was added to the non-depleted controls at $\mathrm{OD}=0.56$.

\section{Fluorescence microscopy}

Fluorescence microscopy was used to assess the degree of Cbf1 depletion from the nucleus. Cbf1, like all other TFs used in this study, was tagged with yEGFP, which allows monitoring of nuclear localization. To assess the degree of nuclear depletion of Cbf1-aa, cells were grown and Cbf1 was depleted from the nucleus as detailed in the previous sections. After depletion, the cells were fixed using methanol as described previously (Haruki et al, 2008). At OD $=0.8$, after either 60 minutes of $\mathrm{DMSO}$, or 60 minutes or 15 minutes of rapamycin treatment, $1 \mathrm{ml}$ aliquots of the cultures were transferred to $1.5 \mathrm{ml}$ Eppendorf tubes. The cells were collected by centrifugation at 3381g (6000 rpm) in an Eppendorf 5424 centrifuge for 1 minute at RT. The supernatant was discarded and the cells were fixed in $1 \mathrm{ml}-20^{\circ} \mathrm{C} 100 \%$ methanol for 6 minutes. The cells were again pelleted at 3381g, and rehydrated in PBS containing $0.2 \%$ Tween-20 for 5 minutes. After centrifuging the cells at $3381 \mathrm{~g}$, the cells were resuspended in $100 \mu \mathrm{l}$ PBS. $1.5 \mu \mathrm{l}$ was used for imaging, by combining this with $1.5 \mu \mathrm{l}$ of $1 \%$ agarose on a microscopy slide $(50 \times 24 \mathrm{~mm})$ and quickly covering the cells with a coverslip $(22 \times 22 \mathrm{~mm})$.

The cells were imaged using a DeltaVision Elite high resolution microscope, with an Olympus 100X/1.40 oil objective and a CoolSNAP HQ2-ICX285 camera operating at $-25^{\circ} \mathrm{C}$. The resolution was set to $512 \times 512$ pixels and binning to $2 \times 2$. To visualize GFP, EX and EM filters were set to FITC, the ND filter was set to $100 \%$ and an exposure time of 0.1 seconds was used. For each image, a z-stack of 11 images was taken with optical sectioning set to $1 \mu \mathrm{m}$ and the images were deconvolved using the Resolve3D softWoRx software. A projection was made from the deconvolved images using the maximum intensity with the softWorx software. These projections are shown in Fig 6B. All GFP images shown here were set to have the same brightness and contrast levels.

Along with each z-stack of GFP images, a single reference image was taken. This was achieved by setting the EX filter to POL, the EM filter to BLANK, the ND filter to $100 \%$ and by using an exposure time of 0.01 seconds. The reference images were processed as follows. Two blank images were taken on the same microscope using the same settings without any cells in the view. The average signal of these two blank images was calculated and from this blank image a value of 100 was subtracted using the subtract function in the ImageJ software (Schneider et al, 2012). Subsequently, this reduced blank image was subtracted from each reference image using the image calculator function in ImageJ. The brightness and contrast values were then set to the same levels for all reference images.

\section{Chromatin isolation using zymolyase}

Chromatin was isolated using zymolyase for the experiments shown in Fig 2, 3, 4, 5A and 5C. To isolate the chromatin, each cell pellet, which is equivalent to a $100 \mathrm{ml}$ culture of cells $\mathrm{OD}=0.8$, was resuspended in $1 \mathrm{ml}$ buffer $\mathrm{Z}$ ( $1 \mathrm{M}$ sorbitol, 
$50 \mathrm{mM}$ Tris $\mathrm{pH} 7.5)$ to change buffer. The cells were pelleted by centrifugation at $15871 \mathrm{~g}$ (13000 rpm) for 30 seconds in an Eppendorf 5424 centrifuge at RT. The cells were then resuspended in $1 \mathrm{ml}$ of buffer $\mathrm{Z}$ containing $10 \mathrm{mM} \beta$-mercaptoethanol and $10 \mathrm{mg} / \mathrm{ml}$ zymolyase (zymolyase 20T MP biomedical \#08320921) and incubated in a rotating wheel for 10 minutes (unless stated otherwise) to create spheroplasts. For some samples shown in $4 \mathrm{~B}$ and $4 \mathrm{C}$ protease inhibitors were added at this step (one tablet of Roche EDTA-free cOmplete protease inhibitor cocktail (\#11873580001) per $10 \mathrm{ml}$ buffer $\mathrm{Z}$ containing $\beta$-mercaptoethanol and zymolyase). The cells were pelleted by centrifugation at $15871 \mathrm{~g}$ for 10 seconds at room temperature and each pellet was washed with ice-cold buffer $\mathrm{Z}$ by inverting the tubes. The centrifugation and wash were repeated once. For some samples shown in $4 \mathrm{~B}$ and $4 \mathrm{C}$, protease inhibitors were added at this wash step as well (one tablet Roche cOmplete protease inhibitor cocktail (\#11836145001) per $10 \mathrm{ml}$ buffer Z). The spheroplasts were carefully resuspended in $550 \mu \mathrm{l}$ FA lysis buffer (50 mM HEPES-KOH pH 7.5, $150 \mathrm{mM} \mathrm{NaCl}, 1$ mM EDTA pH 8.0, 1\% Triton X-100, 0.1\% Na-deoxycholate, 0.1\% SDS) with protease inhibitors (1 tablet Roche cOmplete protease inhibitor cocktail per $25 \mathrm{ml}$ of FA lysis buffer) and split over 2x $1.5 \mathrm{ml}$ bioruptor pico microtubes (Diagenode). The cells were lysed and the chromatin was sheared in a bioruptor pico sonicator (Diagenode) by sonicating the samples for 3 or 4 cycles, 30 seconds on / 30 seconds off. The cell debris and unfragmented chromatin was subsequently pelleted by centrifugation at $21130 \mathrm{~g}(15000 \mathrm{rpm})$ in an Eppendorf $5424 \mathrm{R}$ centrifuge for 20 minutes at $4^{\circ} \mathrm{C}$. The 2 supernatants, containing the fragmented chromatin, of the samples that were split before sonication, were then combined again in a $1.5 \mathrm{ml}$ Eppendorf tube. The chromatin was snap frozen in liquid nitrogen and stored at $-80^{\circ} \mathrm{C}$, except for a $5 \mu \mathrm{l}$ aliquot that was taken to assess the extent of fragmentation of the DNA.

\section{Chromatin isolation using the full bead beating protocol}

Chromatin was isolated using the full bead beating protocol for a few samples shown in Fig 5. To isolate the chromatin, each cell pellet, which is equivalent to a $100 \mathrm{ml}$ culture of cells OD $=0.8$, was resuspended in $700 \mu \mathrm{l}$ of FA lysis buffer (50 mM HEPES-KOH pH 7.5, 150 mM NaCl, 1 mM EDTA pH 8.0, 1\% Triton X-100, $0.1 \%$ Na-deoxycholate, $0.1 \%$ SDS containing protease inhibitors (1 tablet Roche cOmplete protease inhibitor cocktail (\#11836145001) per $25 \mathrm{ml}$ of FA lysis buffer). The resuspended cells were subsequently added to a 2-ml screw-cap tube containing $500 \mu \mathrm{l}$ of zirconium/silica beads $0.5 \mathrm{~mm}$ (BioSpec Products, \#11079105z). If needed, the tubes were closed and inverted once to remove air trapped in the beads. The tube was then filled completely with FA lysis buffer, to keep as little air as possible. The tubes were then bead beated 7 times for 3 minutes in a genie disruptor. The samples were put one ice for 1 minute in between each run. During the bead beating, $15 \mathrm{ml}$ tubes were prepared containing $1 \mathrm{ml}$ pipette tips with the end cut off. After bead beating, the lysate was recovered by burning a hole in the top and bottom of the screw-cap tubes using a hot 23G needle and placing them on top of the pipette tip in the $15 \mathrm{ml}$ tube. This combination was then centrifuged at $201 \mathrm{~g}$ (1000 rpm in an Eppendorf $5810 \mathrm{R}$ ) at $4^{\circ} \mathrm{C}$ for 1 minute and the lysate was transferred to a $2-\mathrm{ml} \mathrm{screw-}$ cap tube. To remove the majority of unbroken cells and cell debris, the lysate was centrifuged at $1503 \mathrm{~g}(4000 \mathrm{rpm})$ for 2 minutes in an Eppendorf $5424 \mathrm{R}$ centrifuge at 
$4^{\circ} \mathrm{C}$. The supernatant was transferred to a new 2-ml Eppendorf tube and centrifuged at $18407 \mathrm{~g}(14000 \mathrm{rpm})$ for 15 minutes in an Eppendorf $5424 \mathrm{R}$ centrifuge at $4^{\circ} \mathrm{C}$. The pellet (chromatin extract, CE) and the supernatant (whole cell lysate, WCL) were then treated differently.

\section{Chromatin extract (CE)}

The CE was washed using $1.5 \mathrm{ml}$ FA lysis buffer by resuspending the pellet using a $23 \mathrm{G}$ needle and incubating in a rotating wheel at $4^{\circ} \mathrm{C}$ for 30 minutes. Chromatin was pelleted again by centrifugation at $18407 \mathrm{~g}(14000 \mathrm{rpm})$ for 15 minutes in an Eppendorf $5424 \mathrm{R}$ centrifuge at $4^{\circ} \mathrm{C}$. For Fig 5A, this pellet was washed again once with $700 \mu \mathrm{FA}$ lysis buffer without resuspending the pellet, but this step was skipped for the other experiment (Fig 5B-5D). The pellet was then resuspended in $600 \mu \mathrm{FA}$ lysis buffer using a syringe and a 23G needle and split over 2x $1.5 \mathrm{ml}$ bioruptor pico microtubes (Diagenode). The chromatin was sheared in a bioruptor pico sonicator (Diagenode) by sonicating the samples for 3 cycles, 30 seconds on / 30 seconds off. The chromatin was snap frozen in liquid nitrogen and stored at $-80^{\circ} \mathrm{C}$, except for a 5 $\mu \mathrm{l}$ aliquot that was taken to assess the extent of fragmentation of the DNA.

Whole cell lysate (WCL)

For Fig 5B-D, the WCL was fragmented as well by sonicating for 3 cycles 30 seconds on / 30 seconds off in $15 \mathrm{ml}$ bioruptor pico tubes containing $300 \mu \mathrm{l}$ of sonication beads (Diagenode). After sonication, the sample was transferred a $2 \mathrm{ml}$ Eppendorf tube and the unfragmented chromatin was subsequently pelleted by centrifugation at $18407 \mathrm{~g}(14000 \mathrm{rpm})$ in an Eppendorf $5424 \mathrm{R}$ centrifuge for 20 minutes at $4^{\circ} \mathrm{C}$. The fragmented chromatin was transferred to a $2 \mathrm{ml}$ Eppendorf tube, snap frozen in liquid nitrogen and stored at $-80^{\circ} \mathrm{C}$, except for a $20 \mu \mathrm{l}$ aliquot that was taken to assess the extent of fragmentation of the DNA.

\section{Chromatin isolation using the short bead beating protocol}

Chromatin was isolated using the short bead beating protocol for the experiments shown in Fig 5B-5D, 6, 7, 8 and 9. To isolate the chromatin, each cell pellet, which is equivalent to a $100 \mathrm{ml}$ culture of cells $\mathrm{OD}=0.8$, was resuspended in $\sim 700-900 \mu \mathrm{l}$ of FA lysis buffer (50 mM HEPES-KOH pH 7.5, $150 \mathrm{mM} \mathrm{NaCl}, 1 \mathrm{mM}$ EDTA pH 8.0, $1 \%$ Triton X-100, 0.1\% Na-deoxycholate, $0.1 \%$ SDS) containing protease inhibitors: either 1 tablet Roche cOmplete protease inhibitor cocktail (\#11836145001) per $25 \mathrm{ml}$ of FA lysis buffer or $30 \mu \mathrm{l}$ aprotinin (Sigma-Aldrich: \#A6279), $1 \mu 1$ leupeptin (SigmaAldrich \#L2884, $1 \mathrm{mg} / \mathrm{ml}$ in MQ), 1 ul pepstatin A (Sigma-Aldrich \#P4265: $1 \mathrm{mg} / \mathrm{ml}$ in 100\% Methanol) and $15 \mu \mathrm{l}$ PMSF (Sigma-Aldrich: \#P7626, $200 \mathrm{mM}$ in isopropanol) per milliliter of FA lysis buffer. The resuspended cells were subsequently added to a $2 \mathrm{ml} \mathrm{screw-cap} \mathrm{tube} \mathrm{containing} 500 \mu \mathrm{l}$ of zirconium/silica beads $0.5 \mathrm{~mm}$ (BioSpec Products, \#11079105z). If needed, the tubes were closed and inverted once to remove air trapped in the beads. The tubes were then filled completely with FA lysis buffer to remove as much air as possible. The tubes were bead beated 7 times in a genie disruptor for 3 minutes. The samples were put one ice for either 1 or 3 minutes in between each run, depending on the number of samples that were processed at the same time. During the bead beating, $15 \mathrm{ml}$ tubes were prepared containing $1 \mathrm{ml}$ 
pipette tips with the end cut off. After bead beating, the lysate was recovered by burning a hole in the top and bottom of the screw-cap tubes using a 23G needle and placing them on top of the pipette tip in the $15 \mathrm{ml}$ tube. This combination was then centrifuged at $201 \mathrm{~g}$ (1000 rpm in an Eppendorf $5810 \mathrm{R}$ ) at $4^{\circ} \mathrm{C}$ and the lysate was transferred to a $2 \mathrm{ml}$ Eppendorf tube. To remove the majority of unbroken cells and cell debris, the lysate was centrifuged at $1503 \mathrm{~g}$ (4000 rpm) for 2 minutes in an Eppendorf $5424 \mathrm{R}$ centrifuge at $4^{\circ} \mathrm{C}$. The supernatant was transferred to a fresh $2 \mathrm{ml}$ Eppendorf tube. Either the whole extract was sonicated in a $15 \mathrm{ml}$ bioruptor pico tube with $300 \mu$ l of sonication beads (Diagenode) added (Fig 5B-5D) or 2x $300 \mu$ l was sonicated in 2x $1.5 \mathrm{ml}$ bioruptor pico microtubes (Diagenode, Fig 6-9). When using the APLP protease inhibitors, before sonication $4.5 \mu$ l aprotinin, $0.15 \mu$ l leupeptin, $0.15 \mu \mathrm{l}$ pepstatin and $3 \mu \mathrm{l}$ PMSF was added to each tube containing $300 \mu \mathrm{l}$ chromatin. The samples were sonicated for 3 cycles (Fig 5 and 6A) or 4 cycles (Fig 6C-6F, 7 and 8) 30 seconds on / 30 seconds off or 10 cycles 15 seconds on / 30 seconds off (Fig 9). The chromatin was snap frozen in liquid nitrogen and stored at $-80^{\circ} \mathrm{C}$, except for a 20 $\mu \mathrm{l}$ aliquot that was taken to assess the extent of fragmentation of the DNA.

\section{Quality control after sonication}

The aliquot that was taken after sonication was diluted to $95 \mu \mathrm{l}$ by addition of 90 $\mu \mathrm{l}$ (zymolyase protocol and CE, full protocol) or $75 \mu \mathrm{l}$ (short bead beat protocol and WCL, full protocol) TE/SDS (10 mM Tris-HCl pH 8.0, $1 \mathrm{mM}$ EDTA pH 8.0, 1\% SDS) and decross-linked overnight at $65^{\circ} \mathrm{C}$. The next morning the decross-linked chromatin was treated with $5 \mu$ l of RNAse A/T1 (Thermo Scientific \#EN0551) for 30 $\min$ at $37^{\circ} \mathrm{C}$. Subsequently, proteins were digested by addition of $40 \mu \mathrm{l}$ proteinase $\mathrm{K}$ (Roche \#03115852001, dissolved to $10 \mu \mathrm{g} / \mu \mathrm{l}$ ) and incubation for 2 hours at $37^{\circ} \mathrm{C}$. The DNA was isolated using a Qiagen PCR purification cleanup kit (Qiagen \#28106). The standard cleanup protocol was used, except for the wash with PE buffer, which was done three times using $500 \mu \mathrm{l}$ PE buffer. The DNA was eluted in $40 \mu \mathrm{l}$ buffer EB. The degree of DNA fragmentation was assessed by running $1 \mu \mathrm{l}$ of each sample on a High Sensitivity DNA bioanalyzer chip. The fragmentation was considered good if the peak of the distribution of DNA fragment sizes was between 200-300 bp. If the peak of a specific sample was bigger than $300 \mathrm{bp}$, the chromatin was fragmented for a number of additional cycles to make the distribution similar to that of the other samples.

\section{Immunoprecipitation using agarose beads}

The immunoprecipitation with agarose beads was performed using anti-V5-agarose beads (Sigma-Aldrich \#A7345), which are agarose beads that are conjugated to a mono-clonal anti-V5 antibody. The calculated amount of beads for all IPs that were done at the same time ( $20 \mu \mathrm{l}$ per IP) was pre-washed together three times in FA lysis buffer (50 mM HEPES-KOH pH 7.5, 150 mM NaCl, 1 mM EDTA pH 8.0, 1\% Triton $\mathrm{X}-100,0.1 \%$ Na-deoxycholate, $0.1 \%$ SDS) by resuspending the beads in $1 \mathrm{ml}$ of FA lysis buffer and centrifuging for 1 minute at $845 \mathrm{~g}$ (3000 rpm) in an Eppendorf 5424 at RT. During the last wash, the beads were divided over $1.5 \mathrm{ml}$ Eppendorf tubes such that there was an equivalent of $20 \mu$ l beads per tube. Per IP, the beads were mixed with $250 \mu \mathrm{l}$ chromatin and incubated for 2 hours at RT. The beads were then pelleted 
by centrifugation at maximum speed $(21130 \mathrm{~g}, 15000 \mathrm{rpm})$ for a few seconds in an Eppendorf $5424 \mathrm{R}$ centrifuge and the supernatant was removed using a 23G needle and a syringe. The beads were subseqently washed twice in FA lysis buffer, twice with wash buffer 1 (FA lysis buffer containing $0.5 \mathrm{M} \mathrm{NaCl}$ ), and twice with wash buffer 2 (10 mM Tris pH 8.0, $0.25 \mathrm{mM} \mathrm{LiCl,} 1 \mathrm{mM}$ EDTA pH 8.0, 0.5\% Nonidet P-40 and $0.5 \%$ Na-deoxycholate) by centrifugation to maximum speed $(21130 \mathrm{~g}, 15000$ rpm) for a few seconds. After the last wash, all liquid was removed and the beads were resuspended in $100 \mu \mathrm{TE} / \mathrm{SDS}(10 \mathrm{mM}$ Tris- $\mathrm{HCl} \mathrm{pH}$ 8.0, $1 \mathrm{mM}$ EDTA pH 8.0, 1\% SDS). $2 \mu$ of RNAse A/T1 (Thermo Scientific \#EN0551) was added and the beads were incubated overnight at $65^{\circ} \mathrm{C}$ in a thermoshaker. The next morning, proteins were digested by addition of $40 \mu \mathrm{l}$ proteinase $\mathrm{K}$ (Roche, dissolved to $10 \mu \mathrm{g} / \mu \mathrm{l}$ ) and incubation for 2 hours at $37^{\circ} \mathrm{C}$. After protein digestion, the DNA was recovered using a Qiagen PCR purification cleanup kit (Qiagen \#28106). The standard cleanup protocol was used, except that the wash with PE buffer was done three times using $500 \mu \mathrm{l}$ PE buffer. The DNA was eluted in $40 \mu \mathrm{l}$ buffer EB.

\section{Immunoprecipitation using magnetic beads}

The anti-V5 antibody (Life Technologies \#R96025) was bound to the chromatin by incubating $150 \mu \mathrm{l}$ (Fig 4B), $200 \mu \mathrm{l}$ (Fig 2D, 2E, 3 and 5), $250 \mu \mathrm{l}$ (Fig 2A-2C and 4A) or $450 \mu \mathrm{l}$ (Fig 5B, 6-9) chromatin with $1 \mu \mathrm{l}$ (Fig 2D, 2E, 3-9) or $2 \mu \mathrm{l}$ (Fig 2A- 2C) of the anti-V5 antibody, either overnight (Fig 2A-2D and 4A), for 1 hour (Fig 2D), 2 hours (Fig 2D, 2E, 3-9) or 4 hours (Fig 2D) in a rotating wheel at $4^{\circ} \mathrm{C}$ (Fig 7C and 7C) or RT (Fig 2-9). For the samples with separate protease inhibitors added (Fig 8 and 9), in addition, also $15 \mu \mathrm{l}$ aprotinin (Sigma-Aldrich \#A6279), $0.5 \mu \mathrm{l}$ leupeptin (SigmaAldrich \#L2884, $1 \mathrm{mg} / \mathrm{ml}$ in MQ), $0.5 \mu \mathrm{l}$ pepstatin (Sigma-Aldrich \#P4265: $1 \mathrm{mg} / \mathrm{ml}$ in 100\% Methanol) and $5 \mu \mathrm{l}$ PMSF (Sigma-Aldrich: \#P7626, $200 \mathrm{mM}$ in isopropanol) were added right before the incubation.

During the incubation of chromatin with the antibody, the magnetic beads were prepared. For each IP, either $50 \mu \mathrm{l}$ (Fig 2A-2C, 7 and 8) or $25 \mu$ l (Fig 2D, 2E, 3-7 and 9) magnetic beads (Dynabeads protein G, Life Technologies \#10004D) was used. The preparation was done in either one of two ways. For the experiments shown in Fig 2A-2D and 4A the amount of beads was taken for the number of IPs +0.5 and transferred to a $1.5 \mathrm{ml}$ Eppendorf tube. The beads were separated on a magnetic stand (DynaMag-2, Life Technologies \#12321D) and the supernatant was removed. The beads were washed once in PBS-T (PBS pH 7.4, 0.02\% Tween-20) by pipetting up and down. The beads were then divided over $1.5 \mathrm{ml}$ Eppendorf tubes, separated using a magnetic stand and the supernatant was removed. When the beads were pre-incubated with BSA (Fig 2E, 3, 4B, 5-9) the beads were prepared as follows. 25 $\mu \mathrm{l}$ (Fig 2E, 3, 4B, 5-7 and 9) or $50 \mu \mathrm{l}$ (Fig 8) of magnetic beads per IP were transferred to a $1.5 \mathrm{ml}$ Eppendorf tube and separated on a magnetic stand. The supernatant was removed and the beads were resuspended in $500 \mu \mathrm{l}$ of PBS-T by gentle vortexing. After separating on the magnetic stand and removal of the supernatant, the beads were resuspended in $200 \mu \mathrm{l}$ PBS $+12.5 \mu \mathrm{l}$ of BSA $(10 \mathrm{mg} / \mathrm{ml}$ in TBS-T: $150 \mathrm{mM}$ $\mathrm{NaCl}, 10 \mathrm{mM}$ Tris pH 7.5, 0.05\% Tween-20, Fig 2E, 3, 4B, 5 and 6A), or $400 \mu \mathrm{PBS}$ $+25 \mu \mathrm{l}$ of BSA (Fig 6C-6F, 7-9) and incubated at $4^{\circ} \mathrm{C}$ until the chromatin + anti-V5 
antibody incubation was finished. 10 minutes before the end of the incubation of the chromatin with the antibody, the beads were centrifuged for 5 seconds to collect them in the bottom of the tube and subsequently separated on a magnetic stand. The supernatant was removed and the beads were washed once with $500 \mu \mathrm{l}$ PBS-T. After removing the supernatant, the chromatin + antibody was added to the beads. For the experiments shown in Fig 8 and 9, in addition, $5 \mu$ of PMSF was added. The beads, chromatin and antibody combination was resuspended and incubated at RT (Fig 2-9) or $4^{\circ} \mathrm{C}$ (Fig 7C and 7D) for 20 minutes (Fig 2-9) or 60 minutes (Fig 7C and 7D) in a rotating wheel. For the "pre incubation $\mathrm{Ab}+$ beads" samples shown in Fig 2C, the beads were incubated overnight with $2 \mu \mathrm{l}$ anti-V5 antibody in $200 \mu \mathrm{l}$ PBS-T at $4^{\circ} \mathrm{C}$ after washing. The next day, the supernatant was removed and the antibody conjugated beads were incubated with the chromatin in a rotating wheel for 20 minutes at RT.

After the incubation of the beads with the chromatin, the beads were washed by separating them on a magnetic stand and resuspended using either gentle pipetting (Fig 2A-2D, 4A) or gentle vortexing (Fig 2E, 3, 4B, 5-9). The beads were washed with any of the washes: twice with PBS and once with PBS-T (Fig 2-9, standard wash), once with PBS (Fig 7B: low wash), twice with FA-lysis buffer, twice with wash buffer 1 (FA lysis buffer containing $0.5 \mathrm{M} \mathrm{NaCl})$ and twice with wash buffer $2(10 \mathrm{mM}$ Tris pH 8.0, $0.25 \mathrm{mM} \mathrm{LiCl}, 1 \mathrm{mM}$ EDTA pH 8.0, 0.5\% Nonidet P-40 and 0.5\% Nadeoxycholate) (Fig 7B: high wash 1) or twice with PBS, twice with wash buffer 1 and twice with PBS-T (Fig 7B: high wash 2). The beads were then resuspended in $100 \mu \mathrm{l}$ PBS-T and transferred to a fresh $1.5 \mathrm{ml}$ DNA LoBind Tube (Eppendorf \#0030108051). After removal of the PBS-T, the cells were resuspended in $95 \mu \mathrm{l}$ (Fig 2A-2C) or $98 \mu \mathrm{l}$ (Fig 2D, 2E, 3-9) TE/SDS (10 mM Tris-HCl pH 8.0, 1 mM EDTA pH 8.0, 1\% SDS) and decross-linked at $65^{\circ} \mathrm{C}$ overnight in a thermoshaker. As an input control, $5 \mu \mathrm{l}$ (Fig 2-5), $10 \mu \mathrm{l}$ (Fig 7), $11.25 \mu \mathrm{l}$ (Fig 5B) or $20 \mu \mathrm{l}$ (Fig 6-9) of the chromatin that was not incubated with the antibody was also decross-linked overnight after dilution to 95 $\mu \mathrm{l}$ with TE/SDS at $65^{\circ} \mathrm{C}$ in a thermoshaker. For the glycine eluted samples (Fig 2B), the beads were first resuspended in $20 \mu \mathrm{l} 50 \mathrm{mM}$ glycine $\mathrm{pH} 2.8$ and incubated for 2 minutes. The beads were separated and the supernatant was mixed with $75 \mu \mathrm{TE} /$ SDS, and subsequently the beads were resuspended in $95 \mu \mathrm{l}$ of TE/SDS. Both were decross-linked overnight at $65^{\circ} \mathrm{C}$.

The next morning, RNA was first degraded by addition of $2 \mu$ (IP: Fig 2D, 2E, 3-9) or $5 \mu \mathrm{l}$ (IP: Fig 2A-2C and input: Fig 2-9) RNAse A/T1 (Thermo Scientific \#EN0551) and incubation the beads at $37^{\circ} \mathrm{C}$ for 30 minutes. Subsequently, proteins were digested by addition of $40 \mu \mathrm{l}$ proteinase $\mathrm{K}$ (Roche $\# 03115852001$, dissolved to $10 \mu \mathrm{g} / \mu \mathrm{l}$ ) and incubation for 2 hours at $37^{\circ} \mathrm{C}$. After protein digestion, the DNA was recovered using a Qiagen PCR purification cleanup kit (Qiagen \#28106). The standard cleanup protocol was used, except that the wash with PE buffer was done three times using $500 \mu \mathrm{l}$ PE buffer. The DNA was eluted in $30 \mu \mathrm{l}$ (Fig 2, 4A) or $40 \mu \mathrm{l}$ (Fig 3, 4B, 5-9) buffer EB. 


\section{Western blot}

The integrity of the proteins of interest was assessed using Western blots at different steps during the protocol. $5 \mu \mathrm{l}$ (Fig 9), $20 \mu \mathrm{l}$ (Fig 4, 5) or $40 \mu \mathrm{l}$ (Fig 8) aliquots were taken at the indicated steps, snap frozen and stored at $-80^{\circ} \mathrm{C}$. The pre-lysis samples and the control samples were lysed by resuspending the cells in $200 \mu \mathrm{l}$ of $0.1 \mathrm{M}$ $\mathrm{NaOH}$ and incubating them for 5 minutes at RT. After centrifugation at $3381 \mathrm{~g}$ (6000 rpm) in an 5424 Eppendorf centrifuge at RT, the supernatant was discarded and the cell pellet was resuspended in 2x sample buffer (2\% SDS, $80 \mathrm{mM}$ Tris $\mathrm{pH} 6.8,10$ $\%$ glycerol, $572 \mathrm{mM} \beta$-mercaptoethanol, 0.016\% bromophenol blue). These samples were heated for 5 minutes (non-cross-linked controls) or 30 minutes (cross-linked, pre-lysis samples) at $95^{\circ} \mathrm{C}$ before loading $10 \mu \mathrm{l}$ on a protein gel.

The post-lysis and post-sonication samples were diluted with 5x sample buffer (5\% SDS, $200 \mathrm{mM}$ Tris pH 6.8, 25\% glycerol, $1.43 \mathrm{M} \beta$-mercaptoethanol, $0.032 \%$ bromophenol blue). $10 \mu \mathrm{l}$ of sample was mixed with $2.5 \mu \mathrm{l}$ of $5 \mathrm{x}$ sample buffer (Fig 9), $20 \mu \mathrm{l}$ was mixed with $20 \mu \mathrm{l}$ of 5x sample buffer (Fig 4 and 5) or $40 \mu \mathrm{l}$ was mixed with $10 \mu \mathrm{l} 5 \mathrm{x}$ sample buffer (Fig 8). The cross-linked samples were decrosslinked for 30 minutes at $95^{\circ} \mathrm{C}$ before loading $10 \mu \mathrm{l}$ on the gel. Either regular (Fig 4 and 5) or stain-free (Fig 8 and 9, Bio-Rad \#1610182) $10 \%$ acrylamide gels were used. $5 \mu \mathrm{l}$ of a broad range protein marker (New England Biolabs, \#P7708) was run alongside the samples on each gel to estimate the molecular weight. After the samples migrated through the gel, the proteins were transferred to a nitrocellulose membrane (Amersham Protran nitrocellulose membrane \#10600001) overnight in transfer buffer containing 10\% methanol. After transfer, the membrane was first washed with TBS-T (150 mM NaCl, $10 \mathrm{mM}$ Tris $\mathrm{pH} 7.5,0.05 \%$ Tween-20) and then blocked in TBS-T containing 2\% protifar (Nutricia \#56317) either for 1 hour at RT or overnight at $4^{\circ} \mathrm{C}$. After two washes with TBS-T, the membrane was incubated with an anti-V5 antibody (Life Technologies \#R96025) and incubated for 1.5 hours at RT. The blot was washed 4 times with TBS-T and subsequently incubated with a goat anti-mouse-HRP antibody (Bio-Rad \#1706516) for 30 minutes. The blot was again washed 3 times with TBS-T and the last wash was performed using TBS. The blot was then imaged after incubation with ECL (PerkinElmer, Western Lightning Plus \#NEL105001EA) on a chemidoc touch imaging system (Bio-Rad).

The quantification of the percentage of intact protein was performed as follows. An image of each blot was taken without any satured pixels. The image was exported for analysis as a TIFF image using the Image Lab software (Bio-Rad) and analyzed in the ImageJ software (Schneider et al, 2012). Using the rectangle tool, a rectangle was drawn around each intact protein band and the sum of the pixels was recorded. The same sized rectangle was used for each intact protein band on the same gel. The amount of degraded signal was determined by drawing a rectangle that encompassed all the visible lower bands and recording the sum of the pixels in this rectangle. Since each protein had a different number of degradation bands, a differently sized rectangle was used for the degraded bands of each protein. The signal of the intact and degraded bands was background corrected by subtracting the sum of the pixels of an identically shaped rectangle that was drawn somewhere 
on the blot where there were no protein bands present. The percent intact protein was then calculated by dividing the signal from the intact protein band by the sum of the signals from the intact and the degraded protein bands. This is represented by the following formula:

$$
\% \text { degraded }=\frac{\text { intact protein }- \text { background }}{\text { intact protein }- \text { background }+ \text { degraded protein }- \text { background }}
$$

\section{qPCR}

DNA binding levels were quantified using qPCR. qPCRs were performed in 384well plates (Bio-Rad white hard-shell plate \#hsp3805) in a final volume of $10 \mu$ l. For each reaction, $5 \mu \mathrm{l}$ IQ SYBR Green super mix (Bio-Rad \#1708886), $2.8 \mu \mathrm{MQ}, 0.2 \mu \mathrm{l}$ primer mix (containing $10 \mu \mathrm{M}$ of both the forward and the reverse primer), 0.014 $\mu \mathrm{l}$ precision blue (Bio-Rad \#1725555) and $2 \mu \mathrm{l}$ template were mixed. First, a master mix was made of MQ and IQ SYBR Green for the total number of reactions + 20\%. Subsequently, a separate master mix was made for each primer pair for the number of reactions for each primer pair $+\sim 10 \%$. After addition of the primers, precision blue was added to this master mix using a 1:700 dilution, to aid pipetting in the 384well plate. An electronic pipette was used to accurately pipette $8 \mu \mathrm{l}$ of this mix to the wells of the 384-well plate. Next, $2 \mu \mathrm{l}$ template or MQ was added to each well using an 8-channel multichannel pipette.

On each plate, a 5 times, 10-fold serially diluted standard curve was taken along for each primer pair, together with at least 2 no template controls (MQ). All IP measurements were performed either undiluted or 2 times diluted and were performed in technical quadruplicate. The inputs were diluted 10, 25, 50 or 100 times, depending on the experiment, and were either done in technical triplicate or quadruplicate. qPCRs were performed using a CFX384 Touch Real-Time PCR Detection system (Bio-Rad) with a 2-step PCR protocol. First, the DNA was denatured for 30 seconds at $95^{\circ} \mathrm{C}$, followed by 40 cycles of 15 seconds denaturing at $95^{\circ} \mathrm{C}$ and 30 seconds of amplification at $60^{\circ} \mathrm{C}$. The amplification was followed by a melting curve that started with 1 minute denaturing at $95^{\circ} \mathrm{C}$ and then ramped up from $70^{\circ} \mathrm{C}$ with half a degree every 5 seconds to $95^{\circ} \mathrm{C}$. All primers were designed to have a melting temperature above $60^{\circ} \mathrm{C}$ in the IQ SYBR green super mix.

In all figures the binding is shown as a percentage of input. This was calculated by first calculating the starting quantities (SQ) for each IP and input for each primer pair using the CFX Meastro (Bio-Rad) software. These values were then exported and the average value of the replicates was calculated. If there was a single measurement that was too high or low due to a pipetting error, this measurement was removed before calculating the average. The starting quantities were first corrected for the dilution and subsequently for volume, since for the inputs only a small fraction of the material was used compared to the IP ( 5\%). After this correction, the signal from the IP was divided by the signal from the input for each biological replicate, which yields the percent of input. The average of the biological replicates is shown 
in the figures. The variation was calculated by using the distance from the mean when duplicates were used or by using the standard deviation when more than two replicates were used.

\section{Author contributions}

Conceptualization, W.d.J., P.K and F.H.; Investigation, W.d.J. and M.B.; Writing Original Draft, W.d.J. and F.H; Writing - Review \& Editing, W.d.J., M.B., P.K and F.H.; Funding Acquisition, P.K and F.H.; Supervision, P.K and F.H.

\section{Acknowledgements}

We thank the members of the Holstege and Kemmeren groups for their support and discussions. We thank Tineke Lenstra and Marc Timmers for advice regarding the protocol. We are grateful to Jeff DeMartino for critical reading of the manuscript. This work was supported by the Netherlands Organisation for Scientific Research (NWO) grant 86411010 and by the European Research Council (ERC) grant 671174 DynaMech.

\section{References}

Acevedo LG, Leonardo Iniguez A, Holster HL, Zhang X, Green R, Farnham PJ (2007) Genomescale ChIP-chip analysis using 10,000 human cells. BioTechniques 43: 791-797

Adli M, Zhu J, Bernstein BE (2010) Genome-wide chromatin maps derived from limited numbers of hematopoietic progenitors. Nat Methods 7: 615-618

Arrigoni L, Al-Hasani H, Ramírez F, Panzeri I, Ryan DP, Santacruz D, Kress N, Pospisilik JA, Bönisch U, Manke T (2018) RELACS nuclei barcoding enables high-throughput ChIP-seq. Commun Biol 1: 214

Arrigoni L, Richter AS, Betancourt E, Bruder K, Diehl S, Manke T, Bönisch U (2016) Standardizing chromatin research: a simple and universal method for ChIP-seq. Nucleic Acids Res 44: e67

Baranello L, Kouzine F, Sanford S, Levens D (2015) ChIP bias as a function of cross-linking time. Chromosome Res 24: 175-181

Brind'Amour J, Liu S, Hudson M, Chen C, Karimi MM, Lorincz MC (2015) An ultra-low-input native ChIP-seq protocol for genome-wide profiling of rare cell populations. Nat Commun 6: 6033

Buchman AR, Kornberg RD (1990) A yeast ARS-binding protein activates transcription synergistically in combination with other weak activating factors. Mol Cell Biol 10: 887-897

Bulyk ML, Huang X, Choo Y, Church GM (2001) Exploring the DNA-binding specificities of zinc fingers with DNA microarrays. Proc Natl Acad Sci U S A 98: 7158-7163

Cai M, Davis RW (1990) Yeast centromere binding protein CBF1, of the helix-loop-helix protein family, is required for chromosome stability and methionine prototrophy. Cell 61: 437-446

Chasman DI, Lue NF, Buchman AR, LaPointe JW, Lorch Y, Kornberg RD (1990) A yeast protein that influences the chromatin structure of UASG and functions as a powerful auxiliary gene activator. Genes Dev 4: 503-514 
Cremazy FGE, Manders EMM, Bastiaens PIH, Kramer G, Hager GL, van Munster EB, Verschure PJ, Gadella TJ, van Driel R (2005) Imaging in situ protein-DNA interactions in the cell nucleus using FRET-FLIM. Exp Cell Res 309: 390-396

Dahl JA, Collas P (2007) Q2ChIP, a Quick and Quantitative Chromatin Immunoprecipitation Assay, Unravels Epigenetic Dynamics of Developmentally Regulated Genes in Human Carcinoma Cells. Stem Cells 25: 1037-1046

Dedon PC, Soults JA, David Allis C, Gorovsky MA (1991) A simplified formaldehyde fixation and immunoprecipitation technique for studying protein-DNA interactions. Anal Biochem 197: 83-90

Dey B, Thukral S, Krishnan S, Chakrobarty M, Gupta S, Manghani C, Rani V (2012) DNAprotein interactions: methods for detection and analysis. Mol Cell Biochem 365: 279-299

Ellington AD, Szostak JW (1990) In vitro selection of RNA molecules that bind specific ligands. Nature 346: 818

Field J, Nikawa J, Broek D, MacDonald B, Rodgers L, Wilson IA, Lerner RA, Wigler M (1988) Purification of a RAS-responsive adenylyl cyclase complex from Saccharomyces cerevisiae by use of an epitope addition method. Mol Cell Biol 8: 2159-2165

Galas DJ, Schmitz A (1978) DNAse footprinting: a simple method for the detection of proteinDNA binding specificity. Nucleic Acids Res 5: 3157-3170

Ganapathi M, Palumbo MJ, Ansari SA, He Q, Tsui K, Nislow C, Morse RH (2011) Extensive role of the general regulatory factors, Abf1 and Rap1, in determining genome-wide chromatin structure in budding yeast. Nucleic Acids Res 39: 2032-2044

Garner MM, Revzin A (1981) A gel electrophoresis method for quantifying the binding of proteins to specific DNA regions: application to components of the Escherichia coli lactose operon regulatory system. Nucleic Acids Res 9: 3047-3060

Ghaemmaghami S, Huh W-K, Bower K, Howson RW, Belle A, Dephoure N, O'Shea EK, Weissman JS (2003) Global analysis of protein expression in yeast. Nature 425: 737-741

Gilmour DS, Lis JT (1984) Detecting protein-DNA interactions in vivo: distribution of RNA polymerase on specific bacterial genes. Proc Natl Acad Sci U S A 81: 4275-4279

Goren A, Ozsolak F, Shoresh N, Ku M, Adli M, Hart C, Gymrek M, Zuk O, Regev A, Milos PM, Bernstein BE (2010) Chromatin profiling by directly sequencing small quantities of immunoprecipitated DNA. Nat Methods 7: 47-49

Gutin J, Sadeh R, Bodenheimer N, Joseph-Strauss D, Klein-Brill A, Alajem A, Ram O, Friedman N (2018) Fine-Resolution Mapping of TF Binding and Chromatin Interactions. Cell Rep 22: 2797-2807

Haber JE (2012) Mating-Type Genes and MAT Switching in Saccharomyces cerevisiae. Genetics 191: 33-64

Hartley PD, Madhani HD (2009) Mechanisms that specify promoter nucleosome location and identity. Cell 137: 445-458

Haruki H, Nishikawa J, Laemmli UK (2008) The anchor-away technique: rapid, conditional establishment of yeast mutant phenotypes. Mol Cell 31: 925-932

He Q, Johnston J, Zeitlinger J (2015) ChIP-nexus enables improved detection of in vivo transcription factor binding footprints. Nat Biotechnol 33: 395-401

Hebbes TR, Thorne AW, Crane-Robinson C (1988) A direct link between core histone acetylation and transcriptionally active chromatin. EMBO J 7: 1395-1402

Hoffman EA, Frey BL, Smith LM, Auble DT (2015) Formaldehyde Crosslinking: A Tool for the Study of Chromatin Complexes. J Biol Chem 290: 26404-26411

Hopp TP, Prickett KS, Price VL, Libby RT, March CJ, Cerretti DP, Urdal DL, Conlon PJ (1988) A Short Polypeptide Marker Sequence Useful for Recombinant Protein Identification and Purification. Bio/Technology 6: 1204

Irlbacher H, Franke J, Manke T, Vingron M, Ehrenhofer-Murray AE (2005) Control of replication initiation and heterochromatin formation in Saccharomyces cerevisiae by a 
regulator of meiotic gene expression. Genes Dev 19: 1811-1822

Jackson V (1978) Studies on histone organization in the nucleosome using formaldehyde as a reversible cross-linking agent. Cell 15: 945-954

de Jonge WJ, O'Duibhir E, Lijnzaad P, van Leenen D, Groot Koerkamp MJ, Kemmeren P, Holstege FC (2017) Molecular mechanisms that distinguish TFIID housekeeping from regulatable SAGA promoters. EMBO J 36: 274-290

Ju QD, Morrow BE, Warner JR (1990) REB1, a yeast DNA-binding protein with many targets, is essential for growth and bears some resemblance to the oncogene myb. Mol Cell Biol 10: 5226-5234

Kasinathan S, Orsi GA, Zentner GE, Ahmad K, Henikoff S (2014) High-resolution mapping of transcription factor binding sites on native chromatin. Nat Methods 11: 203-209

Kawashima Y, Kodera Y, Singh A, Matsumoto M, Matsumoto H (2014) Efficient extraction of proteins from formalin-fixed paraffin-embedded tissues requires higher concentration of tris(hydroxymethyl)aminomethane. Clin Proteomics 11: 4

Kemmeren P, Sameith K, van de Pasch LAL, Benschop JJ, Lenstra TL, Margaritis T, O’Duibhir E, Apweiler E, van Wageningen S, Ko CW, van Heesch S, Kashani MM, AmpatziadisMichailidis G, Brok MO, Brabers NACH, Miles AJ, Bouwmeester D, van Hooff SR, van Bakel H, Sluiters E, et al (2014) Large-scale genetic perturbations reveal regulatory networks and an abundance of gene-specific repressors. Cell 157: 740-752

Kent NA, Eibert SM, Mellor J (2004) Cbf1p is required for chromatin remodeling at promoterproximal CACGTG motifs in yeast. J Biol Chem 279: 27116-27123

Kuo MH, Allis CD (1999) In vivo cross-linking and immunoprecipitation for studying dynamic Protein:DNA associations in a chromatin environment. Methods San Diego Calif 19: 425-433

Kuo MH, Grayhack E (1994) A library of yeast genomic MCM1 binding sites contains genes involved in cell cycle control, cell wall and membrane structure, and metabolism. Mol Cell Biol 14: 348-359

Lara-Astiaso D, Weiner A, Lorenzo-Vivas E, Zaretsky I, Jaitin DA, David E, Keren-Shaul H, Mildner A, Winter D, Jung S, Friedman N, Amit I (2014) Immunogenetics. Chromatin state dynamics during blood formation. Science 345: 943-949

Lushnikov AY, Potaman VN, Oussatcheva EA, Sinden RR, Lyubchenko YL (2006) DNA strand arrangement within the SfiI-DNA complex: atomic force microscopy analysis. Biochemistry 45: 152-158

MacIsaac KD, Wang T, Gordon DB, Gifford DK, Stormo GD, Fraenkel E (2006) An improved map of conserved regulatory sites for Saccharomyces cerevisiae. BMC Bioinformatics 7: 113

Maerkl SJ, Quake SR (2007) A systems approach to measuring the binding energy landscapes of transcription factors. Science 315: 233-237

McIsaac RS, Petti AA, Bussemaker HJ, Botstein D (2012) Perturbation-based analysis and modeling of combinatorial regulation in the yeast sulfur assimilation pathway. Mol Biol Cell 23: 2993-3007

Messenguy F, Dubois E (2003) Role of MADS box proteins and their cofactors in combinatorial control of gene expression and cell development. Gene 316: 1-21

Miyake T, Reese J, Loch CM, Auble DT, Li R (2004) Genome-wide analysis of ARS (autonomously replicating sequence) binding factor 1 (Abf1p)-mediated transcriptional regulation in Saccharomyces cerevisiae. J Biol Chem 279: 34865-34872

Nick H, Gilbert W (1985) Detection in vivo of protein-DNA interactions within the lac operon of Escherichia coli. Nature 313: 795-798

O'Neill LP, Turner BM (2003) Immunoprecipitation of native chromatin: NChIP. Methods San Diego Calif 31: 76-82

O'Neill LP, VerMilyea MD, Turner BM (2006) Epigenetic characterization of the early embryo with a chromatin immunoprecipitation protocol applicable to small cell populations. Nat 
Genet 38: 835-841

Park D, Lee Y, Bhupindersingh G, Iyer VR (2013) Widespread misinterpretable ChIP-seq bias in yeast. PloS One 8: e83506

Passmore S, Maine GT, Elble R, Christ C, Tye BK (1988) Saccharomyces cerevisiae protein involved in plasmid maintenance is necessary for mating of MAT alpha cells. J Mol Biol 204: 593-606

Pchelintsev NA, Adams PD, Nelson DM (2016) Critical Parameters for Efficient Sonication and Improved Chromatin Immunoprecipitation of High Molecular Weight Proteins. PloS One 11: e0148023

Poorey K, Viswanathan R, Carver MN, Karpova TS, Cirimotich SM, McNally JG, Bekiranov S, Auble DT (2013) Measuring Chromatin Interaction Dynamics on the Second Time Scale at Single-Copy Genes. Science 342: 369-372

Reed SH, Akiyama M, Stillman B, Friedberg EC (1999) Yeast autonomously replicating sequence binding factor is involved in nucleotide excision repair. Genes Dev 13: 3052-3058

Rhee HS, Pugh BF (2011) Comprehensive genome-wide protein-DNA interactions detected at single-nucleotide resolution. Cell 147: 1408-1419

Rhode PR, Elsasser S, Campbell JL (1992) Role of multifunctional autonomously replicating sequence binding factor 1 in the initiation of DNA replication and transcriptional control in Saccharomyces cerevisiae. Mol Cell Biol 12: 1064-1077

Rhode PR, Sweder KS, Oegema KF, Campbell JL (1989) The gene encoding ARS-binding factor I is essential for the viability of yeast. Genes Dev 3: 1926-1939

Schmid M, Durussel T, Laemmli UK (2004) ChIC and ChEC; genomic mapping of chromatin proteins. Mol Cell 16: 147-157

Schmidl C, Rendeiro AF, Sheffield NC, Bock C (2015) ChIPmentation: fast, robust, low-input ChIP-seq for histones and transcription factors. Nat Methods 12: 963-965

Schneider CA, Rasband WS, Eliceiri KW (2012) NIH Image to ImageJ: 25 years of image analysis. Nat Methods 9: 671-675

Siebenlist U, Gilbert W (1980) Contacts between Escherichia coli RNA polymerase and an early promoter of phage T7. Proc Natl Acad Sci U S A 77: 122-126

Siebenlist U, Simpson RB, Gilbert W (1980) E. coli RNA polymerase interacts homologously with two different promoters. Cell 20: 269-281

Skene PJ, Henikoff S (2015) A simple method for generating high-resolution maps of genomewide protein binding. eLife 4: e09225

Skene PJ, Henikoff S (2017) An efficient targeted nuclease strategy for high-resolution mapping of DNA binding sites. eLife 6: e21856

Smith GP (1985) Filamentous fusion phage: novel expression vectors that display cloned antigens on the virion surface. Science 228: 1315-1317

Solomon MJ, Larsen PL, Varshavsky A (1988) Mapping proteinDNA interactions in vivo with formaldehyde: Evidence that histone $\mathrm{H} 4$ is retained on a highly transcribed gene. Cell 53: 937-947

Southern JA, Young DF, Heaney F, Baumgärtner WK, Randall RE (1991) Identification of an epitope on the $\mathrm{P}$ and $\mathrm{V}$ proteins of simian virus 5 that distinguishes between two isolates with different biological characteristics. J Gen Virol 72: 1551-1557

van Steensel B, Henikoff S (2000) Identification of in vivo DNA targets of chromatin proteins using tethered dam methyltransferase. Nat Biotechnol 18: 424-428

Sutherland BW, Toews J, Kast J (2008) Utility of formaldehyde cross-linking and mass spectrometry in the study of protein-protein interactions. J Mass Spectrom JMS 43: 699-715

Teytelman L, Thurtle DM, Rine J, van Oudenaarden A (2013) Highly expressed loci are vulnerable to misleading ChIP localization of multiple unrelated proteins. Proc Natl Acad Sci U S A 110: 18602-18607

Tuerk C, Gold L (1990) Systematic evolution of ligands by exponential enrichment: RNA 
ligands to bacteriophage T4 DNA polymerase. Science 249: 505-510

Wallerman O, Nord H, Bysani M, Borghini L, Wadelius C (2015) lobChIP: from cells to sequencing ready ChIP libraries in a single day. Epigenetics Chromatin 8: 25

Wang MM, Reed RR (1993) Molecular cloning of the olfactory neuronal transcription factor Olf-1 by genetic selection in yeast. Nature 364: 121-126

Xie J, Pierce M, Gailus-Durner V, Wagner M, Winter E, Vershon AK (1999) Sum1 and Hst1 repress middle sporulation-specific gene expression during mitosis in Saccharomyces cerevisiae. EMBO J 18: 6448-6454

Yin H, Wang MD, Svoboda K, Landick R, Block SM, Gelles J (1995) Transcription against an applied force. Science 270: 1653-1657

Zaidi H, Hoffman EA, Shetty SJ, Bekiranov S, Auble DT (2017) Second-generation method for analysis of chromatin binding with formaldehyde-cross-linking kinetics. J Biol Chem 292: 19338-19355

Zaret KS, Mango SE (2016) Pioneer transcription factors, chromatin dynamics, and cell fate control. Curr Opin Genet Dev 37: 76-81

Zentner GE, Kasinathan S, Xin B, Rohs R, Henikoff S (2015) ChEC-seq kinetics discriminates transcription factor binding sites by DNA sequence and shape in vivo. Nat Commun 6: 8733 


\section{Methods per figure}

\section{Figure 2}

Figure $2 A$

Cbf1-aa Stb4-V5 cells used for Fig 2A-2C were prepared in a big batch, by growing several $250 \mathrm{ml}$ cultures. Cultures were cross-linked with $1 \%$ formaldehyde by adding $6.8 \mathrm{ml}$ of $37 \%$ formaldehyde and incubating them for 5 minutes. Subsequently, 12.8 $\mathrm{ml} 2.5 \mathrm{M}$ glycine was added to a final concentration of $125 \mathrm{mM}$ and incubated for 5 minutes. Cells were harvested as described in the general methods section.

Six cell pellets of Cbf1-aa strains were lysed using the zymolyase protocol, by treating them with zymolyase solution $(10 \mathrm{mg} / \mathrm{ml}$ in buffer Z) for 10 minutes. After two careful washes with buffer Z, each cell pellet was resuspended in $540 \mu$ FA lysis buffer. All samples were pooled, mixed and split over $12 \times 1.5 \mathrm{ml}$ sonication tubes, by putting $300 \mu \mathrm{l}$ in each tube. Half of the samples were sonicated 30 seconds on / 30 seconds off for 3 cycles and the other half for 4 cycles in a bioruptor pico. All samples that were sheared for the same number of cycles were pooled and $250 \mu \mathrm{l}$ of chromatin was used for each IP.

Eight samples (4 that were sonicated for 3 cycles and 4 that were sonicated for 4 cycles) were used for the IP with magnetic beads while 4 samples ( 2 that were sonicated for 3 cycles and 2 that were sonicated for 4 cycles) were used for the IP using agarose beads. The IPs with magnetic and agarose beads were performed as described in the general methods section with some modifications. $50 \mu \mathrm{l}$ beads and $2 \mu \mathrm{l}$ anti-V5 antibody were used for the IPs with magnetic beads, while $20 \mu \mathrm{l}$ of antiV5-agarose beads were used. The agarose beads were prepared by washing 3 times in FA lysis buffer prior to use, while the magnetic beads were washed with $500 \mu \mathrm{l}$ PBS-T. After incubation with the chromatin, the agarose beads were washed using FA lysis buffer, wash buffer 1 and wash buffer 2 as described in the general methods section. Several replicates of the magnetic IPs were performed with different washes. Two IPs with material that was sonicated for 3 cycles were washed with the standard washes of $2 x$ PBS and 2x PBS-T. Two IPs with material that was sonicated for 4 cycles were washed by doing 3 washes using PBS. The other four IPs (two that were sonicated for 3 cycles and two that were sonicated for 4 cycles) were washed first $2 x$ with PBS and then $2 x$ using wash buffer 2 . The DNA was recovered by eluting overnight in $\mathrm{TE} / \mathrm{SDS}$ at $65^{\circ} \mathrm{C}$. Although the washes were different between the samples, the average of all the agarose beads IPs (four replicates) and the average of all the magnetic beads IPs (eight replicates) are shown in Fig 2A.

Figure $2 B$ and $2 C$

The results shown in Fig $\mathbf{2 B}$ and $\mathbf{2 C}$ were performed in the same experiment on the same day. The results were split over 2 figures for clarity. The same samples are shown in Fig 2B: TE/SDS and Fig 2C: 20 min beads inc.

Cbf1-aa Stb4-V5 cells used for Fig 2A-2C were prepared in a big batch, by growing 
several $250 \mathrm{ml}$ cultures. The cultures were cross-linked with $1 \%$ formaldehyde by adding $6.8 \mathrm{ml}$ of $37 \%$ formaldehyde and incubating them for 5 minutes. Subsequently, $12.8 \mathrm{ml} 2.5 \mathrm{M}$ glycine was added to a final concentration of $125 \mathrm{mM}$ and incubated for 5 minutes. The cells were harvested as described in the general methods section.

Five pellets were lysed using the zymolyase protocol, by incubating them in zymolyase solution ( $10 \mathrm{mg} / \mathrm{ml}$ in buffer Z) for 10 minutes. After two careful washes with buffer $Z$, each cell pellet was resuspended in $550 \mu \mathrm{l}$ FA lysis buffer. All samples were pooled, mixed and split over $10 \times 1.5 \mathrm{ml}$ sonication tubes, by putting $300 \mu \mathrm{l}$ in each tube. The samples were sonicated for 3 cycles, 30 seconds on / 30 seconds off. All samples were pooled and $250 \mu \mathrm{l}$ of chromatin was used for each IP.

The IPs with magnetic beads were performed as described in the general methods section, with some modifications. $50 \mu \mathrm{l}$ magnetic beads and $2 \mu \mathrm{l}$ anti-V5 antibody were used for the IPs. Prior to the incubation, the beads were washed with $500 \mu \mathrm{l}$ PBS-T. For the IPs shown in Fig $\mathbf{2 B}$ and $\mathbf{2 C}$, the antibody was incubated with the chromatin overnight at $4^{\circ} \mathrm{C}$, except for the samples "pre inc $\mathrm{Ab}+$ beads", where the beads were incubated with the antibody overnight at $4^{\circ} \mathrm{C}$ in $200 \mu \mathrm{l}$ PBS-T. For Fig 2B, the chromatin + antibody was bound to the beads by incubating them with the beads at RT for 20 minutes. For Fig 2C, this incubation was done for either 20 minutes or 60 minutes. In addition, the antibody conjugated beads were incubated with the chromatin at RT for 20 minutes as well. The beads were washed with $2 x$ PBS and $2 x$ PBS-T. The DNA was eluted either by incubating the beads overnight at $65^{\circ} \mathrm{C}$ in 98 $\mu \mathrm{TE} / \mathrm{SDS}$ or by incubating the beads in $20 \mu \mathrm{l} 0 \mathrm{mM}$ glycine $\mathrm{pH} 2.8$ for 2 minutes. The glycine eluted beads were separated from the supernatant and the beads were resuspended in $98 \mu \mathrm{TE} / \mathrm{SDS}$ and a second elution was done overnight at $65^{\circ} \mathrm{C}$. The $20 \mu \mathrm{l}$ supernatant was mixed with $75 \mu \mathrm{lTE} / \mathrm{SDS}$ and incubated overnight at $65^{\circ} \mathrm{C}$ as well. All IPs were performed in duplicate.

\section{Figure 2D}

Cbf1-aa cells used in Fig 2D were prepared in a big batch, by growing several $250 \mathrm{ml}$ cultures. The cultures were cross-linked with $1 \%$ formaldehyde by adding $6.8 \mathrm{ml}$ of $37 \%$ formaldehyde to the cultures and incubating them for 5 minutes. Subsequently, $12.8 \mathrm{ml} 2.5 \mathrm{M}$ glycine was added to a final concentration of $125 \mathrm{mM}$ and incubated for 5 minutes. The cells were harvested as described in the general methods section.

Six Cbf1-aa pellets were lysed using the zymolyase protocol, by incubating them in zymolyase solution $(10 \mathrm{mg} / \mathrm{ml}$ in buffer Z) for 10 minutes. After two careful washes with buffer $Z$, each cell pellet was resuspended in $550 \mu \mathrm{l} F$ A lysis buffer. All samples were pooled, mixed and split over $12 \times 1.5 \mathrm{ml}$ sonication tubes, by putting $300 \mu \mathrm{l}$ in each tube. The samples were sonicated for 3 cycles, 30 seconds on / 30 seconds off. 3 tubes were pooled and $200 \mu \mathrm{l}$ was used for the IPs of the overnight samples. The next day, the rest of the tubes were pooled and $200 \mu \mathrm{l}$ of this pool was used for each IP where the chromatin and antibody were incubated for 1-4 hours.

The IPs with magnetic beads were performed as described in the general methods 
section with some modifications. $25 \mu \mathrm{l}$ magnetic beads and $1 \mu \mathrm{l}$ anti-V5 antibody were used for the IPs. The chromatin was incubated with the anti-V5 antibody for 1 hour, 2 hours, 4 hours or overnight. The incubations were staggered such that all IPs were ready at the same time, and all washes were performed in parallel. The standard wash of $2 x$ PBS and $2 x$ PBS-T was used for all samples. The DNA was recovered by eluting overnight in TE/SDS at $65^{\circ} \mathrm{C}$. The IPs were performed in triplicate.

\section{Figure 2E}

Cbf1-aa cells used in Fig 2E were grown as $100 \mathrm{ml}$ cultures. The cells were crosslinked by adding $2.7 \mathrm{ml}$ of $37 \%$ formaldehyde to a final concentration of $1 \%$ and incubating them for 5 minutes. Subsequently, $5.1 \mathrm{ml}$ of $2.5 \mathrm{M}$ glycine was added to a final concentration of $125 \mathrm{mM}$ and the cells were incubated for 5 minutes. The cells were harvested as described in the general methods section.

The Cbf1-aa pellets were lysed using the zymolyase protocol, by incubating them in zymolyase solution ( $10 \mathrm{mg} / \mathrm{ml}$ in buffer Z) for 10 minutes. After two careful washes with buffer $Z$, each cell pellet was resuspended in $550 \mu \mathrm{l} F A$ lysis buffer. All samples were pooled, mixed and split over $12 \times 1.5 \mathrm{ml}$ sonication tubes, by putting $300 \mu \mathrm{l}$ in each tube. The samples were sonicated for 3 cycles, 30 seconds on / 30 seconds off and the sheared chromatin was pooled per cell pellet and $200 \mu \mathrm{l}$ of chromatin was used for each IP.

The IPs with magnetic beads were performed as described in the general methods section with some modifications. $25 \mu \mathrm{l}$ magnetic beads and $1 \mu \mathrm{l}$ anti-V5 antibody were used for the IPs. The chromatin was incubated with the anti-V5 antibody for 2 hours. During the incubation of the chromatin with the antibody, the beads were prepared. $25 \mu \mathrm{l}$ beads per IP was washed in individual $1.5 \mathrm{ml}$ Eppendorf tubes using $500 \mu \mathrm{l}$ PBS-T. For the samples without BSA, right before the addition of the chromatin and antibody, the beads were washed once in $500 \mu \mathrm{l}$ PBS-T. For the sample with BSA, the beads were resuspended in $200 \mu \mathrm{l}$ PBS, $12.5 \mu \mathrm{l} \mathrm{BSA}(10 \mathrm{mg} / \mathrm{ml}$ in TBS-T) was added and the beads were incubated at $4{ }^{\circ} \mathrm{C}$ while the chromatin was incubating with the antibody. Just before this incubation was finished, the beads were washed again with $500 \mu \mathrm{l}$ PBS-T. The standard wash of $2 x$ PBS and 2x PBS-T was used for all samples. The DNA was recovered by eluting overnight in TE/SDS at $65^{\circ} \mathrm{C}$. The IPs were performed in triplicate.

\section{Figure 3}

\section{Figure $3 A$ and $3 B$}

The results shown in Fig 3A and 3B were performed in the same experiment on the same day. The results were split over two figures for clarity. The same samples are shown for 5 min $125 \mathrm{mM}$ glycine in both Fig 3A and 3B.

Cbf1-aa Cha4-V5 cells used for Fig 3A and 3B were grown as $100 \mathrm{ml}$ cultures. The cells were cross-linked by adding $2.7 \mathrm{ml}$ of $37 \%$ formaldehyde to a final concentration of $1 \%$ and incubating them for 1 minute. Subsequently, $5.1 \mathrm{ml}$ of $2.5 \mathrm{M}$ glycine was 
added to a final concentration of $125 \mathrm{mM}$ and the cells were incubated for 1,5 or 10 minutes. In addition, some samples were incubated with $10.7 \mathrm{ml} 2.5 \mathrm{M}$ glycine, at final concentration of $250 \mathrm{mM}$ instead of $125 \mathrm{mM}$, and incubated for 5 minutes. The cells were harvested as described in the general methods section.

The Cbf1-aa Cha4-V5 pellets were lysed using the zymolyase protocol, by incubating them in zymolyase solution $(10 \mathrm{mg} / \mathrm{ml}$ in buffer $\mathrm{Z})$ for 10 minutes. After two careful washes with buffer $Z$, each cell pellet was resuspended in $550 \mu \mathrm{l} \mathrm{FA} \mathrm{lysis} \mathrm{buffer.} \mathrm{The}$ samples were split over $2 \times 1.5 \mathrm{ml}$ sonication tubes, by putting $300 \mu \mathrm{l}$ in each tube. All samples were sonicated for 3 cycles, 30 seconds on / 30 seconds off and the sheared chromatin was pooled per cell pellet. $200 \mu \mathrm{l}$ of chromatin was used for each IP.

The IPs with magnetic beads were performed as described in the general methods section. $25 \mu \mathrm{l}$ magnetic beads and $1 \mu \mathrm{l}$ anti-V5 antibody were used for the IPs. The chromatin was incubated with the anti-V5 antibody for 2 hours. During the incubation of the chromatin with the antibody, the beads were prepared. $25 \mu \mathrm{l}$ beads per IP was washed in individual $1.5 \mathrm{ml}$ Eppendorf tubes using $500 \mu \mathrm{l}$ PBS-T. The beads were resuspended in $200 \mu \mathrm{l}$ PBS, $12.5 \mu \mathrm{l}$ BSA $(10 \mathrm{mg} / \mathrm{ml}$ in TBS-T) was added and the beads were incubated at $4^{\circ} \mathrm{C}$ while the chromatin was incubating with the antibody. Just before this incubation was finished, the beads were washed again with $500 \mu \mathrm{l}$ PBS-T. The standard wash of 2x PBS and 2x PBS-T was used for all samples. The DNA was recovered by eluting overnight in TE/SDS at $65^{\circ} \mathrm{C}$. The IPs were performed in triplicate.

\section{Figure $3 C$ and $3 D$}

Cbf1-aa cells used for Fig 3A and 3B were grown as $100 \mathrm{ml}$ cultures. The cells were cross-linked by adding $2.7 \mathrm{ml}$ of $37 \%$ formaldehyde to a final concentration of $1 \%$ and incubating them for 1 minute $(1 \mathrm{~min}$ non-quenched, glycine quenched and Tris quenched samples) or 6 minutes (6 min non-quenched sample). Samples that were not quenched were immediately transferred to $50 \mathrm{ml}$ tubes and centrifuged as described in the general methods sections. To the glycine quenched samples, $5.1 \mathrm{ml}$ of $2.5 \mathrm{M}$ glycine was added to a final concentration of $125 \mathrm{mM}$ and the cells were incubated for 5 minutes. The Tris quenched samples were incubated with 20.5 $\mathrm{ml} 4.5 \mathrm{M}$ Tris $\mathrm{pH} 8.0$ at a final concentration of $750 \mathrm{mM}$ for 1, 5 or 10 minutes. All samples were harvested as described in the general methods section.

The Cbf1-aa pellets were lysed using the zymolyase protocol, by incubating them in zymolyase solution $(10 \mathrm{mg} / \mathrm{ml}$ in buffer Z) for 10 minutes. After two careful washes with buffer Z, each cell pellet was resuspended in $550 \mu \mathrm{l} \mathrm{FA} \mathrm{lysis} \mathrm{buffer.} \mathrm{All} \mathrm{samples}$ were split over $2 \times 1.5 \mathrm{ml}$ sonication tubes, by putting $300 \mu \mathrm{l}$ in each tube. The samples were sonicated for 3 cycles, 30 seconds on / 30 seconds off and the sheared chromatin was pooled per cell. $200 \mu \mathrm{l}$ of chromatin was used for each IP.

The IPs with magnetic beads were performed as described in the general methods section. $25 \mu \mathrm{l}$ magnetic beads and $1 \mu \mathrm{l}$ anti-V5 antibody were used for the IPs. The chromatin was incubated with the anti-V5 antibody for 2 hours. During the 
incubation of the chromatin with the antibody, the beads were prepared. $25 \mu$ l beads per IP was washed in individual $1.5 \mathrm{ml}$ Eppendorf tubes using $500 \mu \mathrm{l}$ PBS-T. The beads were resuspended in $200 \mu \mathrm{l}$ PBS, $12.5 \mu \mathrm{l}$ BSA $(10 \mathrm{mg} / \mathrm{ml}$ in TBS-T) was added and the beads were incubated at $4^{\circ} \mathrm{C}$ while the chromatin was incubating with the antibody. Just before this incubation was finished, the beads were washed again with $500 \mu \mathrm{l}$ PBS-T. The standard wash of 2x PBS and 2x PBS-T was used for all samples. The DNA was recovered by eluting overnight in TE/SDS at $65^{\circ} \mathrm{C}$. The IPs were performed in triplicate.

\section{Figure 4}

\section{Figure $4 A$}

Cbf1-aa cells used in Fig 4A were grown in the same big batch as those used for Fig 2D, by growing several $250 \mathrm{ml}$ cultures. The cultures were cross-linked with $1 \%$ formaldehyde by adding $6.8 \mathrm{ml}$ of $37 \%$ formaldehyde and incubating them for 5 minutes. Subsequently, $12.8 \mathrm{ml} 2.5 \mathrm{M}$ glycine was added to a final concentration of $125 \mathrm{mM}$ and incubated for 5 minutes. The cells were harvested as described in the general methods section.

The Cbf1-aa pellets were lysed using the zymolyase protocol, by incubating them in zymolyase solution ( $10 \mathrm{mg} / \mathrm{ml}$ in buffer Z) for 10 or 25 minutes. After two careful washes with buffer $Z$, each cell pellet was resuspended in $550 \mu \mathrm{l} \mathrm{FA} \mathrm{lysis} \mathrm{buffer.} \mathrm{The}$ samples were split over $2 \times 1.5 \mathrm{ml}$ sonication tubes, by putting $300 \mu \mathrm{l}$ in each tube. All samples were sonicated for 3 cycles, 30 seconds on / 30 seconds off and the sheared chromatin was pooled per cell pellet. $200 \mu \mathrm{l}$ of chromatin was used for each IP.

The IPs with magnetic beads were performed as described in the general methods section. $25 \mu \mathrm{l}$ magnetic beads and $1 \mu \mathrm{l}$ anti-V5 antibody were used for the IPs. The chromatin was incubated with the anti-V5 antibody for 2 hours. The beads were washed once in $500 \mu \mathrm{l}$ PBS-T, the chromatin and antibody was added to the beads and incubated at RT for 20 minutes. The beads were washed with the standard wash of 2x PBS and 2x PBS-T and DNA was recovered by eluting overnight in TE/SDS at $65^{\circ} \mathrm{C}$. The IPs were performed in triplicate.

\section{Figure $4 B$ and $4 C$}

Cbf1-aa cells used in Fig $4 \mathrm{~B}$ and $4 \mathrm{C}$ were grown in the same big batch as those used for Fig 2D and 4A, by growing several $250 \mathrm{ml}$ cultures. The cultures were cross-linked with $1 \%$ formaldehyde by adding $6.8 \mathrm{ml}$ of $37 \%$ formaldehyde and incubating them for 5 minutes. Subsequently, $12.8 \mathrm{ml} 2.5 \mathrm{M}$ glycine was added to a final concentration of $125 \mathrm{mM}$ and incubated for 5 minutes. The cells were harvested as described in the general methods section.

Four Cbf1-aa pellets, which are equivalent to $100 \mathrm{ml}$ cell cultures OD 0.8, plus some left over Cbf1-aa material was resuspended in $720 \mu \mathrm{l}$ buffer $\mathrm{Z}$ and divided over 6 tubes. A $20 \mu \mathrm{l}$ aliquot was taken as a pre-lysis control for Western blotting. The cells were lysed using the zymolyase protocol, by incubating them in zymolyase 
solution (10 $\mathrm{mg} / \mathrm{ml}$ in buffer $\mathrm{Z}$ ) for 10 minutes. Two samples were incubated without protease inhibitors and 4 samples were incubated with protease inhibitors (one tablet of EDTA-free cOmplete protease inhibitor cocktail (Roche \#11873580001) per $10 \mathrm{ml}$ of zymolyase solution). The samples that were lysed without protease inhibitors and two of the samples that were lysed with protease inhibitors were washed twice with buffer $Z$ without protease inhibitors. The other two samples that were lysed in the presence of protease inhibitors were washed with buffer $Z$ containing one tablet cOmplete protease inhibitor cocktail (Roche \#11836145001) per $10 \mathrm{ml}$ buffer $\mathrm{Z}$. After the washes, each cell pellet was resuspended in $550 \mu \mathrm{FA}$ lysis buffer and a $20 \mu \mathrm{l}$ aliquot was taken for Western blotting. The samples were split over $2 \times 1.5 \mathrm{ml}$ sonication tubes, by putting $300 \mu \mathrm{l}$ in each tube, and were sonicated for 3 cycles, 30 seconds on / 30 seconds off. The sheared chromatin was pooled per cell pellet and for each sample a $20 \mu \mathrm{l}$ aliquot was taken for Western blotting both before and after the centrifugation step. Because there were multiple aliquots taken for Western blotting, only $150 \mu \mathrm{l}$ of chromatin was left to use in each IP.

The IPs with magnetic beads were performed as described in the general methods section. $25 \mu \mathrm{l}$ magnetic beads and $1 \mu \mathrm{l}$ anti-V5 antibody were used for the IPs. The chromatin was incubated with the anti-V5 antibody for 2 hours. $25 \mu \mathrm{l}$ beads per IP was washed in individual $1.5 \mathrm{ml}$ Eppendorf tubes using $500 \mu \mathrm{l}$ PBS-T. The beads were resuspended in $200 \mu \mathrm{l}$ PBS, $12.5 \mu \mathrm{lBSA}(10 \mathrm{mg} / \mathrm{ml}$ in TBS-T) was added and the beads were incubated at $4^{\circ} \mathrm{C}$ while the chromatin was incubating with the antibody. Just before this incubation was finished, the beads were washed again with $500 \mu \mathrm{l}$ PBS-T. The chromatin and antibody were added to the beads, and incubated at RT for 20 minutes. The beads were washed with the standard wash of $2 x$ PBS and $2 x$ PBS-T and DNA was recovered by eluting overnight in TE/SDS at $65^{\circ} \mathrm{C}$. The IPs were performed in duplicate. The results shown in Fig $4 \mathrm{~B}$ are the average of two IPs (without inhibitors) or of four IPs (with inhibitors). The average signal of the samples with inhibitors during the zymolyase treatment is shown, regardless of whether or not they were washed with buffer $\mathrm{Z}$ containing protease inhibitors.

Samples for Western blotting were prepared by mixing $20 \mu \mathrm{l}$ of the sample with $20 \mu \mathrm{l}$ of $5 \mathrm{X}$ sample buffer (5\% SDS, $200 \mathrm{mM}$ Tris $\mathrm{pH} 6.8,25 \%$ glycerol, $1.43 \mathrm{M}$ $\beta$-mercaptoethanol, $0.032 \%$ bromophenol blue). The pre-lysis samples were prepared as described in the general methods section, by lysing the cells using $\mathrm{NaOH}$. All but the control samples were heated to $95^{\circ} \mathrm{C}$ for 30 minutes to reverse cross-links. The samples $(10 \mu \mathrm{l})$ were separated on a $10 \%$ polyacrylamide gel. As a control, a noncross-linked crude lysate of a WT-aa and a Cbf1-aa strain were taken along, which were heated to $95^{\circ} \mathrm{C}$ for 5 minutes prior to loading. A chromatin extract of a Cbf1aa Cha4-V5 strain prepared with the full bead beating protocol was also included. After the proteins had migrated through the gel, the proteins were transferred to a nitrocellulose membrane. The membrane was stained with an anti-V5 antibody (Life Technologies \#R96025) and binding was visualized using a goat-anti-mouseHRP conjugated antibody (Bio-Rad \#1706516) and ECL solution, as described in the general methods section. 


\section{Figure 5}

\section{Figure 5A}

Cbf1-aa Cha4-V5 cells used in Fig 5A were grown as $100 \mathrm{ml}$ cultures. The cells were cross-linked by adding $2.7 \mathrm{ml}$ of $37 \%$ formaldehyde to a final concentration of $1 \%$ and incubating them for 5 minutes. Subsequently, $20.5 \mathrm{ml}$ of $4.5 \mathrm{M}$ Tris pH 8.0 was added to a final concentration of $750 \mathrm{mM}$ and the cells were incubated for 1 minute. The cells were harvested as described in the general methods section

Cells were lysed either using zymolyase or the full bead beating protocol. When using zymolyase to lyse the cells, the Cbf1-aa Cha4-V5 cell pellets were incubated in zymolyase solution $(10 \mathrm{mg} / \mathrm{ml}$ in buffer Z) for 10 minutes. After two careful washes with buffer $Z$, each cell pellet was resuspended in $550 \mu \mathrm{FA}$ lysis buffer. The samples were split over $2 \times 1.5 \mathrm{ml}$ sonication tubes, by putting $300 \mu \mathrm{l}$ in each tube. All samples were sonicated for 3 cycles, 30 seconds on / 30 seconds off. Sheared chromatin was pooled per cell pellet. The samples that did not have appropriate fragment lengths were sheared for an additional cycle. $200 \mu \mathrm{l}$ of chromatin was used for each IP. The cells that were lysed with the bead beating protocol were bead beated for $7 \times 3$ minutes in a genie disruptor. The cell debris was pelleted and the lysate centrifuged at $18407 \mathrm{~g}$ for 15 minutes to pellet the chromatin. The chromatin was then washed with FA lysis buffer and resuspended in $600 \mu \mathrm{l}$ FA lysis buffer. The chromatin was subsequently fragmented by splitting each sample over 2 x $1.5 \mathrm{ml}$ sonication tubes and sonicating 30 seconds on / 30 seconds off. The sheared chromatin was pooled per cell pellet. $200 \mu \mathrm{l}$ chromatin was used for each IP.

IPs with magnetic beads were performed as described in the general methods section. $25 \mu \mathrm{l}$ magnetic beads and $1 \mu \mathrm{l}$ anti-V5 antibody were used for the IPs. The chromatin was incubated with the anti-V5 antibody for 2 hours. $25 \mu$ l beads per IP was washed in individual $1.5 \mathrm{ml}$ Eppendorf tubes using $500 \mu \mathrm{l}$ PBS-T. The beads were resuspended in $200 \mu \mathrm{l}$ PBS, $12.5 \mu \mathrm{lBSA}(10 \mathrm{mg} / \mathrm{ml}$ in TBS-T) was added and the beads were incubated at $4^{\circ} \mathrm{C}$ while the chromatin was incubating with the antibody. Just before this incubation was finished, the beads were washed again with $500 \mu \mathrm{l}$ PBS-T. The chromatin and antibody were added to the beads and incubated at RT for 20 minutes. The beads were washed with the standard wash of $2 x$ PBS and $2 x$ PBS-T and DNA was recovered by eluting overnight in TE/SDS at $65^{\circ} \mathrm{C}$. The IPs were performed in triplicate.

Figure 5B-5D

Cbf1-aa Cha4-V5 cells used in Fig 5B-5D were grown in a big batch, by growing several $250 \mathrm{ml}$ cultures. The cultures were cross-linked with $1 \%$ formaldehyde by adding $7.0 \mathrm{ml}$ of $37 \%$ formaldehyde and incubating them for 5 minutes. Subsequently, $32.1 \mathrm{ml} 4.5 \mathrm{M}$ Tris was added to a final concentration of $500 \mathrm{mM}$ and incubated for 1 minute. The cells were harvested as described in the general methods section.

The Cbf1-aa Cha4-V5 cells were lysed either using the zymolyase protocol, the full bead beating protocol or the short bead beating protocol. 4 Cbf1-aa Cha4-V5 cell 
pellets were resuspended in $1 \mathrm{ml}$ of Buffer $\mathrm{Z}$ and a $20 \mu \mathrm{l}$ aliquot was taken as a prelysis control for Western blotting. The cells were then lysed using the zymolyase protocol, by incubating them in zymolyase solution $(10 \mathrm{mg} / \mathrm{ml}$ zymolyase in buffer $\mathrm{Z}$ containing one tablet of EDTA-free cOmplete protease inhibitor cocktail (Roche $\# 11873580001$ ) per $10 \mathrm{ml}$ ) for 10 minutes. The samples were subsequently washed with buffer $\mathrm{Z}$ containing one tablet Roche cOmplete protease inhibitor cocktail (Roche \#11873580001) per $10 \mathrm{ml}$ buffer Z. After the washes, each cell pellet was resuspended in $550 \mu \mathrm{l} \mathrm{FA}$ lysis buffer and a $20 \mu \mathrm{l}$ aliquot was taken for Western blotting (pre-sonication). The samples were split over 2 x $1.5 \mathrm{ml}$ sonication tubes, by putting $300 \mu \mathrm{l}$ in each tube and were sonicated for 3 cycles, 30 seconds on / 30 seconds off. All sheared chromatin was pooled per cell pellet and for each sample a $20 \mu \mathrm{l}$ aliquot was taken for Western blotting.

The other samples were lysed using either the full or the short bead beating protocol. 8 Cbf1-aa Cha4-V5 pellets were resuspended in $1 \mathrm{ml}$ of FA lysis buffer and a $20 \mu \mathrm{l}$ aliquot was taken for Western blotting. The tubes were filled with FA lysis buffer and the cells were subsequently bead beated for $7 \times 3$ minutes in a genie disruptor. The cell debris was pelleted and for the 4 samples that were processed with the full bead beat protocol, the lysate was centrifuged at $18407 \mathrm{~g}$ for 15 minutes to pellet the chromatin, which contains the CE. The rest of the lysate (WCL) was kept on ice. The CE was then washed once with FA lysis buffer and resuspended in $600 \mu \mathrm{l}$ FA lysis buffer. A $20 \mu \mathrm{l}$ sample was taken for Western blotting. The CE was subsequently fragmented by splitting each sample over $2 \times 1.5 \mathrm{ml}$ sonication tubes and sonicating for 3 cycles, 30 seconds on / 30 seconds off. The sheared chromatin was pooled per cell pellet. Before sonicating the WCL and the short bead beat chromatin, a $20 \mu \mathrm{l}$ sample was taken for Western Blotting, and then the samples were sheared in $15 \mathrm{ml}$ sonication tubes containing $300 \mu \mathrm{l}$ sonication beads (Diagenode), also for 3 cycles 30 seconds on / 30 seconds off. A $20 \mu \mathrm{l}$ sample was taken for Western blotting from 2 samples of all 3 protocols after the sonication. $200 \mu \mathrm{l}$ of the CE was used for IPs, while $450 \mu \mathrm{l}$ of the WCL and the short bead beat chromatin was used.

The IPs with magnetic beads were performed as described in the general methods section. $25 \mu \mathrm{l}$ magnetic beads and $1 \mu \mathrm{l}$ anti-V5 antibody were used for the IPs. The chromatin was incubated with the anti-V5 antibody for 2 hours. $25 \mu$ l beads per IP was washed in individual $1.5 \mathrm{ml}$ Eppendorf tubes using $500 \mu \mathrm{l}$ PBS-T. The beads were resuspended in $200 \mu \mathrm{l}$ PBS, $12.5 \mu \mathrm{lBSA}(10 \mathrm{mg} / \mathrm{ml}$ in TBS-T) was added and the beads were incubated at $4^{\circ} \mathrm{C}$ while the chromatin was incubating with the antibody. Just before this incubation was finished, the beads were washed again with $500 \mu \mathrm{l}$ PBS-T. The chromatin and antibody were added to the beads and incubated at RT for 20 minutes. The beads were washed with the standard wash of $2 x$ PBS and $2 x$ PBS-T and DNA was recovered by eluting overnight in TE/SDS at $65^{\circ} \mathrm{C}$. The IPs were performed either in duplicate (WCL) or quadruplicate (CE and short bead beat protocol).

To calculate the relative amount of input DNA, the starting quantities (SQs) of the input samples from Fig $5 B$ were taken and the average SQ of the inputs of the CE 
was set to $100 \%$. The values of all input samples were scaled accordingly.

The samples for Western blotting were prepared by mixing $20 \mu \mathrm{l}$ of the sample with $20 \mu \mathrm{l}$ of 5X sample buffer (5\% SDS, $200 \mathrm{mM}$ Tris $\mathrm{pH}$ 6.8, 25\% glycerol, 1.43 M $\beta$-mercaptoethanol, $0.032 \%$ bromophenol blue). The pre-lysis samples were prepared as described in the general methods section, by lysing the cells using $\mathrm{NaOH}$. All samples were heated to $95^{\circ} \mathrm{C}$ for 30 minutes to reverse cross-links. The samples $(10 \mu \mathrm{l})$ were separated on a $10 \%$ polyacrylamide gel. As a control, a noncross-linked crude lysate of a Cbf1-aa strain, heated to $95^{\circ} \mathrm{C}$ for 5 minutes prior to loading, was taken along on both gels. After the proteins had migrated through the gel, the proteins were transferred to a nitrocellulose membrane. The membrane was stained with an anti-V5 antibody (Life Technologies \#R96025) and binding was visualized using a goat-anti-mouse-HRP conjugated antibody (Bio-Rad \#1706516) and ECL solution, as described in the general methods section.

\section{Figure 6}

\section{Figure $6 \mathrm{~A}$}

Cbf1-aa Cha4-V5 cells used in Fig 6A were grown as $100 \mathrm{ml}$ cultures. At OD = 0.52, 1 hour before the cells reached OD $=0.8,367.4 \mu \mathrm{l} 2 \mathrm{mM}$ rapamycin $(60 \mathrm{~min}$ depleted samples, final concentration $7.5 \mu \mathrm{M}$ ) or $367.4 \mu \mathrm{l}$ or DMSO (non-depleted samples) was added. The cells that were cross-linked (depleted and non-depleted) were incubated with $2.7 \mathrm{ml}$ of $37 \%$ formaldehyde to a final concentration of $1 \%$ for 5 minutes. Subsequently, $20.5 \mathrm{ml}$ of $4.5 \mathrm{M}$ Tris $\mathrm{pH} 8.0$ was added to a final concentration of $750 \mathrm{mM}$ and the cells were incubated for 1 minute. The non-cross-linked samples were either incubated for 6 minutes with $20.5 \mathrm{ml}$ of $4.5 \mathrm{M}$ Tris $\mathrm{pH} 8.0$ at a final concentration of $750 \mathrm{mM}$ or first incubated with $20.5 \mathrm{ml}$ of $4.5 \mathrm{M}$ Tris $\mathrm{pH} 8.0$ for 1 minute and then $2.7 \mathrm{ml}$ of $37 \%$ formaldehyde was added to a final concentration of $1 \%$ for 5 minutes. The cells were harvested as described in the general methods section

The Cbf1-aa cells were bead beated for $7 \times 3$ minutes in a genie disruptor. The cell debris was pelleted and the supernatant was used for sonication. The chromatin was fragmented by splitting each sample over 2 x $1.5 \mathrm{ml}$ sonication tubes and sonicating 30 seconds on / 30 seconds off. The sheared chromatin was pooled per cell pellet. 450 $\mu \mathrm{l}$ of chromatin was used for each IP.

The IPs with magnetic beads were performed as described in the general methods section. $25 \mu \mathrm{l}$ magnetic beads and $1 \mu \mathrm{l}$ anti-V5 antibody were used for the IPs. The chromatin was incubated with the anti-V5 antibody for 2 hours. $25 \mu$ l beads per IP was washed in individual $1.5 \mathrm{ml}$ Eppendorf tubes using $500 \mu \mathrm{l}$ PBS-T. The beads were resuspended in $200 \mu \mathrm{l}$ PBS, $12.5 \mu \mathrm{lBSA}(10 \mathrm{mg} / \mathrm{ml}$ in TBS-T) was added and the beads were incubated at $4^{\circ} \mathrm{C}$ while the chromatin was incubating with the antibody. Just before this incubation was finished, the beads were washed again with $500 \mu \mathrm{l}$ PBS-T. The chromatin and antibody were added to the beads and incubated at RT for 20 minutes. The beads were washed with the standard wash of $2 x$ PBS and $2 x$ 
PBS-T and DNA was recovered by eluting overnight in TE/SDS at $65^{\circ} \mathrm{C}$. The IPs were performed in triplicate, and the average of the samples where only Tris was added and the samples where Tris was added before the formaldehyde was used as the signal of the non-cross-linked control (6 replicates in total).

\section{Figure 6B}

Cbf1-aa cells were grown in $100 \mathrm{ml}$ cultures. 60 minutes (at $\mathrm{OD}=0.52$ ) and 15 minutes (at $\mathrm{OD}=0.72$ ) before the cells reached $\mathrm{OD}=0.8,367.4 \mu \mathrm{l} 2 \mathrm{mM}$ rapamycin was added to the different cultures (final concentration $7.5 \mu \mathrm{M}$ ). As a non-depleted control, a culture was taken along where $367.4 \mu \mathrm{DMSO}$ was added at OD $=0.52$. When the cultures were ready, a $1 \mathrm{ml}$ aliquot was taken which was fixed using $100 \%$ methanol as is described in the general methods section. $1.5 \mu \mathrm{l}$ was mixed with 1.5 $\mu \mathrm{l} \%$ agarose on a slide to image the cells.

\section{Figure $6 \mathrm{C}$ and $6 \mathrm{D}$}

Abf1-aa (Fig 6C) and Reb1-aa (Fig 6D) cells were grown as $100 \mathrm{ml}$ cultures. The cells were cross-linked by adding $2.7 \mathrm{ml}$ of $37 \%$ formaldehyde to a final concentration of $1 \%$ and incubating them for 5 minutes. Subsequently, $20.5 \mathrm{ml}$ of $4.5 \mathrm{M}$ Tris $\mathrm{pH}$ 8.0 was added to a final concentration of $750 \mathrm{mM}$ and the cells were incubated for 1 minute. There was no formaldehyde added to the non-cross-linked samples, but these samples were still incubated with Tris. The cells were harvested as described in the general methods section.

The Abf1-aa and Reb1-aa cells were bead beated for 7x 3 minutes in a genie disruptor. The cell debris was pelleted and the supernatant was used for sonication. The chromatin was fragmented by splitting each sample over $2 \times 1.5 \mathrm{ml}$ sonication tubes and sonicating for 4 cycles 30 seconds on / 30 seconds off. The sheared chromatin was pooled per cell pellet. $450 \mu \mathrm{l}$ of chromatin was used for each IP.

The IPs with magnetic beads were performed as described in the general methods section. $25 \mu \mathrm{l}$ magnetic beads and $1 \mu \mathrm{l}$ anti-V5 antibody were used for the IPs. The chromatin was incubated with the anti-V5 antibody for 2 hours. $25 \mu$ l beads per IP was washed in individual $1.5 \mathrm{ml}$ Eppendorf tubes using $500 \mu \mathrm{l}$ PBS-T. The beads were resuspended in $400 \mu \mathrm{l}$ PBS, $25 \mu \mathrm{lBSA}(10 \mathrm{mg} / \mathrm{ml}$ in TBS-T) was added and the beads were incubated at $4^{\circ} \mathrm{C}$ while the chromatin was incubating with the antibody. Just before this incubation was finished, the beads were washed again with $500 \mu \mathrm{l}$ PBS-T. The chromatin and antibody were added to the beads and incubated at RT for 20 minutes. The Abf1-aa samples were washed with the standard wash of $2 x$ PBS and 2x PBS-T, while the Reb1-aa were washed twice with wash buffer 1 (FA lysis buffer containing $500 \mathrm{mM} \mathrm{NaCl}$ ) before the standard washes. DNA was recovered by eluting overnight in TE/SDS at $65^{\circ} \mathrm{C}$. The IPs were performed in triplicate

\section{Figure 6 E and $6 F$}

The Abf1-aa (Fig 6E) and Reb1-aa (Fig 6F) cells were grown first as $200 \mathrm{ml}$ cultures. $750 \mu \mathrm{l} 2 \mathrm{mM}$ rapamycin was added to a final concentration of $7.5 \mu \mathrm{M}$ at OD=0.56 to deplete the proteins from the nucleus. To the non-depleted samples $750 \mu \mathrm{l}$ 
DMSO was added. $100 \mathrm{ml}$ per culture was cross-linked by adding $2.7 \mathrm{ml}$ of $37 \%$ formaldehyde to a final concentration of $1 \%$ and incubating them for 5 minutes. Subsequently, $20.5 \mathrm{ml}$ of $4.5 \mathrm{M}$ Tris $\mathrm{pH} 8.0$ was added to a final concentration of 750 $\mathrm{mM}$ and the cells were incubated for 1 minute. The cells were harvested as described in the general methods section.

The Abf1-aa and Reb1-aa cells were bead beated for 7x 3 minutes in a genie disruptor. The cell debris was pelleted and the supernatant was used for sonication. The chromatin was fragmented by splitting each sample over $2 \times 1.5 \mathrm{ml}$ sonication tubes and sonicating for 4 cycles 30 seconds on / 30 seconds off. The sheared chromatin was pooled per cell pellet. $450 \mu \mathrm{l}$ of chromatin was used for each IP.

The IPs with magnetic beads were performed as described in the general methods section. $25 \mu \mathrm{l}$ magnetic beads and $1 \mu \mathrm{l}$ anti-V5 antibody were used for the IPs. The chromatin was incubated with the anti-V5 antibody for 2 hours. $25 \mu \mathrm{l}$ beads per IP was washed in individual $1.5 \mathrm{ml}$ Eppendorf tubes using $500 \mu \mathrm{l}$ PBS-T. The beads were resuspended in $400 \mu \mathrm{l}$ PBS, $25 \mu \mathrm{l}$ BSA $(10 \mathrm{mg} / \mathrm{ml}$ in TBS-T) was added and the beads were incubated at $4^{\circ} \mathrm{C}$ while the chromatin was incubating with the antibody. Just before this incubation was finished, the beads were washed again with $500 \mu \mathrm{l}$ PBS-T. The chromatin and antibody were added to the beads and incubated at RT for 20 minutes. The beads were washed with the standard wash of $2 x$ PBS and $2 x$ PBS-T and DNA was recovered by eluting overnight in TE/SDS at $65^{\circ} \mathrm{C}$. The IPs were performed in triplicate

\section{Figure 7}

The results shown in Fig 7B, 7C and 7D were performed in the same experiments on the same days. The results were split over two figures for clarity. The same samples are shown in Fig 7B: Standard wash and Fig 7C and 7D: RT 20 min.

Sum1-aa cells used in Fig 7 were prepared in a big batch, by growing several $250 \mathrm{ml}$ cultures. The cultures were cross-linked with $1 \%$ formaldehyde by adding $7.0 \mathrm{ml}$ of $37 \%$ formaldehyde and incubating them for 5 minutes. Subsequently, $32.1 \mathrm{ml} 4.5 \mathrm{M}$ Tris was added to a final concentration of $500 \mathrm{mM}$ and incubated for 1 minute. The cells were harvested as described in the general methods section.

The Sum1-aa cells were bead beated for 7x 3 minutes in a genie disruptor. The cell debris was pelleted and the supernatant was used for sonication. The chromatin was fragmented by splitting each sample over $2 \times 1.5 \mathrm{ml}$ sonication tubes and sonicating for 4 cycles 30 seconds on / 30 seconds off. The sheared chromatin was pooled per cell pellet. $450 \mu \mathrm{l}$ of chromatin was used for each IP.

On each day that IPs were performed, several chromatin extracts were pooled and split again to use for the IPs (the results shown in Fig 7A were obtained on a different day than the results shown in Fig 7B-7D). $25 \mu$ ( Fig 7A) or $50 \mu$ (Fig 7B-7D) magnetic beads and $1 \mu \mathrm{l}$ anti-V5 antibody were used for the IPs. The chromatin was incubated 
with the anti-V5 antibody for 2 hours. $25 \mu \mathrm{l}$ beads per IP was washed in individual $1.5 \mathrm{ml}$ Eppendorf tubes using $500 \mu \mathrm{l}$ PBS-T. The beads were resuspended in $400 \mu \mathrm{l}$ PBS, $25 \mu \mathrm{BSA}\left(10 \mathrm{mg} / \mathrm{ml}\right.$ in TBS-T) was added and the beads were incubated at $4^{\circ} \mathrm{C}$ while the chromatin was incubating with the antibody. Just before this incubation was finished, the beads were washed again with $500 \mu \mathrm{l}$ PBS-T. The chromatin and antibody were added to the beads and incubated at RT for 20 minutes or at $4^{\circ} \mathrm{C}$ for 20 or 60 minutes (Fig 7D and 7D). The beads were washed with the standard wash of 2x PBS and 2x PBS-T, 1x with PBS and 1x with PBS-T (Fig 7B: low wash), with 2x FA lysis buffer, 2x wash buffer 1 (FA lysis buffer with $500 \mathrm{mM} \mathrm{NaCl}$ ), 2x wash buffer 2 (10 mM Tris pH 8.0, $0.25 \mathrm{mM} \mathrm{LiCl,} 1 \mathrm{mM}$ EDTA pH 8.0, 0.5\% Nonidet P-40 and $0.5 \%$ Na-deoxycholate) and $1 x$ PBS-T or with $2 x$ PBS, $2 x$ wash buffer 1 and $3 x$ PBS-T . DNA was recovered by eluting overnight in TE/SDS at $65^{\circ} \mathrm{C}$. For the samples that were eluted a second time (Fig 7A), the next morning the beads were separated from the supernatant and the beads were again incubated with $98 \mu \mathrm{l}$ of TE/SDS for 2 hours at $65^{\circ} \mathrm{C}$ before proteinase $\mathrm{K}$ treatment. The IPs were performed in duplicate (Fig 7A, 7B: High wash 1, and 7C: $4^{\circ} \mathrm{C} 20 \mathrm{~min}$ and $4^{\circ} \mathrm{C} 60 \mathrm{~min}$ and 7D: $4^{\circ} \mathrm{C} 20 \mathrm{~min}$ and $4^{\circ} \mathrm{C} 60 \mathrm{~min}$ ), triplicate (Fig 7B: standard wash and 7C: RT $20 \mathrm{~min}$ ) or quadruplicate (Fig 7B: Low wash and High wash2).

\section{Figure 8}

\section{Figure $8 A$ and $8 B$}

Abf1-aa strains used in Fig 8A and 8B were grown in a big batch, by growing several $250 \mathrm{ml}$ cultures. The cultures were cross-linked with $1 \%$ formaldehyde by adding 7.0 $\mathrm{ml}$ of $37 \%$ formaldehyde and incubating them for 5 minutes. Subsequently, $51.4 \mathrm{ml}$ 4.5 M Tris was added to a final concentration of $750 \mathrm{mM}$ and incubated for 1 minute. The cells were harvested as described in the general methods section.

Abf1-aa cells were bead beated for 7x 3 minutes in a genie disruptor in the presence of protease inhibitors, either by addition of a protease inhibitor tablet per $25 \mathrm{ml} \mathrm{FA}$ lysis buffer (Roche \#11836145001) or by addition of $30 \mu \mathrm{l}$ aprotinin (Sigma-Aldrich: \#A6279), $1 \mu$ l leupeptin (Sigma-Aldrich \#L2884, $1 \mathrm{mg} / \mathrm{ml}$ in MQ), $1 \mu$ l pepstatin (Sigma-Aldrich \#P4265: $1 \mathrm{mg} / \mathrm{ml}$ in 100\% Methanol) and $15 \mu \mathrm{l}$ PMSF (Sigma-Aldrich: \#P7626, $200 \mathrm{mM}$ in isopropanol) per milliliter of FA lysis buffer. A $20 \mu \mathrm{l}$ sample was taken before lysis for Western blotting (pre-lysis). The cell debris was pelleted and the supernatant was used for sonication. A $40 \mu \mathrm{l}$ aliquot was taken for Western blotting (post-lysis). The chromatin was fragmented by splitting each sample over 2x $1.5 \mathrm{ml}$ sonication tubes and sonicating for 4 cycles 30 seconds on / 30 seconds off. For the samples with the separate protease inhibitors, $10 \mu \mathrm{l}$ was added right before the sonication, because PMSF loses activity in aqueous solutions. The sheared chromatin was pooled per cell pellet. A $40 \mu \mathrm{l}$ aliquot was taken for Western blotting (post-lysis) and $450 \mu \mathrm{l}$ of chromatin was used for each IP.

The IPs with magnetic beads were performed as described in the general methods section. $50 \mu \mathrm{l}$ magnetic beads and $1 \mu \mathrm{l}$ anti-V5 antibody were used for the IPs. The chromatin was incubated with the anti-V5 antibody for 2 hours. To the samples 
that were prepared with the separate protease inhibitors, again protease inhibitors were added: $15 \mu \mathrm{l}$ aprotinin, $0.5 \mu \mathrm{l}$ pepstatin $\mathrm{A}(1 \mathrm{mg} / \mathrm{ml}$ in $100 \%$ Methanol $), 0.5 \mu \mathrm{l}$ leupeptin ( $1 \mathrm{mg} / \mathrm{ml}$ in MQ) and $5 \mu \mathrm{PMSF}$ (200 mM in isopropanol). $50 \mu \mathrm{l}$ beads per IP was washed in individual $1.5 \mathrm{ml}$ Eppendorf tubes using $500 \mu \mathrm{l}$ PBS-T. The beads were resuspended in $400 \mu \mathrm{l}$ PBS, $25 \mu \mathrm{l}$ BSA $(10 \mathrm{mg} / \mathrm{ml}$ in TBS-T) was added and the beads were incubated at $4^{\circ} \mathrm{C}$ while the chromatin was incubating with the antibody. Just before this incubation was finished, the beads were washed again with $500 \mu \mathrm{l}$ PBS-T. The chromatin and antibody were added to the beads and incubated at RT for 20 minutes. To the samples that were prepared with the separate protease inhibitors, again $5 \mu \mathrm{l}$ PMSF was added. The beads were washed with the standard wash of $2 x$ PBS and 2x PBS-T and DNA was recovered by eluting overnight in TE/SDS at $65^{\circ} \mathrm{C}$. The IPs were performed in duplicate.

Samples for Western blotting were prepared by mixing $40 \mu \mathrm{l}$ of the sample with $10 \mu \mathrm{l}$ of $5 \mathrm{X}$ sample buffer (5\% SDS, $200 \mathrm{mM}$ Tris $\mathrm{pH} 6.8,25 \%$ glycerol, $1.43 \mathrm{M}$ $\beta$-mercaptoethanol, $0.032 \%$ bromophenol blue). Pre-lysis samples were prepared as described in the general methods section, by lysing the cells using $\mathrm{NaOH}$. All samples were heated to $95^{\circ} \mathrm{C}$ for 30 minutes to reverse cross-links. The samples $(10 \mu \mathrm{l})$ were separated on a $10 \%$ stain-free acrylamide gel (Bio-Rad \#1610182). As a control, a non-cross-linked crude lysate of a WT-aa was taken along, which was heated to $95^{\circ} \mathrm{C}$ for 5 minutes prior to loading. After the proteins had migrated through the gel, the proteins were transferred to a nitrocellulose membrane. The membrane was stained with an anti-V5 antibody (Life Technologies \#R96025) and binding was visualized using a goat-anti-mouse-HRP conjugated antibody (Bio-Rad \#1706516) and ECL solution, as described in the general methods section.

\section{Figure $8 \mathrm{C}$}

Cbf1-aa, Mcm1-aa, Reb1-aa and Sum1-aa strains were grown on a different day than the Abf1-aa strains used in Fig 8A, as $100 \mathrm{ml}$ cultures. The cells were cross-linked by adding $2.8 \mathrm{ml}$ of $37 \%$ formaldehyde to a final concentration of $1 \%$ and incubating them for 5 minutes. Subsequently, $20.6 \mathrm{ml}$ of $4.5 \mathrm{M}$ Tris $\mathrm{pH} 8.0$ was added to a final concentration of $750 \mathrm{mM}$ and the cells were incubated for 1 minute. Cells were harvested as described in the general methods section

The cells were bead beated for $7 \times 3$ minutes in a genie disruptor in the presence of protease inhibitors by addition of $30 \mu \mathrm{l}$ aprotinin (Sigma-Aldrich: \#A6279), $1 \mu \mathrm{l}$ leupeptin (Sigma-Aldrich \#L2884, $1 \mathrm{mg} / \mathrm{ml}$ in MQ), $1 \mu \mathrm{l}$ pepstatin (Sigma-Aldrich \#P4265: $1 \mathrm{mg} / \mathrm{ml}$ in 100\% Methanol) and $15 \mu \mathrm{l}$ PMSF (Sigma-Aldrich: \#P7626, 200 $\mathrm{mM}$ in isopropanol) per milliliter of FA lysis buffer. A $20 \mu \mathrm{l}$ sample was taken before lysis for Western blotting (pre-lysis). The cell debris was pelleted and the supernatant was used for sonication. A $40 \mu \mathrm{l}$ aliquot was taken for Western blotting (post-lysis). The chromatin was fragmented by splitting each sample over $2 \times 1.5 \mathrm{ml}$ sonication tubes and sonicating for 4 cycles 30 seconds on / 30 seconds off. $5 \mu$ PMSF was again added right before the sonication, because PMSF loses activity in aqueous solutions. The sheared chromatin was pooled per cell pellet. A $40 \mu \mathrm{l}$ aliquot was taken for Western blotting (post-lysis) and $450 \mu$ l of chromatin was used for each IP. 
The samples for Western blotting were prepared by mixing $40 \mu \mathrm{l}$ of the sample with $10 \mu \mathrm{l}$ of $5 \mathrm{X}$ sample buffer (5\% SDS, $200 \mathrm{mM}$ Tris $\mathrm{pH}$ 6.8, 25\% glycerol, $1.43 \mathrm{M}$ $\beta$-mercaptoethanol, $0.032 \%$ bromophenol blue). The pre-lysis samples were prepared as described in the general methods section, by lysing the cells using $\mathrm{NaOH}$. All samples were heated to $95^{\circ} \mathrm{C}$ for 30 minutes to reverse cross-links. The samples (10 $\mu \mathrm{l})$ were separated on a 10\% stain-free acrylamide gel (Bio-Rad \#1610182). As a control, a non-cross-linked crude lysate of a WT-aa was taken along, which was heated to $95^{\circ} \mathrm{C}$ for 5 minutes prior to loading. After the proteins had migrated through the gel, the proteins were transferred to a nitrocellulose membrane. The membrane was stained with an anti-V5 antibody (Life Technologies \#R96025) and binding was visualized using a goat-anti-mouse-HRP conjugated antibody (Bio-Rad $\# 1706516$ ) and ECL solution, as described in the general methods section.

\section{Figure 9}

Abf1-aa and Reb1-aa strains were grown as $100 \mathrm{ml}$ cultures. The cells were crosslinked for 5 minutes using a final concentration of $1 \%$, $2 \%$ or $3 \%$ formaldehyde, by

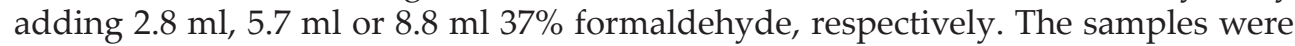
subsequently quenched with a final concentration of $750 \mathrm{mM}$ ( $1 \%$ formaldehyde), $1.5 \mathrm{M}(2 \%$ and $3 \%$ formaldehyde) or $2.0 \mathrm{M}$ ( $3 \%$ formaldehyde) by adding $20.6 \mathrm{ml}$ (1\% formaldhyde, $750 \mathrm{mM}$ Tris), 52.9 (2\% formaldehyde, $1.5 \mathrm{mM}$ Tris), 54.4 (3\% formaldehyde, $1.5 \mathrm{mM}$ Tris) or $87.1 \mathrm{ml}$ (3\% formaldehyde, $2.0 \mathrm{mM}$ Tris) 4.5M Tris $\mathrm{pH}$ 8.0 and incubating the samples for 1 minute. The cells were harvested as described in the general methods section

The cells were bead beated for $7 \times 3$ minutes in a genie disruptor in the presence of protease inhibitors by addition of $30 \mu \mathrm{l}$ aprotinin (Sigma-Aldrich: \#A6279), $1 \mu \mathrm{l}$ leupeptin (Sigma-Aldrich \#L2884, $1 \mathrm{mg} / \mathrm{ml}$ in MQ), $1 \mu \mathrm{l}$ pepstatin (Sigma-Aldrich \#P4265: $1 \mathrm{mg} / \mathrm{ml}$ in 100\% Methanol) and $15 \mu \mathrm{l}$ PMSF (Sigma-Aldrich: \#P7626, 200 $\mathrm{mM}$ in isopropanol) per milliliter of FA lysis buffer. A $5 \mu \mathrm{l}$ sample was taken before lysis for Western blotting (pre-lysis). The cell debris was pelleted and the supernatant was used for sonication. A $10 \mu \mathrm{l}$ aliquot was taken for Western blotting (post-lysis). The chromatin was fragmented by splitting each sample over $2 \times 1.5 \mathrm{ml}$ sonication tubes and sonicating for 10 cycles 30 seconds on / 15 seconds off. $5 \mu$ of PMSF was again added right before the sonication, because PMSF loses activity in aqueous solutions. The sheared chromatin was pooled per cell pellet. A $10 \mu \mathrm{l}$ aliquot was taken for Western blotting (post-lysis) and $450 \mu \mathrm{l}$ of chromatin was used for each IP.

The IPs with magnetic beads were performed as described in the general methods section. $25 \mu \mathrm{l}$ magnetic beads and $1 \mu \mathrm{l}$ anti-V5 antibody were used for the IPs. The chromatin was incubated with the anti-V5 antibody for 2 hours. Before the incubation, again protease inhibitors were added: $0.5 \mu$ l pepstatin, $0.5 \mu l$ leupeptin, $15 \mu \mathrm{l}$ aprotinin and $5 \mu \mathrm{l}$ PMSF. $25 \mu \mathrm{l}$ beads per IP was washed in individual $1.5 \mathrm{ml}$ Eppendorf tubes using $500 \mu \mathrm{l}$ PBS-T. The beads were resuspended in $400 \mu \mathrm{l}$ PBS, $25 \mu \mathrm{l} \mathrm{BSA}\left(10 \mathrm{mg} / \mathrm{ml}\right.$ in TBS-T) was added and the beads were incubated at $4^{\circ} \mathrm{C}$ while the chromatin was incubating with the antibody. Just before this incubation 
was finished, the beads were washed again with $500 \mu \mathrm{l}$ PBS-T. The chromatin and antibody were added to the beads and incubated at RT for 20 minutes. Right before the incubation of the chromatin and antibody with the beads, again $5 \mu$ PMSF was added. The beads were washed with the standard wash of 2x PBS and 2x PBS-T and DNA was recovered by eluting overnight in TE/SDS at $65^{\circ} \mathrm{C}$. The IPs were performed without replicates.

The samples for Western blotting were prepared by mixing $10 \mu \mathrm{l}$ of the sample with $2.5 \mu$ l of 5 X sample buffer (5\% SDS, $200 \mathrm{mM}$ Tris $\mathrm{pH} 6.8,25 \%$ glycerol, $1.43 \mathrm{M}$ $\beta$-mercaptoethanol, $0.032 \%$ bromophenol blue). The pre-lysis samples were prepared as described in the general methods section, by lysing the cells using $\mathrm{NaOH}$. All samples were heated to $95^{\circ} \mathrm{C}$ for 30 minutes to reverse cross-links. The samples (10 $\mu \mathrm{l})$ were separated on a 10\% stain-free acrylamide gel (Bio-Rad \#1610182). As a control, a non-cross-linked crude lysate of a WT-aa was taken along, which was heated to $95^{\circ} \mathrm{C}$ for 5 minutes prior to loading. After the proteins had migrated through the gel, the proteins were transferred to a nitrocellulose membrane. The membrane was stained with an anti-V5 antibody (Life Technologies \#R96025) and binding was visualized using a goat-anti-mouse-HRP conjugated antibody (Bio-Rad \#1706516) and ECL solution, as described in the general methods section.

\section{Supplemental Tables}

Table S1 List of genotypes of strains used in this study

\begin{tabular}{|c|c|}
\hline Strain & Genotype \\
\hline WT-aа & tor1-1; $\triangle$ fpr1; RPL13-2xFKBP12-NATMX6; MET15; his3-1; leu2; lys2; ura3; MATalpha \\
\hline Cbf1-aa & $\begin{array}{l}\text { tor1-1; } \triangle \text { fpr1; RPL13-2xFKBP12-NATMX6; MET15; his3-1; leu2; lys2; ura3; CBF1- } \\
\text { FRB-yEGFP-3V5-hphMX6; MATalpha }\end{array}$ \\
\hline $\begin{array}{l}\text { Cbf1-aa } \\
\text { Cha4-V5 }\end{array}$ & $\begin{array}{l}\text { tor1-1; } \triangle \text { fpr1; RPL13-2xFKBP12-NATMX6; MET15; his3-1; leu2; lys2; ura3; CHA4- } \\
\text { 3V5-bleMX6; CBF1-FRB-yEGFP-3V5-hphMX6 MATalpha }\end{array}$ \\
\hline Abf1-aa & $\begin{array}{l}\text { tor1-1; } \triangle \text { fpr1; RPL13-2xFKBP12-NATMX6; MET15; his3-1; leu2; lys2; ura3; ABF1- } \\
\text { FRB-yEGFP-3V5-hphMX6; MATalpha }\end{array}$ \\
\hline Reb1-aa & $\begin{array}{l}\text { tor1-1; } \triangle \text { fpr1; RPL13-2xFKBP12-NATMX6; MET15; his3-1; leu2; lys2; ura3; REB1- } \\
\text { FRB-yEGFP-3V5-hphMX6; MATalpha }\end{array}$ \\
\hline Mcm1-aа & $\begin{array}{l}\text { tor1-1; } \triangle \text { fpr1; RPL13-2xFKBP12-NATMX6; MET15; his3-1; leu2; lys2; ura3; MCM1- } \\
\text { FRB-yEGFP-3V5-hphMX6; MATalpha }\end{array}$ \\
\hline Sum1-aa & $\begin{array}{l}\text { tor1-1; } \triangle \text { fpr1; RPL13-2xFKBP12-NATMX6; MET15; his3-1; leu2; lys2; ura3; SUM1- } \\
\text { FRB-yEGFP-3V5-hphMX6; MATalpha }\end{array}$ \\
\hline
\end{tabular}


Table S2 list of primers used in this study

\begin{tabular}{|c|c|c|c|}
\hline Name & Sequence $\left(5^{\prime}->3^{\prime}\right)$ & Target & Figure \\
\hline ACT1_nucl-F & ATATGTTTAGAGGTTGCTGCTTTG & \multirow{2}{*}{ Background } & \multirow{2}{*}{ 2A-2D } \\
\hline ACT1_nucl-R & AACCGGCTTTACACATACCA & & \\
\hline BNA2_qP_F & TTTCTCTATGGGCTGACG & \multirow{2}{*}{ Sum1 } & \multirow{2}{*}{7} \\
\hline BNA2_qP_R & ATGCTAAAGATACATGGACATC & & \\
\hline CFD1-F & CGGGATCTTTGGTTCCTATC & \multirow{2}{*}{ Background } & \multirow{2}{*}{$2 \mathrm{E}$} \\
\hline CFD1-R & AGCCTTCCGATTTCTTTCC & & \\
\hline CHA1-F & GGGCGGCTCCTGTTAAG & \multirow{2}{*}{ Cha4 } & \multirow{2}{*}{$3,4 \mathrm{~B}, 5$} \\
\hline CHA1-R & TCCTCCTCATATTGTCCСTTT & & \\
\hline CPA2_TF_F & CACAATCGTTACGACATGGAG & \multirow{2}{*}{ Background } & \multirow{2}{*}{$2 \mathrm{E}, 3,4 \mathrm{~B}, 5 \mathrm{~A}$} \\
\hline CPA2_TF_R & GACTCTTATTGATGAGATGGCAATA & & \\
\hline EMC6_qP_F & TACGGTCACGCCAATTC & \multirow{2}{*}{ Reb1 } & \multirow{2}{*}{$9 \mathrm{~B}$} \\
\hline EMC6_qP_R & GCTTGAGCAATCCAACATAAG & & \\
\hline FCF1_qP_F & ACTATCGGTTCCTACTGGAAGA & \multirow{2}{*}{ Abf1 } & \multirow{2}{*}{$8,9 \mathrm{~A}$} \\
\hline FCF1_qP_R & GGCATCATTCAAGAATAGTAGCAAG & & \\
\hline GLN1_qP_F & TACCCGCATACGGTTCT & \multirow{2}{*}{ Reb1 } & \multirow{2}{*}{$9 \mathrm{~B}$} \\
\hline GLN1_qP_R & GGAGCGCAGTCATCAAT & & \\
\hline QCR10-F & TAACGCTGTCGCACTTTGA & \multirow{2}{*}{ Cbf1 } & \multirow{2}{*}{ 2A-2D, 3-6 } \\
\hline QCR10-R & GAAACAACGGGTTGAACCATATT & & \\
\hline NHX1_qP_F & CACAAACGTGATAGCAAGGAAC & \multirow{2}{*}{ Abf1 } & \multirow{2}{*}{$8,9 \mathrm{~A}$} \\
\hline NHX1_qP_R & TCTTGGGTGCCCTTTATCTTAG & & \\
\hline TFC1_pr_qP_F & TCTTTAAGCTCTGCTGTGTT & \multirow{2}{*}{ Background } & \multirow{2}{*}{$6-9$} \\
\hline TFC1_pr_qP_R & AGCGAAGAAGCGAAGAAG & & \\
\hline TNA1_qP_F & АСТCTCCAAGCTATAAGCATAC & \multirow{2}{*}{ Sum1 } & \multirow{2}{*}{7} \\
\hline TNA1_qP_R & TTTCAGTCGCTGTCTCAC & & \\
\hline TUB1_pr_F & TACAGATCTTGGGTGGCGAGAACT & \multirow{2}{*}{ Background } & \multirow{2}{*}{ 2A-2D, 4A, 5B, 6-8 } \\
\hline TUB1_pr_R & AAACGCCTCGAGCCAAGGGAAA & & \\
\hline YOS1-F & GCTCGTAATGTCTCGAAATTTGTC & \multirow{2}{*}{ Cbf1 } & \multirow{2}{*}{$2-6$} \\
\hline YOS1-R & СTTATTGAGAAGGCTCCCAGTC & & \\
\hline
\end{tabular}




\section{Chapter 3}

Table 3 list of buffers used in this study

\begin{tabular}{|c|c|}
\hline Solution name & Components \\
\hline Formaldehyde & 37\% formaldehyde containing 10-15\% methanol (Sigma-Aldrich \#252549) \\
\hline Glycine & 2.5 M Glycine \\
\hline Tris & $4.5 \mathrm{M}$ Tris $\mathrm{pH} 8.0$ \\
\hline Buffer Z & $\begin{array}{l}1 \text { M sorbitol } \\
50 \mathrm{mM} \text { Tris pH } 7.5\end{array}$ \\
\hline $\begin{array}{l}\text { Zymolyase } \\
\text { solution }\end{array}$ & $\begin{array}{l}\text { Buffer Z } \\
10 \mathrm{mM} \beta \text {-mercaptoethanol } \\
10 \mathrm{mg} / \mathrm{ml} \text { zymolyase (zymolyase } 20 \text { T MP biomedical \#08320921) }\end{array}$ \\
\hline TBS & $\begin{array}{l}150 \mathrm{mM} \mathrm{NaCl} \\
10 \mathrm{mM} \text { Tris pH } 7.5\end{array}$ \\
\hline FA lysis buffer & $\begin{array}{l}50 \mathrm{mM} \text { HEPES-KOH pH } 7.5 \\
150 \mathrm{mM} \mathrm{NaCl} \\
1 \mathrm{mM} \text { EDTA pH } 8.0 \\
1 \% \text { Triton X-100 } \\
0.1 \% \text { Na-deoxycholate } \\
0.1 \% \text { SDS }\end{array}$ \\
\hline Aprotinin & 5 - 10 TIU /ml (Sigma-Aldrich: \#A6279), \\
\hline Pepstatin A & $1 \mathrm{mg} / \mathrm{ml}$ leupeptin in 100\% methanol $(1.51 \mathrm{mM})$ (Sigma-Aldrich \#L2884) \\
\hline Leupeptin & $1 \mathrm{mg} / \mathrm{ml}$ pepstatin A in MQ (2.10 mM) (Sigma-Aldrich \#P4265) \\
\hline PMSF & 200 mM Phenylmethanesulfonyl fluoride (PMSF) in isopropanol (Sigma \#P7626) \\
\hline RNAse A/T1 & RNAse A 2mg/ml \& RNase T1 5000 U/ml mix (Thermo Scientific \#EN0551) \\
\hline Proteinase K & $10 \mu \mathrm{g} / \mu \mathrm{l}$ in TE $(10 \mathrm{mM}$ Tris $\mathrm{pH} 8,1 \mathrm{mM}$ EDTA $\mathrm{pH} 8)$ \\
\hline PBS & pH adjusted to 7.3 using $\mathrm{HCl}$ \\
\hline PBS-T & PBS $+0.02 \%$ Tween-20 \\
\hline Wash buffer 1 & $\begin{array}{l}\text { FA lysis buffer } \\
500 \mathrm{mM} \mathrm{NaCl} \text { (final concentration) }\end{array}$ \\
\hline Wash buffer 2 & $\begin{array}{l}10 \mathrm{mM} \text { Tris } \mathrm{pH} 8.0 \\
0.25 \mathrm{mM} \mathrm{LiCl} \\
1 \mathrm{mM} \text { EDTA pH } 8.0 \\
0.5 \% \text { Nonidet P-40 } \\
0.5 \% \text { Na-deoxycholate }\end{array}$ \\
\hline TE/SDS & $\begin{array}{l}10 \mathrm{mM} \text { Tris pH } 8.0 \\
1 \mathrm{mM} \text { EDTA pH } 8.0 \\
1 \% \text { SDS }\end{array}$ \\
\hline BSA & $10 \mathrm{mg} / \mathrm{ml}$ in TBS-T (150 mM NaCl, $10 \mathrm{mM}$ Tris pH 7.5, 0.05\% Tween-20) \\
\hline 2X sample buffer & $\begin{array}{l}2 \% \text { SDS } \\
80 \text { mM Tris pH } 6.8 \\
10 \% \text { glycerol } \\
572 \text { mM } \beta \text {-mercaptoethanol } \\
0.016 \% \text { bromophenol blue }\end{array}$ \\
\hline
\end{tabular}




\begin{tabular}{|l|l|}
\hline 5X sample buffer & $5 \%$ SDS \\
& $200 \mathrm{mM}$ Tris $\mathrm{pH} 6.8$ \\
& $25 \%$ glycerol \\
& $1.43 \mathrm{M} \beta$-mercaptoethanol \\
& $0.032 \%$ bromophenol blue \\
\hline
\end{tabular}




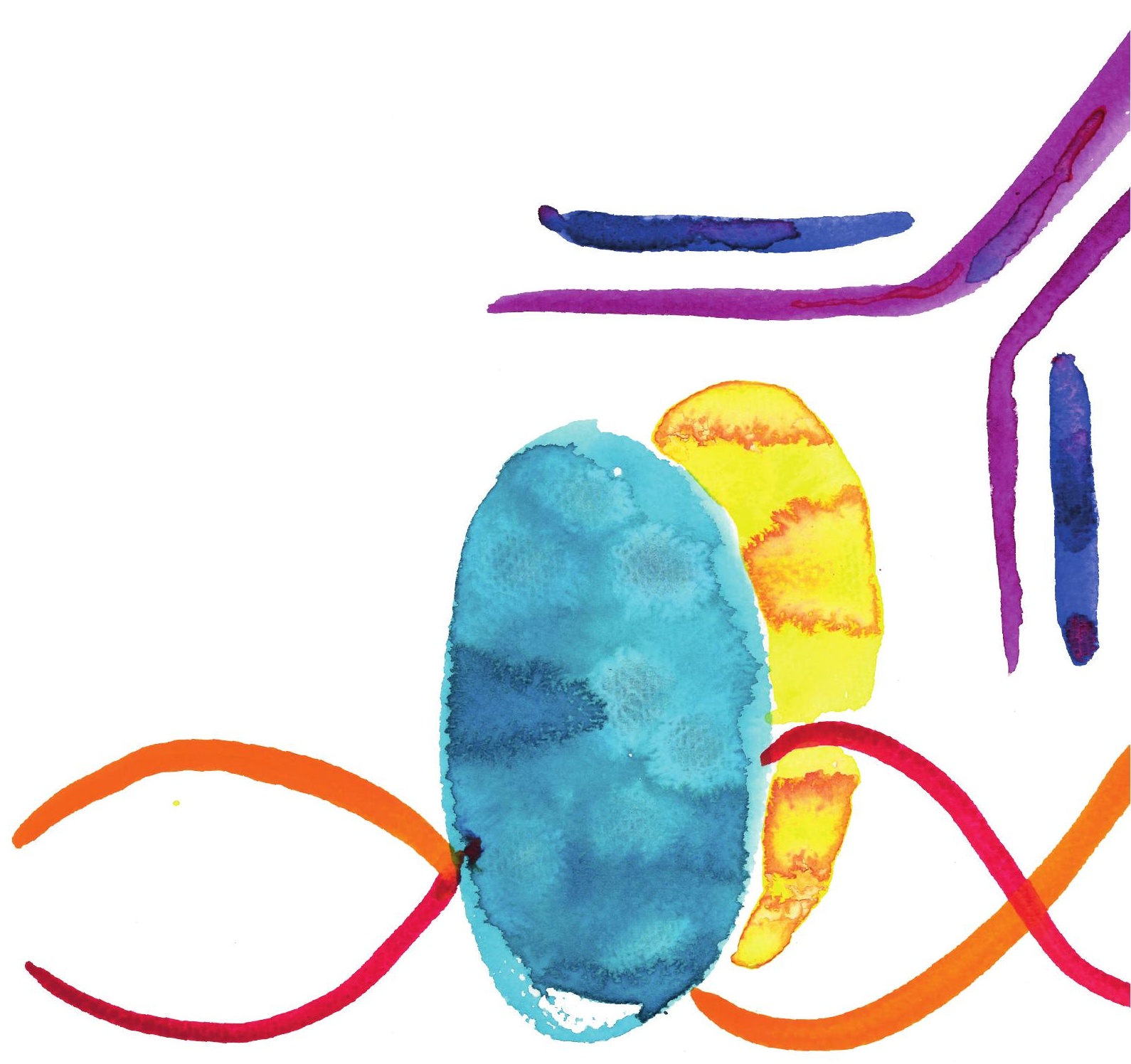




\section{Chapter 4}

An optimized chromatin immunoprecipitation protocol for quantification of protein-DNA interactions

Wim J. de Jonge, Mariël Brok, Patrick Kemmeren and Frank C.P. Holstege

Princess Máxima Center for Pediatric Oncology, Heidelberglaan 25, 3584 CS Utrecht, the Netherlands 


\section{Abstract}

Transcription factors are important regulators of cell fate and function. Knowledge about where transcription factors are bound in the genome is crucial for understanding their function. A common method to study protein-DNA interactions is chromatin immunoprecipitation (ChIP). Here, we present a revised ChIP protocol to determine protein-DNA interactions for the yeast Saccharomyces cerevisiae. We optimized several aspects of the procedure including cross-linking and quenching, cell lysis and immunoprecipitation steps. This protocol facilitates sensitive and reproducible quantitation of protein-DNA interactions.

For complete details on the use and execution of this protocol, please refer to (de Jonge et al, 2019). 


\section{Before you begin}

Timing: $0.5-4$ hours

1. If needed, prepare buffers. Make sure that there is enough of all solutions that are needed before cell growth, especially the $4.5 \mathrm{M}$ Tris $\mathrm{pH}$ 8.0. Per sample $\sim 55$ $\mathrm{ml}$ 4.5M Tris $\mathrm{pH} 8.0$ is needed to properly quench the formaldehyde.

2. Make sure to use fresh formaldehyde that is $<3$ months old, preferably $<1$ month.

3. 4 days before the start of the experiment (day -3), streak the strains of interest on appropriate selection plates and incubate for 3 days @ $30^{\circ} \mathrm{C}$.

Note: If possible, use a strain that has an epitope tagged protein of interest (e.g. HA, FLAG, V5, etc). High quality antibodies are available for these tags, which makes the immunoprecipitation (IP) more efficient. This protocol was optimized for the use of a V5 tag and an anti-V5 antibody, nevertheless, it should be applicable for other antibodies as well. Optimal incubation conditions may differ between antibodies.

4. The day before the start of the experiment (day 0 ), in the morning, pick at least three colonies for each strain/condition from a fresh plate and inoculate each colony in $1.5 \mathrm{ml}$ medium in a 24-well plate. Grow the cultures while shaking $(230 \mathrm{rpm})$ at $30^{\circ} \mathrm{C}$. At the end of the day, combine each well $(1.5 \mathrm{ml})$ with $13.5 \mathrm{ml}$ $30^{\circ} \mathrm{C}$ for a culture of $15 \mathrm{ml}$ in a $100 \mathrm{ml}$ flask and grow for 12-16 hours.

Note: All incubations are performed at $30^{\circ} \mathrm{C}$ and $230 \mathrm{rpm}$.

Note: We use SC medium for all experiments, but any other appropriate medium can be used as well.

Note: If a larger volume of starting culture is required on day 1 , it is possible to combine three $1.5 \mathrm{ml}$ cultures to make a culture of $20 \mathrm{ml}$ by adding $15.5 \mathrm{ml}$ of medium. 
Key resources table

\begin{tabular}{|c|c|c|}
\hline Reagent or Resource & Source & Identifier \\
\hline \multicolumn{3}{|l|}{ Antibodies } \\
\hline $\begin{array}{l}\text { V5 Tag monoclonal Antibody IP grade } \\
\text { (mouse) }\end{array}$ & Thermo Fisher Scientific & Cat\# R960-25, RRID:AB_2556564 \\
\hline $\begin{array}{l}\text { Goat Anti-Mouse IgG (H L)-HRP } \\
\text { Conjugate antibody }\end{array}$ & Bio-Rad & $\begin{array}{l}\text { Cat\# 170-6516, } \\
\text { RRID:AB_11125547 }\end{array}$ \\
\hline \multicolumn{3}{|c|}{ Chemicals, Peptides, and Recombinant Proteins } \\
\hline $\begin{array}{l}\text { Yeast Nitrogen Base w/o AA, } \\
\text { Carbohydrate \& w/AS (YNB) (Powder) }\end{array}$ & US Biological & Cat\#Y2025 \\
\hline $37 \%$ formaldehyde & Sigma-Aldrich & Cat\#252549 \\
\hline $\begin{array}{l}\text { UltraPure }{ }^{\mathrm{TM}} \text { Tris Buffer (powder } \\
\text { format) }\end{array}$ & Invitrogen & Cat\#15504020 \\
\hline Zymolyase $20 \mathrm{~T}$ & MP biomedical & Cat\#08320921 \\
\hline Aprotinin & Sigma-Aldrich & Cat\#A6279 \\
\hline Pepstatin A & Sigma-Aldrich & Cat\#P4265-1MG \\
\hline Leupeptin & Sigma-Aldrich & Cat\#L2884-1MG \\
\hline PMSF & Sigma-Aldrich & Cat\#P7626 \\
\hline Proteinase K & Roche & Cat\#03115852001 \\
\hline Broad Range Protein Marker & New England Biolabs & Cat\#P7708 \\
\hline Dynabeads Protein G & Thermo Fisher Scientific & Cat\#10003D \\
\hline RNAse A/T1 & Thermo Fisher Scientific & Cat\#EN0551 \\
\hline \multicolumn{3}{|l|}{ Critical Commercial Assays } \\
\hline QIAquick PCR Purification Kit & Qiagen & Cat\#28106 \\
\hline $\begin{array}{l}\text { TGX Stain-Free }{ }^{\mathrm{TM}} \text { FastCast }^{\mathrm{TM}} \\
\text { Acrylamide Kit }(10 \%)\end{array}$ & Bio-Rad & Cat\#1610182 \\
\hline $\begin{array}{l}\text { Western Lightning Plus-ECL, Enhanced } \\
\text { Chemiluminescence Substrate }\end{array}$ & Perkin Elmer & Cat\#NEL105001EA \\
\hline High Sensitivity DNA Kit & Agilent & Cat\#5067-4626 \\
\hline IQ SYBR Green super mix & Bio-Rad & Cat\#1708886 \\
\hline Precision Blue $^{\mathrm{TM}}$ Real-Time PCR Dye & Bio-Rad & Cat\#1725555 \\
\hline \multicolumn{3}{|l|}{ Software and Algorithms } \\
\hline ImageJ software & Schneider et al, 2012 & https://imagej.nih.gov/ij/ \\
\hline CFX Maestro software & Bio-Rad & Cat\#12004110 \\
\hline \multicolumn{3}{|l|}{ Other } \\
\hline Cell Density Meter & VWR & Cat\#634-0882 \\
\hline Heated magnetic stir plate C-MAG HS 7 & IKA & Cat $\# 0003581200$ \\
\hline Zirconium/silica beads $0.5 \mathrm{~mm}$ & BioSpec Products & Cat $\# 11079105 z$ \\
\hline Analog Disruptor Genie & $\begin{array}{l}\text { Scientific Industries/ } \\
\text { VWR }\end{array}$ & Cat\#444-0943 \\
\hline
\end{tabular}




\begin{tabular}{|c|c|c|}
\hline $\begin{array}{l}\text { BD Microlance Hypod. naald - 23G } 1 \text { - } \\
0,6 \times 25 \mathrm{~m}\end{array}$ & Becton Dickinson & Cat $\# 300800$ \\
\hline $\begin{array}{l}\text { Cooled micro centrifuge for } 1.5-2.0 \mathrm{ml} \\
\text { tubes }\end{array}$ & N/A & N/A \\
\hline $\begin{array}{l}\text { Cooled tabletop centrifuge for } 50 \mathrm{ml} \\
\text { tubes }\end{array}$ & N/A & N/A \\
\hline Vortex & $\mathrm{N} / \mathrm{A}$ & N/A \\
\hline Bioruptor pico microtubes & Diagenode & Cat\#C30010016 \\
\hline Bioruptor pico sonicator device & Diagenode & Cat\#B01060010 \\
\hline Thermoshaker (preferred) or heat block & $\mathrm{N} / \mathrm{A}$ & N/A \\
\hline $\begin{array}{l}\text { Rotating wheel (one at } 4^{\circ} \mathrm{C} \text { and one at } \\
19-22^{\circ} \mathrm{C} \text { ) }\end{array}$ & N/A & N/A \\
\hline Bioanalyzer & Agilent & Cat\#G2939B \\
\hline DynaMag-2 magnetic stand & Thermo Fisher Scientific & Cat\#12321D \\
\hline $1.5 \mathrm{ml}$ DNA LoBind Tube & Eppendorf & Cat\#0030108051 \\
\hline CoolRack XT PCR384 for 384-wel & Corning & Cat\#432055 \\
\hline $\begin{array}{l}\text { Hard-Shell@ Thin-Wall 384-Well Skirted } \\
\text { PCR Plates, white }\end{array}$ & Bio-Rad & Cat\#HSP3805 \\
\hline Microseal® ‘B’ PCR Plate Sealing Film & Bio-Rad & Cat\#msb1001 \\
\hline $\begin{array}{l}\text { CFX384 Touch Real-Time PCR } \\
\text { Detection system }\end{array}$ & Bio-Rad & Cat\#698-2901 \\
\hline
\end{tabular}

\section{Materials and equipment}

Alternatives: This protocol uses a Bioanalyzer to assess the extent of DNA fragmentation. Alternatives are for example the TapeStation (Agilent) or LabChip (PerkinElmer). The TapeStation offers more flexibility in the number of samples that can be analyzed at once and the LabChip can process more samples at the same time. The use of a Bioanalyzer (or equivalent) is preferred over an agarose gel since the Bioanalyzer has a better resolution in assessing the exact fragmentation pattern. An agarose gel can distinguish large differences in shearing (500-1000 bp fragments vs $200-300$ bp fragments), but with an agarose gel it is hard to distinguish between more subtle differences (200 - 300 bp fragments vs $300-400$ bp fragments). In addition, the Bioanalyzer (or equivalent) is also more sensitive and only requires a small fraction of material compared to what is needed to visualize fragmentation using an agarose gel. Nevertheless, if analysis by Bioanalyzer (or equivalent) is not possible, the fragmentation can also be assessed using agarose gel electrophoresis.

Alternatives: This protocol describes the qPCR procedure using a CFX384 Touch Real-Time PCR Detection system and corresponding reagents, in $10 \mu$ reaction volumes. Any other qPCR machine can be used as well. We recommend using a 384well format, since this allows examination of multiple IP-input pairs with several primer pairs in the same plate. 


\section{FA lysis buffer}

Preparation of FA lysis buffer. We filter sterilize the FA lysis buffer. Store at $4^{\circ} \mathrm{C}$. Right before use, add $30 \mu \mathrm{l}$ Aprotinin, $1 \mu \mathrm{l}$ Pepstatin, $1 \mu \mathrm{l}$ Leupeptin and $10 \mu \mathrm{l}$ PMSF per $1 \mathrm{ml}$ of buffer (see also the table with other solutions required for the ChIP protocol).

\begin{tabular}{|l|l|l|l|}
\hline Reagent & Final concentration & Stock concentration & Add to $500 \mathbf{~ m l}$ \\
\hline HEPES-KOH pH 7.5 & $50 \mathrm{mM}$ & $1 \mathrm{M}$ & $25 \mathrm{ml}$ \\
\hline $\mathrm{NaCl}$ & $150 \mathrm{mM}$ & $5 \mathrm{M}$ & $15 \mathrm{ml}$ \\
\hline EDTA pH 8.0 & $1 \mathrm{mM}$ & $0.5 \mathrm{M}$ & $1 \mathrm{ml}$ \\
\hline Triton X-100 & $1 \%(\mathrm{v} / \mathrm{v})$ & $10 \%(\mathrm{v} / \mathrm{v})$ & $50 \mathrm{ml}$ \\
\hline Na-deoxycholate & $0.1 \%(\mathrm{w} / \mathrm{v})$ & $10 \%(\mathrm{w} / \mathrm{v})$ & $5 \mathrm{ml}$ \\
\hline SDS & $0.1 \%(\mathrm{w} / \mathrm{v})$ & $10 \%(\mathrm{w} / \mathrm{v})$ & $5 \mathrm{ml}$ \\
\hline MilliQ & & & $399 \mathrm{ml}$ \\
\hline
\end{tabular}

\subsection{Tris $\mathrm{pH} 8.0$}

Preparation of 4.5M Tris $\mathrm{pH}$ 8.0. We filter sterilize the 4.5 M Tris buffer, although autoclaving should also be possible. Store at $19-22^{\circ} \mathrm{C}$.

\begin{tabular}{|l|l|l|}
\hline Reagent & Stock concentration & Add to 1 L \\
\hline Tris & - & $545.13 \mathrm{~g}$ \\
\hline $\mathrm{HCl}$ & $12.1 \mathrm{M}$ & $\sim 211.5 \mathrm{ml}$ \\
\hline MilliQ & & Up to $1 \mathrm{~L}$ \\
\hline
\end{tabular}

Note: Making 4.5M Tris pH 8.0 is challenging, because this concentration nearly reaches the solubility limit of Tris. We recommend making 2 liters at once. For each $100 \mathrm{ml}$ culture, cross-linked with $2 \%$ formaldehyde, almost $55 \mathrm{ml}$ of $4.5 \mathrm{M}$ Tris $\mathrm{pH} 8.0$ is used to quench (final concentration: $1.5 \mathrm{M}$ ). This means that to quench 18 samples, nearly 1 liter of $4.5 \mathrm{M}$ Tris $\mathrm{pH} 8.0$ is needed. When dissolving Tris, a substantial amount of $\mathrm{HCl}$ must be added to fully dissolve Tris ( $211.5 \mathrm{ml}$ per liter), which should be taken into account when adding MQ.

\section{ChIP wash buffer 1}

Preparation of ChIP wash buffer 1 (optional). Store at $19-22^{\circ} \mathrm{C}$. Preferably add right before use $30 \mu \mathrm{l}$ Aprotinin, $1 \mu \mathrm{l}$ Pepstatin, $1 \mu \mathrm{l}$ Leupeptin and $10 \mu \mathrm{l}$ PMSF per $1 \mathrm{ml}$ of buffer.

\begin{tabular}{|l|l|l|l|}
\hline Reagent & Final concentration & Stock concentration & Add to 50 ml \\
\hline HEPES-KOH pH 7.5 & $50 \mathrm{mM}$ & $1 \mathrm{M}$ & $2.5 \mathrm{ml}$ \\
\hline $\mathrm{NaCl}$ & $500 \mathrm{mM}$ & $5 \mathrm{M}$ & $5 \mathrm{ml}$ \\
\hline EDTA pH 8.0 & $1 \mathrm{mM}$ & $0.5 \mathrm{M}$ & $0.1 \mathrm{ml}$ \\
\hline Triton X-100 & $1 \%(\mathrm{v} / \mathrm{v})$ & $10 \%(\mathrm{v} / \mathrm{v})$ & $5 \mathrm{ml}$ \\
\hline Na-deoxycholate & $0.1 \%(\mathrm{w} / \mathrm{v})$ & $10 \%(\mathrm{w} / \mathrm{v})$ & $0.5 \mathrm{ml}$ \\
\hline SDS & $0.1 \%(\mathrm{w} / \mathrm{v})$ & $10 \%(\mathrm{w} / \mathrm{v})$ & $0.5 \mathrm{ml}$ \\
\hline MilliQ & & & $36.4 \mathrm{ml}$ \\
\hline
\end{tabular}


ChIP wash buffer 2

Preparation of ChIP wash buffer 2 (optional). Store at $19-22^{\circ} \mathrm{C}$. Preferably add $30 \mu \mathrm{l}$ Aprotinin, $1 \mu \mathrm{l}$ Pepstatin, $1 \mu \mathrm{l}$ Leupeptin and $10 \mu \mathrm{l}$ PMSF per $1 \mathrm{ml}$ of buffer, right before use.

*Nonidet P-40 is discontinued. Substitutes are available, but we did not test these.

\begin{tabular}{|l|l|l|l|}
\hline Reagent & Final concentration & Stock concentration & Add to 50 ml \\
\hline Tris pH 8.0 & $10 \mathrm{mM}$ & $1 \mathrm{M}$ & $0.5 \mathrm{ml}$ \\
\hline $\mathrm{LiCl}$ & $250 \mathrm{mM}$ & $5 \mathrm{M}$ & $2.5 \mathrm{ml}$ \\
\hline Nonidet P-40* & $0.5 \%(\mathrm{v} / \mathrm{v})$ & $10 \%(\mathrm{v} / \mathrm{v})$ & $2.5 \mathrm{ml}$ \\
\hline Na-deoxycholate & $0.5 \%(\mathrm{w} / \mathrm{v})$ & $10 \%(\mathrm{w} / \mathrm{v})$ & $2.5 \mathrm{ml}$ \\
\hline EDTA pH 8.0 & $1 \mathrm{mM}$ & $0.5 \mathrm{M}$ & $0.1 \mathrm{ml}$ \\
\hline MilliQ & & & $41.9 \mathrm{ml}$ \\
\hline
\end{tabular}

\section{Other solutions}

Other solutions required for the ChIP protocol

\begin{tabular}{|c|c|}
\hline Name & Reagents \\
\hline TBS & $150 \mathrm{mM} \mathrm{NaCl}, 10 \mathrm{mM}$ Tris pH 7.5 \\
\hline Aprotinin & 3-21 TIU/mL as purchased from supplier. Store at $4^{\circ} \mathrm{C}$. \\
\hline Pepstatin A & $1 \mathrm{mg} / \mathrm{ml}$ pepstatin A in $100 \%$ methanol $(1.51 \mathrm{mM})$. Store at $-20^{\circ} \mathrm{C}$. \\
\hline Leupeptin & $1 \mathrm{mg} / \mathrm{ml}$ leupeptin in MQ $(2.10 \mathrm{mM})$. Store at $-20^{\circ} \mathrm{C}$. \\
\hline PMSF & $\begin{array}{l}200 \mathrm{mM} \text { Phenylmethanesulfonyl fluoride (PMSF) in isopropanol. Heat to } 37^{\circ} \mathrm{C} \text { to } \\
\text { dissolve and store at }-20^{\circ} \mathrm{C} \text {. }\end{array}$ \\
\hline Proteinase K & $10 \mu \mathrm{g} / \mu \mathrm{l}$ in TE $(10 \mathrm{mM}$ Tris $\mathrm{pH} 8.0,1 \mathrm{mM}$ EDTA pH 8.0) \\
\hline PBS & $\begin{array}{l}137 \mathrm{mM} \mathrm{NaCl}, 2.7 \mathrm{mM} \mathrm{KCl}, 10 \mathrm{mM} \mathrm{Na} 2 \mathrm{HPO} 4,1.47 \mathrm{mM} \mathrm{KH} 2 \mathrm{PO} 4,1 \mathrm{mM} \mathrm{CaCl}, 0.5 \\
\mathrm{mM} \mathrm{MgCl} \text {, pH adjusted to } 7.3 \text { using } \mathrm{HCl}\end{array}$ \\
\hline PBS-T & PBS $+0.02 \%$ Tween-20 (v/v) \\
\hline TE/SDS & 10 mM Tris pH 8.0, 1 mM EDTA pH 8.0, 1\% SDS (w/v) \\
\hline BSA in TBS-T & Dissolved to $10 \mathrm{mg} / \mathrm{ml}$ in TBS-T (150 mM NaCl, $10 \mathrm{mM}$ Tris $\mathrm{pH} 7.5,0.05 \%$ Tween-20) \\
\hline $\begin{array}{l}\text { 5X sample } \\
\text { buffer } \\
\text { (optional) }\end{array}$ & $\begin{array}{l}5 \% \text { SDS }(\mathrm{w} / \mathrm{v}), 200 \mathrm{mM} \text { Tris } \mathrm{pH} 6.8,25 \% \text { glycerol }(\mathrm{v} / \mathrm{v}), 1.43 \mathrm{M} \beta \text {-mercaptoethanol, } \\
0.032 \% \text { bromophenol blue }(\mathrm{w} / \mathrm{v})\end{array}$ \\
\hline
\end{tabular}




\section{Step-by-step method details}

\section{Growth and in vivo cross-linking - Day 1}

Timing: $6-8$ hours

Cells are grown and cross-linked using formaldehyde to trap protein-DNA interactions.

Note: This protocol is an optimization of a previous protocol (Aparicio et al, 2004).

Note: Use an appropriate number of replicates. Since there are many steps in the protocol, the chances of variation arising among samples are substantial. We recommend to always use at least three biological replicates, if possible. In case of a tagged protein, the ideal biological replicates are independently transformed strains. These should be independently streaked and grown. If not available, different colonies should be grown independently as biological replicates.

Note: To control for artefactual binding of the protein of interest, take along a noncross-linking sample. If there is no binding observed in this control, this indicates that there is no artefactual binding of the protein of interest during the procedure. Treat this culture exactly the same as the cross-linked samples but omit the formaldehyde addition step. This control should be taken along every first time a new protein of interest is tested. When using tagged strains, it is also good practice to take along an untagged (wild-type) control.

1. Measure the optical density (OD) of the culture (1:50) and dilute the cultures in $110 \mathrm{ml}$ pre warmed medium to an OD of $0.20\left(\sim 0.5 \times 10^{7}\right.$ cells $\left./ \mathrm{ml}\right)$ in a $500 \mathrm{ml}$ Erlenmeyer, mix well and measure the starting OD of the culture.

Note: There is an extra $10 \mathrm{ml}$ for sampling OD. Before the addition of formaldehyde, remove what is left of this, to make sure that there is exactly $100 \mathrm{ml}$ culture left.

Note: Since timing of formaldehyde cross-linking is crucial, and this is performed using magnetic stir plates, it is best to start the cultures in such a way that only a few samples are ready at the same time. If, for example, a time course experiment is performed with replicates, having 1 hour in between each replicate time course will provide enough time to finish harvesting the first, before the second has to be processed. We strongly recommend harvesting the cultures with two people at the same time to speed up the process.

2. Grow yeast until OD 0.8 ( 2 doublings), this is equivalent to approximately $2 \mathrm{x}$ $10^{7}$ cells $/ \mathrm{ml}$.

3. Before addition of formaldehyde, put the cultures on a heated $\left(30^{\circ} \mathrm{C}\right)$ stir plate and add a (clean!) magnetic stir bar. Add $5.7 \mathrm{ml}$ of $37 \%$ formaldehyde to a final concentration of $2 \%$ to the cultures. Incubate outside the incubator on the heated $\left(30^{\circ} \mathrm{C}\right)$ stir plate under agitation/stirring for $5 \mathrm{~min}$. 
Note: Use a setting for the stir plate that allows for quick and proper mixing. Be careful not to mix too vigorously, however, especially avoiding foaming of the sample. For example, with the stir plate IKA C-MAG HS 7 and a stir bar of approximately $6 \mathrm{~cm}$, set the motility to 1 .

Note: It is useful to make aliquots (in $50 \mathrm{ml}$ tubes) of formaldehyde and $4.5 \mathrm{M}$ Tris $\mathrm{pH} 8.0$ in advance. The correct volume of formaldehyde and 4.5M Tris $\mathrm{pH} 8.0$ can be easily poured into the culture. Be careful to pour straight into the culture without touching the walls of the Erlenmeyer. If possible, it is best to perform this step in a fume hood.

Note: To accurately control timing of formaldehyde, the mixing is done using a heated magnetic stir plate and stir bar. Before the first use, check, using a thermometer, which temperature setting of the stir plate will keep the culture at $30^{\circ} \mathrm{C}$ during the mixing.

Critical: Never use formaldehyde that is $>3$ months old, preferably use formaldehyde < 1 month old. We recommend purchasing small bottles of formaldehyde and finishing them within a few (2-3) weeks after the first use, with minimal opening/closing of the bottle. Formaldehyde polymerizes when exposed to oxygen, which will compromise the cross-linking efficiency.

Critical: The timing and final concentration of formaldehyde used for crosslinking is crucial, since the extent of cross-linking strongly affects ChIP signals and downstream processing steps, see also (de Jonge et al, 2019). The optimal cross-linking parameters (concentration and timing) depend on the protein of interest. We recommend keeping the cross-linking time as short as possible, but cross-linking up to 30 minutes may be used, depending on the formaldehyde concentration.

4. Add Tris $4.5 \mathrm{M} \mathrm{pH} 8.0$ to a final concentration of $1.5 \mathrm{M}$ to the cultures by adding $52.9 \mathrm{ml}$ to stop the cross-linking reaction. Incubate the culture and Tris for $1 \mathrm{~min}$ using the same agitation as the cross-linking.

Note: For efficient quenching, use Tris pH 8.0 in a 2.25 fold molar excess. For $2 \%$ formaldehyde, use a final concentration of $1.5 \mathrm{M}$ Tris, and for $1 \%$ formaldehyde, use a final concentration of $750 \mathrm{mM}$ Tris.

Critical: To properly control the timing of cross-linking, the formaldehyde must be quenched efficiently. Many protocols use sub-stoichiometric levels (e.g. 125 $\mathrm{mM} / 250 \mathrm{mM}$ ) of glycine to quench. This is very inefficient, and cross-linking will continue in the presence of glycine, see also (de Jonge et al, 2019; Zaidi et al, 2017). Failure to efficiently quench formaldehyde can be a major source of variation in ChIP signals! Tris is a much more efficient quencher than the commonly used glycine (Hoffman et al, 2015; de Jonge et al, 2019; Sutherland et al, 2008). Although 
concerns have been raised that Tris might reverse the formaldehyde cross-links, we have found no evidence of this using short incubation times (de Jonge et al, 2019).

5. Split each culture over $3 \times 50 \mathrm{ml}$ tubes and spin at $3220 \mathrm{~g}(4000 \mathrm{rpm})$ for $3 \mathrm{~min}$ at $4^{\circ} \mathrm{C}$ (Eppendorf $\left.5810 \mathrm{R}\right)$.

Note: keep the samples cold in between the centrifugation steps, by putting them on ice. Try to be as quick as possible. Even though quenching with Tris is efficient and does not reverse cross-links, it is best to keep the time that samples spend on ice to a minimum.

6. Wash pellets by resuspending the first pellet in $10 \mathrm{ml}$ ice-cold TBS pH 7.5. Add the resuspended pellet to the second pellet and resuspend. Repeat this with the third pellet to combine the three pellets (final volume $10 \mathrm{ml}$ ) and spin down for $3 \mathrm{~min}$ at $3220 \mathrm{~g}(4000 \mathrm{rpm})$ at $4^{\circ} \mathrm{C}$ (Eppendorf $\left.5810 \mathrm{R}\right)$.

7. Remove supernatant, resuspend pellet in $1 \mathrm{ml}$ ice-cold $\mathrm{MQ}$, and transfer to a 2.0 $\mathrm{ml}$ Eppendorf tube.

8. Spin down at $3381 \mathrm{~g}(6000 \mathrm{rpm})$ for $20 \mathrm{sec}$ in a centrifuge (Eppendorf 5424).

9. Remove supernatant, freeze pellet in liquid nitrogen and store at $-80^{\circ} \mathrm{C}$.

Pause Point: The pellets can be stored at $-80^{\circ} \mathrm{C}$ for at least several months. 


\section{Bead beating procedure - Day 2}

Timing: $3-4$ hours

Cells are lysed and chromatin is fragmented. Fragmentation is needed to solubilize chromatin and to increase the resolution of ChIP.

Note: This protocol was optimized for $100 \mathrm{ml}$ of mid-log cells, OD $=0.8\left(2 \times 10^{7}\right.$ cells $/$ $\mathrm{ml})$. Preferably process 6 samples at once to increase speed and prevent proteolytic degradation of the samples. If need be, up to 12 samples can be processed at the same time. Be aware that with increasing number of samples (and thus processing time) the risk of proteolytic degradation of the protein of interest also increases.

Note: The FA lysis buffer is kept on ice and four different protease inhibitors are added. Approximately $1.9 \mathrm{ml}$ FA lysis buffer per sample is needed. Add the following protease inhibitors as listed in Table 1:

Critical: Keep the samples on ice all the time and pre-chill all tubes on ice! Work as fast as possible to prevent degradation.

Critical: Protein degradation during the procedure can have detrimental effects on the ChIP signal. It is therefore important that fresh protease inhibitors are added at the steps indicated in the protocol.

Table 1 Protease inhibitors to add to $1 \mathrm{ml}$ of FA lysis buffer.

\begin{tabular}{|l|l|l|}
\hline Name & Amount to add per $\mathbf{~ l l}$ & Note \\
\hline Aprotinin & $30 \mu \mathrm{l}$ & Stock at $4^{\circ} \mathrm{C} 33.3 \mathrm{x}$ \\
\hline Leupeptin & $1 \mu \mathrm{l}$ & Stock in $\mathrm{MQ}-20^{\circ} \mathrm{C}, 1000 \mathrm{x}$ \\
\hline Pepstatin A & $1 \mu \mathrm{l}$ & Stock in Methanol $-20^{\circ} \mathrm{C}, 1000 \mathrm{x}$ \\
\hline PMSF & $10 \mu \mathrm{l}$ & Stock in isopropanol $-20^{\circ} \mathrm{C}, 100 \mathrm{x}$ \\
\hline
\end{tabular}

10. Add $500 \mu \mathrm{l}$ of zirconium beads $(0.5 \mu \mathrm{m})$ to $2.0 \mathrm{ml}$ screw-cap tubes. Measure the right amount of beads using a $0.5 \mathrm{ml}$ Eppendorf tube. The zirconium beads will disrupt the cell wall upon bead beating in the genie disruptor.

Note: Pre-cool centrifuges for both $2.0 \mathrm{ml}$ and $50 \mathrm{ml}$ tubes (Eppendorf $5810 \mathrm{R}$ and 5424 R).

Alternatives: the cells may be lysed by making spheroplasts using zymolyase, which may be less cumbersome. However, zymolyase preparations are known to have proteases present, which may cause extensive proteolytic degradation of the protein of interest (de Jonge et al, 2019). If lysis with zymolyase is preferred, it would be best to add protease inhibitors to all wash and digestion buffers, and to wash extensively using $1 \mathrm{M}$ Sorbitol to remove the majority of proteases. However, caution is required to ensure that spheroplasts are not lysed during the washing steps. 
11. Add the protease inhibitors to the FA lysis buffer right before you add the buffer to the cells. For 6 cell pellets, add to $14.5 \mathrm{ml}$ of FA lysis buffer: $450 \mu \mathrm{l}$ aprotinin, $15 \mu \mathrm{l}$ pepstatin, $15 \mu \mathrm{l}$ leupeptin first and then $150 \mu \mathrm{l}$ PMSF.

Critical: PMSF is a highly toxic neurotoxin. Be very careful when preparing the stock, and always add this in a fume hood. To add, take the stock out of the $-20^{\circ} \mathrm{C}$ freezer and warm the tube by rubbing it between your hands (wearing gloves!). PMSF crystallizes when cold, it will need to be re-solubilized. Alternate between a gentle vortex and rubbing until re-solubilized. When you add the PMSF, mix immediately to prevent precipitation. Add PMSF last as it will lose activity rapidly in an aqueous environment.

12. Resuspend the frozen pellet carefully in $900 \mu \mathrm{l}$ of FA lysis buffer with protease inhibitors and transfer to a $2.0 \mathrm{ml}$ screw-cap tube with beads very slowly, to prevent any air getting trapped in the beads.

Optional: take a $5 \mu \mathrm{l}$ sample for western here (pre-bead beat sample). Add $5 \mu \mathrm{FA}$ lysis buffer, $2.5 \mu \mathrm{l}$ of $5 x$ sample buffer and boil for $30 \mathrm{~min} @ 95^{\circ} \mathrm{C}$.

Note: Try to prevent trapping air between the beads, as this will lead to foaming. If there is any air trapped in the beads: close tube and turn it upside down to release trapped air.

13. Add more FA lysis buffer containing protease inhibitors to fill the tube completely $(\sim 1 \mathrm{ml})$, keeping as little air as possible and close the tube carefully.

14. Disrupt cells with a genie disrupter at $4^{\circ} \mathrm{C}$ by bead beating 7 times $3 \mathrm{~min}$ (2850 rpm). Put samples on ice for 1 min between each run.

Note: During this time pre-chill the sonicator and prepare/label tubes needed for the next step!

15. Prepare $15 \mathrm{ml}$ tubes containing a $1 \mathrm{ml}$ pipette tip (cut the end of the $1 \mathrm{ml}$ tips to avoid blockage, Fig 1A).

16. Recover each extract by burning a hole in the bottom of the screw-cap tube with a hot $23 \mathrm{G}$ needle and quickly placing it in the $15 \mathrm{ml}$ tube on top of the $1 \mathrm{ml}$ pipette tip (Fig 1B). Burn a second hole in the top of the tube to facilitate release (Fig 1C). Centrifuge this combination at $201 \mathrm{~g}(1000 \mathrm{rpm})$ for 1 minute at $4^{\circ} \mathrm{C}$ (Eppendorf $5810 \mathrm{R}$ ).

17. Transfer the complete lysates to a $2.0 \mathrm{ml}$ Eppendorf tube and spin down at $1503 \mathrm{~g}$ (4000 rpm) for $2 \mathrm{~min}$ at $4^{\circ} \mathrm{C}$ (Eppendorf $\left.5424 \mathrm{R}\right)$.

Note: This step will remove the majority of the cell debris that could interfere in the subsequent sonication. The supernatant should contain the chromatin.

18. Transfer all supernatant to a new $2.0 \mathrm{ml}$ Eppendorf tube (take as much as possible $(\sim 1400 \mu \mathrm{l}))$ 

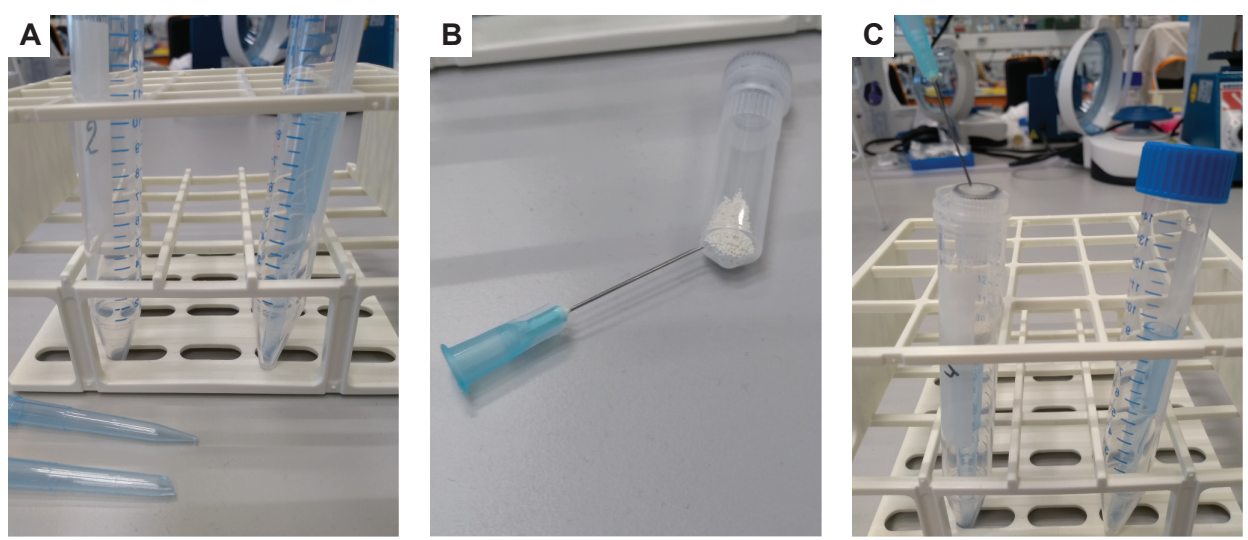

Figure 1 How to prepare 15-ml tubes for recovering chromatin.

(A) Cut the end of a $1 \mathrm{ml}$ pipette tip and place in a labelled $15 \mathrm{ml}$ tube. (B) Burn a hole in the bottom of the 2-ml screw cap tube containing the chromatin using a hot 23G needle and quickly place in the $15 \mathrm{ml}$ tube, on top of the $1 \mathrm{ml}$ pipette tip. (C) Burn a second hole in the top of the screw-cap tube, to facilitate the extraction. Although it is not shown in the figure, always keep all the tubes on ice.

Critical: With longer cross-linking times, there is an increased chance of chromatin co-precipitating with the pellet. If this happens, it should be visible as a vague white layer on top of the pellet. Make sure to transfer this chromatin pellet together with the supernatant, otherwise the majority of the (cross-linked) DNA will be lost. What counts as "long cross-linking times" depends on the concentration of formaldehyde used. With 1\% formaldehyde we noticed the co-precipitation of the chromatin when cross-linking for 20 or 30 minutes, but not with cross-linking for 10 minutes. We have not checked this using $2 \%$ formaldehyde, but extrapolating we expect that this could happen when cross-linking for $\mathbf{1 0}$ minutes or longer.

Optional: a second centrifugation step (21130g, 15000 rpm, 5424 R Eppendorf) may be added here to separate the chromatin from unbound / cytosolic proteins. This chromatin pellet should be resuspended in the same volume of FA lysis buffer (1400 $\mu \mathrm{l})$. The chromatin pellet is usually difficult to resuspend. A 23G-needle may be used to facilitate resuspending. We usually omit this step, but when the chromatin and soluble proteins are not separated, this means that unbound TF is mixed with the chromatin during all subsequent steps, which could potentially lead to in vitro binding to the DNA. When adding this step, bear in mind that with lower crosslinking times (5 min or less) the chromatin may not fully precipitate and a part of the chromatin may be lost. Keeping a part of the supernatant from this step for Western or DNA quantitation will show the efficiency of chromatin recovery.

19. Transfer $2 \times 300 \mu \mathrm{l}$ to $1.5 \mathrm{ml}$ Bioruptor Pico sonication tubes (300 $\mu \mathrm{l}$ per tube, this is about half of the total volume). Only half of the sample is used to make sure that the sonication and/or IP can be repeated if needed. Snap freeze the remainder of the cross-linked chromatin and store at $-80^{\circ} \mathrm{C}$.

20. Add fresh protease inhibitors. Make a mix (for 12 sonication tubes) of $63 \mu \mathrm{l}$ aprotinin, $2.1 \mu \mathrm{l}$ leupeptin and $2.1 \mu \mathrm{l}$ pepstatin. First add $4.8 \mu \mathrm{l}$ to all $300 \mu \mathrm{l}$ 
samples in sonication tubes and then add $3 \mu$ of PMSF (in fumehood), mix immediately by very gentle vortexing, making sure the extract does not touch the lid!

Optional: take a $10 \mu \mathrm{l}$ sample for western here (post lysis, pre sonication). Add 2.5 $\mu \mathrm{l}$ of $5 x$ sample buffer and boil for $30 \mathrm{~min} @ 95^{\circ} \mathrm{C}$.

21. Shear for 10 or 8 cycles $15^{\prime \prime}$ on, $30^{\prime \prime}$ off (Table 2 ) using a Bioruptor Pico that is connected to a water cooler, which is set to $4^{\circ} \mathrm{C}$.

Note: The parameters of sonication are highly dependent on the device used and need to be optimized for each device. The size range that should be aimed for is the same for all shearing devices (see note at step 32).

Note: During sonication the sample heats up due to the physical force that is exerted on the chromatin. Using short "on" times will keep the extent of heating to a minimum, which helps to prevent protein degradation. Large proteins (> 80-100 $\mathrm{kDa}$ ) are particularly sensitive to this degradation (de Jonge et al, 2019; Pchelintsev et al, 2016). If the degradation is very severe, enzymatic fragmentation with for example micrococcal nuclease (MNase) may be used instead of sonication.

Table 2 Suggested number of cycles $\left(15^{\prime \prime}\right.$ on, $30^{\prime \prime}$ off) to use for shearing the samples with a Bioruptor Pico, depending on the cross-linking time.

These are the suggested values for cross-linking with $2 \%$ formaldehyde.

\begin{tabular}{|l|l|}
\hline Time of cross-linking & Number of cycles \\
\hline $0 \mathrm{~min}$ & 10 \\
\hline $5 \mathrm{~min}$ & 10 \\
\hline $10 \mathrm{~min}$ & 8 \\
\hline $20 \mathrm{~min}$ & 8 \\
\hline
\end{tabular}

22. Spin down sample for $20 \mathrm{~min}$ at max speed $(21130 \mathrm{~g}, 15000 \mathrm{rpm}, 5424 \mathrm{R}$ Eppendorf) at $4^{\circ} \mathrm{C}$.

23. Combine the two supernatants per sample in a new $2.0 \mathrm{ml}$ Eppendorf tube.

24. Take $20 \mu \mathrm{l}$ for QC and take $1 \mu \mathrm{l}$ for Bradford analysis (dilute 1:41 by adding it to $40 \mu \mathrm{l}$ of MQ). Expect values around 2 - $5 \mu \mathrm{g} / \mu \mathrm{l}$.

Optional: also take $10 \mu \mathrm{l}$ for a Western (post sonication), add $2.5 \mu \mathrm{l}$ of $5 \mathrm{x}$ sample buffer and boil for $30 \mathrm{~min} @ 95^{\circ} \mathrm{C}$. (For the Western load $10 \mu \mathrm{l}$ on the gel). When running a Western, stain with an antibody that is suitable for Westerns.

25. Snap freeze the sample in liquid nitrogen and store at $-80^{\circ} \mathrm{C}$.

26. Continue with reverse cross-linking for QC.

Pause point: The chromatin can be stored at $-80^{\circ} \mathrm{C}$ for several months. 


\section{Reversing the cross-link for QC - Day 2 \& Day 3}

Timing: 4 hours (day 3)

A small portion of the sheared DNA (from bead beating procedure - step 26) is decross-linked, cleaned and analyzed using a Bioanalyzer to check the efficiency of the shearing.

27. Put together:

$20 \mu \mathrm{l}$ chromatin extract.

TE/SDS to $95 \mu 1$.

28. Reverse cross-link by incubating $12-16$ hours at $65^{\circ} \mathrm{C}$ in a thermoshaker $(800$ rpm).

Pause point: continue with cleaning of the DNA the next day

29. The next morning (Day 3): Add $5 \mu \mathrm{l}$ RNase A/T1 Mix and incubate for $30 \mathrm{~min}$ at $37^{\circ} \mathrm{C}$.

Note: A mix of RNAse A + RNAse T1 is used, because RNAse A treatment alone was often insufficient to digest all RNA, and a peak of small degraded RNA species was still visible in the Bioanalyzer electropherogram.

30. Add $40 \mu \mathrm{l}$ of proteinase $\mathrm{K}(10 \mu \mathrm{g} / \mu \mathrm{l})$ and incubate for 2 hours at $37^{\circ} \mathrm{C}$.

31. Clean up with the Qiagen PCR purification kit (all steps performed at $19-22^{\circ} \mathrm{C}$ ):

a. Add 5 volumes of PB buffer $(700 \mu \mathrm{l}$ for $140 \mu \mathrm{l}$ sample) and mix well.

b. Add $500 \mu \mathrm{l}$ sample to spin column.

c. Spin for $1 \mathrm{~min}$ at max speed (21130g, $15000 \mathrm{rpm}, 5424 \mathrm{R}$ Eppendorf).

d. Discard flow-through and add the remaining sample $(340 \mu \mathrm{l})$.

e. Spin for $1 \mathrm{~min}$ at max speed, discard flow-through.

f. Wash 3 times with $500 \mu \mathrm{l}$ of PE and spin down 1 min at max speed, discard flow-through between washes.

g. Transfer column to new $2.0 \mathrm{ml}$ tube without lid and spin down at max speed for $1 \mathrm{~min}$ (to remove last bit of PE buffer).

h. Elute by adding $40 \mu \mathrm{l}$ of EB, incubate for $1 \mathrm{~min}$, and spin down at max speed in $1.5 \mathrm{ml}$ Eppendorf tube.

Note: A Qiagen PCR cleanup kit is used to clean up QCs, as well as the IP and input samples. The recovery of this kit is about 70\%. We tried several other cleanups (Zymoclear ChIP kit, Ampure beads, phenol chloroform isolation and Qiagen MinElute DNA kit), but the Qiagen PCR cleanup kit was equal or better in both recovery and reproducibility. If preferred, any alternative cleanup strategy may be used as well, as long as it provides consistent recoveries.

32. Check DNA fragment size by loading $1 \mu \mathrm{l}$ of purified DNA on Bioanalyzer with a High-Sensitivity DNA Chip (or equivalent).

Note: The electropherogram of the Bioanalyzer should show a peak between 200-300 


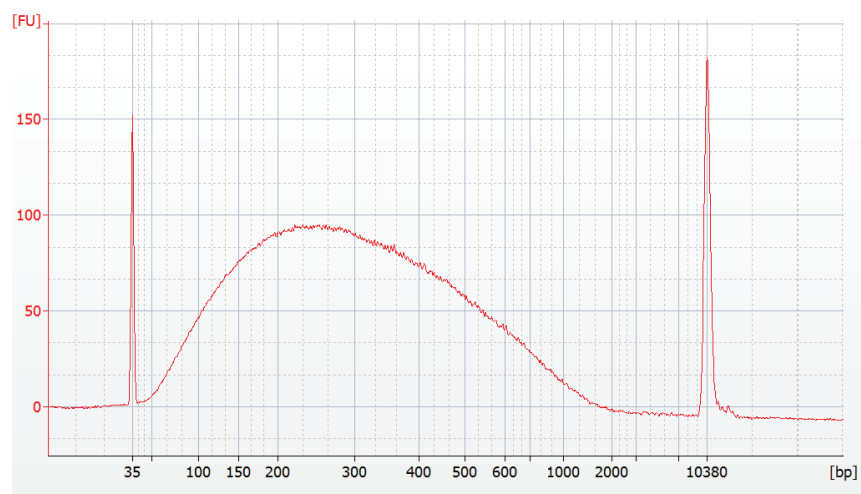

Figure 2 Electropherogram showing the distribution of DNA fragments of a properly sheared sample.

bp, as this fragment sizeis optimal for sequencing(Fig2). Depending on the application, longer fragment sizes may also be acceptable. If there is still a large amount of longer fragments detected, shearing of the chromatin can be repeated for a few additional cycles, but care should be taken that this can also increase proteolytic degradation of these samples. When shearing is repeated, fresh protease inhibitors should be added.

Pause point: The chromatin can be stored at $-80^{\circ} \mathrm{C}$ for several months. 


\section{Immunoprecipitation using magnetic beads - Day 3}

Timing: $4-5$ hours

The protein of interest is recognized by an antibody that binds to magnetic beads. Non-specific binding is largely washed away with different wash buffers. First bind the antibody to chromatin (steps 33-37). While incubating, prepare the magnetic beads (steps 38-47). Next, bind chromatin bound antibody to the beads (step 48). Finally, wash the beads (steps 49-52) before proceeding with reversal of cross-linking and cleanup (steps 53-62).

33. Thaw the chromatin on ice and take a $20 \mu \mathrm{l}$ aliquot for input control. Keep on ice while processing the IP samples. Continue with the input samples at step 58.

Note: During this step make a mix of (per 6 samples): $210 \mu$ l Aprotinin, $7 \mu 1$ Leupeptin and $7 \mu l$ Pepstatin.

34. Transfer $450 \mu \mathrm{l}$ of the chromatin to a (cold) $1.5 \mathrm{ml}$ Eppendorf tube.

35. Add $16 \mu \mathrm{l}$ of the protease inhibitor mix and subsequently add $5 \mu$ PMSF (in fumehood). Mix immediately by vortexing gently.

36. Add $1.2 \mu \mathrm{g}(1 \mu \mathrm{l})$ of antibody to the chromatin extract.

Note: The amount of antibody that is needed is dependent on the antibody and the abundance of the target. The optimal amount of antibody can best be determined empirically. $1.2 \mu \mathrm{g}$ antibody worked well for all V5-tagged proteins that we tested.

37. Incubate with rotation $(20 \mathrm{rpm})$ for $2 \mathrm{~h}$ at $4{ }^{\circ} \mathrm{C}$.

Note: The optimal incubation time depends on the antibody used; this can best be determined empirically.

38. During the incubation of the chromatin with the antibody, the magnetic beads can be prepared: Resuspend the magnetic beads (Dynabeads) in the vial (vortex $>30 \mathrm{sec}$ ).

39. Transfer $25 \mu \mathrm{l}(0.75 \mathrm{mg})$ beads per ChIP to a $1.5 \mathrm{ml}$ Eppendorf tube.

Note: when doing many ChIPs, close the bottle and vortex again for a few seconds after filling a few samples to keep the beads well mixed.

40. Place the tube on the magnet (DynaMag-2) to separate the beads from the solution and remove the supernatant.

41. Wash once with $500 \mu \mathrm{l}$ PBS-T (PBS/0.02\% Tween-20) by adding the PBS-T followed by gentle vortexing.

42. Place the tube on the magnet to separate the beads from the solution and remove the supernatant.

43. Add $400 \mu \mathrm{l}$ PBS $+25 \mu \mathrm{l}$ BSA $(10 \mathrm{mg} / \mathrm{ml}$ in TBS-T).

44. Incubate with rotation $(20 \mathrm{rpm})$ for $\sim 2 \mathrm{~h}$ at $4{ }^{\circ} \mathrm{C}$. 
45. About 5-10 minutes before the incubation of the chromatin + antibody is ready. Place the tube on the magnet to separate the beads from the solution and remove the supernatant

46. Wash once with $500 \mu \mathrm{l}$ PBS-T.

47. Place the tube on the magnet to separate the beads from the solution and remove the supernatant. Close the tube to prevent the beads from drying up.

48. After 2 hours of incubation at $4^{\circ} \mathrm{C}$ add $5 \mu \mathrm{l}$ PMSF to the sample containing the antigen (CE $450 \mu \mathrm{l}+\mathrm{V} 5$ antibody), add to the beads and gently vortex to mix the beads with the chromatin. Incubate with rotation $(20 \mathrm{rpm})$ for $20 \mathrm{~min}$ at $19-22^{\circ} \mathrm{C}$ to allow the antibody to bind to the beads.

Note: Depending on the antibody this incubation may need to be longer.

49. Spin down briefly and place the tube on the magnet.

Optional: Transfer the supernatant to a clean tube for further analysis, if desired. This can be used, for example, to examine the fraction of protein that was not immunoprecipitated.

50. Wash the bead complex 2 times using $200 \mu \mathrm{l}$ PBS for each wash. Separate on the magnet between each wash, remove supernatant and resuspend by gentle vortexing.

Optional: if the protein of interest is particularly sensitive to proteolytic degradation add the protease inhibitors also to all the ChIP wash buffers. Per $1 \mathrm{ml}$ of wash buffer add: $30 \mu \mathrm{l}$ aprotinin, $1 \mu \mathrm{l}$ pepstatin, $1 \mu \mathrm{l}$ leupeptin and $10 \mu \mathrm{l}$ PMSF.

Optional: If the protein of interest is a particularly strong binder, more stringent washes may help to remove non-cross-linked proteins. Use wash buffer 1 and/or wash buffer 2 (2-3 washes of $200 \mu \mathrm{l}$ each). Taking along a non-cross-linking control should show whether a protein can bind to DNA without cross-linking and whether the more stringent washes are sufficient. More stringent washes will significantly reduce the background signal, but depending on the affinity of the antibody may also reduce the specific ChIP signal.

51. Wash the beads 1 time using $200 \mu$ PBS-T. Resuspend by gentle vortexing, separate on the magnet after washing and remove supernatant.

52. Resuspend the beads in $100 \mu \mathrm{l}$ PBS-T and transfer the bead suspension to a clean LoBind Eppendorf tube. This is recommended to avoid co-elution of proteins bound to the tube wall.

53. Proceed to Elution and reverse cross-linking. 


\section{Elution and reverse cross-linking - Day 3 \& Day 4}

Timing: 4 hours

The protein-DNA cross-links are reversed by heating and the DNA is eluted from the beads.

54. Place the tube (from step 52 in "Immunoprecipitation using magnetic beads") on the magnet and remove the supernatant.

55. Add $98 \mu \mathrm{l}$ TE/SDS.

56. Resuspend the bead-antibody-antigen complex by gentle vortexing.

57. Reverse the cross-links for $12-16$ hours using shaking $(800 \mathrm{rpm})$ at $65^{\circ} \mathrm{C}$.

58. Forinputsamples, add $75 \mu \mathrm{TE} / \mathrm{SDS}$ to the $20 \mu$ lextract(from Immunoprecipitation using magnetic beads - step 33), mix and incubate using shaking (800 rpm) at $65^{\circ} \mathrm{C}$ as well.

Pause point: continue with cleaning of the DNA the next day

59. The next morning (Day 4): add $2 \mu \mathrm{l}$ RNAse A/T1 mix to the IP samples and $5 \mu \mathrm{l}$ to the input samples and incubate $30 \mathrm{~min} @ 37^{\circ} \mathrm{C}$.

60. Add $40 \mu \mathrm{l}$ of proteinase $\mathrm{K}\left(10 \mathrm{mg} / \mathrm{ml}\right.$ in TE), and incubate for 2 hours at $37^{\circ} \mathrm{C}$.

61. Place the tube on the magnet and transfer the supernatant containing eluted DNA (IPs) to a clean LoBind-tube.

62. Clean up with the Qiagen PCR purification up kit, similar to the cleanup for QC.

a. Add 5 volumes of PB buffer (700 $\mu$ l for $140 \mu$ l sample) and mix well

b. Add $500 \mu \mathrm{l}$ sample to spin column.

c. Spin for $1 \mathrm{~min}$ at max speed (21130g, $15000 \mathrm{rpm}, 5424 \mathrm{R}$ Eppendorf).

d. Discard flow-through and add the remaining sample $(340 \mu \mathrm{l})$.

e. Spin for 1 min at max speed, discard flow-through.

f. Wash 3 times with $500 \mu \mathrm{l}$ of PE and spin down at max speed, discard flowthrough between washes.

g. Transfer column to an empty $2.0 \mathrm{ml}$ tube without lid and spin down at max speed for $1 \mathrm{~min}$ to remove last bit of PE buffer.

h. Elute by adding $40 \mu \mathrm{l}$ of EB, incubate for $>1 \mathrm{~min}$, and spin down at max speed in a new $1.5 \mathrm{ml}$ Lobind tube.

Optional: The DNA can also be eluted in $30 \mu \mathrm{l}$ of EB for more concentrated DNA.

63. Quantify the binding using qPCRs and/or proceed to make sequencing libraries.

Pause point: the DNA can be stored at $4^{\circ} \mathrm{C}$ for several days. For long term storage we recommend storing the cleaned DNA at $-20^{\circ} \mathrm{C}$. 


\section{Quantification of binding using qPCR - Day 5}

Timing: 4 hours

Binding levels of the protein of interest are quantified using qPCR. The relative binding frequency is estimated by calculating the percentage of input recovered in the IP.

Note: If it is already known which loci should be bound by the protein of interest, binding to a few of these regions can be measured using qPCR. Even if the aim is to quantify the samples using sequencing, an initial check by qPCR is recommended to make sure that the procedure worked.

Note: This qPCR protocol was designed for $10 \mu$ reaction in 384-well format using IQ SYBR Green super mix (Bio-Rad) and a CFX384 Touch Real-Time PCR Detection system (Bio-Rad). The exact procedure differs depending on the reagents and machine used, but the general design principles are the same for all qPCRs. An overview of the reagents needed for 1 qPCR reaction are listed in Table 3.

Table 3 Reagents of a single $10 \mu \mathrm{qPCR}$ reaction.

\begin{tabular}{|l|l|}
\hline Reagents & Volume \\
\hline IQ SYBR Green super mix & $5 \mu \mathrm{l}$ \\
\hline MQ & $2.8 \mu \mathrm{l}$ \\
\hline Forward + Reverse primer mix $(10 \mu \mathrm{M}$ each $)$ & $0.2 \mu \mathrm{l}$ \\
\hline Template & $2 \mu \mathrm{l}$ \\
\hline Optional: Precision blue & $0.014 \mu \mathrm{l}(1 / 70 \mu \mathrm{l})$ \\
\hline
\end{tabular}

Critical: When designing primers for $\mathrm{qPCR}$, make sure the amplicons are not too big (preferably $<140 \mathrm{bp}$, optimally $\sim 100 \mathrm{bp}$ ). If the average size of the sheared DNA is close to the size of the amplicon, this will lead to inefficient amplification.

64. Make a working stock of each primer pair combination by adding both the forward and the reverse primer to a final concentration of $10 \mu \mathrm{M}$. (For example, add to $160 \mu \mathrm{MQ}, 20 \mu \mathrm{l}$ of $100 \mu \mathrm{M}$ forward primer and $20 \mu \mathrm{l}$ of $100 \mu \mathrm{M}$ reverse primer for a $200 \mu \mathrm{l}$ stock).

65. Prepare a 96-well plate with the DNA as is shown in Fig 3. First fill the plate with MQ needed in each well, then add the DNA.

a. Make a calibration curve of sheared reference DNA in the first column of the plate. Make a 5-step 10-fold serial dilution of the reference DNA. Also fill 2 wells with MQ as a no template control. Measure at least in duplicate, in which case $4 \mu \mathrm{l}$ per primer pair is needed.

b. In the next column(s) add the IPs. Depending on the efficiency of the ChIP, and the abundance of the protein of interest, the IPs can be diluted 2x / $4 x$. Always measure the IPs in technical quadruplicate, $8 \mu$ l per IP per primer pair is needed.

c. Add the diluted inputs (50x diluted) in the column(s) after the IPs. If 


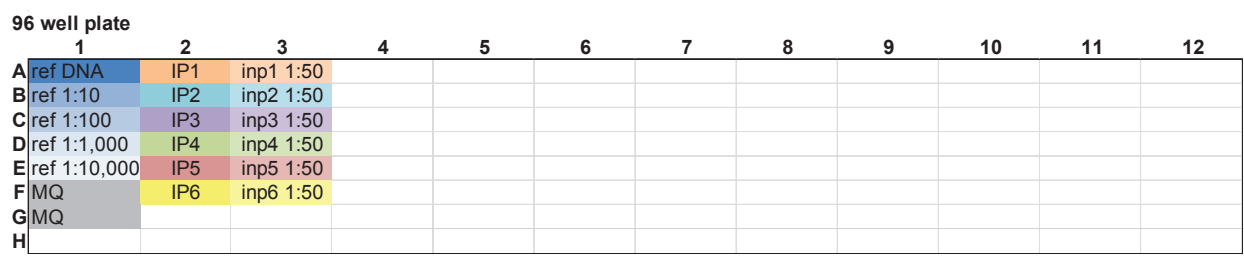

Figure 3 Example of the layout for the 96-well plate containing the (un)diluted standard curve, IP and input samples.

This example is for 6 IPs.

possible, always measure the inputs in technical quadruplicate as well. If more space is needed on the 384-well plate, the inputs can be measured in triplicate as well.

Alternatives: When only a few samples are run at the same time, PCR strip tubes may also be used.

Note: When doing qPCR, always take a standard curve along for each primer pair on each plate. It is best if this standard is created from fragmented, cleaned, genomic DNA and that the same material is used for all standard curves. This way different plates can be compared with each other. Ideally, this reference DNA should be isolated from the same strain or background as the samples of interest. Our undiluted reference DNA has a concentration of $\sim 0.25 \mathrm{ng} / \mu \mathrm{l}$.

Critical: Make sure to properly mix after each dilution of the standard curve and the samples. Failure to mix properly will give unreproducible qPCR results.

66. Make a pre-mix of $2.8 \mu \mathrm{lMQ}$ and $5 \mu \mathrm{l}$ IQ SYBR Green super mix for the number of desired reactions $+10 \%$. Mix well and pipette $7.8 \mu \mathrm{l}$ x number of desired reactions per primer pair $+5 \%$ to labelled $2.0 \mathrm{ml}$ tubes.

67. Add $0.2 \mu$ forward + reverse primers mix $x$ the number of reaction per primer pair $+5 \%$ to the tubes to make the primer-pair specific master mixes. Mix well.

Optional: When using BR white plates (Bio-Rad) it can be tricky to see which wells are filled and which are not. We usually add precision blue in a 1:700 dilution to each master mix to more easily visualize the filled wells.

68. Add $8 \mu \mathrm{l}$ of the primer specific master mixes to wells of the 384-well plate. It is recommended to use an electronic pipette to increase speed and accuracy.

Note: Keep both plates cold during preparation, to keep evaporation to a minimum. If possible, use a plate cooler for the 384-well plate or an inverted heat block in a bucket filled with ice, to provide a flat surface.

69. Using an 8-channel multichannel pipette transfer column-by-column $2 \mu \mathrm{l}$ of template from the 96-well plate to the 384-well plate (Fig 4). 


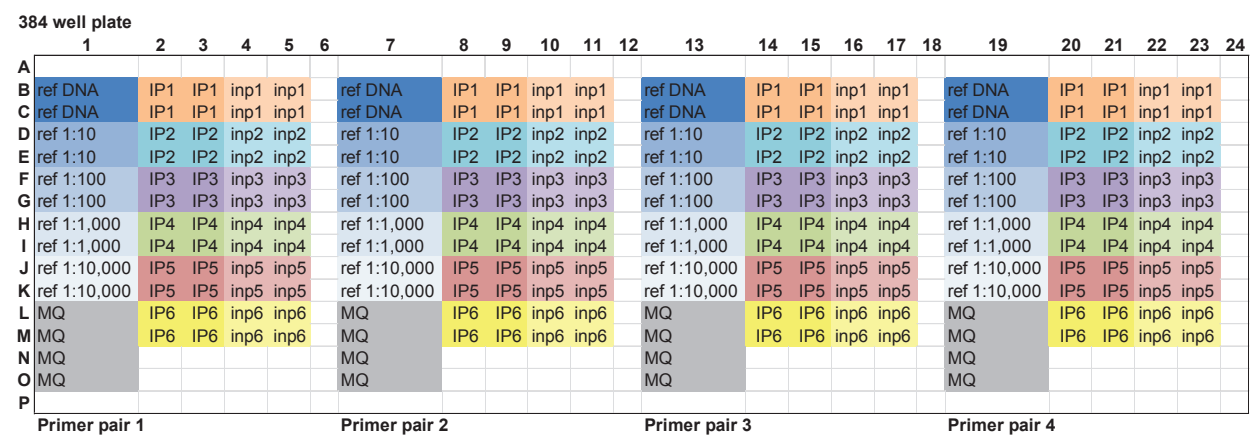

Figure 4 Example of a 384-well plate layout for 6 IPs and 4 primer pairs.

Note: To keep track of which wells are filled and which are not, we recommend printing out the plate layout and mark where you are after each template addition.

70. When all the DNA is transferred seal the plate properly with a seal compatible with qPCR.

Note: Check the seal for damage or dirty spots. The qPCR machine will measure the fluorescence through the seal, anything on the seal that changes the path of the light can affect the measured values.

Note: To mix, briefly vortex the plate at half speed using a standard vortex followed by a quick spin (200g).

71. Run the qPCR using an appropriate program. An example of a quick 2-step protocol can be found in Table 4.

Table 4 Example of a 2-step qPCR program with melting curve

\begin{tabular}{|l|l|l|}
\hline Step & Temperature & Time \\
\hline 1 & $95^{\circ} \mathrm{C}$ & $3: 00$ \\
\hline 2 & $95^{\circ} \mathrm{C}$ & $0: 15$ \\
\hline 3 & $60^{\circ} \mathrm{C}$ & $0: 30+$ measure fluorescence \\
\hline 4 & Go to step $2,39 \times$ more times & $1: 00$ \\
\hline 5 & $95^{\circ} \mathrm{C}$ & \multicolumn{2}{|l|}{} \\
\hline 6 & Melting curve: $70^{\circ} \mathrm{C}$ to $95^{\circ} \mathrm{C}$, increment $0.5^{\circ} \mathrm{C}+$ measure fluorescence \\
\hline
\end{tabular}

Note: A 2-step protocol can only be used with primers that have a melting temperature $\geq 61^{\circ} \mathrm{C}$ in the conditions of the qPCR. If possible, take this into account when designing primers. The conditions of the qPCR using IQ SYBR Green super mix are: $\mathrm{Na}^{+} 50 \mathrm{mM}$, Oligos $0.2 \mu \mathrm{M}, \mathrm{Mg}^{++} 6 \mathrm{mM}$ and $\mathrm{dNTP} 0.4 \mathrm{mM}$

Note: A melting curve at the end of the program is important to confirm that only a 
single specific product was formed.

72. Calculate the efficiency of the primers (the Bio-Rad CFX Meastro software will do this automatically). The efficiency should be between $90 \%-110 \%$ (1.9-2.1).

73. After exporting the data to the format of choice (for example excel file), calculate the starting quantities (SQs) of all reactions for each primer pair using the corresponding standard curve (the Bio-Rad CFX Meastro software will do this automatically).

74. First calculate the average SQ of each sample by averaging the four replicates.

Note: sometimes a reaction of a single replicate can fail. If there is a clear single outlier, this outlier can be removed to get a more accurate estimate of the SQ. As a rule of thumb, the replicates should have a coefficient of variation (standard deviation / average) $<20 \%$.

75. Correct each reaction for the dilution by multiplying the SQs by the dilution factor.

76. Correct all samples for the starting volume: $20 \mu \mathrm{l}$ for the inputs and $450 \mu \mathrm{l}$ for the IPs.

77. Calculate the $\%$ of input value by dividing the value of each IP by its corresponding input. See supplemental table for an example of these calculations.

Note: For accurate quantitation, the $\mathrm{Cq}(\mathrm{Ct})$ values of both the IP and the input samples should be within the values of the standard curve.

Note: Always include a background control with each qPCR: a promoter that does not have binding of the TF of interest. When a background control is included, the ratio over background can be calculated. However, if the background is very low, which is usually the case when using magnetic beads, the background can be hard to quantify using qPCR. If there is a lot of variation in the background signal, the enrichment over background will also have a lot of variation. 


\section{Expected outcomes}

A successful ChIP should show a clear enrichment of its expected binding sites over background. The actual values of the ChIP signal (\% of input) varies between proteins. This is dependent on the frequency of binding, or, in other words, the percentage of cells in the population where the protein of interest is bound at a given time, as well as the efficiency of the IP itself. For strongly bound histone proteins, which are relatively easy to ChIP, enrichment values of $\sim 20 \%-50 \%$ can be reached. Abundant transcription factors often have ChIP signals between $0.5 \%$ - 5\% of input, while for less abundant proteins values of $0.1 \%$ of input or lower can be expected.

An example of binding levels of an abundant transcription factor, Abf1, to two of its targets (FCF1 and NHX1) and a background region (TFC1) are shown in Fig 5. A wildtype (WT) strain, without a V5 tagged protein, is shown as a control. Indeed, the WT IP shows no binding or enrichment of any of the targets over background (Fig 5A and 5B). The target FCF1 has stronger binding compared to the target NHX1. This difference in binding levels is also observed when quantifying the binding using ChIP-seq (Fig 5C). An example of how these values were calculated can be found in the supplemental table.

\section{Limitations}

Although ChIP is a very common technique to assess protein-DNA binding, it has clear limitations. Formaldehyde can only form cross-links between DNA and proteins when they are $\sim 2 \AA$ apart (Hoffman et al, 2015). This means that proteins that do not directly contact DNA can only be cross-linked to DNA through other proteins, which is inefficient. Use of a protein-protein cross-linker such as for example dimethyl adipimidate or disuccinimidyl glutarate together with formaldehyde leads to more efficient ChIP for these proteins (Kurdistani \& Grunstein, 2003; Tian et al, 2012). For a complete list of protein cross-linkers and their properties see (Kurdistani \& Grunstein, 2003; Tian et al, 2012). In addition, formaldehyde mainly forms cross-links between $\mathrm{G} / \mathrm{C}$ bases and specific residues in the protein of interest. If there are limited positions where these are in close proximity, proteins may be less efficiently cross-linked to some sites compared to others (Rossi et al, 2018a). There is evidence that, at least for one protein, interactions with DNA that are very short lived ( $<5 \mathrm{~s}$ ) are not efficiently detected by ChIP(Schmiedeberg et al, 2009). This suggests that ChIP might not be suitable for proteins that bind DNA only very transiently.

Most ChIP protocols use sonication to fragment DNA. If such a protocol is used for ChIP sequencing, the resolution of the ChIP peaks is limited by the average fragment size. If a higher resolution is required, the DNA may be further fragmented following sonication, using $\lambda$-exonuclease (Rhee \& Pugh, 2011; Rossi et al, 2018b) or the DNA may be fragmented enzymatically using MNase instead of sonication (Gutin et al, 2018; Skene \& Henikoff, 2015). The enzymatic fragmentation will create smaller fragments that increase the resolution of 
A

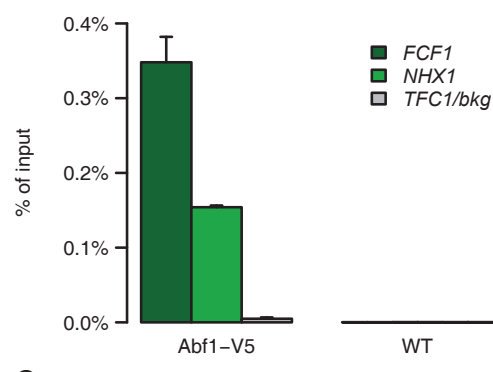

C

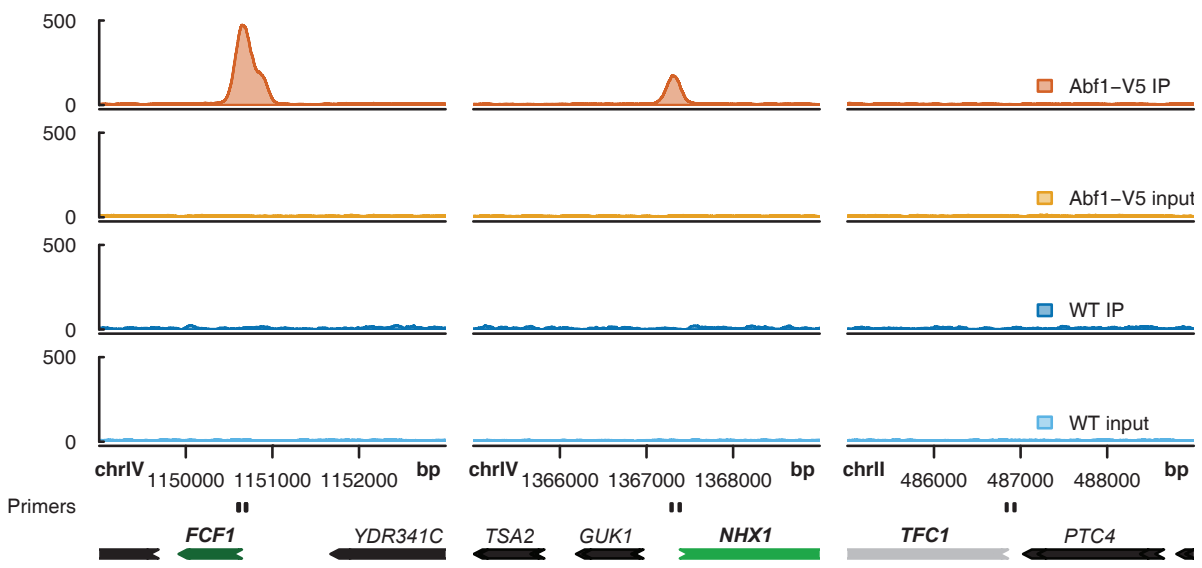

B

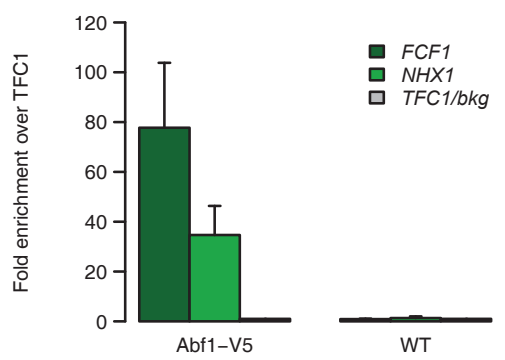

Figure 5 Binding levels of the yeast protein Abf1 quantified using ChIP.

(A and B) barplots showing the results from qPCR represented as (A) \% of input or (B) fold enrichment over a background region (TFC1). A wild-type (WT) strain without the V5 tag shows no binding or enrichment of the Abf1 targets. Error bars represent the standard deviation of 3 independent biological replicates. (C) Genome browser snapshot showing the binding, or lack thereof, of Abf1 (top lane) to the same targets as the qPCR. The wild-type strain shows no binding. All regions are scaled to the same height. The black bars represent the location of the primers used for the respective qPCRs. All tracks show the average signal of three biological replicates. The data that was used to generate (A) and (B) is provided in the supplemental table, which is available online.

the sequencing but will make it more difficult to quantify binding by qPCR. ChIP is known to have artefacts. Genomic loci with high expression (such as tRNA or highly expressed genes) often show up as peaks in ChIP-seq or ChIPchip experiments (Park et al, 2013; Teytelman et al, 2013). Long cross-linking times increase the severity of these artefacts (Baranello et al, 2015). If there is interest to quantify protein binding to such loci, proper controls should be taken along, such as ChIP for a protein that does not bind DNA (e.g. GFP). 


\section{Troubleshooting}

\section{Problem}

Low DNA yield (visible in the Bioanalyzer electropherogram, step 32)

\section{Potential Solution}

Low DNA yields can be caused by inefficient cell lysis. The extent of lysis can be assessed by taking a small aliquot of cell lysate and examine this using a hemocytometer under a standard microscope (after step 14). Longer cross-linking times may make the cell wall tougher and this can reduce lysis efficiency.

With long cross-linking times, the chromatin may co-precipitate with the unbroken cells and cell debris (Bead beating procedure, step 17). If this happens, the chromatin is visible as a vague white layer on top of the pellet. Make sure to take as much of this chromatin layer as possible together with the rest of the supernatant. Although we did not test this, centrifugation at a lower speed could prevent the chromatin from co-precipitating with the cell debris.

\section{Problem}

Inconsistent shearing of DNA (visible in the Bioanalyzer electropherogram, step 32)

\section{Potential Solution}

When shearing the chromatin (step 21), it is important to closely follow the recommendations of the manufacturer of the sonicator. For example, the Bioruptor Pico has a maximum limit of buffer used for each of its tubes. When the volume in the tubes exceeds limit, the shearing can become unreproducible. We also noticed that using lower concentrations of chromatin (by diluting the samples) can help achieve more consistent shearing. In this case, also bigger volumes should be used in the IP step.

Potentially, a higher concentration of SDS could help to increase the reproducibility of the shearing. Higher SDS concentrations can, however, interfere with the subsequent IP step. The samples may need to be diluted with FA lysis buffer without SDS to lower the concentration again.

As an alternative for sonication, enzymatic fragmentation using for example MNase could also be used. When using MNase, thelysis buffer may need to be adapted to allow efficient MNase digestion. We have not tested the lysis efficiency and compatibility with MNase digestion of different lysis buffers. Alternatively, after separating the chromatin from the cell debris, the chromatin may be precipitated using a highspeed spin (optional centrifugation after step 18). The pelleted chromatin can then be resuspended using an MNase digestion buffer. The latter strategy is preferred as this circumvents the need to adapt the lysis buffer. Be aware that extensive MNase 
digestion will create smaller fragments compared to sonication. This will increase the resolution of ChIP-seq, but will make quantification with qPCR more difficult.

\section{Problem}

\section{Low ChIP signal}

\section{Potential Solution}

Formaldehyde polymerizes in the presence of oxygen. When the formaldehyde is old, or the bottle is opened very frequently, formaldehyde may polymerize extensively. This will lower the cross-linking efficiency. Replace the bottle of formaldehyde when this happens.

With very stringent washes (steps 50-52), the binding of the antibody to the protein of interest may be disrupted, depending on the affinity. Less stringent washes may be used, but care has to be taken that the protein of interest does not bind to DNA in vitro during the procedure. Bear in mind that washing less stringently will also increase the background levels.

The protein of interest may degrade extensively during the procedure, which happens for large proteins, especially. Take aliquots at several steps of the protocol (indicated as optional steps at step 12, 20 and 24) and determine the extent of degradation at each step using Western blotting. If extensive degradation is a problem, consider adding more protease inhibitors, sonicating more gently or enzymatic fragmentation of the DNA (see problem: inconsistent shearing of DNA).

\section{Problem}

High variability in ChIP signal

\section{Potential Solution}

ChIP protocols involve many steps, and small differences at any step can lead to changes in ChIP signal. It is therefore important to be consistent and accurate at all steps. The main sources of variation are often differences in the extent of cross-linking, protein degradation or DNA shearing. It is therefore important to accurately control the time of cross-linking, to make sure that the samples remain cool throughout the procedure, to pre-cool all tubes and buffers and to add protease inhibitors at the steps indicated.

When the background signal is very low, there may only be a few molecules present in the qPCR reaction. At this lower limit, a larger number of replicates is needed to accurately estimate the background levels. Although the limit may depend on the experimental setup, as a rule of thumb Cq values above 30 will be more variable. Values close to 35 likely represent only a few molecules present in the reaction, and 
therefore will be hard to accurately quantify.

\section{Author contributions}

Conceptualization, W.d.J., P.K and F.H.; Investigation, W.d.J. and M.B.; Writing Original Draft, W.d.J. and F.H; Writing - Review \& Editing, W.d.J., M.B., P.K and F.H.; Funding Acquisition, P.K and F.H.; Supervision, P.K and F.H.

\section{Acknowledgements}

We would like to thank Marit de Kort for testing and critical reading of the protocol. This work was supported by the Netherlands Organisation for Scientific Research (NWO) grant 86411010 and by the European Research Council (ERC) grant 671174 DynaMech.

\section{References}

Aparicio O, Geisberg JV, Struhl K (2004) Chromatin immunoprecipitation for determining the association of proteins with specific genomic sequences in vivo. Curr Protoc Cell Biol Chapter 17: Unit 17.7

Baranello L, Kouzine F, Sanford S, Levens D (2015) ChIP bias as a function of cross-linking time. Chromosome Res 24: 175-181

Gutin J, Sadeh R, Bodenheimer N, Joseph-Strauss D, Klein-Brill A, Alajem A, Ram O, Friedman N (2018) Fine-Resolution Mapping of TF Binding and Chromatin Interactions. Cell Rep 22: 2797-2807

Hoffman EA, Frey BL, Smith LM, Auble DT (2015) Formaldehyde Crosslinking: A Tool for the Study of Chromatin Complexes. J Biol Chem 290: 26404-26411

de Jonge WJ, Brok M, Kemmeren P, Holstege FCP (2019) An extensively optimized chromatin immunoprecipitation protocol for quantitatively comparable and robust results. bioRxiv: 835926

Kurdistani SK, Grunstein M (2003) In vivo protein-protein and protein-DNA crosslinking for genomewide binding microarray. Methods 31: 90-95

Park D, Lee Y, Bhupindersingh G, Iyer VR (2013) Widespread misinterpretable ChIP-seq bias in yeast. PloS One 8: e83506

Pchelintsev NA, Adams PD, Nelson DM (2016) Critical Parameters for Efficient Sonication and Improved Chromatin Immunoprecipitation of High Molecular Weight Proteins. PloS One 11: $\mathrm{e} 0148023$

Rhee HS, Pugh BF (2011) Comprehensive genome-wide protein-DNA interactions detected at single-nucleotide resolution. Cell 147: 1408-1419

Rossi MJ, Lai WKM, Pugh BF (2018a) Genome-wide determinants of sequence-specific DNA binding of general regulatory factors. Genome Res 28: 497-508

Rossi MJ, Lai WKM, Pugh BF (2018b) Simplified ChIP-exo assays. Nat Comm 9: 1-13

Schmiedeberg L, Skene P, Deaton A, Bird A (2009) A Temporal Threshold for Formaldehyde Crosslinking and Fixation. PLOS ONE 4: e4636

Skene PJ, Henikoff S (2015) A simple method for generating high-resolution maps of genomewide protein binding. eLife 4: e09225 
Sutherland BW, Toews J, Kast J (2008) Utility of formaldehyde cross-linking and mass spectrometry in the study of protein-protein interactions. J Mass Spectrom JMS 43: 699-715

Teytelman L, Thurtle DM, Rine J, van Oudenaarden A (2013) Highly expressed loci are vulnerable to misleading ChIP localization of multiple unrelated proteins. Proc Natl Acad Sci U S A 110: 18602-18607

Tian B, Yang J, Brasier AR (2012) Two-step Crosslinking for Analysis of Protein-Chromatin Interactions. Methods Mol Biol Clifton NJ 809: 105-120

Zaidi H, Hoffman EA, Shetty SJ, Bekiranov S, Auble DT (2017) Second-generation method for analysis of chromatin binding with formaldehyde-cross-linking kinetics. J Biol Chem 292: 19338-19355 

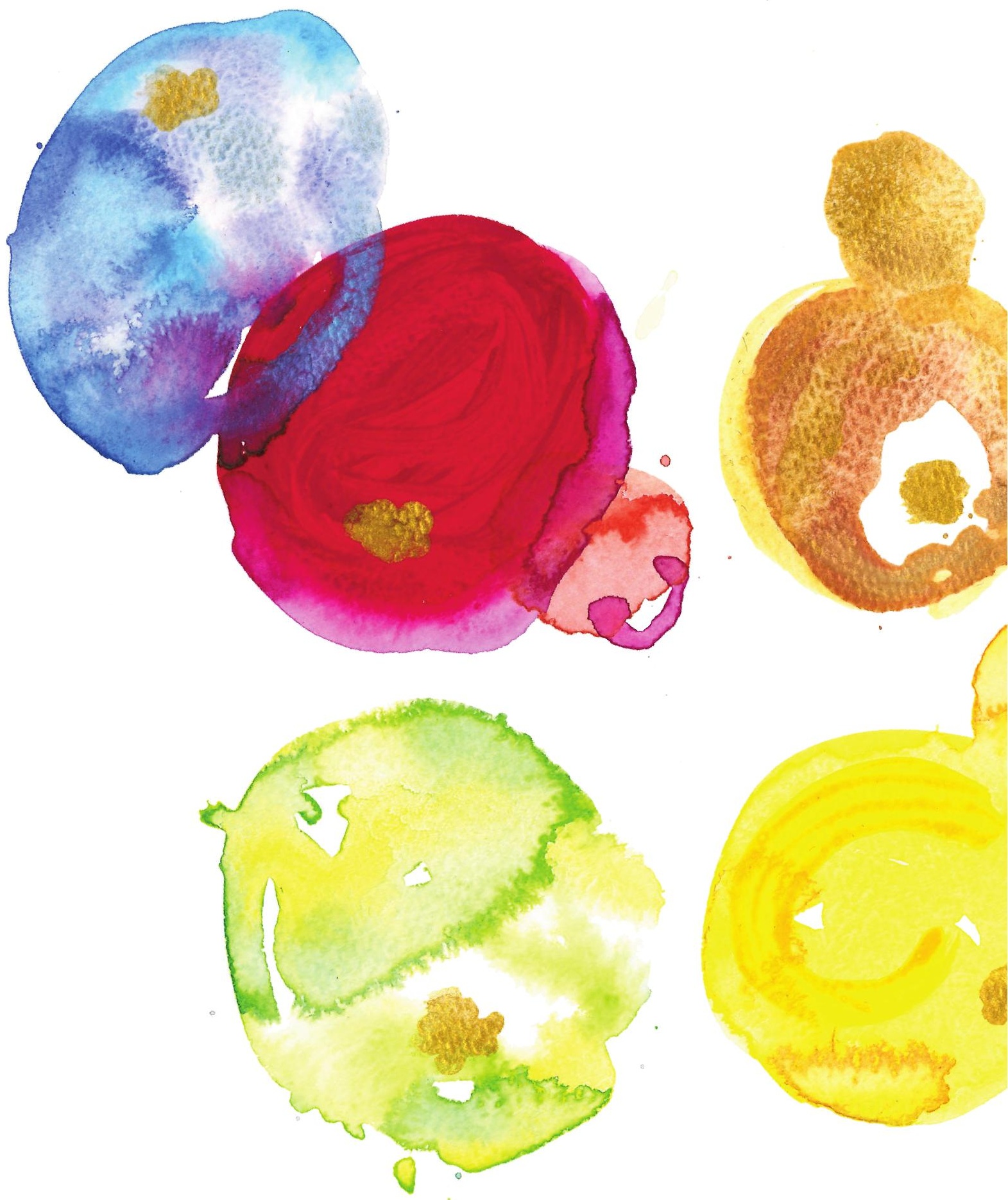


\title{
Chapter 5
}

\section{Genome-wide off-rates reveal how DNA binding dynamics shape transcription factor function}

\author{
Wim J. de Jonge, Mariël Brok, Philip Lijnzaad, Patrick Kemmeren and \\ Frank C.P. Holstege
}

Princess Máxima Center for Pediatric Oncology, Heidelberglaan 25, 3584 CS Utrecht, the Netherlands

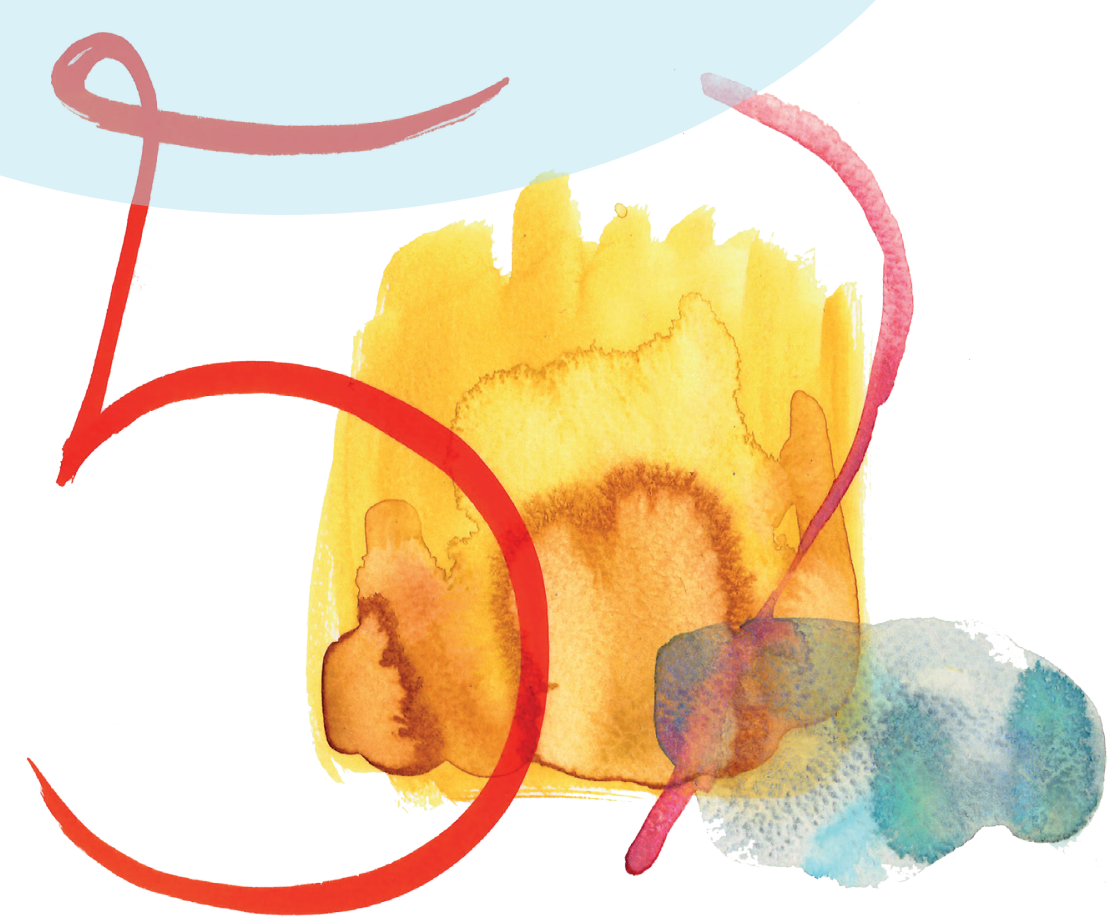

bioRxiv (2020)

doi: 10.1101/2020.05.03.074815

submitted to Molecular Systems Biology 


\section{Abstract}

Protein-DNA interactions are dynamic and these dynamics are an important aspect of chromatin-associated processes such as transcription or replication. Due to a lack of methods to study on- and off-rates across entire genomes, protein-DNA interaction dynamics have not been studied extensively. Here we determine in vivo off-rates for the Saccharomyces cerevisiae chromatin organizing factor Abf1, at 191 sites simultaneously across the yeast genome. Average Abf1 residence times span a wide-range, varying between 4.5 and 37 minutes. Sites with different off-rates are associated with different functional characteristics. This includes their transcriptional dependency on Abf1, nucleosome positioning and the size of the nucleosome-free region, as well as the ability to roadblock RNA polymerase II for termination. The results show how off-rates contribute to transcription factor function and that DIVORSEQ (Determining In Vivo Off-Rates by SEQuencing) is a meaningful way of investigating protein-DNA binding dynamics genome-wide. 


\section{Introduction}

Processes that act on chromatin, such as transcription or replication, are controlled by molecular interactions. This includes proteins interacting with DNA. ProteinDNA interactions are dynamic and these dynamics are likely important to achieve appropriate regulation of DNA-dependent processes. During transcription for example, different types of transcription factors (TFs) are continuously interacting with chromatin in a variety of ways. Each brings different functions into play: opening or closing chromatin, creating loops, modifying or evicting nucleosomes, recruiting cofactors and, in the case of activation, ultimately causing formation of a pre-initiation complex that includes RNA polymerase (Hahn \& Young, 2011; de Laat \& Dekker, 2012; Spitz \& Furlong, 2012; Struhl \& Segal, 2013; Friedman \& Rando, 2015; Kubik et al, 2017; Lai \& Pugh, 2017; Woo et al, 2017; Cramer, 2019; Brahma \& Henikoff, 2020). TFs are therefore constantly moving off and onto different loci, probing for appropriate interactions, also under conditions of steady-state transcriptional output (Hammar et al, 2014). The rates with which proteins interact with DNA, their on- and off-rates, dictate the outcome of all kinds of regulatory programs. Understanding how DNA-dependent processes work at the molecular level therefore requires methods to measure the dynamics of protein-DNA binding interactions in a systematic manner.

Different methods have been applied to investigate protein-DNA interaction dynamics. Initial in vitro measurements showed very stable TF-DNA binding that could last for more than an hour (Perlmann et al, 1990; Hoopes et al, 1992). This view was challenged by in vivo measurements showing much more dynamic interactions (Hager et al, 2009; Larson, 2011; Mueller et al, 2013; Voss \& Hager, 2014; Coleman et al, 2015; Brignall et al, 2019; Elf \& Barkefors, 2019), likely in part due to the presence of nucleosomes (Luo et al, 2014; Donovan et al, 2019a; Mivelaz et al, 2020). Direct visualization of protein-DNA interaction dynamics by fluorescence microscopy has been pivotal in forming the current view that binding of many proteins is indeed highly dynamic (McNally et al, 2000; Elbi et al, 2004; Karpova et al, 2004; Stavreva et al, 2004; Bosisio et al, 2006; Yao et al, 2006; Karpova et al, 2008; Kloster-Landsberg et al, 2012). These studies have also been crucial for showing the importance of dynamics and how this can be regulated through distinct mechanisms. A drawback of microscopy is scope however. Information is provided for only part of the nucleus collectively, or only for a single locus. It would be very useful to determine interaction dynamics at many different binding sites individually, preferably across an entire genome.

Genome-wide protein-DNA binding can be measured by chromatin immunoprecipitation (ChIP) in vivo (Gilmour \& Lis, 1984; Kuo \& Allis, 1999; Park, 2009; Collas, 2010; Furey, 2012). On its own, ChIP only provides a static indication of the degree of binding during the time-window of protein-DNA cross-linking. ChIP cannot measure protein-DNA binding dynamics directly. Competition ChIP is a ChIP variant that uses inducible switching between two differentially tagged isoforms of the same protein and has been applied to measure turnover of nucleosomes 
and TFs (Dion et al, 2007; Rufiange et al, 2007; van Werven et al, 2009; Lickwar et al, 2012; Hasegawa \& Struhl, 2019). Although limited by the induction kinetics of the competing isoform, competition ChIP has nevertheless highlighted the advantage of determining dynamics at different loci in a genome-wide manner. It has revealed differences in dynamics between promoter classes, differences in nucleosome turnover between promoters and gene bodies and showed that differential TF turnover at different loci is an important basis of transcription regulation.

Binding dynamics are determined by TF concentrations, and by on- and off-rates. Onand off-rates are two distinct facets of dynamics. Both would be useful to measure separately since they are likely influenced and regulated by different molecular mechanisms. Having both would also enable the estimation of dissociation constants and the binding free energy. A second adaptation of ChIP has indeed focused on determining on-rates by measuring the kinetics of binding during a formaldehyde cross-linking time-course (Poorey et al, 2013). As a method, this is still under development (Zaidi et al, 2017) and has only been applied to a few binding sites and not genome-wide as yet. Here, we devised a method that directly determines off-rates and does so for all binding sites across a genome. This was achieved by applying anchor-away to rapidly deplete unbound proteins from the nucleus (Haruki et al, 2008; Grimaldi et al, 2014), thereby removing the on-rate contribution to binding levels. Monitoring the time-dependent decay of protein-DNA binding across all genomic locations results in determination of off-rates, also in the form of locus-specific residence times. The method DIVORSEQ (Determining In Vivo OffRates by SEQuencing), is applied here to Abf1, a Saccharomyces cerevisiae general regulatory factor akin to chromatin pioneering TFs in mammals (Zaret \& Carroll, 2011; Kubik et al, 2017). Alongside roles in shaping chromatin architecture (Venditti et al, 1994; Lascaris et al, 2000; Yarragudi et al, 2004; Hartley \& Madhani, 2009), several different functions have been attributed to Abf1, including involvement in transcription regulation (Gailus-Durner et al, 1996), telomere binding (Enomoto et al, 1994; Pryde \& Louis, 1999), DNA replication (Marahrens \& Stillman, 1992), DNA repair (Reed et al, 1999) and RNA polymerase II roadblock termination (Roy et al, 2016; Candelli et al, 2018). Applying DIVORSEQ to Abf1 results in determination of off-rates for 191 different binding sites, with estimated residence times ranging from 4.5 to 37 minutes. Sites with different off-rates are associated with different functional characteristics that include their transcriptional dependency on Abf1, nucleosome positioning and the ability to roadblock RNA polymerase II thereby aiding transcription termination. The results emphasize that off-rate is an important characteristic of TF function and indicate that DIVORSEQ is a useful method for investigating protein-DNA binding dynamics genome-wide.

\section{Results}

\section{Nuclear depletion of Abf1}

To inducibly remove unbound Abf1 from the nucleus, an Abf1 anchor-away strain (Haruki et al, 2008) was created in the Saccharomyces cerevisiae BY4742 background (de 
Jonge et al, 2017). Abf1 was tagged with an FK506 binding protein-rapamycin binding (FRB) domain for nuclear depletion, green fluorescent protein (GFP) to monitor cellular localization and a V5 epitope for ChIP (Southern et al, 1991). ABF1 deletion is lethal (Halfter et al, 1989; Rhode et al, 1989). To investigate whether tagging of Abf1 interferes with its function, growth of tagged strains was compared to the untagged background. Tagging Abf1 has only a slight effect on growth (Supplemental Fig S1A), indicating that tagging does not greatly interfere with Abf1 function, as has been observed before (Kubik et al, 2015). Because of its essential nature, cells are expected to cease growth when Abf1 is depleted from the nucleus. Indeed, upon inducing nuclear depletion, cells show a clear disruption of growth, leading to complete growth cessation (Supplemental Fig S1B). Because loss of growth is a downstream effect, the rate of growth cessation does not necessarily reflect the speed of nuclear depletion (de Jonge et al, 2017). To directly visualize depletion, cellular localization of Abf1 was monitored using fluorescence microscopy. As expected, nuclear depletion of Abf1 indeed occurs much more rapidly than growth cessation (Supplemental Fig S1C and S1D).

\section{Determining in vivo off-rates by sequencing: DIVORSEQ}

Having ascertained Abf1 depletion, we next determined whether the system can be used to measure TF residence times $\left(1 / \mathrm{k}_{\text {off }}\right)$ at different sites across the genome. As published elsewhere (de Jonge et al, 2019), first the ChIP protocol was extensively optimized at almost all steps, to yield results better comparable between different time-points. Next, to determine off-rates, Abf1 was depleted from the nucleus and its binding levels were measured genome-wide using the optimized ChIP-seq protocol at 11 time-points during 90 minutes of depletion, all in biological triplicate (Fig 1A). The binding sites detected before depletion $(t=0)$ correspond well with previously published Abf1 binding sites (Kasinathan et al, 2014; Zentner et al, 2015; Rossi et al, 2018b). Over $90 \%$ of binding sites overlap with previously reported sites (Supplemental Fig S2A). As exemplified, different genomic locations show distinct rates of binding peak decay (Fig 1B), indicating different Abf1 residence times at these sites. Quantification and fitting the exponential decay model (Fig 1A) to the data (Fig 1C-1E), yields an estimated site-specific off-rate that can also be expressed as an average TF residence time for that site. The examples (Fig 1B-1E) were chosen to cover the wide range of different off-rates/residence times observed. After stringent peak filtering to obtain only reliable signals (Materials and Methods), off-rates and the corresponding residence times were obtained for 191 Abf1 binding sites by fitting exponential decay models to the ChIP-seq data of each individual binding peak. Almost all models closely match the actual binding data, with low residuals (Supplemental Fig S2B) and a median $R^{2}$ of 0.95 (Supplemental Fig S2C, lowest $R^{2}=0.63$ ). Based on these models the off-rates for Abf1 range between 0.027 and $0.23 \mathrm{~min}^{-1}$ (Fig 1F). This corresponds to a residence time of 4.5 minutes for the most dynamic Abf1 sites, the divergent promoter of $S R B 2$ and NCP1, and a residence time of 37 minutes for the promoter of OCA5 which has a very stable Abf1 binding peak (Fig 1G). This is the first indication that DIVORSEQ can measure residence times over a considerable range and that Abf1 has distinct residence times at different locations across the genome. 
A

Induction of depletion

$\downarrow$

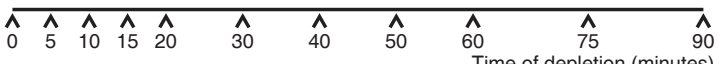

$\rightarrow \mathrm{y}(\mathrm{t})=\mathrm{y}_{\mathrm{f}}+\left(\mathrm{y}_{0}-\mathrm{y}_{\mathrm{f}}\right) \mathrm{e}^{-\mathrm{k}_{\text {off }}}$

Nuclear depletion ChIP Sequencing Binding site specific off-rates

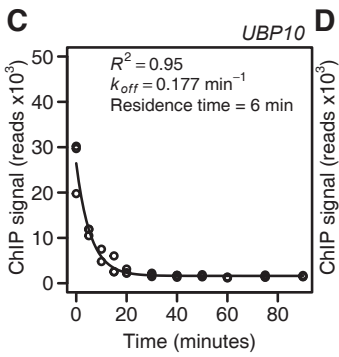

E

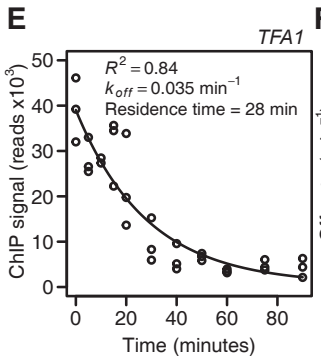

$\mathbf{F}$

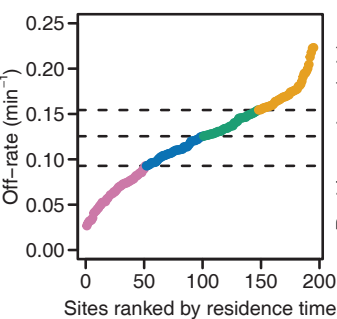

|

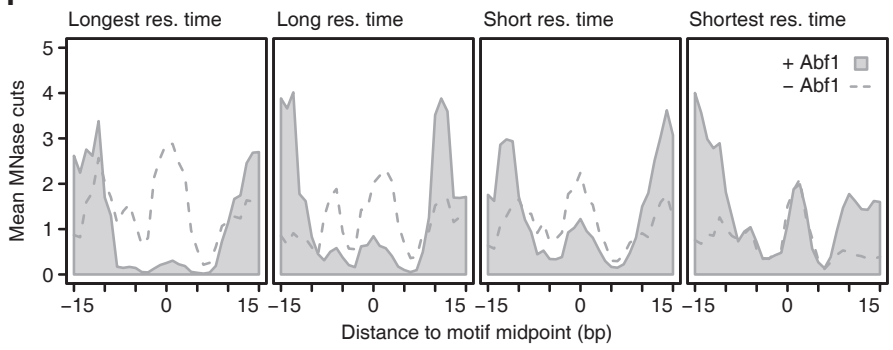

B
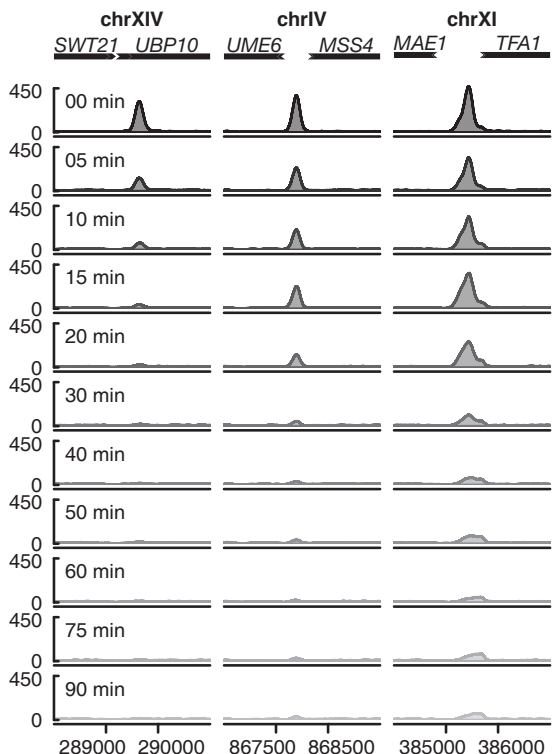

G

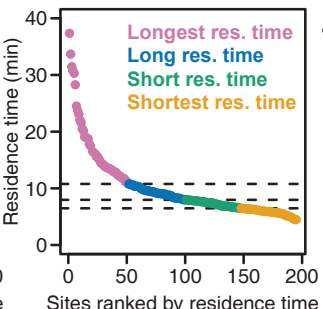

$\mathrm{H}$

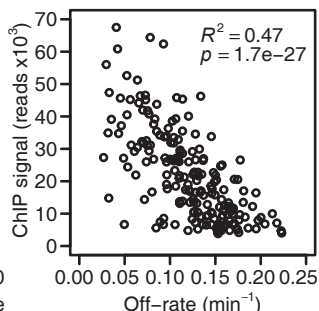

$\mathbf{J}$

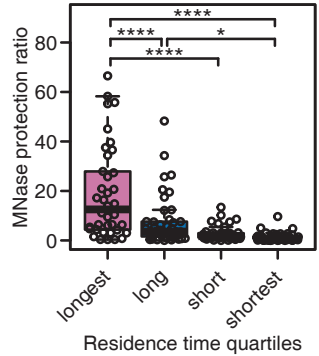

Figure 1. DIVORSEQ measures distinct residence times at different genomic sites.

(A) Schematic overview of the DIVORSEQ method. Unbound protein of interest is depleted from the nucleus and at several time points during the depletion, binding levels are measured using ChIP-seq. The decrease in binding levels is fitted using an exponential decay model and off-rates and residence times are estimated for all binding sites across the genome. (B) Abf1 binding during the depletion time course at three Abf1 binding sites with different rates of binding decrease. The signal at each time point is the average of three biological replicates, except for the 10 minutes time point, where one time point was discarded. (C-E) Fit of the exponential decay model for the examples shown in (B). The estimates for off-rates, residence time and goodness of fit are shown in the plots. (F and G) Distribution of the off-rates (F) or residence times $(\mathrm{G})$ of the 191 binding sites ranked by decreasing residence time. The four different 


\section{DIVORSEQ-derived TF residence times correspond to MNase protection rates}

To initially test whether DIVORSEQ-derived off-rates are realistic measures of Abf1 binding stability, two strategies were employed. First, residence times were compared to Abf1 binding at $t=0$. Since off-rates influence steady-state binding levels, some degree of correspondence is expected. There is indeed correlation between the DIVORSEQ-derived off-rates and binding levels (Fig $\mathbf{1 H}$ ), whereby sites with low off-rates have higher Abf1 binding levels. That the correspondence is not complete is also expected because on-rate contributes to steady-state binding levels as well. These results therefore also indicate that the relative importance of onand off-rates may differ for different genomic binding sites. A second verification of the DIVORSEQ-derived off-rates was therefore also sought. This was based on an independently generated MNase cleavage dataset, derived from a strain expressing free MNase (Kubik et al, 2018). Since it is well known that TF occupancy can result in protection against MNase cleavage, it is expected that Abf1 binding sites with the lowest off-rate should show the highest degree of MNase protection. This is indeed the case. Abf1 binding sites were divided into the four quartiles with the longest, long, short and shortest Abf1 residence times (Fig 1F and 1G). The average MNase cleavage is plotted for each quartile relative to the Abf1 binding motif (Fig 1I, grey area) and is indeed seen to increase in the four quartiles from left (longest residence times, least MNase cleavage) to right (shortest residence times, most cleavage). That protection against MNase cleavage in the quartiles with long Abf1 residence times is indeed dependent on Abf1 is demonstrated by an overall increase in MNase cleavage after prolonged Abf1 depletion (Fig 1I, dashed line). The extent of MNase protection is also shown for each individual site in each quartile (Fig 1J). DIVORSEQ-derived Abf1 off-rates correspond well to the degree of MNase protection. This indicates that the method performs as designed and provides meaningful data for a wide range of TF binding stabilities at different locations across the genome.

\section{Increased Abf1 binding stability is associated with larger nucleosome free regions}

Having established that the DIVORSEQ-derived off-rates are meaningful reflections of binding stability, we next asked whether there are mechanistic relationships between stability as measured in this manner and the roles of Abf1. Abf1 is important for shaping local chromatin architecture (Venditti et al, 1994; Lascaris et al, 2000; Yarragudi et al, 2004; Hartley \& Madhani, 2009; Ganapathi et al, 2011; Krietenstein et $a l, 2016$; Kubik et al, 2018) and contributes to the creation of nucleosome free regions

residence time quartiles are highlighted with different colours. (H) Relationship between Abf1 binding levels before depletion and off-rates for the 191 Abf1 binding sites. (I) Average in vivo MNase sensitivity of the residence time quartiles, plotted as the average number of MNase cuts at each position relative to the Abf1 binding motif (MacIsaac et al, 2006) before (grey fill) or after (dashed line) nuclear Abf1 depletion. The data were smoothed using a $3 \mathrm{bp}$ window. (J) Extent of MNase protection by Abf1 for each residence time quartile. The MNase protection ratio is the mean number of cuts in the region protected by Abf1 (-8 bp until $+8 \mathrm{bp}$ ) after depletion of Abf1 (I, dashed line), divided by the mean number of cuts in the same region before depletion (I, light grey fill). Asterisks denote a significant difference between the quartiles, calculated using a one-way ANOVA followed by Tukey's honest significant difference (HSD) test ${ }^{*} p<$ 0.05 and $\left.^{* * * *} p<0.0001\right)$. 
(NFRs) by competing with nucleosomes and acting as a barrier that chromatin remodellers use to position surrounding nucleosomes. To investigate whether Abf1 binding stability is related to its role in creating NFRs, nucleosome positioning data (Kubik et al, 2015) was investigated in the context of different Abf1 residence times. Sites with more stable Abf1 binding (longer residence times, Fig 2, top) have larger NFRs (308 bp) compared to sites with shorter residence time sites (Fig 2, bottom, 272 bp). These results obviously fit well with the idea that more stably bound Abf1 can more efficiently repel nucleosomes. However, this does not rule out the converse whereby nucleosome remodeling and nucleosome competition causes increased Abf1 off-rates at those sites with reduced residence times. Most importantly for the goals of our study, alongside the MNase protection data (Fig 1I and 1J), the NFRsize associated differences shows that DIVORSEQ-derived Abf1 off-rates can be functionally meaningful in this manner too.

\section{Changes in mRNA synthesis rates match Abf1 binding dynamics}

We next investigated whether Abf1 binding dynamics play a role in the function of Abf1 as a transcriptional regulator (Buchman \& Kornberg, 1990; Gailus-Durner et al, 1996; Miyake et al, 2002, 2004; Yarragudi et al, 2007; Paul et al, 2015; Kubik et al, 2018). Previous studies have shown that not all Abf1 bound promoters show transcriptional dependency on Abf1 (Schroeder \& Weil, 1998; Yarragudi et al, 2007; Paul et al, 2015). This has been ascribed to either lower inherent propensity for nucleosome formation at some sites, binding too far away from a transcription start site, or redundancy with other TFs (Paul et al, 2015; Kubik et al, 2018). We therefore first determined which genes are dependent on Abf1 by measuring mRNA synthesis rates genome-wide using 4-thiouracil labeling of nascent transcripts (Sun et al, 2012) during a 90 minute Abf1 depletion timecourse, at the same times points that were used to determine off-rates (Fig 3A). Approximately half of the genes with Abf1 promoter binding show a decrease in mRNA synthesis rates upon Abf1 depletion, in agreement with what has

Figure 2. Nucleosomes architecture corresponds to residence times

Average nucleosome occupancy of the residence time quartiles before (light grey fill) and after (dashed line) Abf1 depletion, centred on the Abf1 binding motif. Nucleosomes of all residence time quartiles reposition upon Abf1 depletion, which indicates that Abf1 contributes to the positioning of nucleosomes for all quartiles. The average distance between the midpoints of the -1 and +1 nucleosomes before depletion of Abf1 are indicated for each quartile. Nucleosome binding data are from (Kubik et al, 2015).

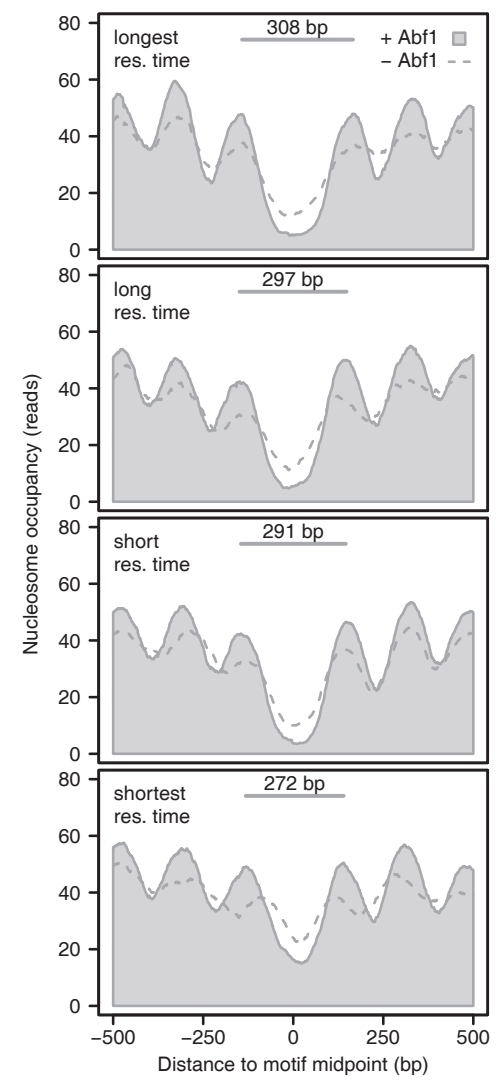


A

B

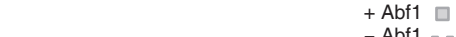

Induction of depletion - Abf1 - -
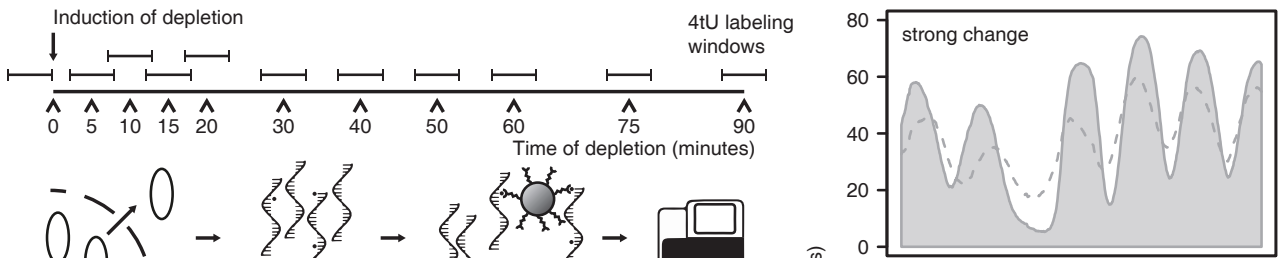

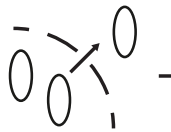

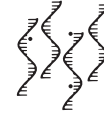

Nuclear depletion Label nascent RNA

Purify

D
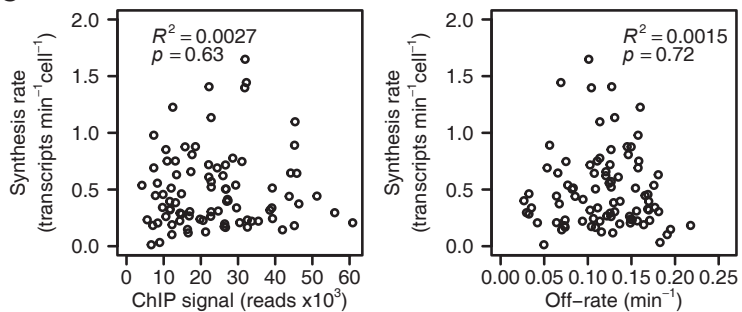

E

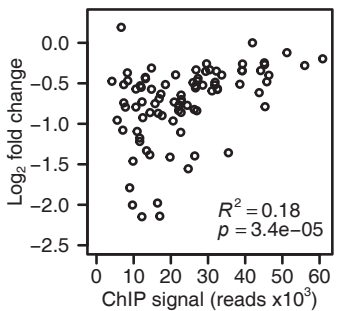

$\mathbf{F}$

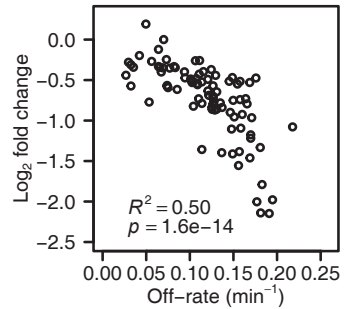

G
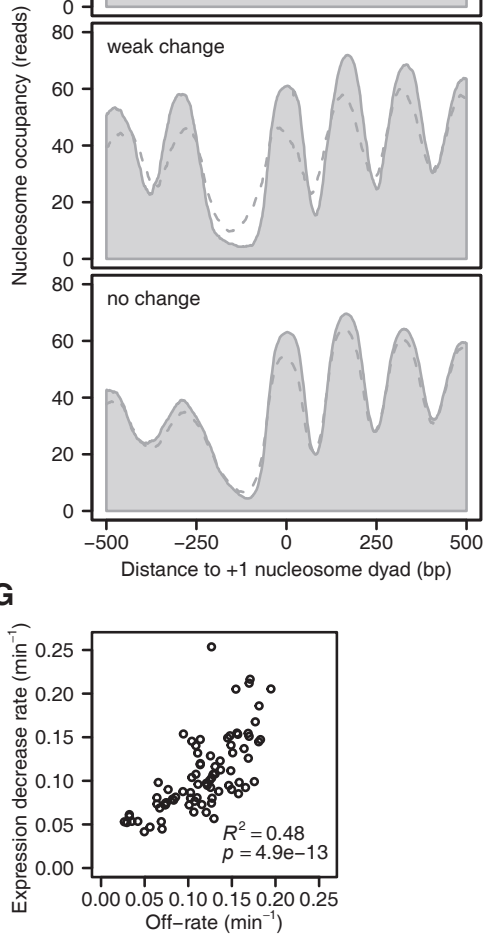

Figure 3. Dynamics of mRNA synthesis changes follow the dynamics of Abf1 dissociation.

(A) Schematic overview of the experiment set-up for measuring promoter output dynamics by labelling nascent RNA. The protein of interest is depleted from the nucleus and nascent RNA is labelled for 6 minutes using 4-thiouracil $(4 \mathrm{tU})$ at several time points during the depletion. Total RNA is extracted, nascent RNA is purified by biotinylating $4 \mathrm{tU}$ labelled RNA and the purified RNA is sequenced. Samples were taken such that the centre of the labelling period was the same as the time points that were used for DIVORSEQ. (B) Average nucleosome occupancy relative to +1 nucleosome dyad, of genes with Abf1 binding to the promoter that show strong changes (fold change $>2$ at $t=20$, top panel, $n=44$ ), weak changes ( $1.5 \leq$ fold change $\leq 2$ at $t=20$, middle panel, $n=42$ ) or no changes (fold change $<1.5$ at $t=20$, bottom panel, $n=112$ ) in mRNA synthesis upon Abf1 depletion. Nucleosome occupancy is shown before (light grey fill) and after (dashed line) Abf1 depletion. Nucleosome binding data and +1 nucleosome positions are from (Kubik et al, 2015). Downregulated and Abf1 bound genes without an annotated +1 nucleosome were omitted from the plots. (C and D) Relationship between steady state synthesis rates (Sun et al, 2012) and binding levels before depletion (C), or off-rates (D). Genes are shown that are Abf1 bound and downregulated (fold change $>1.5$ and $p<0.01$ at 20 and 30 minutes of depletion, yielding 88 genes) and have available synthesis rates ( $n=87$ ). (E and F) Relationship between $\log _{2}$ mRNA synthesis rate changes after 10 minutes of depletion and binding levels before depletion (E) or off-rates (F). Genes are shown that are Abf1 bound and downregulated (fold change $>1.5$ and $p<0.01$ at 20 and 30 minutes of depletion, $n=88$ ). (G) Relationship between expression decrease rates of downregulated genes and off-rates of the 
previously been described for Abf1 (Schroeder \& Weil, 1998; Yarragudi et al, 2007; Paul et al, 2015). The non-responsive genes show little, if any, concomitant change in nucleosome repositioning (Fig 3B), also in agreement with previous studies (Kubik et al, 2018). The set of genes that do show Abf1-dependency were next used to investigate the role of binding dynamics.

First, steady-state synthesis rates (transcripts per minute per cell, Sun et al, 2012) were compared to steady-state binding levels of Abf1. In contrast to what might be expected, there is virtually no relationship between the amount of Abf1 at a promoter and promoter activity at steady-state (Fig 3C). Regardless of absolute binding levels, promoters with more stably bound Abf1 also do not show higher synthesis rates (Fig 3D). There is however some association between the steady-state amount of bound Abf1 and the early changes in synthesis rates observed upon Abf1 depletion (Fig 3E and supplemental Fig S3A). The relationship between Abf1 presence and transcriptional dependency is markedly stronger when taking into account the DIVORSEQ-derived off-rates (Fig 3F and supplemental Fig S3B). Genes showing the largest change in promoter output are those with the highest off-rates. This holds both for the 10 minute time-point analyzed in Fig $\mathbf{3 F}$, as well as when fitting an exponential decay model to the entire mRNA synthesis rate time-course (Fig 3G). For those genes that are dependent on Abf1 for transcriptional activity, there is a strong correspondence between the loss of Abf1 and the reduction in synthesis rate. The relationship between Abf1 and transcriptional output only becomes clear when plotting off-rates (Fig 3F and 3G). This emphasizes the importance of methods to investigate interaction dynamics genome-wide and the utility of DIVORSEQ for this purpose. As discussed later, our analyses agree with the idea that an Abf1dependent NFR is the most important determinant for setting up transcription, resulting in associated dependencies on Abf1 (Paul et al, 2015; Kubik et al, 2018; Fig 3B), but that fine-tuning the absolute levels of steady-state transcriptional output is further dependent on other contributing transcription (co-)factors.

\section{Stably bound sites are more efficient roadblocks for pervasive Pol II transcription}

In addition to being a chromatin organizer, Abf1 has been shown to function as roadblock for pervasive transcription (Roy et al, 2016; Candelli et al, 2018). In this role, Abf1, like the other general regulatory factors Reb1 and Rap1, can block transcribing RNA polymerase II (Pol II). This collision causes Pol II to stall, to become ubiquitinylated and likely degraded (Colin et al, 2014; Candelli et al, 2018). An obvious hypothesis, as has indeed been suggested (Roy \& Chanfreau, 2018), is that TF binding stability may contribute to roadblock function. To test this idea, data of actively transcribing Pol II (Schaughency et al, 2014) was analyzed. The average Pol II presence relative to the Abf1 binding motif was plotted for each of the residence time quartiles (Fig $4 \mathbf{A}$ ). A roadblock peak (Fig $\mathbf{4 A}$, arrow) can be

corresponding Abf1 binding site. The expression decrease rates were calculated by fitting the $4 \mathrm{tU}$-seq time data course using the same exponential decay model that was used for the off-rates. The genes from (E and F) are shown, except for the ones where the $4 \mathrm{tU}$-seq data could not be fitted with an exponential decay model $(n=82)$. 
A

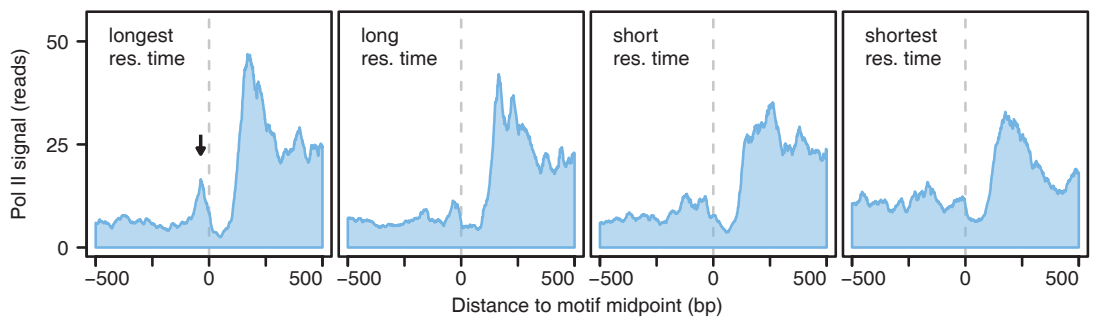

B

C
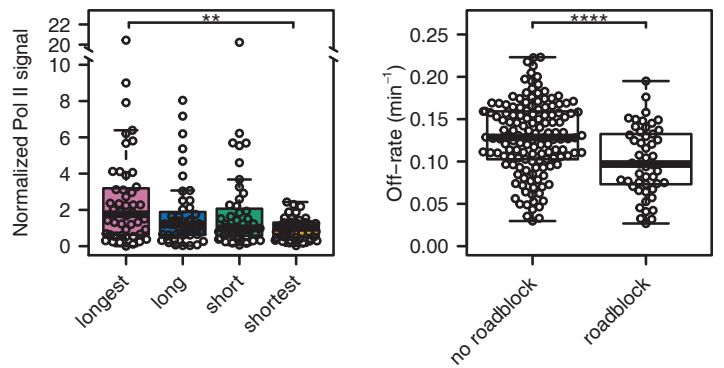

Figure 4. Sites with long residence times function as a roadblock for pervasive transcription

(A) Average RNA polymerase II binding for the different residence time quartiles. Binding profiles are centred on the Abf1 motif. The dotted line marks the midpoint of the Abf1 motif. All RNA polymerase II binding data were reoriented such that each motif is oriented in the same direction. RNA polymerase II binding is PAR-CLIP (photoactivatable ribonucleoside-enhanced cross-linking and immunoprecipitation) data from (Schaughency et al, 2014). (B) Quantification, by residence time quartile, of normalized Pol II binding levels at the roadblock peak. These levels are defined as the amount of roadblocked Pol II (located at $-37 \pm 5 \mathrm{bp}$ ) divided by the amount of upstream Pol II (from -300 to $-100 \mathrm{bp}$ ). Asterisks denote a significant difference between the quartiles, calculated using a one-way ANOVA followed by Tukey's HSD test (** $p<0.01)$. (C) Difference in off-rates between sites that are a roadblock (normalized Pol II signal $>2)$ and those that are not. The $p$-value was calculated using a two-tailed t-test $\left.{ }^{* * * *} p<0.0001\right)$.

observed immediately upstream of the Abf1 motif for the quartile with the longest residence times, and this becomes less pronounced with shorter residence times. Quantification of roadblock efficiency at each individual site confirms that stronger roadblocks are observed in the quartile with the longest residence times (Fig 4B). In agreement with this, sites with stalled Pol II have significantly lower off-rates compared to sites that do not (Fig 4C). More stably bound Abf1 is a more efficient roadblock for transcribing Pol II, further demonstrating the utility of genome-wide off-rate measurements for molecular mechanistical understanding.

\section{Factors contributing to Abf1 binding stability}

When applied to Abf1, DIVORSEQ indicates that there is a considerable range of off-rates and that this contributes to different aspects of Abf1 function. The ability to determine residence times also allows for investigation into the factors that determine different off-rates. The DNA binding motif is obviously an important factor for determining Abf1 binding stability. To evaluate the contribution of motif frequency, the number of Abf1 motifs in the vicinity of each Abf1 peak was counted. Although there are only a few peaks with multiple motifs, it is clear that most sites with more than one Abf1 binding motif have significantly longer residence times 
compared to sites with only a single binding motif (Fig 5A). Such increases in stability are likely caused by different types of cooperative effects associated with the presence of multiple motifs (Adams \& Workman, 1995; Polach \& Widom, 1996; Miller \& Widom, 2003; Hager et al, 2009; Mirny, 2010).

Beside the number of motifs, the sequence composition of the binding motif is also likely to contribute to binding stability. To investigate how motif composition affects Abf1 binding stability, the motif score of each of the binding motifs was compared between the residence time groups. Binding sites with the longest residence time have a motif that is closer to the consensus compared to the other sites (Fig $\mathbf{5 B}$ ), which indicates that having a stronger binding motif leads to more stable binding. Indeed, mutating the binding site of another well-studied TF, Gal4, results in a shorter residence time (Donovan et al, 2019b). To investigate the contribution of motif sequence to Abf1 binding stability in more detail, the consensus motifs of the different residence time groups were compared to each other (Fig 5C). Although the consensus motifs of all four groups are similar, showing good correspondence to published motifs (MacIsaac et al, 2006; Kasinathan et al, 2014; Zentner et al, 2015; Rossi et al, 2018a), the longest residence time group has a significant enrichment $(p=0.0046)$ for a thymine in the variable part of the motif, at position $-1 \mathrm{bp}$ (Fig 5C, arrow). This suggests that having a thymine at this position is not needed for binding per se, but that it contributes to the binding stability of Abf1. In agreement with this, mutation of a thymine residue at this position reduces the binding levels of Abf1 in vitro (Gailus-Durner et al, 1996).

DNA shape can also influence Abf1 binding levels (Zentner et al, 2015; Rossi et al, 2018a). Strongly and weakly bound Abf1 sites differ in their predicted minor groove width as estimated across naked DNA motifs (Rossi et al, 2018a). The Abf1 sites found here closely resemble the strongly bound sites in their minor groove width pattern (Fig 5D). Strikingly, the same analysis performed across the four groups of sites with different residence times reveals that in terms of minor groove width at position $+3 \mathrm{bp}$ (Fig 5D, arrow), the group with shortest Abf1 residence times most closely resembles the sites with low Abf1 binding levels (Rossi et al, 2018a), in having a smaller minor groove (Fig 5E). This suggests that the lower binding levels observed on sites with a reduced minor groove width at the +3 position is caused by a higher off-rate. The extended genome-wide survey of Abf1 binding stabilities demonstrates that factors influencing TF binding stability in vivo can also be advantageously studied by DIVORSEQ.

\section{Discussion}

The dynamics of proteins interacting with DNA are thought to play an important role in the regulation of chromatin-associated processes such as transcription (Hager et al, 2009). To fully understand these processes at a molecular level requires an understanding of the underlying binding dynamics. DIVORSEQ quantifies proteinDNA binding dynamics in vivo by directly measuring off-rates and residence times 
A

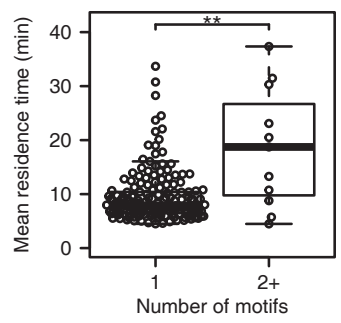

D
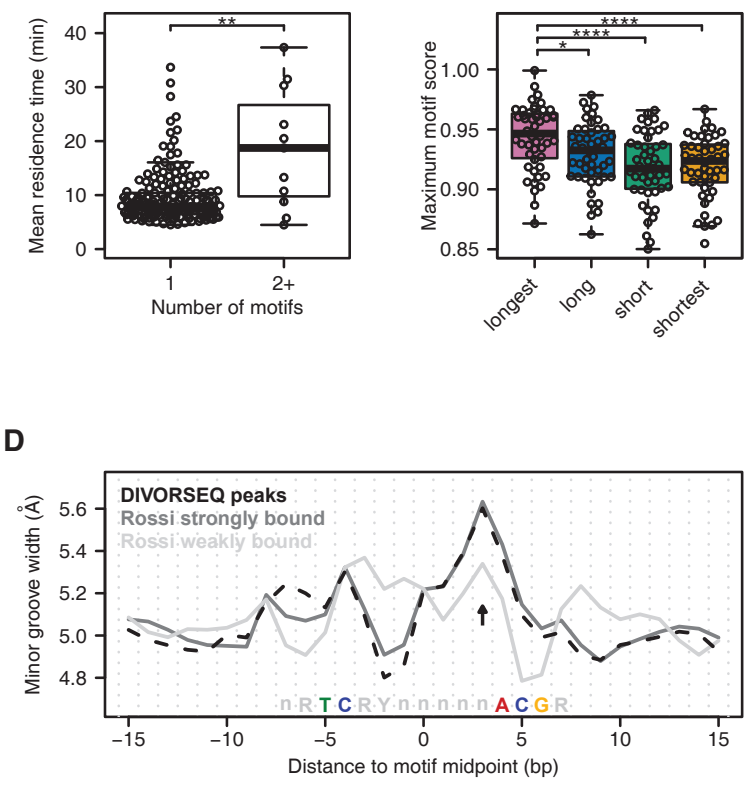

C

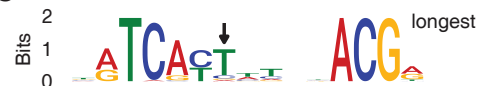

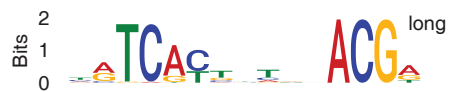

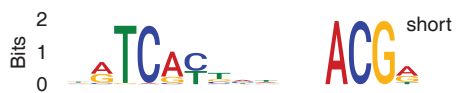

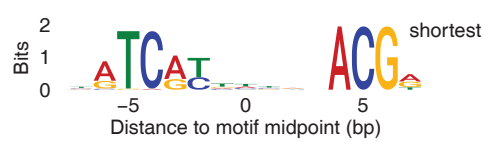

E

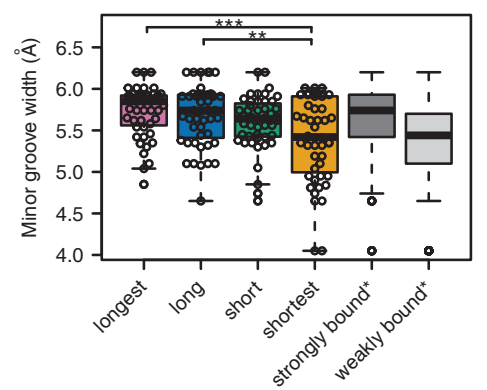

Figure 5. Factors that contribute to Abf1 binding stability.

(A) Difference in residence times between sites with one motif and sites with two or more motifs. The p-value was calculated using a Wilcoxon rank-sum test $\left({ }^{* *} p=0.0011\right)$, rather than a t-test $(p=0.0081)$ as used in Fig 4C, since the group with 1 binding motif is not normally distributed. (B) Difference in maximum motif score between the different residence time quartiles. When a site has more than one motif, the highest score was used. (C) Sequence logos showing the representative binding motif of each residence time quartile. Motifs from the longest residence time quartile are enriched $(p=0.0046)$ for having a thymine at position -1 bp (arrow). (D) Predicted minor groove width centred at the Abf1 binding motif of all 191 Abf1 sites found here (black, dashed line) and Abf1 binding motifs defined as strongly bound (dark grey line) and weakly bound (light grey line) by (Rossi et al, 2018a).(E) Difference in predicted minor groove width between the residence time quartiles at position $+3 \mathrm{bp}$ from the motif midpoint $(\mathrm{D}$, arrow). In addition, the minor groove width at this position of the Abf1 motifs defined as strongly and weakly bound by (Rossi et al, 2018a) are shown ( $n=400$ for each group). Asterisks in (B) and (E) denote adjusted $p$-values calculated using a one-way ANOVA followed by Tukey's HSD test between the residence time quartiles $\left({ }^{*} p<0.05,{ }^{* *} p<0.01,{ }^{* * *} p<0.001\right.$, $\left.{ }^{* * * *} p<0.0001\right)$.

at multiple binding sites across an entire genome. Applying DIVORSEQ to the TF Abf1 shows that the method can measure meaningful differences in off-rates spanning a wide range of values. Our results show how motif number, sequence and structure of the binding motif contribute to off-rates and how this aspect of binding dynamics influences the roles of Abf1 as a chromatin organizing factor, a transcriptional regulator and a termination roadblock for RNA polymerase II at different sites across the genome.

Abf1 is a general regulatory factor, known to organize chromatin (Venditti et al, 1994; Lascaris et al, 2000; Yarragudi et al, 2004; Hartley \& Madhani, 2009; Ganapathi et al, 2011; Krietenstein et al, 2016; Kubik et al, 2018). Our results show good 
correspondence between NFR size and binding stability (Fig 2). Sites with shorter Abf1 residence times have smaller NFRs. This could either be explained by stronger nucleosome exclusion through more stably bound Abf1, or conversely, by more stable binding of Abf1 in larger NFRs. Abf1 shapes the local chromatin architecture by competing with nucleosomes (Venditti et al, 1994; Yarragudi et al, 2004) and by acting as a barrier that chromatin remodellers use to position flanking nucleosomes (Krietenstein et al, 2016). It seems reasonable that Abf1 forms a more efficient barrier when it is more stably bound, thus repelling nucleosomes more efficiently, which would support the hypothesis that stable binding creates bigger NFRs. On the other hand, being a barrier means that chromatin remodelers actively position nucleosomes towards Abf1. Therefore, nucleosomes that are being positioned by remodelers may exert a force on Abf1 and destabilize its binding. In this hypothesis, competition with nucleosomes could reduce the residence time of Abf1, as has been shown for Rap1 (Lickwar et al, 2012; Mivelaz et al, 2020). Upon depletion of Abf1, nucleosomes become repositioned in all Abf1 residence time quartiles, but sites with the shortest residence time show the biggest reduction in NFR size (Fig 2). This fits with nucleosome positioning leading to shorter Abf1 residence times at these sites. Neither hypothesis can, nor need be excluded as yet. The observed correspondence between NFR size and Abf1 off-rates at different sites highlights the advantage of such measurements as a starting point for detailed characterization of molecular mechanisms.

Besides organizing chromatin, Abf1 also functions as a transcriptional regulator (Buchman \& Kornberg, 1990; Gailus-Durner et al, 1996; Miyake et al, 2002, 2004; Yarragudi et al, 2007). Abf1 is known to be only a weak activator of transcription (Buchman \& Kornberg, 1990; Levo et al, 2017) and the results presented here indicate that it mainly regulates transcription through repositioning nucleosomes, which is consistent with previous reports (Paul et al, 2015; Kubik et al, 2018). Our results fit with Abf1 stimulating transcription by creating an NFR that allows other regulatory factors to bind and whose activities may more directly dictate steady-state expression levels. This offers an explanation for why there is little correlation between steadystate mRNA synthesis rates and Abf1 binding levels or off-rates (Fig 3C and 3D), but nevertheless good correlation between off-rates and changes in mRNA synthesis rates upon depletion (Fig 3F). Removal of Abf1 causes NFR collapse for those promoters that have no redundant mechanisms of NFR upkeep (Fig 3B), resulting in cessation of promoter activity. This is in contrast to Rap1, which is known to directly contact TFIID (Garbett et al, 2007) and may directly recruit the transcription pre-initiation complex itself. Such direct recruitment suggests that Rap1 is the main regulator of transcription of its targets, explaining correlation between Rap1 binding dynamics and steady-state mRNA synthesis levels (Lickwar et al, 2012). As with the analysis of roadblock function for Abf1, different modes of regulator activity or function may therefore be revealed by detailed analyses of binding dynamics.

A limitation of DIVORSEQ is that analysis is in bulk, rather than at the single cell resolution available through microscopy (Hager et al, 2009; Larson, 2011; Mueller et al, 2013; Voss \& Hager, 2014; Coleman et al, 2015; Brignall et al, 2019; 
Elf \& Barkefors, 2019). This is offset by the advantage of determining off-rates for many loci across the genome in parallel. Other methods that measure site-specific in-vivo binding dynamics include competition ChIP, which determines turnover and is limited by a slow induction of the competitor protein, as well as by the substantial carbon source perturbation required for induction (Schermer et al, 2005). DIVORSEQ directly measures off-rates and has been designed for application alongside on-rate measurement by cross-linking kinetic analyses (Poorey et al, 2013), that has yet to be applied at the genomic scale (Zaidi et al, 2017). Considerations that need to be made when applying DIVORSEQ include having sufficiently rapid removal of unbound proteins from the nucleus. Using anchor-away (Haruki et al, 2008), an estimated 2,000 molecules can be depleted from the nucleus per minute (Warner, 1999). This implies that for highly abundant proteins such as Abf1, with an estimated 6,000 molecules (Ho et al, 2018), residence times will be determined at minute-scale resolution. That anchor-away is sufficiently rapid for Abf1, is indicated by the excellent fit to first-order kinetics observed and the wide range of different off-rates obtained. Besides anchor-away, other techniques that facilitate nuclear depletion could also be used (Klemm et al, 1997; Bayle et al, 2006; Busch et al, 2009), contingent on rapidity. A second consideration is that the ChIP or genomic location protocol requires results that are comparable between time points. Here we first extensively optimized almost all ChIP protocol steps to achieve this (de Jonge et al, 2019). Some limitations still remain. Abf1 can only be cross-linked to sites with a guanine or cytosine at $-8 \mathrm{bp}$ from the binding motif center (Rossi et al, 2018a). Sites without a guanidine or cytosine at this position were therefore excluded here although some nevertheless yielded low signals, likely caused by small amounts of cytosolic Abf1 rebinding during the ChIP procedure. Combined with peak filtering for robust binding, this reduced the number of Abf1 sites for which off-rate could be determined. Improvements to DIVORSEQ could therefore be aimed at preventing rebinding and/or applying assays that do not depend on cross-linking (Zentner et al, 2015; Skene \& Henikoff, 2017). These considerations aside, that the determined offrates are accurate is corroborated by the MNase protection levels at Abf1 sites with different off-rates, as well as by the diverse aspects of previously established Abf1 function presented here for the first time in the context of a large number of genomic binding sites and their binding dynamics.

\section{Materials and Methods}

\section{Strains}

The strains used in this study are Saccharomyces cerevisiae anchor-away strains (Haruki et al, 2008) that were recreated in the BY4742 background (de Jonge et al, 2017). Besides the FRB domain from mammalian target of rapamycin (mTOR), the anchor-away tag consists of yeast enhanced green fluorescent protein (yEFGP) and a triple V5 tag. The parental BY4742 anchor-away strain, which has an FPR1 deletion and a tor 1-1 mutation to desensitize the strain to rapamycin, was used as a wildtype control. 


\section{Growth conditions}

Strains were streaked from $-80^{\circ} \mathrm{C}$ stocks on appropriate selection plates (for the parental anchor-away strain: YPD + Nourseothricin and for the Abf1-aa strains: YPD + Nourseothricin + Hygromycin), and incubated at $30^{\circ} \mathrm{C}$ for 3 days. In the morning, liquid pre-cultures were inoculated in $1.5 \mathrm{ml}$ of synthetic complete (SC) medium: $2 \mathrm{~g} / \mathrm{l}$ dropout mix complete and $6.71 \mathrm{~g} / \mathrm{l}$ yeast nitrogen base without amino acids, carbohydrate \& w/AS (YNB) from US Biologicals (Swampscott, USA) with $2 \%$ D-glucose. In the afternoon, several pre-cultures were combined, diluted to final volume of $20 \mathrm{ml}$ and grown overnight. The growth conditions were identical for all experiments and pre-cultures: in SC medium at $30^{\circ} \mathrm{C}$, with shaking (230 rpm).

\section{Anchor-away depletion}

At $t=0$, Abf1 was depleted from the nucleus by addition of rapamycin (LC Laboratories \#R-5000; dissolved to $2 \mathrm{mM}$ in DMSO), to a final concentration of $7.5 \mu \mathrm{M}$. For the $t=0$ time point, the same volume of DMSO instead of rapamycin was added and incubated for 90 minutes.

\section{Chromatin immunoprecipitation}

ChIP was performed as described in detail in (de Jonge et al, 2020) using biological triplicates. To summarize: cells were diluted in the morning to an optical density (OD) of 0.11-0.15 (WPA Biowave CO8000 Cell Density Meter) in $100 \mathrm{ml}$ of SC medium, and grown for at least 2 doublings to an $\mathrm{OD}_{600}=0.8$, which corresponds to about $2 \times 10^{7}$ cells per ml. Additions of rapamycin and DMSO were staggered such that all time points were ready at the same OD (0.8). When this OD was reached, the cells were cross-linked for 5 minutes by addition of $37 \%$ formaldehyde (SigmaAldrich \#252549) to a final concentration of $2 \%$. The formaldehyde was quenched using a final concentration of $1.5 \mathrm{M}$ of Tris (tris(hydroxymethyl)aminomethane) for 1 minute. Subsequently, the cells were pelleted by centrifugation at $3220 \mathrm{~g}$ at $4^{\circ} \mathrm{C}$ for 3 minutes. The pellet was washed in $10 \mathrm{ml}$ TBS $(150 \mathrm{mM} \mathrm{NaCl}, 10 \mathrm{mM}$ Tris $\mathrm{pH}$ 7.5) and pelleted again at $3220 \mathrm{~g}$ for 3 minutes at $4^{\circ} \mathrm{C}$. After resuspension in $1 \mathrm{ml} \mathrm{MQ}$, cells were centrifuged at $3381 \mathrm{~g}$ for 20 seconds at room temperature and the pellet was snap-frozen in liquid nitrogen and stored at $-80^{\circ} \mathrm{C}$.

To lyse cells, the cells were resuspended in FA lysis buffer (50 mM HEPES-KOH pH 7.5, $150 \mathrm{mM} \mathrm{NaCl}, 1 \mathrm{mM}$ EDTA pH 8.0, $1 \%$ Triton X-100, 0.1\% Na-deoxycholate, $0.1 \%$ SDS) containing the protease inhibitors aprotinin, pepstatin A, leupeptin and PMSF to a final volume of $2 \mathrm{ml}$, transferred to 2 - $\mathrm{ml} \mathrm{screw-cap} \mathrm{tubes} \mathrm{and} \mathrm{disrupted} \mathrm{using}$ zirconium/silica beads $0.5 \mathrm{~mm}$ (BioSpec Products, \#11079105z) by bead beating 7 times 3 minutes in a Genie Disruptor (Scientific Industries). The lysate was recovered and centrifuged at $1503 \mathrm{~g}$ for 2 minutes at $4^{\circ} \mathrm{C}$ to remove cell debris. The supernatant was subsequently fragmented by sonicating the samples for 10 cycles of 15 seconds on, 30 seconds off using a Bioruptor Pico sonicator (Diagenode \#B01060010).

For the immunoprecipitation, $450 \mu \mathrm{l}$ of the fragmented chromatin was incubated with $1 \mu \mathrm{l}$ of anti-V5 antibody (Life Technologies \#R96025) for 2 hours at $4^{\circ} \mathrm{C}$. A $20 \mu \mathrm{l}$ aliquot was kept separate as an input control. The chromatin + antibody were subsequently 
bound for 20 minutes at room temperature to magnetic beads (Dynabeads protein G, Life Technologies \#10004D) that were pre-incubated with BSA. The beads were washed twice with PBS and twice using PBS-T. During the last wash, the beads were transferred to fresh LoBind tubes (Eppendorf \#0030108051). Cross-links were reversed by incubating in TE/1\% SDS (10 mM Tris pH 8.0, 1 mM EDTA pH 8.0, 1\% SDS (w/v)) overnight at $65^{\circ} \mathrm{C}$. The next morning, RNA was degraded by addition of RNAse A/T1 (Thermo Scientific \#EN0551) at $37^{\circ} \mathrm{C}$, and subsequently proteins were digested by addition of proteinase $\mathrm{K}$ (Roche $\# 03115852001$ ) at $37^{\circ} \mathrm{C}$. After protein digestion, DNA was recovered using a Qiagen PCR purification cleanup kit (Qiagen \#28106) by eluting in $30 \mu \mathrm{l}$ buffer EB.

\section{RNA labeling and extraction}

For the 4tU-seq time course, $20 \mathrm{ml}$ cultures were used, with biological triplicates for each time point. Rapamycin and DMSO additions were staggered such that all cultures were ready at the same OD (0.8). WT samples incubated with rapamycin or DMSO for 90 minutes were taken along as a controls. Three minutes before the cultures were ready, 4-thiouracil (4tU; Sigma-Aldrich \#440736) was added to the cell cultures to a final concentration of $5 \mathrm{mM}$. Cells were incubated with $4 \mathrm{tU}$ for 6 minutes in total, such that the center of the labeling period matched the time point of the ChIP time course. Subsequently, cells were harvested by centrifugation at $3220 \mathrm{~g}$ for 3 minutes, cell pellets were snap frozen immediately in liquid nitrogen and stored at $-80^{\circ} \mathrm{C}$.

To isolate total RNA, cells were resuspended in Acid Phenol Chloroform (Sigma \#P1944) and immediately mixed with the same volume of TES buffer (10 mM Tris $\mathrm{pH}$ 7.5, $10 \mathrm{mM}$ EDTA, 0.5\% SDS). The samples were vortexed hard for 20 seconds, the tubes were covered in aluminum foil to keep the samples dark, and incubated in a $65^{\circ} \mathrm{C}$ water bath for 10 minutes. Next, the samples were transferred to $1.5 \mathrm{ml}$ tubes and incubated in a thermomixer for 50 minutes at $65^{\circ} \mathrm{C}$ and $1400 \mathrm{rpm}$, while covered in aluminum foil. After incubation, samples were centrifuged at $18407 \mathrm{~g}$ for 20 minutes at $4^{\circ} \mathrm{C}$. The water phase was recovered and phenol extraction was repeated once, followed by extraction using chloroform-isoamyl-alcohol (25:1). The RNA was precipitated using sodium acetate (NaAc 3M, pH 5.2) and 100\% ethanol $\left(-20^{\circ} \mathrm{C}\right)$ by incubating at $-20^{\circ} \mathrm{C}$ for at least $30 \mathrm{~min}$. DTT was also added to a final concentration of $1 \mathrm{mM}$ to prevent oxidation of the $4 \mathrm{tU}$. The pellet was washed once with $80 \%$ ethanol and resuspended to a final concentration of $1 \mu \mathrm{g} / \mu \mathrm{l}$ in sterile MQ. To recover nascent transcripts the protocol from (Dölken et al, 2008) was used with minor adaptations. In brief, $100 \mu \mathrm{g}$ of cleaned RNA was heated to $60^{\circ} \mathrm{C}$ for 10 minutes and immediately put on ice for 2 minutes. The RNA was biotinylated by adding $200 \mu \mathrm{l}$ Biotin-HPDP (Thermo Fisher Scientific \#21341) dissolved to $1 \mathrm{mg} / \mathrm{ml}$ in 30\% DMF. Unbound biotin was removed using chloroform extraction. Biotinylated RNA was separated from total RNA using streptavidin-conjugated magnetic beads and $\mu \mathrm{MACs}$ columns (Miltenyi Biotec \#130-074-101). The beads were washed 6x using $65^{\circ} \mathrm{C}$ washing buffer (100 mM Tris pH 7.5, 10 mM EDTA, $1 \mathrm{M} \mathrm{NaCl}, 0.1 \%$ Tween-20) and bound RNA was eluted using $200 \mu \mathrm{l}$ of $100 \mathrm{mM}$ DTT. The nascent RNA was purified using an RNeasy MinElute Cleanup Kit (Qiagen \#74204). 


\section{Library preparation and sequencing}

ChIP-seq libraries were created using a combination of a NEXTflex Rapid DNASeq Kit (Bioo Scientific \#NOVA-5144) and a NEXTflex Rapid Directional qRNA-Seq Kit (Bioo Scientific \#NOVA-5130) to allow for incorporation of unique molecular identifiers (UMIs) (Kivioja et al, 2012) on both sides of each fragment. To improve speed and accuracy, a maximum of 8 libraries were prepared at the same time. To make the amount of starting material of the input samples similar to that of the IP samples, the input samples were diluted 1:300 prior to library prep. End-repair and adenylation were carried using the NEXTflex Rapid DNA-Seq Kit with half of the recommended volumes. The subsequent steps were carried out using the NEXTflex Rapid Directional qRNA-Seq Kit with a quarter of the recommended volumes for the adapter ligation and half volumes for the PCR amplification. The initial volume used for each of the bead clean-ups was adjusted to $50 \mu \mathrm{l}$ by addition of MQ and bead ratios were kept as recommended. The number of PCR cycles used was the same for all ChIP and input samples (13 cycles) except for the WT IPs where 15 PCR cycles were used. Library yields were assessed using a High Sensitivity DNA bioanalyzer chip (Agilent) and equimolar amounts of library were pooled and sequenced paired-end 2x 75bp on a NextSeq 500 system (Illumina).

4tU-seq libraries were created using the NEXTflex Rapid Directional qRNA-Seq Kit (Bioo Scientific \#NOVA-5130) with a slightly modified protocol. During step $D$ of the protocol (i.e. bead cleanup after second strand synthesis) the beads were resuspended in $10 \mu \mathrm{l}$ of resuspension buffer, and $8 \mu \mathrm{l}$ was used for the next step. From this step onwards, half of all recommended volumes were used. The initial volume used for each of the bead clean-ups was adjusted to $50 \mu \mathrm{l}$ by addition of MQ and bead ratios were kept as recommended. Since the concentrations of labelled RNA differed after the purification, a qPCR was performed to estimate the number of PCR cycles needed for each sample after adapter ligation. The number of cycles that were used varied between 8-12 cycles. Library yields were assessed using a High Sensitivity DNA bioanalyzer chip (Agilent) and equimolar amounts of library were pooled and sequenced paired-end $2 \times 75 \mathrm{bp}$ in two sequence runs on a NextSeq 500 system (Illumina).

\section{Mapping}

Reads from both the ChIP-seq and 4tU-seq experiments were aligned to the sacCer3 genome assembly (February 2011) using HISAT2 v2.0.5 (Kim et al, 2015). The settings for the ChIP-seq samples were "--add-chrname -X 1000 --scoremin L, 0,-0.2 -k 1 --no-spliced-alignment $-5 \quad 12-36^{\prime \prime}$ and for the 4tU-seq samples the settings "--add-chrname -X 1000 --score-min L, 0,$0.175 \quad-5 \quad 10-3 \quad 10$--dta -max-intronlen 1500 --rna-strandness $\mathrm{RF}^{\prime \prime}$ were used. Subsequently, the bam files were filtered to keep only transcripts with a unique combination of UMIs using the custom scripts "addumis2bam.sh" and "uniqify-umis.pl" available from https://github.com/wdejonge/DIVORSEQ.

\section{Peak calling and filtering}

The ChIP-seq data was filtered to keep only uniquely mapping reads and 
subsequently peaks were called using MACS2 v2.1.1.20160309 (Zhang et al, 2008) with the settings "-f BAMPE -g $1.25 e 7$--keep-dup all --mfold 52000 --call-summits -q 0.001 --fe-cutoff 2 " using all three replicate $t=0$ time points (no depletion) versus their corresponding inputs, yielding 948 Abf1 peaks.

To monitor the depletion, the initial binding levels need to be sufficiently strong to accurately measure a reduction in binding levels. Therefore, only binding peaks with a fold enrichment of at least 4 were considered (421 peaks). This also filters out apparent weak binding across open reading frames and tRNAs, which is a known artefact of ChIP (Park et al, 2013; Teytelman et al, 2013). In addition, it has been recently shown that Abf1 is only efficiently cross-linked to sites with either a guanine or a cytosine at $-8 \mathrm{bp}$ from the center of the Abf1 binding motif (Rossi et al, 2018a). For each binding peak, we searched for motifs ( $\pm 100 \mathrm{bp}$ from peak summit) that closely match the Abf1 consensus (at least $85 \%$ of the consensus motif score from (MacIsaac et al, 2006)) and determined whether there was a G/C or an A/T at $-8 \mathrm{bp}$ from each motif. Only peaks where all motifs found had a G/C at $-8 \mathrm{bp}$ from the motif midpoint were kept for further analysis (195 peaks). Four of these peaks were located in telomeric regions. Although the residence time estimates of these four peaks are probably accurate, we noticed that other characteristics were very distinct from other peaks (e.g. nucleosome organization, motif composition, RNA polymerase II binding). Therefore, these peaks were excluded, yielding a total of 191 Abf1-binding sites that were analyzed in greater detail.

\section{Exponential decay fitting}

To fit the exponential decay model, first the bam files were centered and smoothed with a 101bp window, as described in (de Jonge et al, 2017). Subsequently, all samples were scaled to 1 million reads using genomecov from the bedtools2 suite v2.27 (Quinlan \& Hall, 2010), which makes sure that total coverage across the genome is the same for all samples $\left(1.01 \times 10^{8}\right.$ bases $)$. For each binding site $(n=191)$ the total coverage was calculated for each sample in a window $\pm 50 \mathrm{bp}$ from the peak summit. These values were used to fit a first-order exponential decay function using the $n 1 \mathrm{~s}$ function in R:

$$
y(t)=y_{f}+\left(y_{0}-y_{f}\right) e^{-k_{o f f} t}
$$

with $y_{0}$ the binding levels a $t=0$ (i.e. before depletion), $t$ the time since depletion, $y_{f}$ the final binding level and $k_{\text {off }}$ the decay rate of the binding levels. The residence time is given by $1 / k_{\text {off }}$ and represents the average time Abf1 stays bound to a specific site. The fits were done in $\mathrm{R}$ with the nls function using the formula: "nls (ChIP SSasymp (time, yf, y0, log_koff), data = data)", yielding regressions with excellent $R^{2}$, with the lowest $\bar{R}^{2}=0.63$ and the median $R^{2}=0.95$ (Supplemental Fig S2C). One of the 10 minute depletion ChIP samples had much higher binding levels compared to the other 10 minutes samples, with a median absolute deviation more than six times as high. Upon removal of this sample all fits improved. This sample was therefore removed from all analyses. The 191 binding sites were divided into four residence time groups defined by the quartiles of their residence times (48 
or 47 sites per quartile).

\section{4tU-seq expression analysis}

The 4tU-seq reads were assigned to genomic features using featureCounts from the subread package v1.6.5 (Liao et al, 2014). As an annotation file, transcription start site annotations from (van Bakel et al, 2013) were merged with the genome annotation from the Saccharomyces genome database (SGD) (Cherry et al, 2012) containing ORFs, tRNAs, rRNAs and snRNAs. The counts from the two independent sequence runs were combined and differential expression of the genomic features (genes) was calculated using the DESeq2 package v1.10.1 (Love et al, 2014) in R. Only genes with a fold change of more than 1.5 with an adjusted $p$-value $<0.01$ after 20 minutes as well as 30 minutes of depletion were considered differentially expressed. The fold change in mRNA synthesis was calculated relative to $t=0$.

To model the decrease rate of mRNA synthesis, absolute transcript counts were used at each time point. They were normalized to the median number of transcripts of all samples after filtering out rRNAs. Only genes that were significantly downregulated with Abf1 binding to the promoter were used $(n=88)$. Binding peaks were assigned to genes when the summit of that peak was found within the promoter $(500 \mathrm{bp}$ upstream of the transcription start site). As described for the ChIP-seq data, a firstorder exponential decay function (Eq. 1) was used to model the changes in mRNA synthesis. In this case $y_{0}$ is the expression level before depletion and $k_{\text {off }}$ is the rate with which the expression decreases to the final expression level $y_{f}$. For six of the genes with robust changes in mRNA synthesis no reliable fit could be obtained and these were therefore excluded from Fig 2G $(n=82)$.

\section{External datasets}

To assess what percentage of Abf1 binding peaks overlap with previously detected Abf1 binding sites, the peaks (peak summits $\pm 50 \mathrm{bp})$ detected here $(n=948)$ were compared to published data sets. Data from three different techniques were used: ORGANIC, ChEC-seq and ChIP-exo (Kasinathan et al, 2014; Zentner et al, 2015; Rossi et al, 2018b). For the ORGANIC data the published bound Abf1 sites $(n=1068)$ from the "10' MNase $80 \mathrm{mM}^{\prime}$ " samples were used (Kasinathan et al, 2014). For the ChECseq data the Abf1 sites that were both classified as "fast" and "high scoring motif" $(n=1583)$ were taken (Zentner et al, 2015). For the ChIP-exo peaks, all sites detected using the ChIP-exo protocol v5 ( $n=3177)$ were used (Rossi et al, 2018b).

To assess in vivo protection from MNase cleavage, the bigwig files "Abf1aa_V freeMNase_ChEC" and "Abf1aa_R_freeMNase_ChEC" were downloaded from the gene expression omnibus (GEO) dataset GSE98259 (Kubik et al, 2018) and smoothed with a $3 \mathrm{bp}$ window. The data were centered on the Abf1 binding motif and the average number of cleavage sites per residence time quartile were calculated. Subsequently, the average number of cut sites was calculated in the area of the motif ( $-8 \mathrm{bp}$ to $+8 \mathrm{bp}$ from the motif midpoint) both in the presence and absence of Abf1. The cleavage ratio was calculated by taking the number of cuts in the absence of Abf1 divided by the number of cuts in the presence of Abf1. Sites without cuts in either conditions (with or without rapamycin) were excluded from the quantification (Fig 
1J).

To compare synthesis rates with off-rates, residence times and binding levels, genome-wide synthesis rates were taken from (Sun et al, 2012), by downloading them from the researchers' website: https:/www.mpibpc.mpg.de/13760807/Sc_ turnover.zip.

To visualize nucleosome positioning before and after depletion of Abf1, the bigwig files "Abf1veh15" and "Abf1rapa15" were downloaded from GEO dataset GSE73337 (Kubik et al, 2015). The data were either centered on the Abf1 binding motif (Fig 2) or aligned on the +1 nucleosome (Fig 3B), with +1 nucleosome positions taken from (Kubik et al, 2015). The average nucleosome occupancy was calculated for genes with Abf1 binding, an annotated +1 nucleosome and that were strongly downregulated (fold change $>2$ at $t=20, n=44)$, weakly downregulated $(1.5<$ fold change $<2$ at $t=20$, $n=42$ ) or did not change (fold change $<1.5$ at $t=20, n=112$ ) upon depletion of Abf1 (Fig 3B). In Fig 2 the average nucleosome occupancy was calculated per residence time quartile.

To visualize polymerase binding, the PAR-CLIP (photoactivatable ribonucleosideenhanced cross-linking and immunoprecipitation) data for both the plus and minus strand from sample "Rpb2-HTB Control With Rapamycin" were downloaded from GEO dataset GSE56435 (Schaughency et al, 2014). As the Abf1 motif is strandspecific, the data were reoriented accordingly, meaning that when the data had to be reoriented to match the orientation of the motif (as shown in Fig 5C), the plus and minus strand were also swapped. To calculate the roadblock efficiency, the average binding in the roadblocked peak, located at -37 bp \pm 5 bp from the Abf1 binding motif center, was normalized by the amount of incoming transcription (defined as the average Pol II binding in the region from -300 bp until -100 bp upstream of the Abf1 binding motif). For the quantification shown in Fig 4C, a peak was considered to be a roadblock peak when it had upstream normalized Pol II binding $>2$. With this cut-off approximately $25 \%$ of the peaks (49/191) were considered to be a roadblock for Pol II.

\section{Motif scoring and DNA shape analysis}

The position frequency matrix from (MacIsaac et al, 2006) was obtained from the YeTFaSCo database (Boer \& Hughes, 2011), multiplied by a factor 1000 and converted to a position weight matrix (PWM). The region $\pm 100 \mathrm{bp}$ of the summit of each binding peak was searched for a match of this PWM using the matchPWM function from the Biostrings v2.38.4 package in R, using a minimum motif score of $85 \%$. Whenever a binding peak had more than one motif match, the highest score was assigned to this binding peak. For all aggregate plots, the data was aligned to the motif with the highest motif score with all motifs in the same orientation as shown in Fig 5C. DNA shape analysis was done on the aligned motifs \pm 40 bp from the motif midpoint using DNAshapeR v1.10.0 (Chiu et al, 2016). The DNA shape of the bound and unbound sites from (Rossi et al, 2018a) was calculated as described in (Rossi et al, 2018a), by taking for each of the 8 motifs the top 50 and bottom 50 bound 
peaks and showing the average of the top 400 and bottom 400 bound peaks. For further details see (Rossi et al, 2018a).

\section{Statistical analysis and data visualization}

All statistical analyses were done using the statistical language R v3.2.2 except for the DNA shape and Venn diagram analyses which were done using $\mathrm{R}$ v3.5.1. The area-proportional Venn diagrams were created using the eulerr package v6.0.0 in $R$ v3.5.1.

To visualize the binding to different genomic loci in Fig 1B, the Sushi package v1.24.0 was used (Phanstiel et al, 2014). All boxplots were created using R's built-in boxplot function, with default settings; here the solid horizontal line represent the median, the box shows the interquartile range and the whiskers are at the most extreme data point no further away from the closest quartile than 1.5 times the interquartile range. Differences between the four residence time quartiles were assessed using a oneway ANOVA followed by Tukey's honest significant difference test (Fig 1J, 4B, 5B and 5D). The difference between the two groups in Fig $4 \mathrm{C}$ was tested using a twotailed t-test and between the groups in Fig 5A using a Wilcoxon rank-sum test, since one of the groups was deemed to deviate too much from normality.

\section{Data availability}

All sequencing data is available on GEO through the accession number GSE151693.

\section{Author contributions}

Conceptualization, W.d.J., P.K. and F.H.; Investigation, W.d.J. and M.B.; Formal Analysis, W.d.J., and P.L.; Software, P.L.; Data curation, W.d.J.; Supervision, P.K. and F.H.; Writing - Original Draft, W.d.J. and F.H.; Writing - Review \& Editing, W.d.J., M.B., P.L., P.K. and F.H.; Funding Acquisition, P.K. and F.H.

\section{Acknowledgements}

We thank the members of the Holstege and Kemmeren groups for their support and discussions. We thank Jeff DeMartino and Marit de Kort for technical assistance and the Utrecht sequencing facility for sequencing. This work was supported by the Netherlands Organisation for Scientific Research (NWO) grant 86411010 and by the European Research Council (ERC) grant 671174 DynaMech. 


\section{References}

Adams CC, Workman JL (1995) Binding of disparate transcriptional activators to nucleosomal DNA is inherently cooperative. Mol Cell Biol 15: 1405-1421

van Bakel H, Tsui K, Gebbia M, Mnaimneh S, Hughes TR, Nislow C (2013) A compendium of nucleosome and transcript profiles reveals determinants of chromatin architecture and transcription. PLoS Genet 9: e1003479

Bayle JH, Grimley JS, Stankunas K, Gestwicki JE, Wandless TJ, Crabtree GR (2006) Rapamycin Analogs with Differential Binding Specificity Permit Orthogonal Control of Protein Activity. Chem Biol 13: 99-107

Boer CG de, Hughes TR (2011) YeTFaSCo: a database of evaluated yeast transcription factor sequence specificities. Nucleic Acids Res: gkr993

Bosisio D, Marazzi I, Agresti A, Shimizu N, Bianchi ME, Natoli G (2006) A hyper-dynamic equilibrium between promoter-bound and nucleoplasmic dimers controls NF- $\mathrm{kB}$ dependent gene activity. EMBO J 25: 798-810

Brahma S, Henikoff S (2020) Epigenome Regulation by Dynamic Nucleosome Unwrapping. Trends Biochem Sci 45: 13-26

Brignall R, Moody AT, Mathew S, Gaudet S (2019) Considering Abundance, Affinity, and Binding Site Availability in the NF-кB Target Selection Puzzle. Front Immunol 10: 609

Buchman AR, Kornberg RD (1990) A yeast ARS-binding protein activates transcription synergistically in combination with other weak activating factors. Mol Cell Biol 10: 887-897

Busch A, Kiel T, Hübner S (2009) Quantification of Nuclear Protein Transport using Induced Heterodimerization. Traffic 10: 1221-1227

Candelli T, Challal D, Briand J-B, Boulay J, Porrua O, Colin J, Libri D (2018) High-resolution transcription maps reveal the widespread impact of roadblock termination in yeast. EMBO J 37: e97490

Cherry JM, Hong EL, Amundsen C, Balakrishnan R, Binkley G, Chan ET, Christie KR, Costanzo MC, Dwight SS, Engel SR, Fisk DG, Hirschman JE, Hitz BC, Karra K, Krieger CJ, Miyasato SR, Nash RS, Park J, Skrzypek MS, Simison M, et al (2012) Saccharomyces Genome Database: the genomics resource of budding yeast. Nucleic Acids Res 40: D700-705

Chiu T-P, Comoglio F, Zhou T, Yang L, Paro R, Rohs R (2016) DNAshapeR: an R/Bioconductor package for DNA shape prediction and feature encoding. Bioinformatics 32: 1211-1213

Coleman RA, Liu Z, Darzacq X, Tjian R, Singer RH, Lionnet T (2015) Imaging Transcription: Past, Present, and Future. Cold Spring Harb Symp Quant Biol 80: 1-8

Colin J, Candelli T, Porrua O, Boulay J, Zhu C, Lacroute F, Steinmetz LM, Libri D (2014) Roadblock Termination by Reb1p Restricts Cryptic and Readthrough Transcription. Mol Cell 56: 667-680

Collas P (2010) The current state of chromatin immunoprecipitation. Mol Biotechnol 45: 87-100

Cramer P (2019) Organization and regulation of gene transcription. Nature 573: 45-54

Dion MF, Kaplan T, Kim M, Buratowski S, Friedman N, Rando OJ (2007) Dynamics of replication-independent histone turnover in budding yeast. Science 315: 1405-1408

Dölken L, Ruzsics Z, Rädle B, Friedel CC, Zimmer R, Mages J, Hoffmann R, Dickinson P, Forster T, Ghazal P, Koszinowski UH (2008) High-resolution gene expression profiling for simultaneous kinetic parameter analysis of RNA synthesis and decay. RNA 14: 1959-1972

Donovan BT, Chen H, Jipa C, Bai L, Poirier MG (2019a) Dissociation rate compensation mechanism for budding yeast pioneer transcription factors. eLife 8:

Donovan BT, Huynh A, Ball DA, Patel HP, Poirier MG, Larson DR, Ferguson ML, Lenstra TL (2019b) Live-cell imaging reveals the interplay between transcription factors, nucleosomes, and bursting. EMBO J 38: e100809

Elbi C, Walker DA, Romero G, Sullivan WP, Toft DO, Hager GL, DeFranco DB (2004) Molecular chaperones function as steroid receptor nuclear mobility factors. Proc Natl Acad 
Sci 101: 2876-2881

Elf J, Barkefors I (2019) Single-Molecule Kinetics in Living Cells. Annu Rev Biochem 88: 635-659

Enomoto S, Longtine MS, Berman J (1994) Enhancement of Telomere-Plasmid Segregation by the X-Telomere Associated Sequence in Saccharomyces Cerevisiae Involves Sir2, Sir3, Sir4 and Abf1. Genetics 136: 757-767

Friedman N, Rando OJ (2015) Epigenomics and the structure of the living genome. Genome Res 25: 1482-1490

Furey TS (2012) ChIP-seq and beyond: new and improved methodologies to detect and characterize protein-DNA interactions. Nat Rev Genet 13: 840-852

Gailus-Durner V, Xie J, Chintamaneni C, Vershon AK (1996) Participation of the yeast activator Abf1 in meiosis-specific expression of the HOP1 gene. Mol Cell Biol 16: 2777-2786

Ganapathi M, Palumbo MJ, Ansari SA, He Q, Tsui K, Nislow C, Morse RH (2011) Extensive role of the general regulatory factors, Abf1 and Rap1, in determining genome-wide chromatin structure in budding yeast. Nucleic Acids Res 39: 2032-2044

Garbett KA, Tripathi MK, Cencki B, Layer JH, Weil PA (2007) Yeast TFIID Serves as a Coactivator for Rap1p by Direct Protein-Protein Interaction. Mol Cell Biol 27: 297-311

Gilmour DS, Lis JT (1984) Detecting protein-DNA interactions in vivo: distribution of RNA polymerase on specific bacterial genes. Proc Natl Acad Sci U S A 81: 4275-4279

Grimaldi Y, Ferrari P, Strubin M (2014) Independent RNA polymerase II preinitiation complex dynamics and nucleosome turnover at promoter sites in vivo. Genome Res 24: 117-124

Hager GL, McNally JG, Misteli T (2009) Transcription dynamics. Mol Cell 35: 741-753

Hahn S, Young ET (2011) Transcriptional regulation in Saccharomyces cerevisiae: transcription factor regulation and function, mechanisms of initiation, and roles of activators and coactivators. Genetics 189: 705-736

Halfter H, Kavety B, Vandekerckhove J, Kiefer F, Gallwitz D (1989) Sequence, expression and mutational analysis of BAF1, a transcriptional activator and ARS1-binding protein of the yeast Saccharomyces cerevisiae. EMBO J 8: 4265-4272

Hammar P, Walldén M, Fange D, Persson F, Baltekin O, Ullman G, Leroy P, Elf J (2014) Direct measurement of transcription factor dissociation excludes a simple operator occupancy model for gene regulation. Nat Genet 46: 405-408

Hartley PD, Madhani HD (2009) Mechanisms that specify promoter nucleosome location and identity. Cell 137: 445-458

Haruki H, Nishikawa J, Laemmli UK (2008) The anchor-away technique: rapid, conditional establishment of yeast mutant phenotypes. Mol Cell 31: 925-932

Hasegawa Y, Struhl K (2019) Promoter-specific dynamics of TATA-binding protein association with the human genome. Genome Res 29: 1939-1950

Ho B, Baryshnikova A, Brown GW (2018) Unification of Protein Abundance Datasets Yields a Quantitative Saccharomyces cerevisiae Proteome. Cell Syst 6: 192-205.e3

Hoopes BC, LeBlanc JF, Hawley DK (1992) Kinetic analysis of yeast TFIID-TATA box complex formation suggests a multi-step pathway. J Biol Chem 267: 11539-11547

de Jonge WJ, Brok M, Kemmeren P, Holstege FCP (2019) An extensively optimized chromatin immunoprecipitation protocol for quantitatively comparable and robust results. bioRxiv: 835926

de Jonge WJ, Brok M, Kemmeren P, Holstege FCP (2020) An Optimized Chromatin Immunoprecipitation Protocol for Quantification of Protein-DNA Interactions. STAR Protoc Available at: https://star-protocols.cell.com/protocols/71

de Jonge WJ, O'Duibhir E, Lijnzaad P, van Leenen D, Groot Koerkamp MJ, Kemmeren P, Holstege FC (2017) Molecular mechanisms that distinguish TFIID housekeeping from regulatable SAGA promoters. EMBO J 36: 274-290

Karpova TS, Chen TY, Sprague BL, McNally JG (2004) Dynamic interactions of a transcription factor with DNA are accelerated by a chromatin remodeller. EMBO Rep 5: 1064-1070 
Karpova TS, Kim MJ, Spriet C, Nalley K, Stasevich TJ, Kherrouche Z, Heliot L, McNally JG (2008) Concurrent Fast and Slow Cycling of a Transcriptional Activator at an Endogenous Promoter. Science 319: 466-469

Kasinathan S, Orsi GA, Zentner GE, Ahmad K, Henikoff S (2014) High-resolution mapping of transcription factor binding sites on native chromatin. Nat Methods 11: 203-209

Kim D, Langmead B, Salzberg SL (2015) HISAT: a fast spliced aligner with low memory requirements. Nat Methods 12: 357-360

Kivioja T, Vähärautio A, Karlsson K, Bonke M, Enge M, Linnarsson S, Taipale J (2012) Counting absolute numbers of molecules using unique molecular identifiers. Nat Methods 9: 72-74

Klemm JD, Beals CR, Crabtree GR (1997) Rapid targeting of nuclear proteins to the cytoplasm. Curr Biol 7: 638-644

Kloster-Landsberg M, Herbomel G, Wang I, Derouard J, Vourc'h C, Usson Y, Souchier C, Delon A (2012) Cellular response to heat shock studied by multiconfocal fluorescence correlation spectroscopy. Biophys J 103: 1110-1119

Krietenstein N, Wal M, Watanabe S, Park B, Peterson CL, Pugh BF, Korber P (2016) Genomic Nucleosome Organization Reconstituted with Pure Proteins. Cell 167: 709-721.e12

Kubik S, Bruzzone MJ, Jacquet P, Falcone J-L, Rougemont J, Shore D (2015) Nucleosome Stability Distinguishes Two Different Promoter Types at All Protein-Coding Genes in Yeast. Mol Cell 60: 422-434

Kubik S, Bruzzone MJ, Shore D (2017) Establishing nucleosome architecture and stability at promoters: Roles of pioneer transcription factors and the RSC chromatin remodeler. BioEssays 39: 1600237

Kubik S, O’Duibhir E, de Jonge WJ, Mattarocci S, Albert B, Falcone J-L, Bruzzone MJ, Holstege FCP, Shore D (2018) Sequence-Directed Action of RSC Remodeler and General Regulatory Factors Modulates +1 Nucleosome Position to Facilitate Transcription. Mol Cell 71: 89-102. e5

Kuo MH, Allis CD (1999) In vivo cross-linking and immunoprecipitation for studying dynamic Protein:DNA associations in a chromatin environment. Methods San Diego Calif 19: $425-433$

de Laat W, Dekker J (2012) 3C-based technologies to study the shape of the genome. Methods 58: 189-191

Lai WKM, Pugh BF (2017) Understanding nucleosome dynamics and their links to gene expression and DNA replication. Nat Rev Mol Cell Biol 18: 548-562

Larson DR (2011) What do expression dynamics tell us about the mechanism of transcription? Curr Opin Genet Dev 21: 591-599

Lascaris RF, Groot E de, Hoen P-B, Mager WH, Planta RJ (2000) Different roles for Abf1p and a T-rich promoter element in nucleosome organization of the yeast RPS28A gene. Nucleic Acids Res 28: 1390-1396

Levo M, Avnit-Sagi T, Lotan-Pompan M, Kalma Y, Weinberger A, Yakhini Z, Segal E (2017) Systematic Investigation of Transcription Factor Activity in the Context of Chromatin Using Massively Parallel Binding and Expression Assays. Mol Cell 65: 604-617.e6

Liao Y, Smyth GK, Shi W (2014) featureCounts: an efficient general purpose program for assigning sequence reads to genomic features. Bioinforma Oxf Engl 30: 923-930

Lickwar CR, Mueller F, Hanlon SE, McNally JG, Lieb JD (2012) Genome-wide protein-DNA binding dynamics suggest a molecular clutch for transcription factor function. Nature 484: 251-255

Love MI, Huber W, Anders S (2014) Moderated estimation of fold change and dispersion for RNA-seq data with DESeq2. Genome Biol 15: 550

Luo Y, North JA, Rose SD, Poirier MG (2014) Nucleosomes accelerate transcription factor dissociation. Nucleic Acids Res 42: 3017-3027

MacIsaac KD, Wang T, Gordon DB, Gifford DK, Stormo GD, Fraenkel E (2006) An improved 
map of conserved regulatory sites for Saccharomyces cerevisiae. BMC Bioinformatics 7: 113

Marahrens Y, Stillman B (1992) A yeast chromosomal origin of DNA replication defined by multiple functional elements. Science 255: 817-823

McNally JG, Müller WG, Walker D, Wolford R, Hager GL (2000) The Glucocorticoid Receptor: Rapid Exchange with Regulatory Sites in Living Cells. Science 287: 1262-1265

Miller JA, Widom J (2003) Collaborative Competition Mechanism for Gene Activation In Vivo. Mol Cell Biol 23: 1623-1632

Mirny LA (2010) Nucleosome-mediated cooperativity between transcription factors. Proc Natl Acad Sci 107: 22534-22539

Mivelaz M, Cao A-M, Kubik S, Zencir S, Hovius R, Boichenko I, Stachowicz AM, Kurat CF, Shore D, Fierz B (2020) Chromatin Fiber Invasion and Nucleosome Displacement by the Rap1 Transcription Factor. Mol Cell 77: 488-500.e9

Miyake T, Loch CM, Li R (2002) Identification of a Multifunctional Domain in Autonomously Replicating Sequence-Binding Factor 1 Required for Transcriptional Activation, DNA Replication, and Gene Silencing. Mol Cell Biol 22: 505-516

Miyake T, Reese J, Loch CM, Auble DT, Li R (2004) Genome-wide analysis of ARS (autonomously replicating sequence) binding factor 1 (Abf1p)-mediated transcriptional regulation in Saccharomyces cerevisiae. J Biol Chem 279: 34865-34872

Mueller F, Stasevich TJ, Mazza D, McNally JG (2013) Quantifying transcription factor kinetics: At work or at play? Crit Rev Biochem Mol Biol 48: 492-514

Park D, Lee Y, Bhupindersingh G, Iyer VR (2013) Widespread misinterpretable ChIP-seq bias in yeast. PloS One 8: e83506

Park PJ (2009) ChIP-seq: advantages and challenges of a maturing technology. Nat Rev Genet 10: 669-680

Paul E, Tirosh I, Lai W, Buck MJ, Palumbo MJ, Morse RH (2015) Chromatin Mediation of a Transcriptional Memory Effect in Yeast. G3 Genes Genomes Genet 5: 829-838

Perlmann T, Eriksson P, Wrange O (1990) Quantitative analysis of the glucocorticoid receptorDNA interaction at the mouse mammary tumor virus glucocorticoid response element. $J$ Biol Chem 265: 17222-17229

Phanstiel DH, Boyle AP, Araya CL, Snyder MP (2014) Sushi.R: flexible, quantitative and integrative genomic visualizations for publication-quality multi-panel figures. Bioinformatics 30: 2808-2810

Polach KJ, Widom J (1996) A Model for the Cooperative Binding of Eukaryotic Regulatory Proteins to Nucleosomal Target Sites. J Mol Biol 258: 800-812

Poorey K, Viswanathan R, Carver MN, Karpova TS, Cirimotich SM, McNally JG, Bekiranov S, Auble DT (2013) Measuring Chromatin Interaction Dynamics on the Second Time Scale at Single-Copy Genes. Science 342: 369-372

Pryde FE, Louis EJ (1999) Limitations of silencing at native yeast telomeres. EMBO J 18: 25382550

Quinlan AR, Hall IM (2010) BEDTools: a flexible suite of utilities for comparing genomic features. Bioinforma Oxf Engl 26: 841-842

Reed SH, Akiyama M, Stillman B, Friedberg EC (1999) Yeast autonomously replicating sequence binding factor is involved in nucleotide excision repair. Genes Dev 13: 3052-3058

Rhode PR, Sweder KS, Oegema KF, Campbell JL (1989) The gene encoding ARS-binding factor I is essential for the viability of yeast. Genes Dev 3: 1926-1939

Rossi MJ, Lai WKM, Pugh BF (2018a) Genome-wide determinants of sequence-specific DNA binding of general regulatory factors. Genome Res 28: 497-508

Rossi MJ, Lai WKM, Pugh BF (2018b) Simplified ChIP-exo assays. Nat Commun 9: 1-13

Roy K, Chanfreau GF (2018) A global function for transcription factors in assisting RNA polymerase II termination. Transcription 9: 41-46

Roy K, Gabunilas J, Gillespie A, Ngo D, Chanfreau GF (2016) Common genomic elements 
promote transcriptional and DNA replication roadblocks. Genome Res 26: 1363-1375

Rufiange A, Jacques P-É, Bhat W, Robert F, Nourani A (2007) Genome-Wide ReplicationIndependent Histone H3 Exchange Occurs Predominantly at Promoters and Implicates H3 K56 Acetylation and Asf1. Mol Cell 27: 393-405

Schaughency P, Merran J, Corden JL (2014) Genome-wide mapping of yeast RNA polymerase II termination. PLoS Genet 10: e1004632

Schermer UJ, Korber P, Hörz W (2005) Histones Are Incorporated in trans during Reassembly of the Yeast PHO5 Promoter. Mol Cell 19: 279-285

Schroeder SC, Weil PA (1998) Genetic Tests of the Role of Abf1p in Driving Transcription of the Yeast TATA Box Bindng Protein-encoding Gene,SPT15. J Biol Chem 273: 19884-19891

Skene PJ, Henikoff S (2017) An efficient targeted nuclease strategy for high-resolution mapping of DNA binding sites. eLife 6: e21856

Southern JA, Young DF, Heaney F, Baumgärtner WK, Randall RE (1991) Identification of an epitope on the $\mathrm{P}$ and $\mathrm{V}$ proteins of simian virus 5 that distinguishes between two isolates with different biological characteristics. J Gen Virol 72: 1551-1557

Spitz F, Furlong EEM (2012) Transcription factors: from enhancer binding to developmental control. Nat Rev Genet 13: 613-626

Stavreva DA, Müller WG, Hager GL, Smith CL, McNally JG (2004) Rapid glucocorticoid receptor exchange at a promoter is coupled to transcription and regulated by chaperones and proteasomes. Mol Cell Biol 24: 2682-2697

Struhl K, Segal E (2013) Determinants of nucleosome positioning. Nat Struct Mol Biol 20: 267273

Sun M, Schwalb B, Schulz D, Pirkl N, Etzold S, Larivière L, Maier KC, Seizl M, Tresch A, Cramer P (2012) Comparative dynamic transcriptome analysis (cDTA) reveals mutual feedback between mRNA synthesis and degradation. Genome Res 22: 1350-1359

Teytelman L, Thurtle DM, Rine J, van Oudenaarden A (2013) Highly expressed loci are vulnerable to misleading ChIP localization of multiple unrelated proteins. Proc Natl Acad Sci U S A 110: 18602-18607

Venditti P, Costanzo G, Negri R, Camilloni G (1994) ABFI contributes to the chromatin organization of Saccharomyces cerevisiae ARS1 B-domain. Biochim Biophys Acta BBA Gene Struct Expr 1219: 677-689

Voss TC, Hager GL (2014) Dynamic regulation of transcriptional states by chromatin and transcription factors. Nat Rev Genet 15: 69-81

Warner JR (1999) The economics of ribosome biosynthesis in yeast. Trends Biochem Sci 24: 437-440

van Werven FJ, van Teeffelen HAAM, Holstege FCP, Timmers HTM (2009) Distinct promoter dynamics of the basal transcription factor TBP across the yeast genome. Nat Struct Mol Biol 16: 1043-1048

Woo H, Ha SD, Lee SB, Buratowski S, Kim T (2017) Modulation of gene expression dynamics by co-transcriptional histone methylations. Exp Mol Med 49: e326-e326

Yao J, Munson KM, Webb WW, Lis JT (2006) Dynamics of heat shock factor association with native gene loci in living cells. Nature 442: 1050-1053

Yarragudi A, Miyake T, Li R, Morse RH (2004) Comparison of ABF1 and RAP1 in chromatin opening and transactivator potentiation in the budding yeast Saccharomyces cerevisiae. Mol Cell Biol 24: 9152-9164

Yarragudi A, Parfrey LW, Morse RH (2007) Genome-wide analysis of transcriptional dependence and probable target sites for Abf1 and Rap1 in Saccharomyces cerevisiae. Nucleic Acids Res 35: 193-202

Zaidi H, Hoffman EA, Shetty SJ, Bekiranov S, Auble DT (2017) Second-generation method for analysis of chromatin binding with formaldehyde-cross-linking kinetics. J Biol Chem 292: 19338-19355 


\section{Chapter 5}

Zaret KS, Carroll JS (2011) Pioneer transcription factors: establishing competence for gene expression. Genes Dev 25: 2227-2241

Zentner GE, Kasinathan S, Xin B, Rohs R, Henikoff S (2015) ChEC-seq kinetics discriminates transcription factor binding sites by DNA sequence and shape in vivo. Nat Commun 6: 8733

Zhang Y, Liu T, Meyer CA, Eeckhoute J, Johnson DS, Bernstein BE, Nusbaum C, Myers RM, Brown M, Li W, Liu XS (2008) Model-based analysis of ChIP-Seq (MACS). Genome Biol 9: R137 


\section{Supplemental Figures}

A

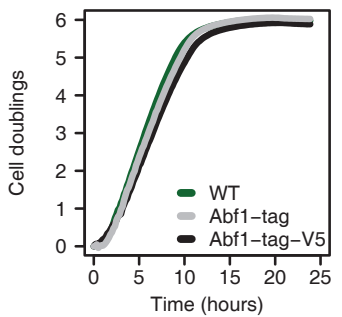

D

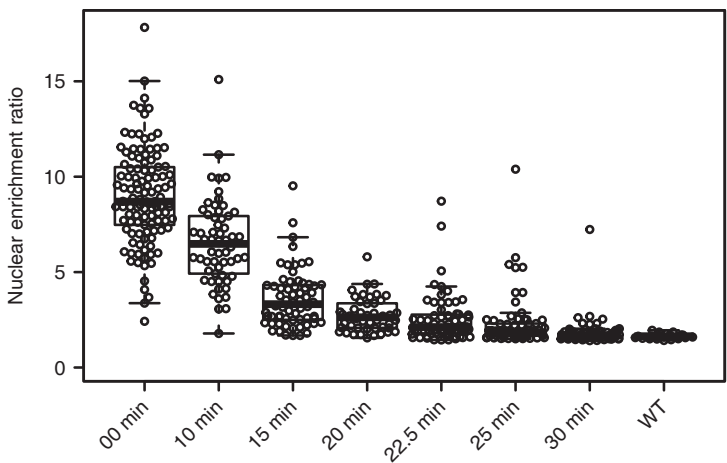

Supplemental Figure S1 Dynamics of Abf1 depletion

(A) Representative growth curves of the untagged parental background strain (WT), an ABF1 FRB-GFP-tagged strain (Abf1-tag) and an ABF1 FRBGFP-V5-tagged strain (Abf1-tag-V5). The WT strain is the parental BY4742/ S288C strain of the Abf1 anchor-away strains that have been genetically desensitized to rapamycin. The Abf1-tag strain grows approximately $10 \%$ slower compared to the WT strain and the Abf1-tag-V5 strain grows approximately $15 \%$ slower than the WT strain. (B) Growth curves of the WT strain with rapamycin, and the ABF1 FRB-GFP tagged strain with and without rapamycin, the inducing agent for nuclear depletion (Haruki et $a l, 2008)$. Rapamycin was added 4.5 hours after the start of the experiment to induce nuclear depletion (arrow). Upon induction of depletion, clear disruption of growth is visible after approximately 3 hours $(t=7.5 \mathrm{~h})$ and cessation of growth is visible after approximately 8 hours $(t=12.5 \mathrm{~h})$. (C) Representative fluorescence microscopy images of ABF1 FRB-GFP-V5 cells before ( 0 minutes) and at several time points after $(10,15,20,22.5$ and 25 minutes) induction of nuclear depletion. Scale bar: $10 \mu \mathrm{m}$. (D) Quantification of nuclear enrichment ratios of ABF1 FRB-GFP and ABF1 FRB-GFP-V5 cells during depletion (three biological replicates). Both DNA-bound and free Abf1 contribute to the GFP signal and therefore the decrease in fluorescence represents the depletion speed of both unbound as well as bound. Dots represent individual cell measurements. The nuclear enrichment ratio was calculated as the maximum intensity divided by the median intensity of each cell. A WT strain without GFP was taken along as a control.
C

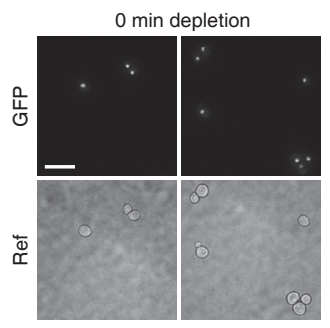

10 min depletion

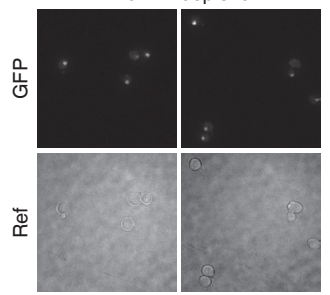

15 min depletion

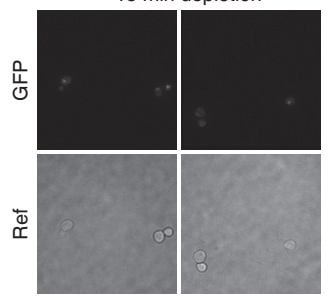

20 min depletion

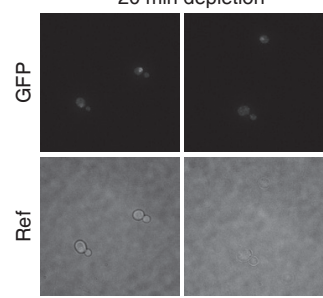

25 min depletion

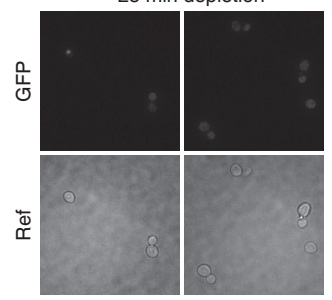




\section{Chapter 5}

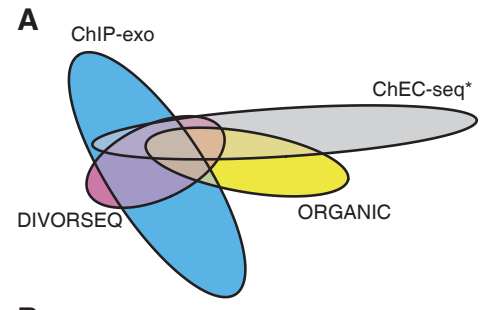

B

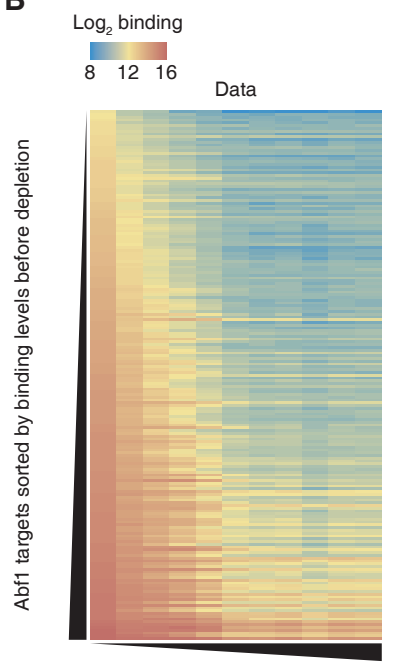

C
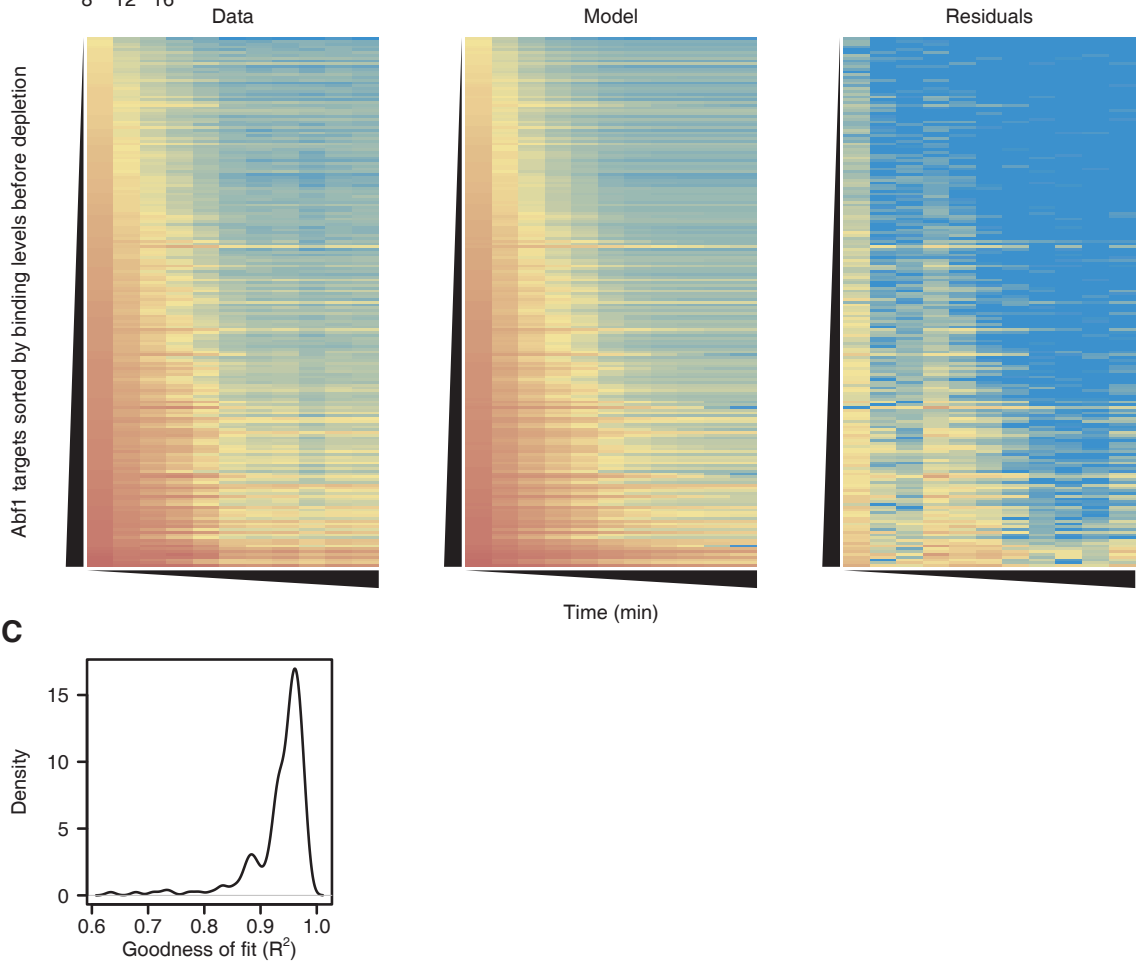

\section{Supplemental Figure S2 The exponential decay model closely fits the binding data}

(A) Venn diagram showing the overlap between all unfiltered Abf1 binding sites detected here ( $n=948)$ and published Abf1 binding sites detected using ORGANIC ChIP (Kasinathan et al, 2014; $n=1068$ ), binding sites detected using ChEC-seq that were annotated both as "fast" and "high scoring" (Zentner et al, 2015; $n=1583$ ) and binding sites that were detected using ChIP-exo v5 (Rossi et al, 2018b; $n=3177)$. (B) Heatmap representation of the average Abf1 binding at the 191 binding sites, quantified as the $\log _{2}$ number of reads in the peak (peak summit $\pm 50 \mathrm{bp}$ ). The rows are sorted on the binding levels before depletion, and the columns represent increasing time of depletion. From left to right: average binding data, fit and residuals of the fit (median absolute deviation) are shown. (C) The distribution of the goodness of fit of the 191 Abf1 binding sites. 
A

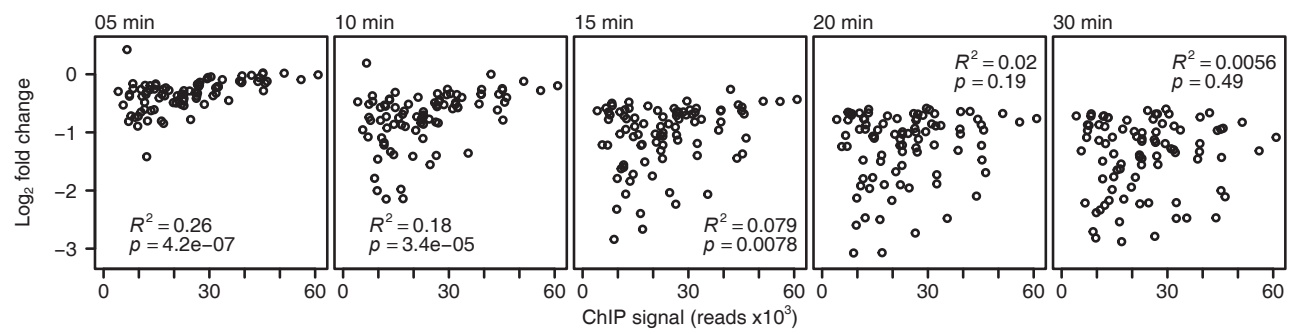

B

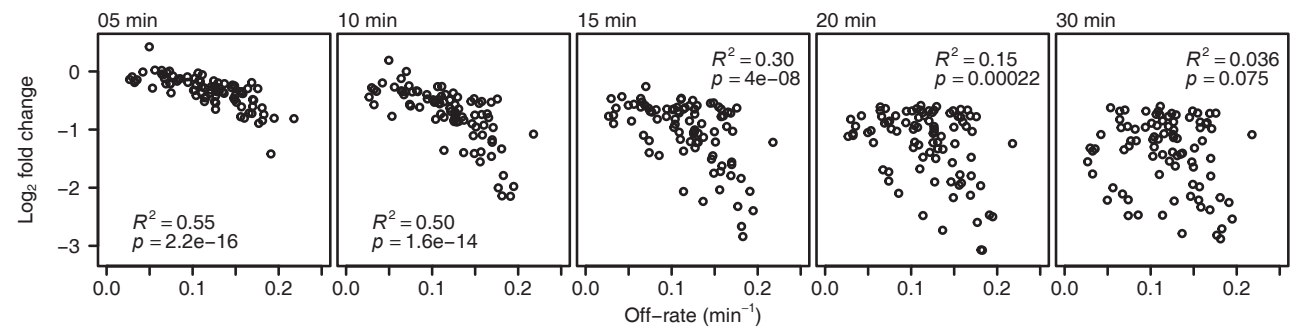

Supplemental Figure S3 Changes in mRNA synthesis correspond more closely to off-rates than binding levels

(A) Change in mRNA synthesis rate versus Abf1 binding level before depletion of the corresponding binding site after 5, 10, 15, 20 and 30 minutes of depletion of downregulated genes (fold change $>1.5$ and $p<0.01$ at 20 and 30 minutes of depletion) with Abf1 binding in the promoter $(n=88)$. The panel for $t=10$ min is also shown in Fig 3E. (B) Change in mRNA synthesis rate versus the off-rates of the corresponding binding sites for the same gene-binding sites pairs as in (A) 5, $10,15,20$ and 30 minutes after induction of depletion. The panel for $\mathrm{t}=10 \mathrm{~min}$ is also shown in Fig 3F. 



\section{Chapter 6}

\section{General Discussion}

Wim J. de Jonge, Patrick Kemmeren and Frank C.P. Holstege

Princess Máxima Center for Pediatric Oncology, Heidelberglaan 25, 3584 CS Utrecht, the Netherlands 
The work described in this thesis focuses on the dynamics of transcription regulation. The importance of this is highlighted in chapter 2 by our mechanistical analyses of the different dynamics observed between "housekeeping" genes and regulatable genes. We go on to develop methodology to determine genome-wide off-rates in vivo for the first time (chapters 3-5) and apply this to demonstrate how different TF binding dynamics can shape different aspects of TF function (chapter 5 ). Here I discuss these results, focusing on aspects not presented in the chapters themselves, especially regarding differences and commonalities in regulatability, further improvements of the discussed techniques as well as directions for future research.

\section{Global and specific requirements of SAGA and TFIID}

In chapter 2, differences in responsiveness between regulatable and housekeeping genes were investigated. To discriminate between these two gene classes, promoter binding enrichment or the lack thereof for the TFIID subunit Taf1 was used (Rhee \& Pugh, 2012). In apparent contradiction to using this approach, recent reports conclude that almost all yeast genes are dependent on both SAGA and TFIID for their transcription (Baptista et al, 2017; Warfield et al, 2017). This seems interesting in light of earlier reports which showed that a small subset of genes are more dependent on SAGA for their transcription, while the majority of genes are more dependent on TFIID (Lee et al, 2000; Huisinga \& Pugh, 2004; Rhee \& Pugh, 2012). It is important to point out that one of the main conclusions of the most highly cited study that reports differences in dependency on SAGA or TFIID is in fact: "TFIID and SAGA each contribute to the expression of nearly all genes" (Huisinga \& Pugh, 2004). This is therefore completely in line with the recent studies (Baptista et al, 2017; Warfield et al, 2017). This apparent contradiction has likely arisen because the description "dominated by SAGA or TFIID" (Huisinga \& Pugh, 2004) has been interpreted as exclusive regulation by one of these factors (Taatjes, 2017). Thus, most literature agrees that both SAGA and TFIID contribute to regulating expression of nearly all genes and therefore both are generally required for transcription.

The main disagreement between these studies is whether genes can be categorized based on a stronger dependence on either SAGA or TFIID, or not. An argument against the presence of this dichotomy is the similar binding levels of both SAGA and TFIID at promoters of genes belonging to these different classes (Grünberg et al, 2016; Baptista et al, 2017; Warfield et al, 2017). Equal binding levels of TFIID were found between SAGA- and TFIID-dominated genes when applying ChEC-seq to Taf1, the largest subunit of TFIID (Grünberg et al, 2016). This is in contrast to earlier ChIP-exo data of Taf1 (Rhee \& Pugh, 2012), which defined Taf1-enriched and depleted classes based on binding levels. This suggests that TFIID does not have a preference for binding either class. Differences between these studies may be caused by differences in cross-linking efficiency for these gene classes. Interestingly, quantified binding levels of SAGA subunits using ChEC-seq showed that SAGA binding levels at TATA-containing genes were more than twice as strong when compared to TATA-less genes (Baptista et al, 2017). This could argue for a stronger role of SAGA in regulating TATA-containing genes. However, higher binding levels were attributed to higher average expression levels of SAGA-dominated genes, 
and therefore it was concluded that SAGA regulates both classes of genes. These results show that more genes are bound by both SAGA and TFIID than previously appreciated. In addition, the binding data support a role for both SAGA and TFIID in regulating a large part of the genes in the genome. Nevertheless, it is important to point out that binding does not necessarily reflect function.

Compared to binding, a much better readout for dependency is differences in expression between SAGA- and TFIID-dominated genes upon depleting these factors. Two groups studied the effects of nuclear TFIID depletion by measuring Pol II binding using ChIP (Warfield et al, 2017; Petrenko et al, 2019). One study showed little difference in Pol II ChIP decrease between SAGA- and TFIID-dominated genes (Warfield et al, 2017), while the other found a stronger effect on TFIID-dominated genes (Petrenko et al, 2019). Interestingly, the first study also measured mRNA synthesis rates upon depletion of the TFIID subunit Taf4. When measuring mRNA production rather than Pol II binding they did observe a stronger decrease in mRNA synthesis of TFIID-dominated genes compared to SAGA-dominated genes (Warfield et al, 2017). This suggests that indeed almost all genes are affected upon depletion of TFIID, but a small subset, the SAGA-dominated genes, are less dependent on TFIID. This was confirmed by a recent and more extensive study of mRNA synthesis rates upon nuclear TFIID depletion from the same group (Donczew et al, 2020). These results confirm the notion that even though TFIID can regulate transcription of nearly all genes, some genes are less dependent on TFIID than others.

Dependence on SAGA was originally defined using total mRNA levels of individual SAGA subunit deletion strains (Huisinga \& Pugh, 2004). However, total mRNA levels after gene deletion are not necessarily a good measure to determine transcriptional dependence. In yeast, a global decrease in transcription can be buffered by globally decreasing mRNA degradation rates (Helenius et al, 2011; Sun et al, 2012; Schulz et al, 2014; Baptista et al, 2017; Timmers \& Tora, 2018). Measuring nascent mRNA levels showed that upon deletion of SAGA subunits, newly synthesized mRNA levels of both SAGA- and TFIID-dominated genes were similarly affected (Bonnet et al, 2014; Baptista et al, 2017). This shows that SAGA contributes to transcription regulation of both gene classes. Unfortunately all these deletion strains have a growth defect and slow growth itself is known to strongly affect expression levels of many genes in a completely indirect manner (O'Duibhir et al, 2014). To circumvent growth effects, nascent mRNA levels were also measured for six SAGA-dominated and six TFIIDdominated genes after induced depletion of one of the SAGA subunits (Baptista et al, 2017). Both gene classes showed similar decrease in mRNA synthesis. However, a more thorough genome-wide analysis of mRNA synthesis rates after independent depletion of several SAGA subunits showed the opposite. Instead of all genes, only a subset of genes was affected in newly synthesized mRNA levels upon SAGA subunit depletion (Donczew et al, 2020). This last study provides an explanation for these seemingly conflicting results. TFIID depletion affects most genes, but a small subset of genes $(\sim 15 \%)$ is also affected by SAGA depletion. Genes that are affected by depletion of both can use either SAGA or TFIID to bring TBP to their promoters. The authors therefore named these genes "co-activator redundant" (Donczew et 
al, 2020). The majority of genes ( $85 \%)$ only use TFIID to bring TBP to promoters and therefore these genes are TFIID-dependent. However, besides involvement in TBP recruitment, SAGA also acts as a histone acetyltransferase and a histone deubiquitinase (Rodríguez-Navarro, 2009). These other activities act on a slower timescale and are not immediately affected upon depletion of SAGA subunits. In deletion strains of SAGA subunits, all genes show altered histone modification patterns (Bonnet et al, 2014). These differences in modifications are likely the reason SAGA affects transcription of all genes in deletion strains. To summarize, most genes are likely regulated by SAGA through deposition and removal of chromatin marks, but only a subset of genes is likely dependent on SAGA for TBP recruitment.

An alternative hypothesis has been proposed to explain why some genes are more dependent on TFIID and others on SAGA. TFIID makes contacts with DNA downstream of the TATA-element in vitro and is able to support several rounds of transcription in nuclear extracts because these contacts mediate reinitiation (Joo et al, 2017). These downstream TFIID-DNA contacts were found in vivo as well. The authors propose a model whereby both SAGA and TFIID are needed for de novo assembly of the PIC, but TFIID can also facilitate reinitiation. Genes may differ in their ability to reinitiate and therefore may be dependent on SAGA and TFIID to varying degrees. Genes that cannot efficiently reinitiate need to reassemble the PIC for each round of transcription and would therefore be more dependent on SAGA. This also suggests that SAGA-dependent genes are more reliant on constant activator presence. This dependence would make them more responsive to activator depletion, which fits well with the results from chapter 2.

Despite apparent controversy, all studies support regulation of almost all yeast genes by both SAGA and TFIID. The most recent results support a scenario where the main difference between the gene classes is whether SAGA contributes to bringing TBP to the promoter or not (Donczew et al, 2020). There are now different classifications of promoters, SAGA- or TFIID-dominated (Huisinga \& Pugh, 2004), Taf1-depleted or Taf1-enriched (Rhee \& Pugh, 2012), TATA-containing or TATA-less (Basehoar et al, 2004; Rhee \& Pugh, 2012) and TFIID-dependent or coactivator-redundant (Donczew et al, 2020). All these classifications overlap with each other, but none are identical. Whether one of these classifications is better than others to classify regulatability may not be that important. A clear dichotomy may not exist and may only be observed due to our need to ascribe distinct, preferably exclusionary, categories to biological systems. This is often done even if the process being studied cannot necessarily be divided in two groups. Not all regulatable genes have a canonical TATA-box or "closed promoter" and not all housekeeping genes have a TATA-like element or are dependent on TFIID. Each gene is a mix of features and the interplay of many factors determine how regulatable a gene is, or whether it is more dependent on SAGA or TFIID. Regulatable and housekeeping classifications are likely two ends of a spectrum, with many genes residing somewhere in-between. Importantly, genes might not always be similarly dependent on either mechanism. For example, TFIID dependence was shown to vary depending on the growth medium (Warfield et al, 2017). This shows that the system is highly dynamic, and emphasizes that differences 
between studies can be caused by variations in experimental set-up rather than intrinsic differences of the processes that are being studied.

\section{The +1 nucleosome as a gatekeeper for transcription}

The +1 nucleosome has an important function for regulating transcription, since it covers the transcription start site and can control access of TBP to the promoter (Kubik et al, 2018). The +1 nucleosome may have different roles in regulating housekeeping versus regulatable genes. Despite the fact that housekeeping and regulatable genes might not be two completely distinct categories, it is currently the best way to describe differences in levels of gene regulation. For the purpose of describing the role of the +1 nucleosome in gene regulation, TFIID-dominated genes are considered housekeeping genes and SAGA-dominated genes are considered to be regulatable genes. The results in chapter 2 as well those of other studies (Nocetti \& Whitehouse, 2016; Zhou et al, 2016) showed that for regulatable genes, gene activity is tightly coupled to +1 nucleosome repositioning. The SWI/SNF complex can be recruited to promoters by GSTFs (Neely et al, 1999; Yudkovsky et al, 1999) and dynamic repositioning of the +1 nucleosome of regulatable genes was shown to be dependent on SWI/SNF (Nocetti \& Whitehouse, 2016). In contrast, the +1 nucleosome position for housekeeping genes showed little repositioning of the +1 upon activation or repression. This indicates that the "default" +1 nucleosome position for housekeeping genes is amenable to transcription, whereas for regulatable genes it is more repressive. The +1 nucleosome at regulatable genes therefore needs the activity of GSTFs and chromatin remodelers to be repositioned to allow these genes to be efficiently transcribed.

What determines this "default", more amenable for transcription positioning for housekeeping genes? These genes are enriched for poly(dA:dT) tracts (Cairns, 2009; Rando \& Winston, 2012), which are more rigid and wrap around nucleosomes less efficiently. Housekeeping promoters are therefore often considered to intrinsically disfavor nucleosome formation and consequently to be inherently more open. Studies using purified DNA and recombinant histones showed that nucleosomes can be positioned by DNA sequence alone, and NFRs can be recreated in vitro to some extent (Kaplan et al, 2009; Zhang et al, 2009, 2011; Krietenstein et al, 2016). This suggests that DNA inherently encodes nucleosome positioning. However, in vitro positioning was very weak and only became apparent when averaging the nucleosome positioning across many genes. More importantly, nucleosome positioning in vivo is constantly disturbed by the action of chromatin remodelers. Intrinsic nucleosome positioning is therefore unlikely to be the main driving force of +1 nucleosome positions in vivo. Indeed, in vitro reconstitution supplemented with chromatin remodelers recreates in vivo nucleosome positioning much more accurately (Zhang et al, 2011; Krietenstein et al, 2016). Poly(dA:dT) tracts also activate and guide RSC, which controls the NFR size of thousands of genes in the yeast genome (Krietenstein et al, 2016; Kubik et al, 2018). Positioning of the +1 nucleosome for housekeeping genes is therefore also regulated through the action of chromatin remodelers. RSC pushes the +1 and -1 nucleosome outwards, and frees up the TATA-element for efficient PIC formation (Kubik et al, 2018). Other remodelers subsequently fine-tune the exact 
nucleosome positioning (Krietenstein et al, 2016; Kubik et al, 2019). In addition to chromatin remodelers, GRFs also occupy a large fraction of promoters and can act independently of RSC to repel nucleosomes (Kubik et al, 2018). Therefore, GRFs play an important role in positioning of +1 nucleosomes as well. Global regulation of +1 nucleosome positioning indicates that proper positioning of the +1 nucleosome is needed for transcription of housekeeping genes as well. This was indeed shown by analyzing ribosomal protein genes, where gene inactivation is also associated with a shift of the +1 nucleosome covering the TATA-element. In conclusion, these studies, together with our results, are consistent with a model where the +1 nucleosome is an important determinant in transcription regulation and PIC formation for the majority of the genes in genome. The positioning of the +1 nucleosome is regulated globally by GRFs and RSC, likely through the action of RSC activating sequences (Krietenstein et al, 2016; Kubik et al, 2018, 2019). For a subset of regulatable genes, +1 nucleosome positioning is regulated by GSTFs, possibly through direct recruitment of the SWI/SNF complex (Nocetti \& Whitehouse, 2016).

A potentially interesting topic for future research could be investigating repressive and activating nucleosome positioning genome-wide, by determining the exact positioning of activating and repressive chromatin marks. If repressive chromatin marks would indeed be representative of repressive nucleosome positioning, this could be used to enrich and investigate repressive nucleosome positions for housekeeping genes. Alternatively, it would be interesting to measure the exact position of the +1 nucleosome and the activity of the corresponding gene at the same time in vivo at single cell resolution. There are technologies to track gene activity in vivo in single cells (Coulon et al, 2014; Lenstra et al, 2015). However, technologies for real-time tracking of nucleosome positioning in vivo at single cell resolution are currently lacking. If this would be possible in the future, a good model to study the interplay between nucleosome positioning and expression would be the SSA1 gene. $S S A 1$ is highly expressed and has two very clear and distinct preferred nucleosome positions that likely represent an active and inactive state (chapter 2, Fig 7). Studying nucleosome positioning and gene activity real-time in vivo will show the interplay of dynamic nucleosome positioning and transcription regulation.

\section{Further improvements for ChIP protocols}

In chapter 3, improvements to a general ChIP protocol are described. This was an important requirement for applying DIVORSEQ, since binding levels at different time points during depletion need to be quantitatively comparable to each other. However, this ChIP protocol will not be ideal for all proteins. ChIP is a multi-day protocol with many steps and differences at any one stage potentially affecting subsequent steps. For example, the extent of cross-linking affects shearing efficiency, which may in turn affect protein stability, and all three have distinct effects on quantification of the ChIP signal. Therefore, optimization of one step will affect the others, which subsequently may need to be reconsidered as well. Some proteins may not cross-link efficiently and may need longer cross-linking times. Alternatively, some proteins may differ in cross-linking efficiency between binding sites. Abf1 for example is only efficiently cross-linked to sites with either guanidine or cytosine residue at $-8 \mathrm{bp}$ from 
its binding motif (Rossi et al, 2018), allowing accurate off-rate determination only at these sites (chapter 5). Some proteins are more sensitive to protein degradation, making them more sensitive to variation in how consistently the protocol is executed. Particularly strong binders may need very stringent washes to prevent the protein of interest from associating with DNA during the immunoprecipitation step. This was for example observed for Abf1. Low levels of depleted and non-cross-linked Abf1 could rebind to some loci during the immunoprecipitation step (chapter 5), which made some sites to appear not to deplete at all. More stringent washes could have removed these binding events. However, very stringent washes may also disrupt weak antibody-antigen binding. The read-out used for quantification of binding is important as well. Conditions that are ideal for ChIP-qPCR might not necessarily be optimal for ChIP-seq. These differences are likely the reason why there are so many variations of the ChIP protocol in use across the research landscape. Each has been optimized using different proteins and equipment. Even though the ChIP protocol that was optimized in chapter 3 may not be ideal for studying binding of each individual protein, the steps that introduce the most variation are highlighted in this chapter. This knowledge should help to guide optimizations of the protocol for other proteins as well. Ideally, this protocol will be optimized again for any novel protein studied.

Besides protein-specific optimization and even though many steps of the ChIP protocol were extensively optimized (chapter 3), there are further general improvements to be made. One improvement that could significantly benefit the quantification of binding levels is normalizing to a positive control rather than a negative signal such as background. Spike-ins or external controls have been in use for a long time for expression analyses (Holstege et al, 1998; van de Peppel et al, 2003; Sun et al, 2012) and spike-ins are now occasionally used as well for ChIP (Bonhoure et al, 2014; Orlando et al, 2014) or MNase-seq (Chereji et al, 2019). In fact, there has been a growing realization that spike-ins are important for all genome-wide analyses (Chen et al, 2016). There are several ways spike-ins can be used. Concomitantly, how and when spike-ins are added during the procedure should be carefully considered. All spike-ins for ChIP use material from an evolutionarily distant species, to be able to accurately distinguish whether DNA fragments come from the sample or from the spike-ins. The easiest way to add spike-ins is by spiking in chromatin at the immunoprecipitation step, to correct for differences in immunoprecipitation efficiency. This only works well if the same antibody can be used to detect both the protein of interest as well as the spiked-in protein. This approach works well for histones that are highly conserved between species and can be recognized by the same antibody. Otherwise, the same affinity tag should be used for both the protein of interest and the spike-in control. Still, this does not prevent other discrepancies during the procedure, such as lysis or shearing efficiency. Therefore, the ideal spikein is adding cells with a similar physiology as the cells of interest before cell lysis. For example, in the case of performing ChIP with Saccharomyces cerevisiae, the spikein cells should also have a cell wall, such as Schizosaccharomyces pombe. It is important to note that this assumes differences in lysis efficiency between samples are identical for these cell types, which may not be the case. It also assumes that differences in 
protein stability are the same between the protein of interest and the tagged spike-in control protein. Based on the results in chapter 3, this assumption may not always hold, since some proteins are more likely to be degraded during the ChIP protocol. Therefore, ideally, a protein that is homologous to the protein of interest is used as a spike-in control, tagged with the same affinity tag. If this is not possible, a protein with a similar mass as the protein of interest should be used, since large proteins are more prone to degradation. In summary, even though spike-ins can improve the normalization of binding levels, the exact strategy used should be carefully considered.

\section{Further improvements for DIVORSEQ}

DIVORSEQ in its current form can be used to determine genome-wide off-rates of proteins and link binding dynamics to their function. Nevertheless, as a method it may still be improved. A limitation of DIVORSEQ is that, at least for Abf1, the lowest resolution is a residence time of a few minutes. This is due to the high abundance of Abf1 ( 6,000 molecules) and the depletion speed of the anchor away system $(\sim 2,000$ molecules per minute). For less abundant proteins DIVORSEQ will be able to measure shorter residence times. To improve the lower detection limit, a few improvements can be made. For example, if depletion speed is limiting, a second export system may be used to deplete the protein of interest from the nucleus more rapidly. The anchoraway system has a single ribosomal protein fused to FKBP12 to deplete proteins from the nucleus upon addition of rapamycin (Haruki et al, 2008). An additional export system could involve FKBP12 fused to a second ribosomal protein to double the depletion speed or fused to a nuclear export signal (NES), which has also been used to deplete proteins from the nucleus (Geda et al, 2008; Patury et al, 2009). By combining anchor-away with a second ribosomal anchor, NES-tagged depletion, or both, quicker nuclear depletion may be achieved. More rapid depletion will be especially beneficial for abundant proteins. Besides faster depletion, several other adaptations could also improve the procedure. To increase temporal resolution, more frequent sampling of binding is likely needed. Potentially, more efficient crosslinking, such as UV cross-linking, could also increase signal for proteins that do not efficiently cross-link using formaldehyde. In this case, the ChIP protocol must be adjusted since UV cross-links are difficult to reverse and may damage DNA (Zhang et al, 2004). Finally, the current ChIP protocol cannot distinguish between binding sites that are close together. By applying DIVORSEQ with a higher-resolution ChIP protocol, for example ChIP-exo (Rhee \& Pugh, 2011) or fragmentation using MNase (Skene \& Henikoff, 2015; Arrigoni et al, 2018; Gutin et al, 2018), it should be possible to determine differences in off-rates between closely spaced sites.

Another potential improvement for DIVORSEQ is using ChEC-seq or CUT\&RUN rather than ChIP to measure binding levels. These techniques do not suffer from the same limitations as ChIP. However, there are drawbacks which may make them less than ideal, especially with ChEC-seq. Using ChEC-seq for DIVORSEQ means that along with the FRB-tag, MNase must also be fused to the protein of interest, making the tag substantially larger. This may disrupt protein function, which should therefore be assessed first. ChEC-seq and CUT\&RUN do not use 
cross-linking, which may not be compatible with DIVORSEQ. Cross-linking allows for "snapshots" of binding levels at different time points during the depletion time course, by preventing dissociation of the TF of interest. Without cross-linking, the protein of interest may dissociate and bind to other genomic locations. ChEC-seq is not compatible with cross-linking since MNase will be inactivated. Therefore, DIVORSEQ should first be compared with and without cross-linking to assess whether omission of cross-linking is possible. In contrast to ChEC-seq, CUT\&RUN can detect binding using cross-linked material (Skene \& Henikoff, 2017). CUT\&RUN is more sensitive than ChIP. Applying DIVORSEQ with CUT\&RUN rather than ChIP could therefore improve sensitivity. With more sensitive detection of binding levels, off-rates of sites with low binding levels could potentially be determined as well.

\section{Abf1 likely binds to telomeres in a stable fashion}

With an improved DIVORSEQ protocol it would be interesting to investigate Abf1 binding stability at telomeres in more detail. Abf1 binds to telomeres and is involved in subtelomeric silencing (Pryde \& Louis, 1999). Due to stringent peak filtering for sites with inefficient cross-linking, off-rates were only obtained for $4 / 28$ telomeres with detectable Abf1 binding. Binding of Abf1 to telomeres is very stable, with $3 / 4$ telomeres in the top $15 \%$ of longest residence times (Fig 1A). Abf1 binding at telomeres showed intriguing characteristics that differ from non-telomeric binding sites. For example, the summit of the average Abf1 ChIP signal for non-telomeric binding sites is located -7 bp upstream from the binding motif (Fig 1B and 1C). This is consistent with cross-linking close to this site (Rossi et al, 2018). In contrast, the binding summit of telomeric sites is located on average $20 \mathrm{bp}$ more downstream, at +13 bp (Fig 1B and 1C). This suggests that Abf1 is cross-linked in a different manner at telomeric versus non-telomeric binding sites, suggesting that Abf1 makes different DNA contacts at telomeres. In addition, telomeres have a very uniform and distinct nucleosome organization, centered on Abf1 binding (Fig 1D and 1E). The nucleosome that is present on the $3^{\prime}$ end of the Abf1 binding site is located just downstream of the Abf1 binding motif (Fig 1D and 1E). Abf1 may therefore contact the nucleosome or even be bound to a small stretch of nucleosomal DNA. Depletion of Abf1 leads to a decreased occupancy of this 3' nucleosome (Fig 1E, dashed line), which suggests that Abf1 and this nucleosome may bind cooperatively. Strikingly, the one telomere with a very distinct nucleosome organization compared to other telomeres (Fig 1D, arrow) also has a much higher Abf1 off-rate compared to the other telomeric sites (Fig 1A, arrow). This suggests that either nucleosome architecture at telomeres facilitates very stable Abf1 binding, or that stable Abf1 binding is needed to create this distinct pattern of nucleosome positioning.

It is currently unknown what causes differences in binding stability between nontelomeric and telomeric binding of Abf1. Nevertheless, there are two hypotheses that both involve cooperative binding which could explain this stable binding. It is important to note however that at this stage both theories are highly speculative. The first hypothesis involves cooperative binding with Rad proteins. Abf1 is also known to be involved in nucleotide excision repair and cofractionates with Rad7 and Rad16 (Reed et al, 1999; van Eijk et al, 2019). Phosphorylation of serine 129 of 
A

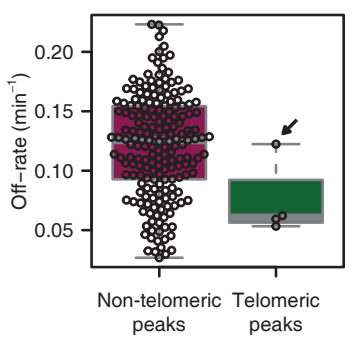

D

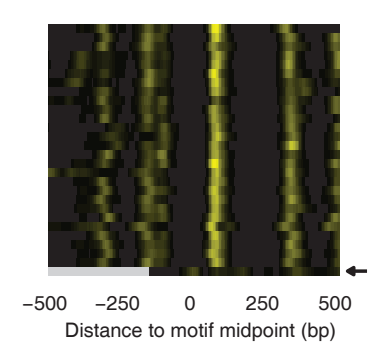

B

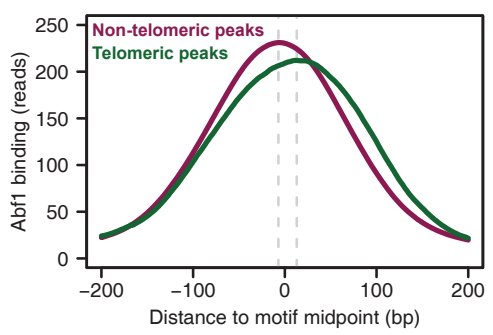

E

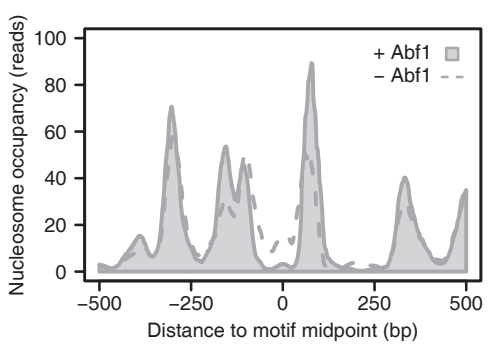

C

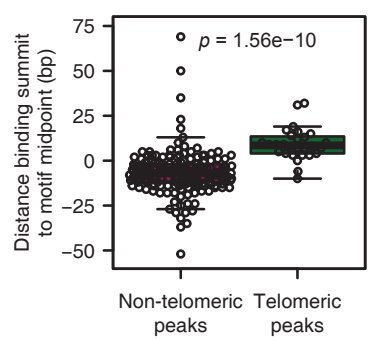

Figure 1 Abf1 stably binds to telomeres

(A) Comparison of off-rates between telomeric and non-telomeric sites. (B) Distance of the motif midpoint to the ChIP-seq peak summit of telomeric and non-telomeric peaks. (C) Average ChIP-seq binding signal of non-telomeric Abf1 peaks and all 28 telomeric peaks centered on the Abf1 binding motif. (D) Nucleosome binding at all 28 Abf1 bound telomeres, centered on the Abf1 binding motif. The data was oriented such that each Abf1 binding motif has the same orientation and the end of the chromosome is on the left. Nucleosome positioning data is from chapter 2. Gray denotes areas without binding data. (E) Average nucleosome binding at all 28 Abf1-bound telomeres, before (light gray fill) and after (dashed line) Abf1 depletion. Nucleosome positioning data is from (Kubik et al, 2015), and is reoriented and centered as in (D). The arrow in (A) and (D) marks the one telomere that has a twofold higher off-rate of Abf1 compared to the other telomeric sites.

histone 2A (H2AS129ph) is a known mark of DNA damage (also known as $\gamma$-H2AX) and this mark is highly enriched in telomeres (Kim et al, 2007; Szilard et al, 2010; Weiner et al, 2015). Perhaps Abf1 binds to telomeres together with Rad7 and Rad16, and this association can stabilize Abf1 binding. As an alternative hypothesis to Rad proteins, Sir proteins may be involved. Sir proteins are highly enriched at telomeres. The peak of Sir protein binding coincides with Abf1 binding, and Abf1 has been suggested to recruit Sir proteins to telomeres (Ellahi et al, 2015). Sir proteins can interact with histones, and perhaps simultaneous interactions of Sir proteins with Abf1 and histones can stabilize Abf1 binding. This would imply cooperative binding of Abf1 with nucleosomes and Sir proteins, and could potentially explain why Abf1 cross-links differently to telomeric sites compared to non-telomeric sites.

Unfortunately, investigating the role of Abf1 at telomeres was not possible in the scope of these studies, since most telomeric binding sites were excluded from all analyses. These sites had to be excluded because Abf1 cannot efficiently be crosslinked at these sites due to the motif sequence and therefore off-rates could 
not be determined reliably. However, more detailed analyses of exact Rad and Sir protein binding could indicate which of these mechanisms is more likely to stabilize Abf1 binding. With an improved DIVORSEQ protocol, more accurate determination of off-rates at all telomeres may be possible. Determining Abf1 off-rates after deletion or inducible depletion of either the Rad or Sir proteins, for example using a degron system (Nishimura et al, 2009), could reveal whether these proteins can stabilize Abf1 binding at telomeres.

\section{Future applications of DIVORSEQ}

So far DIVORSEQ has only been applied to a single TF, Abf1, which is an abundant protein and a stable binder. DIVORSEQ would possibly need some of the improvements suggested above to accurately determine off-rates of lowly abundant and more transiently binding TFs. An improved DIVORSEQ protocol could be applied to many other proteins, potentially in combination with cross-linking kinetic analysis to determine on-rates in parallel (Poorey et al, 2013). This should reveal how dynamics of different proteins shape their function. For example, repressors may be expected to be stably bound, and differences in binding stability may determine how quickly target genes can be activated. There may also be clear differences in binding stability between the co-activators SAGA and TFIID. TBP has different binding dynamics at different promoters depending on whether it binds in the context of SAGA or TFIID (van Werven et al, 2009). It is subsequently possible that SAGA and TFIID have different dynamics as well, which may also be expected if TFIID is a scaffold for reinitiation for certain genes (Joo et al, 2017). GTFs are expected to be very dynamic when the PIC is constantly reassembled after each initiation cycle, but PICs may be more stable for certain genes. For example, GTFs that are involved in gene looping (Singh \& Hampsey, 2007; Wang \& Roberts, 2010) may be more stably bound at promoters which loop versus promoters that do not. By studying binding dynamics of many different proteins that regulate transcription, a better picture will emerge of how binding dynamics differ between genomic locations and how binding dynamics contribute to the functions of different factors involved in transcription regulation.

\section{Binding dynamics determine transcription dynamics}

By studying binding dynamics in a systematic manner, the role of binding dynamics in transcription dynamics can be further elucidated. Since transcription is a stochastic process, involving many steps, genes are often transcribed in bursts rather than in a constitutive manner. Regulatable genes show an especially stronger bursting behavior (Lenstra et al, 2016). The dynamics of activator binding is intimately linked to dynamics and bursting of transcription (Brouwer \& Lenstra, 2019). This was shown directly for the transcription factor Gal4, where the residence time is strongly correlated to burst duration (Donovan et al, 2019). Recent developments in single cell sequencing technologies have claimed to be able to infer both burst frequency and burst size in a genome-wide manner (Larsson et al, 2019). The data suggest that burst frequency is primarily determined by enhancers and burst size by core promoters. If this technology proves to be accurate, it would be fascinating to couple genomewide bursting measurements with genome-wide binding dynamics. This could show 
whether there is indeed a clear relationship between bursting and binding dynamics for many TFs, and provide insight into how these TFs dynamically regulate their targets.

\section{Phase separation and dynamic transcriptional control}

A concept that has recently received a lot of attention with regard to transcription regulation is phase separation. Many components of the transcription machinery can phase separate, meaning that they form distinct clusters or condensates in the nucleus. This happens when concentrations are sufficiently high and there is a crowded environment, such as in the nucleus. TFs, mediator and Pol II can phase separate in vitro, but also form condensates in vivo (Banani et al, 2017; Boija et al, 2018; Sabari et al, 2018). These condensates are reminiscent of small liquid droplets, and this type of phase separation is called liquid-liquid phase separation. Phase separation provides an explanation for mechanisms that were previously hard to explain, such as interactions between TF activation domains and mediator (Boija et al, 2018). These interactions involve intrinsically disordered domains and cannot be explained by classical "lock-and-key" mechanisms. Phase separation can control several aspects of transcription. For example, depending on its phosphorylation status, Pol II phase separates with different types of condensates containing different factors involved in initiation or splicing (Guo et al, 2019). Phase separation will also affect dynamics of binding. Frequency of binding within a condensate will be higher because the on-rate is dependent on the concentration, and the local concentration is increased in condensates. Conversely, the concentration outside of the condensates is lower, meaning that frequency of binding outside these condensates will be reduced. Therefore, transcriptional bursting is likely also affected by phase separation through modulation of the on-rate (Brouwer \& Lenstra, 2019). Although phase separation studies have mainly been focused on human cells, yeast GSTFs can also form condensates (Wollman et al, 2017; Boija et al, 2018). Future studies will provide more insight whether phase separation also plays an important role in yeast transcription regulation and binding dynamics.

\section{Concluding remarks}

Transcription is crucial to life as we know it, and misregulation of transcription can have extremely detrimental effects. Transcription is a dynamic process, regulated by dynamic interactions. The results described in this thesis (chapter 2 and 5) demonstrate the importance of binding dynamics in achieving appropriate regulation. This has implications for disease too. Since binding is governed by both on- and off-rates, mutations or genomic variation that influence these rates will also result in phenotypic changes, in the worst case disease. Understanding how transcription is dynamically regulated is therefore crucial, not only to understand the molecular mechanisms of transcription, but will likely also contribute to our understanding of phenotypic variation and disease susceptibility. Future studies combining measurements of binding dynamics with transcription dynamics will greatly contribute to our understanding of how transcription is dynamically regulated in health and disease. 


\section{References}

Arrigoni L, Al-Hasani H, Ramírez F, Panzeri I, Ryan DP, Santacruz D, Kress N, Pospisilik JA, Bönisch U, Manke T (2018) RELACS nuclei barcoding enables high-throughput ChIP-seq. Commun Biol 1: 214

Banani SF, Lee HO, Hyman AA, Rosen MK (2017) Biomolecular condensates: organizers of cellular biochemistry. Nat Rev Mol Cell Biol 18: 285-298

Baptista T, Grünberg S, Minoungou N, Koster MJE, Timmers HTM, Hahn S, Devys D, Tora L (2017) SAGA Is a General Cofactor for RNA Polymerase II Transcription. Molecular Cell 68: 130-143.e5

Basehoar AD, Zanton SJ, Pugh BF (2004) Identification and distinct regulation of yeast TATA box-containing genes. Cell 116: 699-709

Boija A, Klein IA, Sabari BR, Dall'Agnese A, Coffey EL, Zamudio AV, Li CH, Shrinivas K, Manteiga JC, Hannett NM, Abraham BJ, Afeyan LK, Guo YE, Rimel JK, Fant CB, Schuijers J, Lee TI, Taatjes DJ, Young RA (2018) Transcription Factors Activate Genes through the Phase-Separation Capacity of Their Activation Domains. Cell 175: 1842-1855.e16

Bonhoure N, Bounova G, Bernasconi D, Praz V, Lammers F, Canella D, Willis IM, Herr W, Hernandez N, Delorenzi M, CycliX Consortium (2014) Quantifying ChIP-seq data: a spiking method providing an internal reference for sample-to-sample normalization. Genome Res 24: 1157-1168

Bonnet J, Wang C-Y, Baptista T, Vincent SD, Hsiao W-C, Stierle M, Kao C-F, Tora L, Devys D (2014) The SAGA coactivator complex acts on the whole transcribed genome and is required for RNA polymerase II transcription. Genes Dev 28: 1999-2012

Brouwer I, Lenstra TL (2019) Visualizing transcription: key to understanding gene expression dynamics. Current Opinion in Chemical Biology 51: 122-129

Cairns BR (2009) The logic of chromatin architecture and remodelling at promoters. Nature 461: 193-198

Chen K, Hu Z, Xia Z, Zhao D, Li W, Tyler JK (2016) The Overlooked Fact: Fundamental Need for Spike-In Control for Virtually All Genome-Wide Analyses. Mol Cell Biol 36: 662-667

Chereji RV, Bryson TD, Henikoff S (2019) Quantitative MNase-seq accurately maps nucleosome occupancy levels. Genome Biol 20: 198

Coulon A, Ferguson ML, de Turris V, Palangat M, Chow CC, Larson DR (2014) Kinetic competition during the transcription cycle results in stochastic RNA processing. eLife 3: e03939

Donczew R, Warfield L, Pacheco D, Erijman A, Hahn S (2020) Two roles for the yeast transcription coactivator SAGA and a set of genes redundantly regulated by TFIID and SAGA. eLife 9: e50109

Donovan BT, Huynh A, Ball DA, Patel HP, Poirier MG, Larson DR, Ferguson ML, Lenstra TL (2019) Live-cell imaging reveals the interplay between transcription factors, nucleosomes, and bursting. The EMBO Journal 38: e100809

van Eijk P, Nandi SP, Yu S, Bennett M, Leadbitter M, Teng Y, Reed SH (2019) Nucleosome remodeling at origins of global genome-nucleotide excision repair occurs at the boundaries of higher-order chromatin structure. Genome Res 29: 74-84

Ellahi A, Thurtle DM, Rine J (2015) The Chromatin and Transcriptional Landscape of Native Saccharomyces cerevisiae Telomeres and Subtelomeric Domains. Genetics 200: 505-521

Geda P, Patury S, Ma J, Bharucha N, Dobry CJ, Lawson SK, Gestwicki JE, Kumar A (2008) A small molecule-directed approach to control protein localization and function. Yeast 25: 577-594

Grünberg S, Henikoff S, Hahn S, Zentner GE (2016) Mediator binding to UASs is broadly uncoupled from transcription and cooperative with TFIID recruitment to promoters. The EMBO Journal: e201695020 
Guo YE, Manteiga JC, Henninger JE, Sabari BR, Dall'Agnese A, Hannett NM, Spille J-H, Afeyan LK, Zamudio AV, Shrinivas K, Abraham BJ, Boija A, Decker T-M, Rimel JK, Fant CB, Lee TI, Cisse II, Sharp PA, Taatjes DJ, Young RA (2019) Pol II phosphorylation regulates a switch between transcriptional and splicing condensates. Nature 572: 543-548

Gutin J, Sadeh R, Bodenheimer N, Joseph-Strauss D, Klein-Brill A, Alajem A, Ram O, Friedman N (2018) Fine-Resolution Mapping of TF Binding and Chromatin Interactions. Cell Reports 22: $2797-2807$

Haruki H, Nishikawa J, Laemmli UK (2008) The anchor-away technique: rapid, conditional establishment of yeast mutant phenotypes. Mol Cell 31: 925-932

Helenius K, Yang Y, Tselykh TV, Pessa HKJ, Frilander MJ, Mäkelä TP (2011) Requirement of TFIIH kinase subunit Mat1 for RNA Pol II C-terminal domain Ser5 phosphorylation, transcription and mRNA turnover. Nucleic Acids Res 39: 5025-5035

Holstege FCP, Jennings EG, Wyrick JJ, Lee TI, Hengartner CJ, Green MR, Golub TR, Lander ES, Young RA (1998) Dissecting the Regulatory Circuitry of a Eukaryotic Genome. Cell 95: $717-728$

Huisinga KL, Pugh BF (2004) A genome-wide housekeeping role for TFIID and a highly regulated stress-related role for SAGA in Saccharomyces cerevisiae. Mol Cell 13: 573-585

Joo YJ, Ficarro SB, Soares LM, Chun Y, Marto JA, Buratowski S (2017) Downstream promoter interactions of TFIID TAFs facilitate transcription reinitiation. Genes Dev 31: 2162-2174

Kaplan N, Moore IK, Fondufe-Mittendorf Y, Gossett AJ, Tillo D, Field Y, LeProust EM, Hughes TR, Lieb JD, Widom J, Segal E (2009) The DNA-encoded nucleosome organization of a eukaryotic genome. Nature 458: 362-366

Kim J-A, Kruhlak M, Dotiwala F, Nussenzweig A, Haber JE (2007) Heterochromatin is refractory to $\gamma$-H2AX modification in yeast and mammals. J Cell Biol 178: 209-218

Krietenstein N, Wal M, Watanabe S, Park B, Peterson CL, Pugh BF, Korber P (2016) Genomic Nucleosome Organization Reconstituted with Pure Proteins. Cell 167: 709-721.e12

Kubik S, Bruzzone MJ, Challal D, Dreos R, Mattarocci S, Bucher P, Libri D, Shore D (2019) Opposing chromatin remodelers control transcription initiation frequency and start site selection. Nat Struct Mol Biol 26: 744-754

Kubik S, Bruzzone MJ, Jacquet P, Falcone J-L, Rougemont J, Shore D (2015) Nucleosome Stability Distinguishes Two Different Promoter Types at All Protein-Coding Genes in Yeast. Molecular Cell 60: 422-434

Kubik S, O'Duibhir E, de Jonge WJ, Mattarocci S, Albert B, Falcone J-L, Bruzzone MJ, Holstege FCP, Shore D (2018) Sequence-Directed Action of RSC Remodeler and General Regulatory Factors Modulates +1 Nucleosome Position to Facilitate Transcription. Mol Cell 71: 89-102. e5

Larsson AJM, Johnsson P, Hagemann-Jensen M, Hartmanis L, Faridani OR, Reinius B, Segerstolpe Å, Rivera CM, Ren B, Sandberg R (2019) Genomic encoding of transcriptional burst kinetics. Nature 565: 251

Lee TI, Causton HC, Holstege FCP, Shen W-C, Hannett N, Jennings EG, Winston F, Green MR, Young RA (2000) Redundant roles for the TFIID and SAGA complexes in global transcription. Nature 405: 701-704

Lenstra TL, Coulon A, Chow CC, Larson DR (2015) Single-Molecule Imaging Reveals a Switch between Spurious and Functional ncRNA Transcription. Molecular Cell 60: 597-610

Lenstra TL, Rodriguez J, Chen H, Larson DR (2016) Transcription Dynamics in Living Cells. Annu Rev Biophys 45: 25-47

Neely KE, Hassan AH, Wallberg AE, Steger DJ, Cairns BR, Wright APH, Workman JL (1999) Activation Domain-Mediated Targeting of the SWI/SNF Complex to Promoters Stimulates Transcription from Nucleosome Arrays. Molecular Cell 4: 649-655

Nishimura K, Fukagawa T, Takisawa H, Kakimoto T, Kanemaki M (2009) An auxin-based degron system for the rapid depletion of proteins in nonplant cells. Nat Methods 6: 917-922 
Nocetti N, Whitehouse I (2016) Nucleosome repositioning underlies dynamic gene expression. Genes Dev 30: 660-672

O'Duibhir E, Lijnzaad P, Benschop JJ, Lenstra TL, van Leenen D, Groot Koerkamp MJ, Margaritis T, Brok MO, Kemmeren P, Holstege FC (2014) Cell cycle population effects in perturbation studies. Mol Syst Biol 10: 732

Orlando DA, Chen MW, Brown VE, Solanki S, Choi YJ, Olson ER, Fritz CC, Bradner JE, Guenther MG (2014) Quantitative ChIP-Seq Normalization Reveals Global Modulation of the Epigenome. Cell Reports 9: 1163-1170

Patury S, Geda P, Dobry CJ, Kumar A, Gestwicki JE (2009) Conditional nuclear import and export of yeast proteins using a chemical inducer of dimerization. Cell Biochem Biophys 53: 127-134

van de Peppel J, Kemmeren P, van Bakel H, Radonjic M, van Leenen D, Holstege FCP (2003) Monitoring global messenger RNA changes in externally controlled microarray experiments. EMBO Rep 4: 387-393

Petrenko N, Jin Y, Dong L, Wong KH, Struhl K (2019) Requirements for RNA polymerase II preinitiation complex formation in vivo. eLife 8: e43654

Poorey K, Viswanathan R, Carver MN, Karpova TS, Cirimotich SM, McNally JG, Bekiranov S, Auble DT (2013) Measuring Chromatin Interaction Dynamics on the Second Time Scale at Single-Copy Genes. Science 342: 369-372

Pryde FE, Louis EJ (1999) Limitations of silencing at native yeast telomeres. The EMBO Journal 18: $2538-2550$

Rando OJ, Winston F (2012) Chromatin and transcription in yeast. Genetics 190: 351-387

Reed SH, Akiyama M, Stillman B, Friedberg EC (1999) Yeast autonomously replicating sequence binding factor is involved in nucleotide excision repair. Genes Dev 13: 3052-3058

Rhee HS, Pugh BF (2011) Comprehensive genome-wide protein-DNA interactions detected at single-nucleotide resolution. Cell 147: 1408-1419

Rhee HS, Pugh BF (2012) Genome-wide structure and organization of eukaryotic pre-initiation complexes. Nature 483: 295-301

Rodríguez-Navarro S (2009) Insights into SAGA function during gene expression. EMBO Rep 10: 843-850

Rossi MJ, Lai WKM, Pugh BF (2018) Genome-wide determinants of sequence-specific DNA binding of general regulatory factors. Genome Res 28: 497-508

Sabari BR, Dall'Agnese A, Boija A, Klein IA, Coffey EL, Shrinivas K, Abraham BJ, Hannett NM, Zamudio AV, Manteiga JC, Li CH, Guo YE, Day DS, Schuijers J, Vasile E, Malik S, Hnisz D, Lee TI, Cisse II, Roeder RG, et al (2018) Coactivator condensation at super-enhancers links phase separation and gene control. Science 361: eaar3958

Schulz D, Pirkl N, Lehmann E, Cramer P (2014) Rpb4 Subunit Functions Mainly in mRNA Synthesis by RNA Polymerase II. J Biol Chem 289: 17446-17452

Singh BN, Hampsey M (2007) A transcription-independent role for TFIIB in gene looping. Mol Cell 27: 806-816

Skene PJ, Henikoff S (2015) A simple method for generating high-resolution maps of genomewide protein binding. eLife 4: e09225

Skene PJ, Henikoff S (2017) An efficient targeted nuclease strategy for high-resolution mapping of DNA binding sites. eLife 6: e21856

Sun M, Schwalb B, Schulz D, Pirkl N, Etzold S, Larivière L, Maier KC, Seizl M, Tresch A, Cramer P (2012) Comparative dynamic transcriptome analysis (cDTA) reveals mutual feedback between mRNA synthesis and degradation. Genome Res 22: 1350-1359

Szilard RK, Jacques P-É, Laramée L, Cheng B, Galicia S, Bataille AR, Yeung M, Mendez M, Bergeron M, Robert F, Durocher D (2010) Systematic identification of fragile sites via genome-wide location analysis of $\gamma$-H2AX. Nat Struct Mol Biol 17: 299-305

Taatjes DJ (2017) The Continuing SAGA of TFIID and RNA Polymerase II Transcription. 
Molecular Cell 68: 1-2

Timmers HTM, Tora L (2018) Transcript Buffering: A Balancing Act between mRNA Synthesis and mRNA Degradation. Mol Cell 72: 10-17

Wang Y, Roberts SG (2010) New insights into the role of TFIIB in transcription initiation. Transcription 1: 126-129

Warfield L, Ramachandran S, Baptista T, Devys D, Tora L, Hahn S (2017) Transcription of Nearly All Yeast RNA Polymerase II-Transcribed Genes Is Dependent on Transcription Factor TFIID. Molecular Cell 68: 118-129.e5

Weiner A, Hsieh T-HS, Appleboim A, Chen HV, Rahat A, Amit I, Rando OJ, Friedman N (2015) High-resolution chromatin dynamics during a yeast stress response. Mol Cell 58: 371-386

van Werven FJ, van Teeffelen HAAM, Holstege FCP, Timmers HTM (2009) Distinct promoter dynamics of the basal transcription factor TBP across the yeast genome. Nat Struct Mol Biol 16: 1043-1048

Wollman AJ, Shashkova S, Hedlund EG, Friemann R, Hohmann S, Leake MC (2017) Transcription factor clusters regulate genes in eukaryotic cells. eLife 6: e27451

Yudkovsky N, Logie C, Hahn S, Peterson CL (1999) Recruitment of the SWI/SNF chromatin remodeling complex by transcriptional activators. Genes Dev 13: 2369-2374

Zhang L, Zhang K, Prändl R, Schöffl F (2004) Detecting DNA-binding of proteins in vivo by UV-crosslinking and immunoprecipitation. Biochemical and Biophysical Research Communications 322: 705-711

Zhang Y, Moqtaderi Z, Rattner BP, Euskirchen G, Snyder M, Kadonaga JT, Liu XS, Struhl K (2009) Intrinsic histone-DNA interactions are not the major determinant of nucleosome positions in vivo. Nat Struct Mol Biol 16: 847-852

Zhang Z, Wippo CJ, Wal M, Ward E, Korber P, Pugh BF (2011) A packing mechanism for nucleosome organization reconstituted across a eukaryotic genome. Science 332: 977-980

Zhou X, Blocker AW, Airoldi EM, O'Shea EK (2016) A computational approach to map nucleosome positions and alternative chromatin states with base pair resolution. eLife 5: e16970 


\section{Addendum}

Nederlandse samenvatting

Author contributions per chapter

List of publications

Curriculum Vitae

Acknowledgements 


\section{Nederlandse Samenvatting}

\section{Het belang van DNA en RNA}

In al het leven dat wij kennen staat DNA centraal. Het vormt lange strengen die bestaan uit een aaneenschakeling van vier verschillende "letters", afgekort met A, C, G en T. DNA is het erfelijk materiaal dat de instructies bevat voor het maken en functioneren van alle organismen. De samenstelling van de letters in het DNA verschilt tussen organismen, maar ook enigszins tussen individuen. In het DNA staan instructies hoe cellen functioneren. DNA fungeert dus als een "kookboek" of blauwdruk van instructies voor de cel. De instructies in het DNA noemen we genen en deze zijn als het ware de recepten in een kookboek. Genen bevatten de instructies voor het maken van eiwitten en deze eiwitten zijn de machientjes in de cel die alle verschillende functies uitvoeren.

Het DNA zelf kan echter geen eiwitten maken, net zoals een kookboek geen taart kan bakken. Daarom wordt het DNA eerst gekopieerd naar een boodschappermolecuul, het zogenaamde RNA. Deze RNA moleculen worden vervolgens herkend door ribosomen. Dit zijn speciale fabriekjes die met behulp van de instructies in het RNA eiwitten maken. De eiwitten kunnen vervolgens hun functies uitoefenen in de cel.

\section{Het aflezen van DNA: transcriptie}

Alle cellen in een organisme, zoals een mens of plant, hebben allemaal hetzelfde DNA. Toch kunnen met behulp van hetzelfde DNA zeer verschillende celtypes gemaakt worden die allemaal andere functies hebben. Cellen met verschillende functies moeten verschillende eiwitten maken. Zo maken levercellen bijvoorbeeld enzymen om alcohol af te breken, maar deze eiwitten zijn niet nodig in cellen in je huid of hart. Andersom moeten cellen in het hart eiwitten maken om samen te trekken, maar deze eiwitten zijn weer niet nodig in de lever. De verschillen tussen cellen en welke eiwitten in welke celtypes aanwezig zijn, worden voor een groot gedeelte bepaald doordat het DNA op verschillende momenten en in verschillende celtypes anders wordt afgelezen. Dit proces van het aflezen van DNA heet transcriptie. Transcriptie is dus een heel belangrijk proces in de cel en het is belangrijk dat dit goed gereguleerd wordt zodat de juiste eiwitten op de juiste momenten in de juiste celtypes gemaakt worden.

De regulatie van transcriptie, dus welke stukken DNA op welk moment worden afgelezen, wordt grotendeels bepaald door het binden van zogenaamde transcriptiefactoren aan DNA. Deze transcriptiefactoren kunnen binden op specifieke plekken in het DNA en zo genen "aan" of "uit" zetten. Transcriptiefactoren binden niet op één plek in het DNA, maar op meerdere plekken. Eén transcriptiefactor reguleert dus meerdere genen tegelijk. Op deze manier bepalen transcriptiefactoren welke genen gekopieerd worden in RNA en dus welke eiwitten er gemaakt worden in een cel.

\section{Regulatie van transcriptie is dynamisch}

In dit onderzoek hebben wij de regulatie van transcriptie door transcriptiefactoren 
bestudeerd in het eencellige organisme gist. Wij hebben gist als modelsysteem gebruikt omdat het transcriptieproces van gist op fundamenteel niveau erg vergelijkbaar is met dat van de mens. Gist heeft daarbij als voordeel dat het snel groeit in het laboratorium en dat er eenvoudig DNA veranderingen aangebracht kunnen worden om zo specifieke transcriptiefactoren of specifieke stukken DNA te analyseren.

Transcriptie wordt vaak gezien als een statisch proces: een gen staat "aan" of "uit". Dit is echter niet het geval. Net zoals er in een auto een gaspedaal en een rem zit die beide belangrijk zijn voor het veilig aankomen op de bestemming, zo worden genen constant aan- en uitgezet. Hoe sterk een gen aanstaat wordt ook gereguleerd, vergelijkbaar met dat een auto harder gaat rijden wanneer het gaspedaal verder wordt ingedrukt. Transcriptie is dus op deze en andere manieren een zeer dynamisch proces.

Dat transcriptie dynamisch is, is heel belangrijk want het stelt een cel in staat zich aan te passen aan veranderende omgevingen. Zo groeit tijdens de ontwikkeling één enkele cel uit tot een volwaardig mens. In de ontwikkelende en delende cellen moeten steeds andere genen aan- en uitgezet worden. Dit is onder andere afhankelijk van wat voor cellen het zijn en waar in het lichaam ze zich bevinden. Voor een gistcel zouden deze veranderende omgevingen bijvoorbeeld hoge zoutconcentraties of hogere temperaturen kunnen zijn. Om te overleven in deze veranderende omstandigheden moet een cel zich snel aan kunnen passen en steeds genen op een dynamische manier aan- en uitzetten. Aangezien transcriptie een dynamisch proces is, moeten de factoren die dit reguleren ook dynamisch zijn. Sterker nog, de dynamiek van transcriptie wordt grotendeels bepaald door de dynamiek van de binding van transcriptiefactoren aan DNA.

In de studies die beschreven zijn in dit proefschrift hebben wij de dynamiek van transcriptie van verschillende klassen van genen onderzocht. Daarnaast hebben we een nieuwe methode ontwikkeld om de bindingsdynamiek van eiwitten aan DNA te meten. Deze methode hebben we vervolgens toegepast op een transcriptiefactor om te onderzoeken hoe de bindingsdynamiek bijdraagt aan de regulatie van transcriptie door deze transcriptiefactor.

\section{Bevindingen beschreven in dit proefschrift}

Genen worden vaak in twee klassen ingedeeld die door andere co-activatoren gereguleerd worden. Co-activatoren worden zo genoemd omdat ze bijdragen aan het transcriptieproces. In gist zijn er twee co-activatoren die verschillende groepen genen reguleren. Deze co-activatoren zijn SAGA en TFIID. Genen die belangrijk zijn voor het reageren op veranderende omstandigheden gebruiken vaak SAGA als coactivator. Genen die een meer huishoudelijke functie hebben, maken vaker gebruik van TFIID als co-activator. In hoofdstuk 2 hebben wij het verschil in dynamiek van de regulatie van deze twee verschillende klassen van genen onderzocht. Dit hebben wij gedaan door een transcriptiefactor te bestuderen die beide klassen van genen reguleert, genaamd Hsf1. Door de hoeveelheid Hsf1 dat aan DNA kan binden te 
veranderen, kunnen we zien in welke mate deze genen afhankelijk zijn van Hsf1. De twee klassen genen (SAGA en TFIID genen) reageren met een andere dynamiek op het veranderen van de hoeveelheid Hsf1 in de celkern. De SAGA genen blijken responsiever te zijn. Wij laten zien dat dit komt doordat deze genen meer afhankelijk zijn van permanente aanwezigheid van Hsf1. Deze afhankelijkheid wordt veroorzaakt doordat de SAGA genen meer negatieve regulatie hebben van andere eiwitten. Deze studie laat zien dat het dynamisch binden van een transcriptiefactor een ander effect kan hebben afhankelijk van het klasse gen dat gebonden wordt en verklaart tevens wat het achterliggende moleculaire mechanisme is.

De resultaten in hoofdstuk 2 bevestigen dat de manier waarop transcriptiefactoren genen reguleren voor een groot deel afhankelijk is van de dynamiek waarmee ze DNA binden. Er zijn echter weinig methodes om de bindingsdynamiek van een eiwit op meerdere plekken op het DNA tegelijkertijd te meten. Daarom ontwikkelden wij een nieuwe methode om de bindingsdynamiek van eiwitten op alle DNA-gebonden plekken tegelijkertijd te meten. Deze methode meet de snelheid waarmee een eiwit loslaat van het DNA, ook wel de dissociatiesnelheid of "off-rate" genoemd. Om deze reden noemen we de methode DIVORSEQ (Determining In Vivo Off-rates by Sequencing). Voor deze methode maken we gebruik van een techniek genaamd chromatine immunoprecipitatie (ChIP). Met ChIP kan de binding van een eiwit aan al het DNA tegelijkertijd bepaald worden. Hoewel ChIP al meer dan 30 jaar gebruikt wordt, zijn de meeste ChIP protocollen niet kwantitatief. Dit wil zeggen dat de protocollen wel kunnen bepalen welke stukken DNA een transcriptiefactor kan binden, maar niet "hoeveel" binding er plaatsvindt. Voor de bepaling van "offrates" is het echter cruciaal dat de bindingsmetingen wel op een kwantitatieve wijze met elkaar vergeleken kunnen worden. Daarom hebben wij eerst het ChIP protocol aangepast, zodat dit kwantitatief vergelijkbare resultaten oplevert. Dit werd bereikt door bijna alle stappen in het protocol te optimaliseren. Hoofstuk 3 beschrijft de wijze waarop het protocol geoptimaliseerd is. Dit geoptimaliseerde protocol wordt uitgebreid beschreven in hoofdstuk 4.

In hoofdstuk 5 hebben wij met behulp van de nieuwe methode om bindingsdynamiek te meten de "off-rates" voor bijna 200 van de bindingplaatsen van de transcriptiefactor Abf1 gemeten. We vonden dat verschillende bindingsplekken grote verschillen in "off-rates" hebben. Deze verschillen worden deels veroorzaakt door de specifieke volgorde van de letters van het DNA op de bindingsplek, maar ook door de vorm die het DNA op de bindingsplek aanneemt. Daarnaast speelt mogelijk competitie met andere DNA-gebonden eiwitten een rol. De resultaten laten ook zien dat de "off-rates" belangrijk zijn voor de regulatie van transcriptie, zowel bij het aanzetten van genen als het bij het stoppen van transcriptie.

Transcriptie is een dynamisch proces dat gereguleerd wordt door factoren die dynamisch DNA binden. Ondanks dat transcriptie een fundamenteel proces is voor al het leven zoals wij dat kennen, weten we nog maar relatief weinig over deze dynamiek. De resultaten in dit proefschrift dragen bij aan de kennis over de dynamiek van transcriptie regulatie. Dit is belangrijk, want door de dynamiek van 
transcriptie te bestuderen kunnen we beter begrijpen hoe transcriptie op moleculair niveau gereguleerd wordt en uiteindelijk hoe misregulatie van transcriptie kan leiden tot ziektes zoals kanker. 


\section{Author contributions per chapter}

\section{Chapter 1: General Introduction}

Writing - Original Draft, W.d.J, P.K. and F.H.; Writing - Review \& Editing, W.d.J, P.K. and F.H.

Chapter 2: Molecular mechanisms that distinguish TFIID housekeeping from regulatable SAGA promoters

Conceptualization, E.O.D., W.d.J. and F.H.; Investigation, E.O.D., W.d.J., D.v.L. and M.G.K. Formal Analysis, E.O.D., W.d.J., and P.L.; Writing, E.O.D., W.d.J. and F.H.; Supervision, P.K. and F.H.; Funding Acquisition, P.K. and F.H.

\section{Chapter 3 Extensive optimization of chromatin immunoprecipitation for} quantitatively comparable and robust results

Conceptualization, W.d.J., P.K and F.H.; Investigation, W.d.J. and M.B.; Writing Original Draft, W.d.J. and F.H; Writing - Review \& Editing, W.d.J., M.B., P.K and F.H.; Funding Acquisition, P.K and F.H.; Supervision, P.K and F.H.

Chapter 4 An optimized chromatin immunoprecipitation protocol for quantification of protein-DNA interactions

Conceptualization, W.d.J., P.K and F.H.; Investigation, W.d.J. and M.B.; Writing Original Draft, W.d.J. and F.H; Writing - Review \& Editing, W.d.J., M.B., P.K and F.H.; Funding Acquisition, P.K and F.H.; Supervision, P.K and F.H.

\section{Chapter 5 Genome-wide off-rates reveal how DNA binding dynamics shape} transcription factor function

Conceptualization, W.d.J., P.K. and F.H.; Investigation, W.d.J., and M.B.; Formal Analysis, W.d.J. and P.L.; Software, P.L.; Data curation, W.d.J.; Supervision, P.K. and F.H.; Writing - Original Draft, W.d.J. and F.H.; Writing - Review \& Editing, W.d.J., M.B., P.L., P.K. and F.H.; Funding Acquisition, P.K. and F.H.

\section{Chapter 6: General Discussion}

Writing - Original Draft, W.d.J, P.K. and F.H.; Writing - Review \& Editing, W.d.J, P.K. and F.H. 


\section{List of publications}

de Jonge WJ, Brok M, Lijnzaad P, Kemmeren P, Holstege FCP (2020) Genome-wide off-rates reveal how DNA binding dynamics shape transcription factor function. bioRxiv: 2020.05.03.074815

Submitted to Molecular Systems Biology

de Jonge WJ, Brok M, Kemmeren P, Holstege FCP (2020) An Optimized Chromatin Immunoprecipitation Protocol for Quantification of Protein-DNA Interactions. STAR Protocols: 71

de Jonge WJ, Brok M, Kemmeren P, Holstege FCP (2019) An extensively optimized chromatin immunoprecipitation protocol for quantitatively comparable and robust results. bioRxiv: 835926

Kubik S, O'Duibhir E, de Jonge WJ, Mattarocci S, Albert B, Falcone J-L, Bruzzone MJ, Holstege FCP, Shore D (2018) Sequence-Directed Action of RSC Remodeler and General Regulatory Factors Modulates +1 Nucleosome Position to Facilitate Transcription. Mol Cell 71: 89-102.e5

de Jonge WJ*, O’Duibhir $E^{*}$, Lijnzaad P, van Leenen D, Groot Koerkamp MJ, Kemmeren P, Holstege FCP (2017) Molecular mechanisms that distinguish TFIID housekeeping from regulatable SAGA promoters. EMBO J 36: 274-290

*equal contribution

Long Y, Smet W, Cruz-Ramírez A, Castelijns B, de Jonge WJ , Mähönen AP, Bouchet BP, Perez GS, Akhmanova A, Scheres B, Blilou I (2015) Arabidopsis BIRD Zinc Finger Proteins Jointly Stabilize Tissue Boundaries by Confining the Cell Fate Regulator SHORT-ROOT and Contributing to Fate Specification. Plant Cell 27: 1185-1199 


\section{Curriculum Vitae}

Wim de Jonge was born on August $20^{\text {th }} 1990$ in 's-Hertogenbosch, the Netherlands. He received his Gymnasium diploma from the Sint-Janslyceum in 2008. In 2011 he received his Bachelor's degree in Biology (cum laude) from the University of Utrecht. He then continued with the Master's program Molecular and Cellular Life Sciences also in Utrecht. During his first internship he studied protein-protein interactions of transcription factors that control root development in the plant $A$. thaliana, under the supervision of Dr. Yuchen Long and Dr. Ikram Blilou in the lab of Prof. dr. Ben Scheres at the University of Utrecht. His second internship was carried out in the department of Molecular Cancer Research in the University Medical Center Utrecht under supervision of Dr. Eoghan O'Duibhir in the lab of Prof. dr. Frank Holstege. Here he studied the molecular mechanisms underlying differences in regulation between different gene classes. In 2014 he continued this research as a PhD candidate under the supervision of Dr. Patrick Kemmeren and Prof. dr. Frank Holstege to study transcription regulation dynamics. The first two years of his $\mathrm{PhD}$ were carried out at the University Medical Center Utrecht. In 2016 the lab moved to the Princess Máxima Center for Pediatric Oncology. The lab was temporarily located at the Hubrecht Institute and in 2018 the lab relocated to the building of the Princess Máxima Center. The results of this research are described in this thesis. Wim de Jonge will join the lab of Dr. Tineke Lenstra at the Netherlands Cancer Institute in Amsterdam as a postdoctoral scientist. 


\section{Acknowledgements}

Some say that four years of $\mathrm{PhD}$ is a long time. Others would argue that a period of six years is too short, especially when these years are divided over three different institutes. I would say that a PhD is not too long as long as you are still learning new things and enjoying yourself. Six years (yes yes, a bit more than six, but for convenience I am rounding it down) and three institutes later I have made many new friends, had a great time and a lot of fun. Therefore, there are a lot of people that I would like to thank for (mental) support and fun times during my PhD.

Frank, after my internship in your group I stayed so long that you had no choice but to offer me a PhD position, and I am very grateful that you did. To me, you were a great supervisor. Your door was always open when I needed feedback, even though you were often very busy. During my PhD you had a perfect balance between giving me the freedom to plan my own experiments and showing interest in the projects without pushing too much. Throughout my PhD you have been my example of how someone should do science. You are always emphasizing the importance of being precise, as well as being critical about others and our own work and also promoting open science and willing to share data and strains with everyone. Although writing papers with you was always (somewhat) frustrating, I was always amazed how you could transform my writing into a perfectly smooth story that is much easier to read. Thank you for all the advice you gave me over the years, about science but also about life in general. Importantly, besides a great group leader you are also a very kind and social person you can laugh with outside of the lab. I enjoyed (almost) all of our meetings (including discussions about why "ORA-Seq" would not be a good name for our method or conversations about interesting carnaval costumes) and I think we get along quite well, which is part of the reason why I stayed so long. I don't think I could have wished for a better supervisor.

Patrick, my co-promotor, without you my PhD would not have been possible. Thank you for guidance and advice over the years. Even though at the end I was more part of Frank's group than yours, you were nearly always presemt at our weekly meetings to offer your views on the project as a bioinformatician. I am also very grateful for your advice and feedback especially towards the end of my PhD. I am pretty sure I would not have made the deadline of my thesis without your help.

Eoghan, you were sort of my third (perhaps technically first) PhD supervisor. I am sure that I would not have been able to start my PhD without you. I am grateful for how well you trained me when I was a master student. You showed me how to properly plan experiments, be precise, analyze big data, (ab)use published data that is readily available, to "think big" and all other skills needed to start a PhD. I really enjoyed working together on chapter 2. You were a great inspiration for how I should do my PhD. In fact, somebody once told me that I was the "Eoghan 2.0" of the lab, which I considered a great compliment. I am very happy that we have remained friends after you were finished and really enjoyed visiting you and your family in Scotland. 
A PhD cannot be concluded without a PhD- and a reading committee. Thank you Alexander van Oudenaarden and Marc Timmers for being in my PhD committee and for your advice during the PhD evaluation meetings each year. Wouter de Laat, Marc Timmers, Sander van den Heuvel, Fred van Leeuwen and Tineke Lenstra, thank you for taking the time and effort to read and assess my thesis.

Of course, I have to thank my paranymphs. Bas, ever since we were "benchneighbors" in the Kruyt building, listening to Sky radio and singing along with all songs (actually that was mostly you, but still) we have been friends. Our paths kept crossing later in the Stratenum and also during our PhD's when our lab was temporarily located at the Hubrecht. Our friendship has been shaped by our shared love for beers, borrels, carnaval, board games, pub quizzes and "gezelligheid" in general. Thanks for always being ready to drink a beer and for co-organizing the carnaval trips as well as several legendary pub quizzes. I hope that we will enjoy the fantastic BBQ's in your garden for many years to come. Jeff, we both started out as master students in our group and continued with a PhD, only I started 3 years earlier. This means that we were in the lab together for a large part of my PhD. Thanks for always being in for a cup of tea or having a little chat, for making fun of others (and each other) and outside-of-work-beers (although not as frequently as we both wanted). You are one of those people that is too kind, and always wants to help even when you actually don't have the time to do so. Thanks for all your help over the years, both in the lab as well as a critical reviewer of several of my chapters and papers. Although you were already familiar with the lab, starting as a PhD student in a lab that switched fields is tough, and the start of your PhD was not as easy as you (and everyone else) had hoped. Still, your projects are really shaping up and they will lead to nice publications for sure. If you finish before the six-year mark you will still be quicker than me!

I would really like to thank all the current and past members of the Holstege lab. For all the fun times together, the lunch breaks, amazing retreats, support and scientific input! Mariël, without you I would probably still be optimizing the ChIP protocol or growing yeast. Thanks for working on the (sometimes frustrating) projects together with me. I think we were a great team and we were very efficient while working together. The countless hours in the yeast lab were always fun. Right before and during the lab-moves you had (a little bit) less time to work on our projects, but that was clearly necessary to make the moves as smooth as possible. Still you tried to help me whenever you had time, and the help was greatly appreciated. A very big thank you to Philip for all the help and bioinformatics support (mostly in R) that you have given me during my PhD (and before that), which was crucial for my projects. I can't think of any problem that I had that you couldn't solve, or you would come up with a nifty workaround. Problems that I had been stuck with for hours and you would usually solve within minutes. Your knowledge of statistics seems endless. Actually, that also counts for your knowledge about pretty much anything that is written on Wikipedia, and you are always very happy to share that knowledge. Marian thank you for all the help, especially with the first project and all the microarrays and GEO submission that you and Dik did for me. Thanks as 
well for the occasional help with growth experiments or technical advice on my experiments. The past year has been very rough for you, but I am happy to see that you are doing much better and are eager (as you are always) to get back to work. Don't forget to take it easy! Thanasis, I hope I am not offending anyone, but you are one of the greatest scientific minds I have ever met. It amazes me every time how you always have great ideas for how things work at a mechanistic level and how you always know the exact right experiments or controls to figure out how things work. I am very grateful you were always there at the group meetings (and outside of the group meetings) to give me advice and suggestions for projects. Thanks for all the cups of tea and cookies and for being a great friend. Besides your scientific input you have a great family and you and Cornee always make feel very welcome at your home when we had dinner or played games. Thanks again for your old chairs! They are my favorite piece of furniture in the house. I am sorry that I did not have time to help you guys out in the facility. Tito, since you joined the lab I think you changed quite a bit (mostly for the better), but despite working on single cell sequencing your love for yeast-research remained. Thanks for the occasional bioinformatics support, the in-depth discussions about my project and all your insights, especially about roadblock termination. I have very fond memories of us working together on the borrels, crafting exclamation marks and making ice cream using liquid nitrogen. Both the D\&D themed and the "Icy Indulgence" borrel were legendary. Thanks as well for all your cooking advice, I owe you a lot for all these great (Italian) recipes you taught me ("it is not always about efficiency"). Most importantly, thanks for all the fun times, the borrels, Muchkin, (speed-) watching Marvel movies together and taking me to see Opeth. Thanks for being a great friend! Eduard, thanks for being a friendly desk-neighbor and for running many of our samples on the bioanalyzer for us. Thanks as well for the many hours we spend in the pre-PCR room together and sharing a similar taste of music. Metal makes everything better, especially when you have to pipette 384-well qPCRs. Don't forget to (re-)start the game evenings when it is allowed to organize them again! Michael you are the living proof that not all Germans lack a sense of humor (although this may partly be due to the fact that genetically you are only half-German). The knowledge about pediatric cancers you brought to all group meetings was always highly appreciated. During our group meetings you like to play devil's advocate, which was actually pretty nice and helped us to be critical about our projects. Thanks for also competing each day for the first-at-work trophy, without you it wouldn't have been a challenge. Lindy, my corona-times-afternoon-meeting-buddy. Thanks for our buddy calls during the lockdown and while I was writing my thesis, they were always nice breaks of the writing routine. Sorry for the many lunchbreaks when we kept rambling on about games and such for too long. Things might get better when I am gone. It is inspiring to see how quickly you have become an expert in single cell sequencing analysis and immune cells. Keep up the good work! Ewa, you started in our lab to reduce the workload in the culture lab, but unfortunately most of the burden ended up on your shoulders really quickly. It amazes me how well you handled the pressure and how hard and motivated you continued to work, as well as how eager you were to get back to the lab when it became possible again after the lockdown. Try to not work too hard. Tomasz, you are a very crucial component of the Máxima as our FACS 
operator. Although you were usually too busy to attend our group meetings, it was great having you there on our retreats. I love your enthusiasm for other people's projects and your enthusiasm for the borrels as well. The last few months were rough for you, but it is great to see that you're back.

Since the Holstege and Kemmeren group operated as a single entity for the most of my $\mathrm{PhD}$ and because I was also part of the Kemmeren group, I want to thank the members of the Kemmeren Lab. Saman, my buddy and partner-in-crime for the biggest part of my PhD. Sorry for forcing you to join all the pub quizzes and borrels, but thank you for all the times you were there, as well as for the master classes and $\mathrm{PhD}$ retreats we joined as roommates (never forget toothpaste!) and not to forget the games of foosball, futsal matches and games of table tennis we played on our makeshift table in the Hubrecht. All these times can only be described as "Very Nice!" (How much?). You were always smiling, and your smile is very contagious. Although I really missed you when you finished, I am very happy that the end of your PhD did not mean the end of our friendship. I was very honored that I could be present at your wedding and I hope that I will be able to visit you and Kim in your new house soon! Hinri, I've always very much appreciated your sense of humor (I think I am one of the few), especially when you were teaching Dutch to Saman. Thanks for making me laugh and brightening my days. Many thanks for your help whenever I ran into problems with HPC. Jayne, thank you for introducing me to vegemite with white bread as a real Australian dish, since you rightfully pointed out that every yeast researcher should try this at least once. Thanks for making me realize that I never want to eat that again. Josephine, you were for a large part the social representative at borrels for the Kemmeren group. Thanks for always making sure you could join them at least for a bit, I very much enjoyed our talks about everything and nothing at these borrels. Thanks also for organizing the bioinformatics meetings, although I usually had to skip the meeting due to time restraints, I really enjoyed the few that I joined. Ellen, thank you for organizing a great retreat together with Mariel and for the love that we share for board games. Chris, thanks for your witty remarks during meetings and for your help with moving our data and the webservers. That saved me a lot of time. Laurène thanks for our little chats during the borrels. Too bad we didn't get to say goodbye when you left due to the corona-crisis. I hope you are doing well in France.

Of course, I also have to thank all the former members of our lab. Nathalie, during my first Christmas dinner of the lab we talked about how we are both from Brabant and love to celebrate carnaval, which immediately created a bond. Thank you for keeping the lab organized in the first years of my PhD. Dik, it was very though for you when we moved to the Máxima and you had to stay at the Stratenum. I am happy that you managed to combine your passion for science and computers at the IT-department. Thank you for all the help and microarrays that you did for my project and the IT-support afterwards. Cheuk, thank you for all your support and for making many batches of medium, but also for your help even when we were not part of the UMC anymore. Thanks also for the foosball and tea breaks together with Thanasis and Saman. They were always a very welcome distraction. Sandy, 
thank you for all your help and assistance during my time in the UMC. I can assure you, everyone in the lab really missed you when we moved. I am happy you haven't forgotten us after all those years. We haven't forgotten you for sure! I hope your health and energy will improve soon, it is inspiring to see your positive attitude in spite of all the circumstances. Sander van Hooff, you finished your PhD before we moved to the Máxima but you missed us so much that you joined the Máxima last year. It is nice to see that you are doing well as head-bioinformatician in the Molenaar group. Christian, unfortunately you were only with us for a year, but we had a lot of fun together. It always cracked me up when you were reading your protocol and started with "even kijken". Thanks for always bringing "flüssiger Stickstoff" for me. I hope you are doing well in Austria. Willemijn, you were the reason our lab started working on sarcomas. Thank you for your medical input during the time you were here. Good luck with the sarcoma research in Switzerland.

I also have to thank all the master students that joined our lab during my PhD. First of all, Esther, thank you for introducing me to the Holstege group. Without you I would likely not have started here! I really enjoyed our time together as master students in the group, also with Trang and Wiebe. Sander Lambo, (aka Slambo) you joined our lab during a difficult time for you. But you did well and had very successful PhD in Heidelberg, which you finished quicker than me. Good luck with pursuing a scientific career! Jan, Philip and I supervised you during your internship here. Your project was a tough and artificial mix of lab- and bioinformatics work, which you managed to balance. I hope you are still having fun at MRC-Holland. Billie, it was very nice having you with us. Your project turned out to be a lot harder than we anticipated, but you kept trying to make everything work. Importantly, you invented the "artistic 2D-migration technique", which I will remember in case I need to make my Western blots prettier. Jurrian, already as a master student you proved to be highly talented and motivated. We were all really sad that you couldn't do your PhD in our group. I am glad that you decided to stay at the Máxima and I am sure that you will do great in the group of Ruben. Marit, as you know from your laudatio, you did really well during your time here. It was unfortunate that we didn't have time to continue with and publish your part of the project. Still, I think we had a lot of fun working on the off-rates project together, which is the most important part. I am looking forward to our time in the Lenstra lab together! Floranne, you were the last student of our group before it became too crowded in the Máxima. It was nice to see how your project evolved and you managed to detect CNVs in single cells. Good luck with your PhD in Groningen!

I would really like to thank our collaborators David Shore and Slawek Kubik. First of all, for my visit to your lab to help us with the MNase-seq experiments. Thank you also for the scientific discussions over the years and including us in your 2018 Mol Cell paper, it was inspiring to see your dedication and perseverance in getting it published. Lastly, thanks for generating many great and high quality datasets over the years, these were a great help especially for analyzing our DIVORSEQ data in chapter 5 . 
I would like to thank all the support staff that made it possible for us to do science. The first two years of my PhD our lab was located in the Stratenum building of the UMC Utrecht. Marcel, thank you for cleaning and taking care of countless of glass bottles and Erlenmeyers that we used each week when growing yeast. Marjoleine, thanks for taking care of all the ordering together with Cheuk and making sure we would have the reagents we needed for our experiments. A big thanks to the people of the IT-department: Wim, Mark, Eric, Dennis, Martin and Dik for managing all the computers, servers and the HPC, also after we moved to the Máxima. Thanks also to the Hubrecht Institute for allowing us to stay the third floor of the new building when the Princess Máxima Center building was being built. Thanks for sharing your facilities with us, such as the bioanalyzer of the van Oudenaarden group or the microscopes of the Kops group. A big thank you to the media kitchen of the Hubrecht for making medium and sterilizing Erlenmeyers for us! In our own Máxima building we have the great help of Eugenie, thank you for making sure that our glassware is clean and the tip boxes are always filled and sterilized. A big thank you to Sabine and Martine as well for administrative support and help whenever we needed to send out packages. Thank you also to the Máxima research IT-department, especially Myriam, Lana and Reindert for help whenever our computers were misbehaving again.

When we just moved to the Máxima, our center was still small and we shared office spaces with some of the staff. Thanks Sander, Alieke, Nathan, Arman and others for sharing fun times, coffee chats and even joining some of the pub quizzes! I also want to thank my office-buddies in the Hubrecht-office: Bianca, Emmy, Saman, Philip, Sepide and Jules for being nice colleagues and a nice mix of people from different groups, and for hosting legendary Christmas borrels with virtual fire places and Glühwein from the water bath (set to $70^{\circ} \mathrm{C}$ ).

When we were still located at the Hubrecht the Máxima hosted a beach volleyball tournament, which we took way too seriously and we "smashed" all our opponents. Yes, we had great fun. Thanks for winning together Saman, Thomas and Juli!

I also would like to thank everyone from the board game gang, for great evenings filled with games, BBQ's, beers and a lot of fun. Thanks Wouter for joining even when your daughter was claiming most of your time, Bas for always being in for some fun and an extra beer, Margit for joining although you secretly don't really enjoy games that much, Caro for all the "potje kaarten in de bus" and Annabel for being amazing and having inherited awesome cooking skills through your Italian genes.

One of the moments to look forward to once every two weeks was the Florin pubquiz, as well as the pre-quiz burgers. Even though our team had many names (I still think the Worst-Kaas Scenario was the best) and many different people joined over the years (ranging from once to every two weeks), it was always a lot of fun. Thanks Bas (team captain!), Susan and Corina (for actually starting the tradition), Tim (and your knowledge about weird and obscure music), Alex, Margit, Saman, Marc, Javi 
(sports and Star Wars questions), Annabel, Lars, Philip (knowing everything from Wikipedia is useful during quizzes), Irene, Marit (listening to $3 \mathrm{fm}$ and knowing all the lyrics), Jeff (our team always needs to have an American), Kim (for being even more enthusiastic about the quiz than Bas and me), Reinier, Wouter, Eline, Freek, Gabi, Axel, Jurrian, Winnie, Eduard, Natanja, Janna, Michelle and all others that joined, for making Wednesday evening an evening to look forward to!

Thanks also to the futsal people! Chasing a ball with 12 people is a great way to relax and get energized in the middle of the week. Thanks especially to PJ, Javi and Axel Beier, for organizing the matches when I first joined. Only when I started organizing the futsal matches together with Saman and later with Axel Rosendahl-Huber I realized that this takes more time and effort than most people think. Thanks Saman and Axel Rosendahl-Huber for sharing the burden with me. Thanks to all the people that regularly or occasionally joined our Tuesday/Wednesday afternoon futsal matches. Thanks Guy, Enric, Alex, Joppe, Vinicius, Maartje, Eelco, Mauro, Geert, Nico, Ismaël, Sander, Wouter, Frank, Juli, Lucas, Marwan, Arman, Samu, Sonja, Pavol, Jens, Waleed, Cayetano, Lars, Erik, Manuel, Margit, Bas, Freek, Pieke, Jaap, Vlad, Ajit, Stijn, Winnie, Jan, Muhammad, Louk, Jooske, Mike, Sanne, Michael, Nader, Niels, Mark, Andrea and many others.

I would like to thank all the people that joined on our yearly and awesome trips to the Ardennes. Drinking a "few" beers together is a great way to start friendships or collaborations. A great big thank you for the organizers of the Ardennes weekends during the years that made this possible: Javi, Axel and Erik (yes, you are the wisest of them all)! Also thanks to my fellow quiz organizers, Bas and Wouter and of course our lovely assistant Charlotte, for organizing some great quizzes (never again in the pool though). Thank you Lennart, Ajit and Kim for cooking amazing meals and making sure we wouldn't starve during the weekends. Due to some strange coincidence the Ardennes people largely overlap with the people that frequent the borrels. As most people know I am a big fan of the borrels, since they help connect with people from other labs or institutes that you otherwise wouldn't really talk to. So in addition to all the Ardennes people I would like to thank the people that joined the borrels at the UMC Utrecht, the Hubrecht Institute as well as in the Princess Máxima Center. Thanks (in no particular order): Maria, Maaike, Marten, WJ, Koen, Lucas, Ingrid, Jasmin, Livio, Spiros, Bas Molenaar, Charlotte, Banafsheh, Annabel, Caro, Arianna, Lorenzo, Euclides, Ilia, Tim, Rob, Kim, Judith, Lotte, Laura, Colinda, Jessica, Geert, PJ, Maartje, Chloé, Corina, Alex, Mauro, Roxanne, Ramada, Juliëtte, Naomi, Margit, Lars, Rijndert, Ravian, Hannah, Lianne, Fabienne, Eline, Freek, Axel, Jurrian, Winnie, Eduard, Natanja, Janna, Michelle, Robin, Ryan, Camilla, Irene, Francisco, Evelyn, Sjors, Karlijn, Karin, Željko, Lindy Vernooi, Lindy Alles, Frank, Ruben, Marc, Jarno, Valentina, Emmy, Loes, Sanne, Rico, Claudia, Kaylee, Thomas, Nil, Celina, Britt, Sofie, Marlinde, Jordy and (believe it or not) many others for legendary Ardennes trips, for making the borrels fun, for sharing beers or for just nice random chats over the past six years.

Esther en Nienke, we trokken altijd samen op tijdens onze Bachelor en Master en 
daarna zijn we tegelijk (op andere plekken) aan onze PhD's begonnen. Jullie PhD's hadden helaas veel tegenslagen, maar gelukkig hebben jullie allebei een andere focus gevonden voor jullie passies. Bedankt voor de toffe (studenten) tijd samen en ook de gezellige tijd buiten de collegebanken. Zonder jullie was ik waarschijnlijk niet aan deze PhD begonnen

Naast alle wetenschappers en mensen uit Utrecht moet ik natuurlijk ook mijn andere vrienden bedanken. Allereerst mijn oude middelbare schoolvrienden met wie we ieder jaar het NKF (Nieuwjaars Kroketten Festijn) vieren. Ook al zijn we ruim 12 jaar geleden geslaagd van de middelbare school, nog steeds komen we steevast ieder jaar op 2 januari samen om de hele avond broodjes kroket te eten. Een geweldige traditie en hij staat alvast in mijn agenda voor de komende 12 jaar. Bedankt voor alle gezelligheid die we na al die jaren nog steeds hebben. Bedankt Andy, Paul, Jan, Matthijs, Jurre, Guido, Eric, Aldo, Robin, Joris, Thomas van der Wielen, Thomas Sterks, Mark, Kaj en Pepijn. Daarnaast moet ik natuurlijk Roel extra bedanken voor het hosten van het NKF. Ieder jaar weer bij je ouders, ook al woonde je er al lang niet meer. En sinds dit jaar in je eigen huis! Ik ben benieuwd hoe het er volgend jaar uitziet na de verbouwing. Aldo, ik denk nog steeds af en toe aan de filosofie lessen die we samen hadden en onze absurde hersenkronkels (ik weet niet meer waarom, maar we wisten zeker dat de aarde eigenlijk 2D is). Het is fijn om elkaar beschonken in de stad, bij festivals of tijdens carnaval tegen te komen. Robin (Robiiiiiin), het duurde even voordat we realiseerden dat als we in dezelfde stad wonen we elkaar ook best vaker dan eens per jaar kunnen zien. Onze avondjes Roti eten (50\% successrate) en schreeuwend op de bank Overcooked spelen waren altijd geweldig. Ik voel me ook ontzettend vereerd dat ik de eerste ben die je belt als je carnaval gaat vieren. Ik blijf bij wat ik gezegd heb, er zijn weinig mensen met wie ik zo veel en zo hard lach als met jou. Ik hoop dat we deze avondjes snel weer oppakken.

Scouting is altijd mijn hobby geweest, en was altijd een goede reden om naar het zonnige zuiden af te reizen om mijn zaterdagavonden (soms ook zondagochtenden) te vullen. De vele kampvuren, pionier projecten, wandelingen, bierproeverijen, dansprogramma's, casinoavonden, kookprogramma's en vele andere activiteiten, maar ook de biertjes later op de avond, beerpong en dansen tot diep in de nacht waren altijd een fijne afleiding van het werk. Dankjewel alle (ex-) pivo's: Koen, Ward, Björn, Maartje, Manon, Sam, Jeroen, Chris, Laura, Geerte, Lucas, Lina, Aafke, Elze, Emiel, Anne, Iris, Dik, Janneke, Tom, Joost, Renke en Aniek voor onze vriendschap binnen scouting, maar ook daarbuiten tijdens carnaval, Jazz in Duketown, Paaspop en alle andere evenementen. Aangezien ik geen pivo meer ben, maar ondertussen alweer even bij de stampij zit, wil ik uiteraard ook al mijn stampij-genoten bedanken voor net wat uitdagendere programma's en ook heel veel gezelligheid. Naast oud pivo-genoten die nu ook stampij lid zijn, bedankt ook Fred, Christien, Aukje, Hans, Gemma, Muriëlle, Martijn, Marijn, Camiel, Marco, Lenard, Janneke, Mariette, René, Femke, Frans, Roel, Noëlle, Jet, Floor en Jacqueline.

Niels en $\mathbf{Q}$, of eigenlijk Symb en CL. Thanks voor alle vrijdagavond gameavonden, gevuld met broodjes kroket, films, wandelingen, bier, cafeïne maar vooral 
gezelligheid en afleiding. Hoewel ik degene was die het vaakst afhaakte is onze vrijdagavond een belangrijke "cornerstone" van mijn week. Eén van de weinige tradities die ons studenten- en werkleven overleefd heeft en daar ben ik heel dankbaar om. Daarnaast ook de enige die de corona- (en thesis schrijf)tijd wist te doorstaan.

Het is gek hoe therapeutisch het kan zijn een hele avond een groot gat te maken om een enorme onderwatertuin te bouwen, om die vervolgens nooit te gebruiken. De hoeveelheid "geraide instances", gebouwde torens, geslachte zombies, "gepurgede" planeten zijn niet te tellen, behalve dat het gelijk staat aan vele, vele uren plezier. Ik hoop dat het een traditie is die we lang vol kunnen houden. Thanks also to the people that joined our game evenings, I am a great fan of "the more the merrier", so thank you Peter, Sander, Juriy and Jay! En natuurlijk heel veel dank aan Marjo en Wilbert voor de gastvrijheid en de vele maaltijden die we gehad hebben.

Natuurlijk wil ik iedereen bedanken van onze "vakantie gang", met wie we meerdere zomervakanties, escape rooms en vele avonden vol bier en plezier hebben doorgebracht. Half Europa hebben we door gecrost, inclusief de nodige autopech en de bijna-naar-Riga vakantie. Super bedankt voor de mooie tijden! Thanks Garben voor een mooie eerste trip binnen het eigen land. Thanks Dirk, voor onze eerste vakanties en roadtrips en humor waarmee we met een gestolen accu konden dealen. Thanks Ward voor het "invallen" en de trips naar Slowakije, Hongarije en Oekraïne. Thanks Stefan, voor alle vakanties (zonder elkaar aan te raken in bed) en discussies over rechtspraak en politiek. Bas, ook al ben jij nooit mee op vakantie geweest voelt het alsof we elkaar al heel lang kennen. Mijn voorstel is dat we dat we binnenkort een keer met jou gaan reizen. En als laatste persoon die er alle vakanties bij was en centraal stond voor al deze vriendschappen: Youri. Wat kan ik zeggen. In onze vriendschap heeft één ding altijd centraal gestaan, maar onze vriendschap is al heel lang veel meer dan alleen dat. (Oke fair enough, twee dingen dan. Onze variant van "Mac and Cheese" verdient een eervolle vermelding). Jij bent altijd een steun geweest, zowel tijdens mijn studententijd als tijdens mijn PhD. Ondanks dat we lang op verschillende plekken hebben gewoond, wisten we toch bijna altijd eens in de 1-2 weken af te spreken. Thanks voor alle potjes HALO die we samen gespeeld hebben, onze vakanties samen, spelletjes avonden, stap avonden (nooit de pot aan Youri geven) of de avondjes gewoon lekker bier drinken en slap ouwehoeren. Ik weet zeker dat, ondanks waar ik in de toekomst kom te wonen, we ons best blijven doen elkaar veel te zien! Dankjewel ook Kim, dat jij altijd mijn aanwezigheid en ons lawaai/gescheld tolereert, maar ook voor de escaperoom-, bier- en spelletjesavonden met $z^{\prime} n$ allen.

Jochem! Buddy, bruv. We zijn al lang vrienden en bijna 4 jaar huis- / kamergenoten geweest. Ik denk dat ik het met niemand anders op zo'n klein oppervlakte zo lang had volgehouden. De eerste helft van mijn PhD woonden we samen en dat was heerlijk. Je was vaak 's avonds weg maar als je er was konden we altijd samen eten en chillen (of niet, dat was ook oké). Samen spontaan naar de film of op het balkon biertjes drinken en over het leven praten was altijd geweldig. Je was de beste roommate die iemand zich kon wensen! Thanks voor alle levensadviezen 
en gewoon een geweldige vriend zijn. Ook dank voor jouw theater-vrienden die geregeld langskwamen, waarmee we gezellig avonden hebben gehad, bedankt Sophie (margaritas!), Kaylie, Sharon en Suzie.

Natuurlijk kan ik niet Jochem noemen zonder ook Heleen en Anne-Claire te bedanken. Ook al zien we elkaar (veel te) weinig, is onze vriendschap altijd heel sterk gebleven. We kunnen altijd alles met elkaar delen, alle frustraties kwijt maar ook mooie momenten delen. Onze avonden zijn altijd volledig gevuld met praten, en dan nog hebben we niet genoeg gezegd. Deze avonden hebben me zeker door mijn PhD heen geholpen. Ik ben heel blij dat we met $z^{\prime} n$ vieren zulke goede vrienden zijn en dat zal zeker zo blijven. Dank ook aan jullie vriendjes Stijn, Tobias en Luc die er regelmatig bij zijn!

Dan wil ik natuurlijk mijn familie bedanken voor hun steun tijdens de afgelopen zes jaar. Als eerste Vitas, jij kwam aan het begin van mijn $\mathrm{PhD}$ in ons leven en bent sindsdien altijd een extra reden geweest om naar Den Bosch af te reizen. Je bent altijd bijzonder blij om mij te zien en dat gevoel is wederzijds. Als je kwispelend achter de poort staat wanneer ik binnen kom vergeet ik altijd al mijn zorgen.

Bedankt ook voor mijn "schoonfamilie", Els en Lina. Dankjewel dat ik altijd welkom ben en jullie mij een deel van de familie laten voelen. Bedankt voor alle Sinterklaas avondjes en kerstdiners en dat jullie langskwamen toen ik in het ziekenhuis lag. Dat was heel fijn. Bedankt ook voor de rest van de familie van Beers voor alle gezellige Sinterklaas- en kerstavonden en natuurlijk voor de familieweekenden. Ook al is de familie groot, ik voelde me vanaf het begin thuis in deze hele diverse familie. Bedankt Lia, Cor, José, Victor, Fried, Ellen, Gerard, Crista, Marja, Wil, Sjef, Kobus, Cees, Mathieu, Marri, Kees, Geert, Bette, Doris, Joske, Geurt, Neele, Huub, Roos en Willem dat jullie deze rare jongen meteen accepteerden als lid van de familie. En natuurlijk ook Lennard. Vanaf het eerste moment hadden we meteen een super goede klik, waarschijnlijk omdat we allebei net wat gekker zijn dan de rest van jullie familie. Thanks voor al onze (politieke) discussies, potjes (beach)volleybal en biertjes tijdens familie-events en daarbuiten! Dank ook voor de anderen van de "koude kant" die begrijpen hoe het is om in deze familie ondergedompeld te worden.

Lieve zusjes! Ook al hadden we vroeger wel eens ruzie (welke broers en zussen niet?), onze band is alleen maar beter geworden nadat we allemaal het huis uit waren. Jorine, het is grappig hoe we langzaam steeds meer dezelfde vrienden gekregen hebben. Hierdoor zagen we elkaar niet alleen in de Hunzestraat maar ook als we samen naar feestjes gingen, tijdens carnaval en andere festiviteiten in de buurt van Den Bosch. Nu je in (de buurt van) Utrecht werkt ook af en toe als je bij ons komt eten en spelletjes doen, laten we dat vooral blijven doen. Mariole, het was altijd heel fijn om samen een biertje te drinken, mijn hart te luchten of gewoon bij te kletsen, in Utrecht, Nijmegen of in de pauzes van een van de vele cabaretvoorstellingen waar we samen heen zijn gegaan. Bedankt dat jij en Finbar mij altijd uitnodigden tijdens de vierdaagse feesten en dat ik altijd kon blijven slapen. Ik heb goede herinneringen samen met Finbar genietend van de muziek van So I Watch You From Afar en 
Animals As Leaders. Nu Lenny er is, is alles anders natuurlijk, maar dat is alleen maar een extra reden om elkaar vaker op te zoeken!

Lieve vader en moeder, ik kan jullie niet genoeg bedanken voor jullie steun en liefde en het warme en veilige thuis dat jullie ons gegeven hebben. Jullie stimuleerden mij altijd om mijn interesses te volgen en te doen wat ik graag wilde doen. Jullie zijn altijd geïnteresseerd in wat ik nou precies doe, ook al kunnen jullie er nooit echt iets van begrijpen. Bedankt dat jullie altijd voor me klaar staan en altijd naar Utrecht kwamen rijden als ik hulp nodig had. Jullie hadden er altijd begrip voor als ik te druk was om langs te komen. Het hielp enorm dat ondanks dat we elkaar minder zagen, jullie mij via de post iedere week een steuntje in de rug gaven. Ik had me geen betere ouders kunnen wensen.

Geerte, het is eigenlijk niet in woorden te omschrijven wat ik voor je voel. Dankjewel voor je onvoorwaardelijke liefde en steun tijdens de afgelopen jaren en voor je begrip wanneer (lab)werk weer eens de prioriteit had. Ik weet niet hoe ik het allemaal af had kunnen krijgen zonder je. Samen met jou ben ik op mijn gelukkigst en wat toekomst ook brengt, ik weet zeker dat het geweldig wordt omdat we samen zijn. Ik heb het al heel vaak tegen je gezegd, en zal dat zeker blijven doen: ik hou van je schatje. 


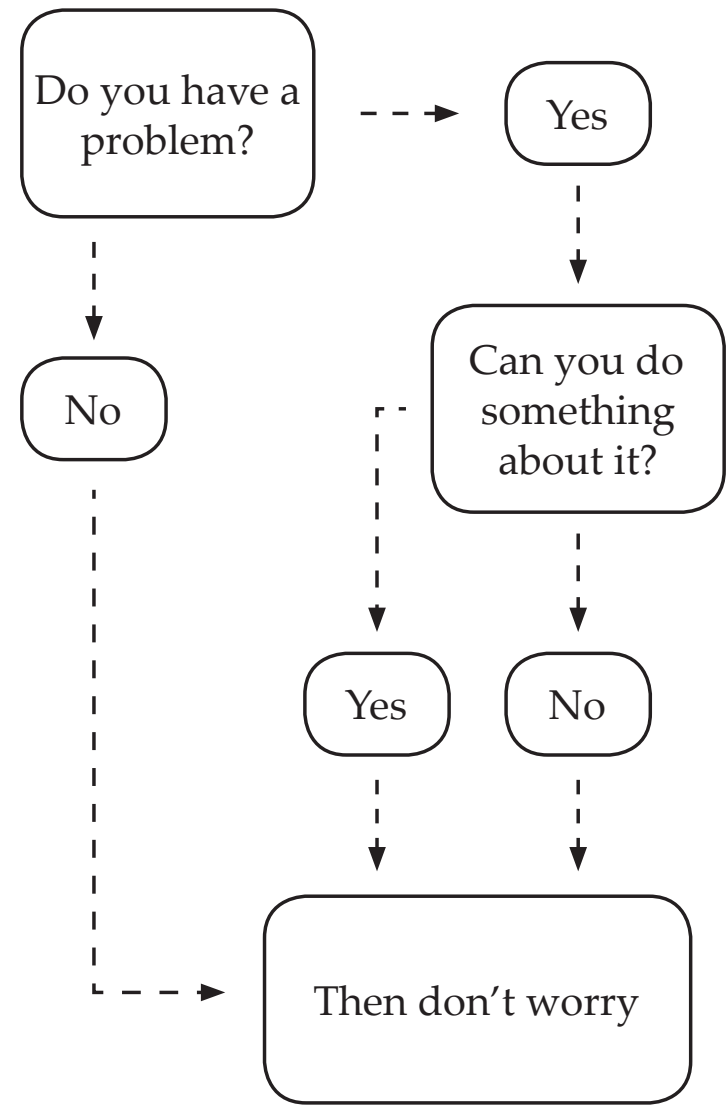



